\title{
Cooperative Bond Activation by a Bimetallic
}

\section{Main-Group Complex}

Oleksandr Kysliak, ${ }^{a}$ Helmar Görls, ${ }^{a}$ and Robert Kretschmera,b*

a) Dr. O. Kysliak, Dr. H. Görls, Prof. Dr. R. Kretschmer, Institute of Inorganic and Analytical Chemistry (IAAC), Friedrich Schiller University Jena, Humboldtstraße 8, 07743 Jena, Germany

b) Prof. Dr. R. Kretschmer, Jena Center for Soft Matter (JCSM), Friedrich Schiller University Jena, Philosophenweg 7, 07743 Jena, Germany

eMail: robert.kretschmer@uni-jena.de 


\section{Supporting Information}

Content

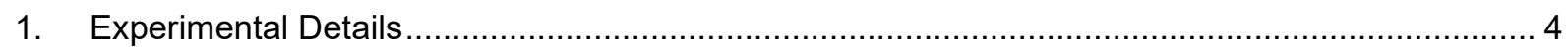

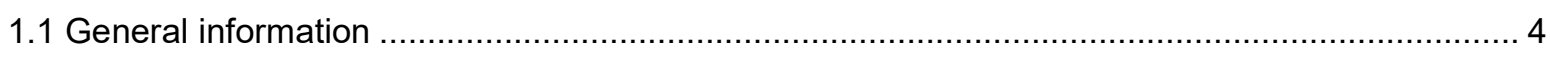

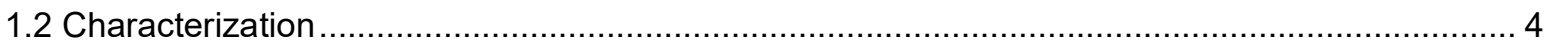

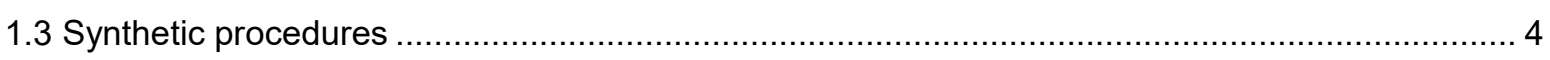

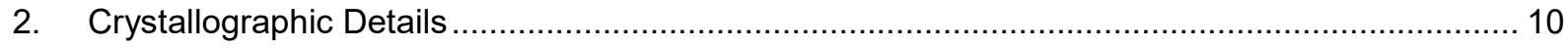

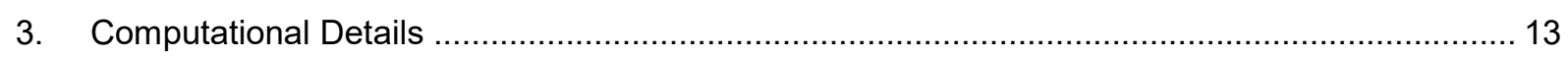

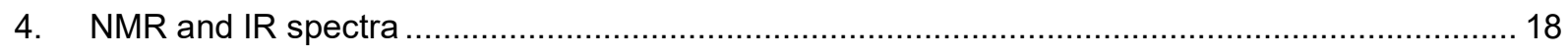

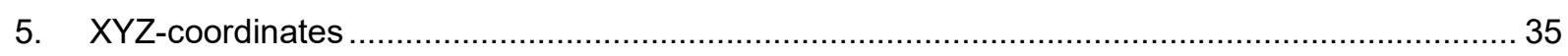

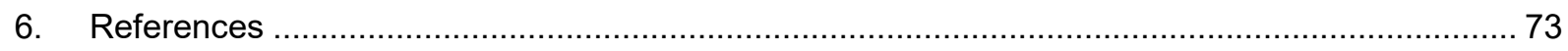




\section{Experimental Details}

\subsection{General information}

All preparations were performed under an inert atmosphere of dinitrogen by means of standard Schlenkline and glovebox (GS Systemtechnik and MBraun) techniques. All solvents (toluene, hexane) were distilled from $\mathrm{Na} /$ benzophenone prior to use while $\mathrm{C}_{6} \mathrm{D}_{6}$ and toluene- $\mathrm{d}_{8}$ were dried using molecular sieves. $\mathrm{KN}\left(\mathrm{SiMe}_{3}\right)_{2}\left(95 \%\right.$ purity), $\mathrm{C}_{6} \mathrm{~F}_{6}, \mathrm{C}_{6} \mathrm{~F}_{5} \mathrm{H}$, and $\mathrm{C}_{6} \mathrm{~F}_{4} \mathrm{H}_{2}$ were commercially available and used as received. The protio-ligand 1, ${ }^{\mathrm{S} 1}$ gallium(I) $\beta$-diketiminate $3,{ }^{\mathrm{S} 2}$ and $\mathrm{GaCp}^{*}$ (orange oil with density $1.2 \mathrm{~g}$ $\mathrm{mL}^{-1}$ and approx. $85 \%$ purity) $)^{\mathrm{S3}}$ have been synthesized according to literature procedures.

\subsection{Characterization}

The NMR spectra were recorded with Bruker Avance 400, Bruker Avance 600 and Bruker Fourier 300 spectrometers with $\delta$ referenced to external tetramethylsilane standard $\left({ }^{1} \mathrm{H}\right.$ and $\left.{ }^{13} \mathrm{C}\right) .{ }^{1} \mathrm{H}$ and ${ }^{13} \mathrm{C}$ NMR spectra were calibrated by using the solvent residual peak $\left(\mathrm{C}_{6} \mathrm{D}_{5} \mathrm{H}: \delta\left({ }^{1} \mathrm{H}\right)=7.16 \mathrm{ppm}, \mathrm{C}_{6} \mathrm{D}_{5} \mathrm{CD}_{2} \mathrm{H}: \delta\left({ }^{1} \mathrm{H}\right)\right.$ $=2.09 \mathrm{ppm})$ and the solvent peak $\left(\mathrm{C}_{6} \mathrm{D}_{6}: \delta\left({ }^{13} \mathrm{C}\right)=128.06 \mathrm{ppm}\right)$, respectively. Elemental analysis was performed with a Vario EL Cube (Elementar Analysensysteme $\mathrm{GmbH}$ ). IR spectra (given in $\mathrm{cm}^{-1}$ ) were recorded with an Agilent Cary 630 FT-IR spectrometer using a diamond ATR unit.

\subsection{Synthetic procedures}

2: To a Schlenk vessel containing $1.50 \mathrm{~g}(2.50 \mathrm{mmol})$ of 1 and $1.05 \mathrm{~g}(5.00 \mathrm{mmol})$ of $\mathrm{KN}\left(\mathrm{SiMe}_{3}\right)_{2}, 15$ $\mathrm{mL}$ of toluene were added. The mixture was heated at $90^{\circ} \mathrm{C}$ on stirring for 2 hours which led to full transformation of 1 ligand according to ${ }^{1} \mathrm{H}$ NMR. All volatiles were then removed in vacuum on heating up to $60{ }^{\circ} \mathrm{C}$. The thus obtained potassium salt was used for the next step without further purification. It was dissolved in $20 \mathrm{~mL}$ of toluene, $1.1 \mathrm{~mL}(5.50 \mathrm{mmol})$ of $\mathrm{GaCp}^{*}$ were added and the mixture was stirred on heating at $80^{\circ} \mathrm{C}$ overnight, which led to full conversion of the precursor to the product according to ${ }^{1} \mathrm{H}$ NMR spectroscopy. The brownish precipitate of $\mathrm{KCp}{ }^{*}$ was filtered off and the reaction mixture was then almost completely dried leaving crystalline material and only a few drops of solvent. The product were washed with $n$-hexane, and the hexane washings were additionally stored at $-30^{\circ} \mathrm{C}$ which yielded all together $1.38 \mathrm{mg}(1.88 \mathrm{mmol}, 75 \%)$ of 2 as yellow crystals (NMR pure). 
${ }^{1} \mathrm{H}$ NMR $\left(400 \mathrm{MHz}, \mathrm{C}_{6} \mathrm{D}_{6}\right): \delta=7.15-7.25 \mathrm{ppm}$ (m, aromatic protons of Dipp groups and residual solvent peaks), $5.04 \mathrm{ppm}$ (br, 2H, cyclohexyl ring- $\mathrm{CH}$ ), $4.97 \mathrm{ppm}(\mathrm{s}, 2 \mathrm{H}$, methine $\mathrm{CH}$ ), $3.04 \mathrm{ppm}$ (two overlapping sept., $\left.{ }^{3} \mathrm{JHH}_{\mathrm{HH}}=7 \mathrm{~Hz}, 4 \mathrm{H}, \mathrm{CHMe} 2\right), 2.23 \mathrm{ppm}(\mathrm{s}, 6 \mathrm{H}, \mathrm{CMe}), 1.83 \mathrm{ppm}\left(\mathrm{br}, 2 \mathrm{H}\right.$, cyclohexyl ring- $\left.\mathrm{CH}_{2}\right), 1.66$ ppm (s, 6H, CMe), 1.55 ppm (br, 2H, cyclohexyl ring- $\left.\mathrm{CH}_{2}\right), 1.34 \mathrm{ppm}\left(\mathrm{d},{ }^{3} \mathrm{~J}_{\mathrm{HH}}=7 \mathrm{~Hz}, 6 \mathrm{H}, \mathrm{CHMe}_{2}\right), 1.26$ ppm (two overlapping d, $\left.{ }^{3} \mathrm{JHH}_{\mathrm{HH}}=7 \mathrm{~Hz}, 12 \mathrm{H}, \mathrm{CHMe} 2\right), 1.11 \mathrm{ppm}\left(\mathrm{d},{ }^{3} \mathrm{~J}_{\mathrm{HH}}=7 \mathrm{~Hz}, 6 \mathrm{H}, \mathrm{CHMe}\right.$ ); ${ }^{13} \mathrm{C}\left\{{ }^{1} \mathrm{H}\right\} \mathrm{NMR}$ (101 MHz, $\mathrm{C}_{6} \mathrm{D}_{6}$ ): $\delta$ = 164.5 ppm, 160.5 ppm, 144.3 ppm, 143.8 ppm, 143.3 ppm, 126.4 ppm, 123.9 ppm, 123.8 ppm, 100.8 ppm, 65.3 ppm, 39.2 ppm, 28.6 ppm, 28.4 ppm, 25.9 ppm, 25.6 ppm, 24.1 ppm, 23.6 ppm, 23.5 ppm; elemental analysis $\left(\mathrm{C}_{40} \mathrm{H}_{58} \mathrm{Ga}_{2} \mathrm{~N}_{4}\right)$ found (calcd): C 65.28 (65.42); H 7.86 (7.96); N 7.48 (7.63)

4a: To a Schlenk vessel containing $100 \mathrm{mg}(0.136 \mathrm{mmol})$ of 2 dissolved in $5 \mathrm{~mL}$ of toluene, $20 \mu \mathrm{L}(0.173$ $\mathrm{mmol}$ ) of hexafluorobenzene were added with a microsyringe and the reaction mixture was heated for 18 hours at $105^{\circ} \mathrm{C}$. The solution was concentrated and stored at $+5^{\circ} \mathrm{C}$, which afforded $20 \mathrm{mg}(0.021$ $\mathrm{mmol}, 16 \%$ yield) of $\mathbf{4 a}$ as slightly yellowish microcrystalline precipitate. Please note that in an NMR experiment, 2 is consumed within one hour of heating to $105^{\circ} \mathrm{C}$.

${ }^{1} \mathrm{H}$ NMR $\left(600 \mathrm{MHz}, \mathrm{C}_{6} \mathrm{D}_{6}\right): \delta=6.95-7.20 \mathrm{ppm}(\mathrm{m}$, aromatic protons of Dipp groups and residual solvent peaks), $4.73 \mathrm{ppm}(\mathrm{s}, 1 \mathrm{H}$, methine $\mathrm{CH}), 4.59 \mathrm{ppm}(\mathrm{s}, 1 \mathrm{H}$, methine $\mathrm{CH}), 4.29 \mathrm{ppm}(\mathrm{br}, 1 \mathrm{H}$, cyclohexyl ring- $\mathrm{CH}$ ), $3.63 \mathrm{ppm}\left(\mathrm{br}, 1 \mathrm{H}\right.$, cyclohexyl ring- $\mathrm{CH}$ ), $3.27 \mathrm{ppm}$ (two overlapping sept., ${ }^{3} \mathrm{~J}_{\mathrm{HH}}=6 \mathrm{~Hz}, 2 \mathrm{H}$, $\mathrm{CHMe}$ ), 2.97 ppm (sept., ${ }^{3} \mathrm{JHH}_{\mathrm{H}}=6 \mathrm{~Hz}, 1 \mathrm{H}, \mathrm{CHMe}$ ), $2.88 \mathrm{ppm}$ (sept., $\left.{ }^{3} \mathrm{JHH}_{\mathrm{HH}}=6 \mathrm{~Hz}, 1 \mathrm{H}, \mathrm{CHMe} 2\right), 0.55-$ $1.80 \mathrm{ppm}$ and $2.11 \mathrm{ppm}$ (series of $\mathrm{d}, \mathrm{s}$ and br signals, $\mathrm{CHMe}$, $\mathrm{CMe}$, cyclohexyl ring- $\mathrm{CH}_{2}$ ); ${ }^{13} \mathrm{C}\left\{{ }^{1} \mathrm{H}\right\} \mathrm{NMR}$ (151 MHz, $\left.\mathrm{C}_{6} \mathrm{D}_{6}\right): \delta=168.1 \mathrm{ppm}, 166.5 \mathrm{ppm}, 166.2 \mathrm{ppm}, 164.6 \mathrm{ppm}, 146.4 \mathrm{ppm}, 145.6 \mathrm{ppm}, 143.8 \mathrm{ppm}$, 143.3 ppm, 142.9 ppm, 139.8 ppm, 127.4 ppm, 127.1 ppm, 125.0 ppm, 124.7 ppm, 124.5 ppm, 124.0 ppm, 99.3 ppm, 97.1 ppm, 62.8 ppm, 62.2 ppm, 34.0 ppm, 32.3 ppm, 30.0 ppm, 28.6 ppm, 28.4 ppm, 27.6 ppm, 26.0 ppm, 25.3 ppm, 25.1 ppm, 25.0 ppm, 24.7 ppm, 24.6 ppm, 24.5 ppm, 24.4 ppm, 24.2 ppm, 23.7 ppm, 21.3 ppm; ${ }^{19} \mathrm{~F}$ NMR (377 MHz, $\left.\mathrm{C}_{6} \mathrm{D}_{6}\right): \delta=-118.5 \mathrm{ppm}(\mathrm{C} F),-120.8 \mathrm{ppm}(\mathrm{C} F),-155.1$ ppm (t, $\left.{ }^{3} J_{\mathrm{FF}}=19 \mathrm{~Hz}, \mathrm{p}-\mathrm{CF}\right),-160.7 \mathrm{ppm}(\mathrm{CF}),-163.2 \mathrm{ppm}(\mathrm{CF}),-166.1 \mathrm{ppm}(\mathrm{GaF})$; Elemental analysis $\left(\mathrm{C}_{46} \mathrm{H}_{58} \mathrm{~F}_{6} \mathrm{Ga}_{2} \mathrm{~N}_{4}\right)$ \% found (calcd): C 59.98 (60.03), H 6.22 (6.35), N 5.84 (6.09). 
4b: To a Schlenk vessel containing $100 \mathrm{mg}(0.136 \mathrm{mmol})$ of 2 dissolved in $5 \mathrm{~mL}$ of toluene, $50 \mu \mathrm{L}(0.449$ mmol) of pentafluorobenzene were added with a microsyringe and the reaction mixture was heated for 19 hours at $105^{\circ} \mathrm{C}$. The solution was concentrated and stored at $+5^{\circ} \mathrm{C}$, which afforded $21 \mathrm{mg}(0.023$ mmol, $17 \%$ yield) of $\mathbf{4 b}$ as slightly yellowish microcrystalline precipitate. Please note that in an NMR experiment, $\mathbf{2}$ is consumed within two hours of heating to $105^{\circ} \mathrm{C}$.

${ }^{1} \mathrm{H}$ NMR $\left(600 \mathrm{MHz}, \mathrm{C}_{6} \mathrm{D}_{6}\right): \delta=6.95-7.20 \mathrm{ppm}(\mathrm{m}$, aromatic protons of Dipp groups and residual solvent peaks), $6.41 \mathrm{ppm}\left(\mathrm{m}, 1 \mathrm{H}, \mathrm{C}_{6} \mathrm{HF}_{4}\right), 4.74 \mathrm{ppm}(\mathrm{s}, 1 \mathrm{H}$, methine $\mathrm{CH}), 4.59 \mathrm{ppm}(\mathrm{s}, 1 \mathrm{H}$, methine $\mathrm{CH}), 4.28$ ppm (br, $1 \mathrm{H}$, cyclohexyl ring- $\mathrm{CH}$ ), 3.63 ppm (br, 1H, cyclohexyl ring- $\mathrm{CH}$ ), 3.29 ppm (two overlapping sept., ${ }^{3} \mathrm{JHH}_{\mathrm{HH}}=7.5 \mathrm{~Hz}, 2 \mathrm{H}, \mathrm{CHMe}$ ), $3.06 \mathrm{ppm}$ (sept., ${ }^{3} \mathrm{JHH}_{\mathrm{HH}}=7.5 \mathrm{~Hz}, 1 \mathrm{H}, \mathrm{CHMe}$ ), $2.91 \mathrm{ppm}$ (sept., ${ }^{3} \mathrm{JHH}_{\mathrm{HH}}=$ $7.5 \mathrm{~Hz}, 1 \mathrm{H}, \mathrm{CHMe} 2$ ), 0.65-1.85 ppm and $2.12 \mathrm{ppm}$ (series of d, s and br signals, $\mathrm{CHMe}_{2}, \mathrm{CMe}$, cyclohexyl ring- $\left.\mathrm{CH}_{2}\right) ;{ }^{13} \mathrm{C}\left\{{ }^{1} \mathrm{H}\right\}$ NMR (151 MHz, $\left.\mathrm{C}_{6} \mathrm{D}_{6}\right): \delta=168.0$ ppm, $166.4 \mathrm{ppm}, 166.1 \mathrm{ppm}, 164.6 \mathrm{ppm}, 146.4$ ppm, 145.6 ppm, 143.8 ppm, 143.4 ppm, 143.0 ppm, 139.9 ppm, 127.3 ppm, 127.0 ppm, 125.0 ppm, 124.6 ppm, 124.5 ppm, 124.0 ppm, 106.2 ppm, 106.0 ppm, 105.9 ppm, 99.3 ppm, 97.1 ppm, 62.8 ppm, 62.3 ppm, 34.0 ppm, 32.3 ppm, 30.0 ppm, 28.6 ppm, 28.4 ppm, 27.6 ppm, 25.8 ppm, 25.4 ppm, 25.2 ppm, 25.1 ppm, 25.0 ppm, 24.7 ppm, 24.6 ppm, 24.5 ppm, 24.4 ppm, 24.3 ppm, 23.7 ppm, 21.3 ppm; ${ }^{19} \mathrm{~F}$ NMR (377 MHz, $\left.\mathrm{C}_{6} \mathrm{D}_{6}\right): \delta=-120.0$ ppm (CF), -122.4 ppm (CF), -138.6 ppm (CF), -140.9 ppm (CF), -165.8 ppm ( $\mathrm{GaF})$; Elemental analysis $\left(\mathrm{C}_{46} \mathrm{H}_{59} \mathrm{~F}_{5} \mathrm{Ga}_{2} \mathrm{~N}_{4}\right) \%$ found (calcd): C 60.22 (61.22), H 6.41 (6.59), N 5.91 (6.21).

4c: To a Schlenk vessel containing $100 \mathrm{mg}(0.136 \mathrm{mmol})$ of 2 dissolved in $5 \mathrm{~mL}$ of toluene, $50 \mu \mathrm{L}(0.467$ mmol) of 1,2,3,4-tetrafluorobenzene were added with a microsyringe and the reaction mixture was heated for five days at $105^{\circ} \mathrm{C}$. The solution was concentrated and stored at $+5{ }^{\circ} \mathrm{C}$, which afforded 19 $\mathrm{mg}(0.021 \mathrm{mmol}, 16 \%$ yield $)$ of $4 \mathrm{c}$ as slightly yellowish microcrystalline precipitate. Please note that in an NMR experiment, 2 is consumed within 48 hours of heating to $105^{\circ} \mathrm{C}$.

${ }^{1} \mathrm{H}$ NMR $\left(600 \mathrm{MHz}, \mathrm{C}_{6} \mathrm{D}_{6}\right): \delta=6.95-7.20 \mathrm{ppm}(\mathrm{m}$, aromatic protons of Dipp groups and residual solvent peaks), 6.15-6.60 ppm (br signal and m, 2H, $\left.\mathrm{C}_{6} \mathrm{H}_{2} \mathrm{~F}_{3}\right), 4.75 \mathrm{ppm}(\mathrm{s}, 1 \mathrm{H}$, methine $\mathrm{CH}$ ), $4.60 \mathrm{ppm}(\mathrm{s}, 1 \mathrm{H}$, methine $\mathrm{CH}$ ), $4.30 \mathrm{ppm}(\mathrm{br}, 1 \mathrm{H}$, cyclohexyl ring- $\mathrm{CH}), 3.64 \mathrm{ppm}(\mathrm{br}, 1 \mathrm{H}$, cyclohexyl ring- $\mathrm{CH}), 3.34 \mathrm{ppm}$ (two overlapping sept., ${ }^{3} \mathrm{JHH}_{\mathrm{HH}}=6 \mathrm{~Hz}, 2 \mathrm{H}, \mathrm{CHMe}$ ), $3.15 \mathrm{ppm}$ (sept., ${ }^{3} \mathrm{JHH}_{\mathrm{HH}}=6 \mathrm{~Hz}, 1 \mathrm{H}, \mathrm{CHMe}$ ), $2.95 \mathrm{ppm}$ (sept., ${ }^{3} \mathrm{HHH}_{\mathrm{HH}}=6 \mathrm{~Hz}, 1 \mathrm{H}, \mathrm{CHMe}$ ), 0.65-1.85 ppm and $2.12 \mathrm{ppm}$ (series of $\mathrm{d}$, s and br signals, $\mathrm{CHMe}_{2}$, 
CMe, cyclohexyl ring- $\left.\mathrm{CH}_{2}\right) ;{ }^{13} \mathrm{C}\left\{{ }^{1} \mathrm{H}\right\} \mathrm{NMR}\left(151 \mathrm{MHz}, \mathrm{C}_{6} \mathrm{D}_{6}\right): \delta=166.3 \mathrm{ppm}, 165.8 \mathrm{ppm}, 164.4 \mathrm{ppm}, 146.6$ ppm, 144.0 ppm, 143.4 ppm, 143.3 ppm, 140.1 ppm, 125.0 ppm, 124.6 ppm, 124.4 ppm, 124.0 ppm, 99.2 ppm, 97.0 ppm, 62.7 ppm, 62.3 ppm, 34.0 ppm, 32.3 ppm, 32.0 ppm, 30.0 ppm, 28.6 ppm, 28.4 ppm, 27.7 ppm, 25.4 ppm, 25.2 ppm, 24.8 ppm, 24.7 ppm, 24.6 ppm, 24.5 ppm, 24.4 ppm, 23.8 ppm, 21.3 ppm, 14.4 ppm; ${ }^{19} \mathrm{~F}$ NMR (377 MHz, $\mathrm{C}_{6} \mathrm{D}_{6}$ ): $\delta=-95.0$ ppm (strong, CF), -97.7 ppm (weak, CF), 114.0 ppm (weak, CF), -116.5 ppm (strong, CF), -143.3 ppm (weak, CF), -145.3 ppm (strong, CF), $165.2 \mathrm{ppm}$ (strong, $\mathrm{GaF}$ ); Elemental analysis $\left(\mathrm{C}_{46} \mathrm{H}_{60} \mathrm{~F}_{4} \mathrm{Ga}_{2} \mathrm{~N}_{4}\right) \%$ found (calcd): $\mathrm{C} 61.43$ (62.47), H 6.87 (6.84), N 6.10 (6.33).

General remark for $\mathbf{5 a}$ and $\mathbf{5 b}$ : although the crude product did contain only one species besides small amounts of the starting material 3, upon work-up a minor second species could be evidenced by ${ }^{1} \mathrm{H}$ and ${ }^{19} \mathrm{~F}$ NMR spectroscopy in both cases. Notably, the ${ }^{1} \mathrm{H}$ NMR pattern is comparable to the main product and the resonances are only slightly shifted. A diffusion-ordered (DOSY) NMR experiment reveals a comparable mono-exponential diffusion behaviour for both ${ }^{1} \mathrm{H}$ resonances $(4.90$ and $4.93 \mathrm{ppm})$ of $\mathbf{5 b}$, which indicates a similar hydrodynamic radius. Finally, neither the ${ }^{1} \mathrm{H}$ NMR nor the IR spectrum of $5 \mathrm{a}$ or $\mathbf{5 b}$ give evidence for the presence of a $\mathrm{Ga}-\mathrm{H}$ or $\mathrm{Ga}-\mathrm{OH}$ group.

5a: a) NMR experiment: To a Young NMR tube containing $20 \mathrm{mg}(0.041 \mathrm{mmol})$ of 3 dissolved in $0.5 \mathrm{~mL}$ of toluene- $\mathrm{d}_{8}, 20 \mu \mathrm{L}(0.173 \mathrm{mmol})$ of hexafluorobenzene was added by microsyringe. The reaction mixture was heated for two days at $105{ }^{\circ} \mathrm{C}$, which led to an almost complete conversion of $\mathbf{3}$ according to ${ }^{1} \mathrm{H}$ NMR (Figure S29). The solution was transferred into a Schlenk vessel, concentrated and stored at $+5{ }^{\circ} \mathrm{C}$, which afforded small amount of colorless crystals of 5 a suitable for an XRD analysis and NMR measurements of the main product ( $90 \%$ purity by NMR - Figure S18).

b) Full scale synthesis: To a Schlenk vessel containing $100 \mathrm{mg}(0.205 \mathrm{mmol})$ of 3 dissolved in $5 \mathrm{~mL}$ of toluene, $100 \mu \mathrm{L}(0.898 \mathrm{mmol})$ of hexafluorobenzene were added with a microsyringe and the reaction mixture was heated for five days at $105^{\circ} \mathrm{C}$. The volatiles were removed in vacuum and the obtained oily residue was recrystallized from $n$-hexane, which afforded $42 \mathrm{mg}(0.062 \mathrm{mmol}, 30 \%$ yield $)$ of a mixture of $5 a$ and a side-product in a 4:1 ratio according to ${ }^{1} \mathrm{H}$ NMR (Figure S21) as colorless crystals.

Main product: ${ }^{1} \mathrm{H}$ NMR $\left(600 \mathrm{MHz}, \mathrm{C}_{6} \mathrm{D}_{6}\right): \delta=6.85-7.15 \mathrm{ppm}$ (m, aromatic protons of Dipp groups), 4.89 ppm (s, $1 \mathrm{H}$, methine $\mathrm{CH}$ ), 3.59 ppm (sept., ${ }^{3} \mathrm{~J}_{\mathrm{HH}}=6 \mathrm{~Hz}, 2 \mathrm{H}, \mathrm{CHMe}$ ), 2.98 ppm (sept., ${ }^{3} \mathrm{JHH}_{\mathrm{H}}=6 \mathrm{~Hz}, 2 \mathrm{H}$, $\left.\mathrm{CHMe}_{2}\right), 1.53 \mathrm{ppm}(\mathrm{s}, 6 \mathrm{H}, \mathrm{CMe}), 1.52 \mathrm{ppm}\left(\mathrm{d},{ }^{3} \mathrm{JHH}_{\mathrm{H}}=6 \mathrm{~Hz}, 6 \mathrm{H}, \mathrm{CHMe}\right), 1.16 \mathrm{ppm}\left(\mathrm{d},{ }^{3} \mathrm{JHH}_{\mathrm{HH}}=6 \mathrm{~Hz}, 6 \mathrm{H}\right.$, 
$\mathrm{CHMe}$ ), $1.02 \mathrm{ppm}\left(\mathrm{d},{ }^{3} \mathrm{JHH}_{\mathrm{HH}}=6 \mathrm{~Hz}, 6 \mathrm{H}, \mathrm{CHMe}\right.$ ), $0.62 \mathrm{ppm}\left(\mathrm{d},{ }^{3} \mathrm{~J}_{\mathrm{HH}}=6 \mathrm{~Hz}, 6 \mathrm{H}, \mathrm{CHMe}\right) ;{ }^{13} \mathrm{C}\left\{{ }^{1} \mathrm{H}\right\} \mathrm{NMR}$ (151 MHz, $\left.\mathrm{C}_{6} \mathrm{D}_{6}\right): \delta=170.5$ ppm, 144.8 ppm, 143.3 ppm, 138.8 ppm, 124.7 ppm, 124.0 ppm, 96.8 ppm, 28.4 ppm, 27.8 ppm, 24.5 ppm, 24.4 ppm, 24.2 ppm, 23.6 ppm, 23.1 ppm; $\left.{ }^{19} \mathrm{~F} \mathrm{NMR} \mathrm{(377} \mathrm{MHz,} \mathrm{C}_{6} \mathrm{D}_{6}\right)$ : $\delta=-119.1 \mathrm{ppm}(\mathrm{m}, \mathrm{CF}),-152.1 \mathrm{ppm}(\mathrm{m}, \mathrm{CF}),-161.4 \mathrm{ppm}(\mathrm{m}, \mathrm{CF}),-195.0 \mathrm{ppm}(\mathrm{br}, \mathrm{Ga} F)$.

Side product: ${ }^{1} \mathrm{H}$ NMR (600 MHz, $\left.\mathrm{C}_{6} \mathrm{D}_{6}\right): \delta=6.85-7.15 \mathrm{ppm}$ (m, aromatic protons of Dipp groups), 4.90 ppm (s, $1 \mathrm{H}$, methine $\mathrm{CH}$ ), $3.59 \mathrm{ppm}$ (overlapping with the signal of the main product, sept., ${ }^{3} \mathrm{HH}=6 \mathrm{~Hz}$, $2 \mathrm{H}, \mathrm{CHMe}$ ), $2.89 \mathrm{ppm}$ (sept., ${ }^{3} \mathrm{HHH}_{\mathrm{HH}}=6 \mathrm{~Hz}, 2 \mathrm{H}, \mathrm{CHMe}$ ), $1.58 \mathrm{ppm}\left(\mathrm{d},{ }^{3}{ }_{\mathrm{HH}}=6 \mathrm{~Hz}, 6 \mathrm{H}, \mathrm{CHMe}_{2}\right), 1.53$ ppm (overlapping with the signal of the main product, $\mathrm{s}, 6 \mathrm{H}, \mathrm{CMe}$ ), $1.16 \mathrm{ppm}$ (overlapping with the signal of the main product, $\mathrm{d},{ }^{3} \mathrm{~J}_{\mathrm{HH}}=6 \mathrm{~Hz}, 6 \mathrm{H}, \mathrm{CHMe}$ ), $1.01 \mathrm{ppm}$ (overlapping with the signal of the main product, $\left.\mathrm{d},{ }^{3} \mathrm{JHH}_{\mathrm{HH}}=6 \mathrm{~Hz}, 6 \mathrm{H}, \mathrm{CHMe}\right), 0.44 \mathrm{ppm}\left(\mathrm{d},{ }^{3} \mathrm{JHH}_{\mathrm{HH}}=6 \mathrm{~Hz}, 6 \mathrm{H}, \mathrm{CHMe} 2\right) ;{ }^{19} \mathrm{~F} \mathrm{NMR}\left(377 \mathrm{MHz}, \mathrm{C}_{6} \mathrm{D}_{6}\right)$ : $-121.1 \mathrm{ppm}(\mathrm{m}, \mathrm{CF}),-153.5 \mathrm{ppm}(\mathrm{m}, \mathrm{CF}$ ) (other peaks are supposedly overlapping).

Elemental analysis of the full-scale synthesis sample $\left(\mathrm{C}_{35} \mathrm{H}_{41} \mathrm{~F}_{6} \mathrm{GaN}_{2}\right) \%$ found (calcd): $\mathrm{C} 62.44$ (62.42), H 6.21 (6.14), N 4.18 (4.16).

5b: To a Young vessel containing $150 \mathrm{mg}(0.308 \mathrm{mmol})$ of 3 dissolved in $3 \mathrm{~mL}$ of toluene, $50 \mu \mathrm{L}(0.449$ $\mathrm{mmol}$ ) of pentafluorobenzene were added with a microsyringe and the reaction mixture was heated for 12 days at $105^{\circ} \mathrm{C}$, which led to an almost full consumption of 3 according to ${ }^{1} \mathrm{H}$ NMR. The solution was transferred to a Schlenk flask, the volatiles were removed in vacuum and the obtained oily residue was redissolved in $n$-hexane and stored at $+5{ }^{\circ} \mathrm{C}$, which afforded $31 \mathrm{mg}(0.047 \mathrm{mmol}, 15 \%$ yield $)$ of the mixture of $\mathbf{5 b}$ and a side-product in a 3:1 ratio according to ${ }^{1} \mathrm{H}$ NMR (Figure S22).

Main product: ${ }^{1} \mathrm{H}$ NMR (400 MHz, $\left.\mathrm{C}_{6} \mathrm{D}_{6}\right): \delta=6.90-7.15 \mathrm{ppm}$ (m, aromatic protons of Dipp groups), 6.31 ppm (m, $\left.1 \mathrm{H}, \mathrm{C}_{6} \mathrm{~F}_{4} H\right), 4.90$ ppm (s, $1 \mathrm{H}$, methine $\mathrm{CH}$ ), $3.60 \mathrm{ppm}$ (sept., ${ }^{3} \mathrm{~J}_{\mathrm{HH}}=7 \mathrm{~Hz}, 2 \mathrm{H}, \mathrm{CHMe}$ ), 3.05 ppm (sept., ${ }^{3} \mathrm{JHH}_{\mathrm{HH}}=7 \mathrm{~Hz}, 2 \mathrm{H}, \mathrm{CHMe}$ ), $1.55 \mathrm{ppm}\left(\mathrm{s}, 6 \mathrm{H}, \mathrm{CMe}\right.$ ), $1.53 \mathrm{ppm}\left(\mathrm{d},{ }^{3} \mathrm{JHH}_{\mathrm{HH}}=7 \mathrm{~Hz}, 6 \mathrm{H}, \mathrm{CHMe}\right.$ ), $1.16 \mathrm{ppm}\left(\mathrm{d},{ }^{3} \mathrm{JHH}_{\mathrm{HH}}=7 \mathrm{~Hz}, 6 \mathrm{H}, \mathrm{CHMe}\right), 1.05 \mathrm{ppm}\left(\mathrm{d},{ }^{3} \mathrm{~J}_{\mathrm{HH}}=7 \mathrm{~Hz}, 6 \mathrm{H}, \mathrm{CHMe}\right), 0.62 \mathrm{ppm}\left(\mathrm{d},{ }^{3} \mathrm{JHH}_{\mathrm{HH}}=7 \mathrm{~Hz}\right.$, $6 \mathrm{H}, \mathrm{CHMe} 2) ;{ }^{13} \mathrm{C}\left\{{ }^{1} \mathrm{H}\right\} \mathrm{NMR}\left(101 \mathrm{MHz}, \mathrm{C}_{6} \mathrm{D}_{6}\right): \delta=170.7 \mathrm{ppm}, 145.2 \mathrm{ppm}, 143.8 \mathrm{ppm}, 139.2 \mathrm{ppm}, 125.1$ ppm, 124.4 ppm, 107.6 ppm, 97.1 ppm, 28.8 ppm, 28.1 ppm, 25.0 ppm, 24.8 ppm, 24.6 ppm, 24.1 ppm, $23.5 \mathrm{ppm} ;{ }^{19} \mathrm{~F}$ NMR $\left(282 \mathrm{MHz}, \mathrm{C}_{6} \mathrm{D}_{6}\right): \delta=-121.0 \mathrm{ppm}(\mathrm{m}, \mathrm{CF}),-139.0 \mathrm{ppm}(\mathrm{m}, \mathrm{CF}),-196.0 \mathrm{ppm}(\mathrm{br}$, $\mathrm{GaF})$.

Side product: ${ }^{1} \mathrm{H}$ NMR (400 MHz, $\left.\mathrm{C}_{6} \mathrm{D}_{6}\right): \delta=6.90-7.15 \mathrm{ppm}$ (m, aromatic protons of Dipp groups), 6.44 ppm (m, 1H, $\left.\mathrm{C}_{6} \mathrm{~F}_{4} H\right)$, $4.93 \mathrm{ppm}(\mathrm{s}, 1 \mathrm{H}$, methine $\mathrm{CH}$ ), $3.60 \mathrm{ppm}$ (overlapping with the signal of the main 
product, sept., ${ }^{3} \mathrm{JHH}_{\mathrm{HH}}=7 \mathrm{~Hz}, 2 \mathrm{H}, \mathrm{CHMe}$ ), $2.99 \mathrm{ppm}$ (sept., ${ }^{3} \mathrm{JHH}_{\mathrm{HH}}=7 \mathrm{~Hz}, 2 \mathrm{H}, \mathrm{CHMe}$ ), $1.60 \mathrm{ppm}\left(\mathrm{d},{ }^{3} \mathrm{~J}_{\mathrm{HH}}=\right.$ $7 \mathrm{~Hz}, 6 \mathrm{H}, \mathrm{CHMe}$ ), $1.55 \mathrm{ppm}$ (overlapping with the signal of the main product, s, $6 \mathrm{H}, \mathrm{CMe}$ ), $1.18 \mathrm{ppm}$ (partially overlapping with the signal of the main product, $d,{ }^{3} J_{\mathrm{HH}}=7 \mathrm{~Hz}, 6 \mathrm{H}, \mathrm{CHMe}$ ), $1.05 \mathrm{ppm}$ (overlapping with the signal of the main product, $\mathrm{d},{ }^{3} \mathrm{~J}_{\mathrm{HH}}=7 \mathrm{~Hz}, 6 \mathrm{H}, \mathrm{CHMe}$ ), $0.49 \mathrm{ppm}\left(\mathrm{d},{ }^{3} \mathrm{JHH}_{\mathrm{HH}}=7 \mathrm{~Hz}\right.$, $\left.6 \mathrm{H}, \mathrm{CHMe}_{2}\right) .{ }^{19} \mathrm{~F} \mathrm{NMR}\left(282 \mathrm{MHz}, \mathrm{C}_{6} \mathrm{D}_{6}\right):-122.9 \mathrm{ppm}(\mathrm{m}, \mathrm{CF}$ ) (other peaks are supposedly overlapping). Elemental analysis of the full-scale synthesis sample $\left(\mathrm{C}_{35} \mathrm{H}_{42} \mathrm{~F}_{5} \mathrm{GaN}_{2}\right) \%$ found (calcd): $\mathrm{C} 64.06$ (64.14), H 6.66 (6.46), N 4.24 (4.27). 


\section{Crystallographic Details}

The intensity data for all compounds were collected on a Nonius KappaCCD diffractometer using graphite-monochromated Mo-K $\mathrm{K}_{\alpha}$ radiation. Data were corrected for Lorentz and polarization effects; absorption was taken into account on a semi-empirical basis using multiple-scans. ${ }^{[\mathrm{S4}-\mathrm{S6}]}$ The structures were solved by direct methods (SHELXT[S7]) and refined by full-matrix least squares techniques against $\mathrm{Fo}^{2}$ (SHELXL-2018[S8]). All hydrogen atoms were included at calculated positions with fixed thermal parameters. All non-hydrogen atoms were refined anisotropically.[58] In the related figures, hydrogen atoms except those of the fluoroarenes are omitted for the sake of clarity. Crystallographic data as well as structure solution and refinement details are summarized in Table S1. Olex2 was used for structure representations; ellipsoids are displayed at $50 \%$ probability. ${ }^{[S 9]}$

The gallium(II) complexes $\mathbf{4 a - c}$ crystalize in the centrosymmetric space groups $\mathrm{P} 2{ }_{1} / \mathrm{c}(\mathbf{4 a}, \mathbf{4 b})$ and $\mathrm{Pbca}$ (4c) and accommodate hence equal numbers of the R,R- and S,S-diastereomers.

Notably, $\mathbf{2}$ and $\mathbf{5 a}$ crystallize as two and three independent molecules, respectively, and the structural fitting is given in Figure $\mathrm{S} 1$ and $\mathrm{S} 2$.

Crystallographic data (excluding structure factors) have been deposited with the Cambridge Crystallographic Data Center as supplementary publication CCDC-2039630 for 2, CCDC-2039631 for 4a, CCDC-2039632 for 4b, CCDC-2039633 for 4c, and CCDC-2039634 for 5a. Copies of the data can be obtained free of charge on application to CCDC, 12 Union Road, Cambridge CB2 1EZ, UK [E- mail: deposit@ccdc.cam.ac.uk].

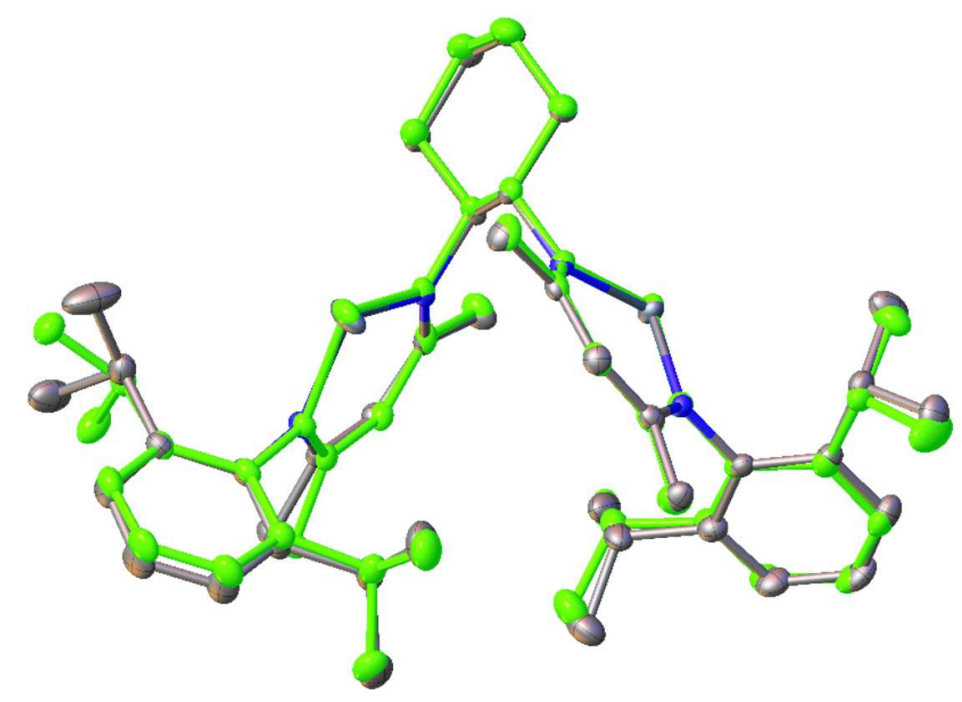

Figure S1. Fit of the two symmetry-independent molecules of 2 as observed by X-ray diffraction analysis. 
a)

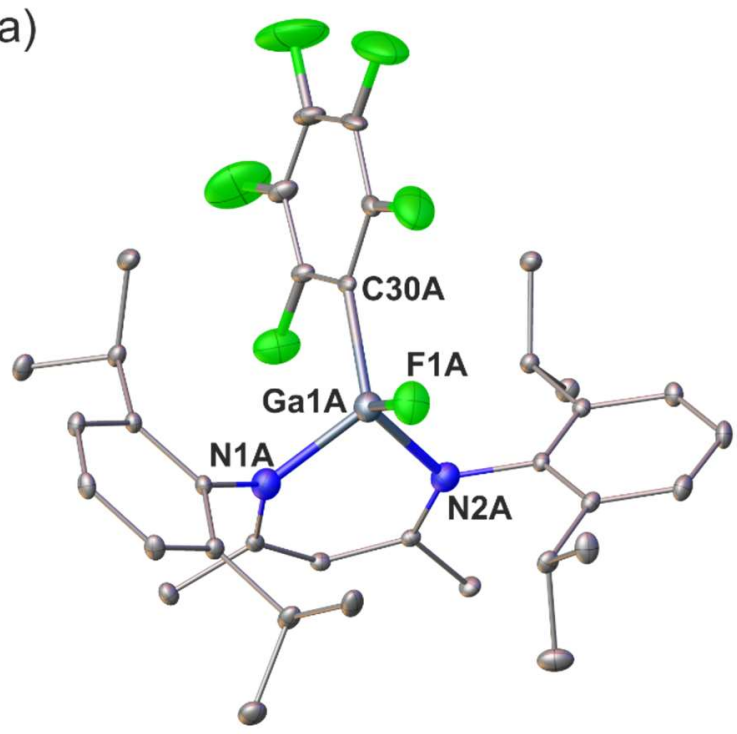

b)

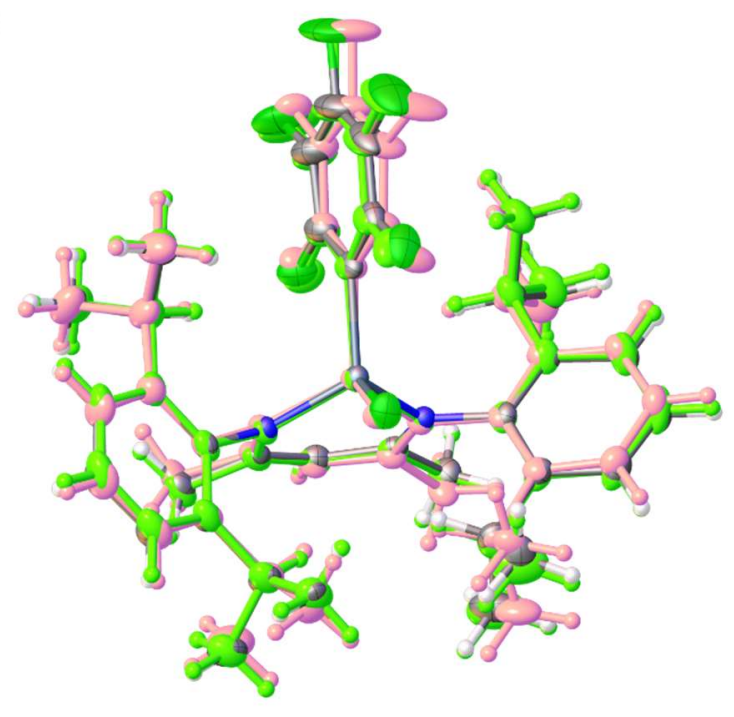

Figure S2. a) Solid-state structure of 5a (hydrogen atoms are omitted for the sake of clarity) with selected bond lengths $[\AA]$ ] and angles [ $\left.{ }^{\circ}\right]$ : Ga1A-N1A 1.9182(17), Ga1A-N2A 1.9312(17), Ga1A-F1A 1.7726(12), Ga1A-C30A 1.991(2), N1A-Ga1A-N2A 98.13(7). b) Fit of the three symmetry-independent molecules of $\mathbf{5 a}$ as observed by $\mathrm{X}$-ray diffraction analysis. 
Table S1. Crystal data and refinement details for the X-ray structure determinations.

\begin{tabular}{|c|c|c|c|c|c|}
\hline Compound & 2 & $4 a$ & $4 b$ & $4 c$ & $5 a$ \\
\hline formula & $\mathrm{C}_{40} \mathrm{H}_{58} \mathrm{Ga}_{2} \mathrm{~N}_{4}$ & $\mathrm{C}_{46} \mathrm{H}_{58} \mathrm{~F}_{6} \mathrm{Ga}_{2} \mathrm{~N}_{4}$ & $\mathrm{C}_{46} \mathrm{H}_{59} \mathrm{~F}_{5} \mathrm{Ga}_{2} \mathrm{~N}_{4}$ & $\mathrm{C}_{46} \mathrm{H}_{60} \mathrm{~F}_{4} \mathrm{Ga}_{2} \mathrm{~N}_{4}$ & $\mathrm{C}_{35} \mathrm{H}_{41} \mathrm{~F}_{6} \mathrm{GaN}_{2}$ \\
\hline $\mathrm{fw}\left(\mathrm{g} \cdot \mathrm{mol}^{-1}\right)$ & 734.34 & 920.40 & 902.41 & 884.42 & 673.42 \\
\hline $\mathrm{T} /{ }^{\circ} \mathrm{C}$ & $-140(2)$ & $-140(2)$ & $-140(2)$ & $-140(2)$ & $-140(2)$ \\
\hline crystal system & triclinic & monoclinic & monoclinic & orthorhombic & monoclinic \\
\hline space group & $P_{\bar{I}}$ & $\mathrm{P} 21 / \mathrm{C}$ & $\mathrm{P} 21 / \mathrm{C}$ & $\mathrm{Pbca}$ & $P 21 / c$ \\
\hline al $\AA$ & $15.2256(3)$ & $20.9909(6)$ & $20.894(4)$ & $16.0417(4)$ & $22.6354(2)$ \\
\hline$b / \AA$ & 15.9839(3) & $15.9277(4)$ & 15.910(3) & $13.1813(3)$ & $17.8525(2)$ \\
\hline$c / \AA$ & $16.4550(3)$ & $13.2611(3)$ & $13.262(3)$ & $40.9677(9)$ & $26.5186(3)$ \\
\hline$\alpha /^{\circ}$ & 89.795(1) & 90 & 90 & 90 & 90 \\
\hline$\beta /^{\circ}$ & 84.085(1) & $91.481(2)$ & $92.87(3)$ & 90 & $110.191(1)$ \\
\hline$y /^{\circ}$ & $76.873(1)$ & 90 & 90 & 90 & 90 \\
\hline$V / \AA^{3}$ & $3878.34(13)$ & $4432.2(2)$ & $4403.1(15)$ & $8662.6(3)$ & $10057.59(19)$ \\
\hline$Z$ & 4 & 4 & 4 & 8 & 12 \\
\hline$\rho\left(\mathrm{g} \cdot \mathrm{cm}^{-3}\right)$ & 1.258 & 1.379 & 1.361 & 1.356 & 1.334 \\
\hline$\mu\left(\mathrm{cm}^{-1}\right)$ & 14.21 & 12.77 & 12.81 & 12.98 & 8.8 \\
\hline measured data & 52426 & 59010 & 32792 & 48277 & 103144 \\
\hline data with $\mathrm{I}>2 \sigma(\mathrm{I})$ & 14486 & 7322 & 8023 & 7976 & 19040 \\
\hline unique data ( $\left.R_{\text {int }}\right)$ & $17683 / 0.0354$ & $10150 / 0.0809$ & $10080 / 0.0682$ & 9896/0.0638 & 23019/0.0296 \\
\hline $\left.\mathrm{w} R_{2}\left(\text { all data, on } \mathrm{F}^{2}\right)^{\mathrm{a}}\right)$ & 0.0933 & 0.0970 & 0.1203 & 0.1550 & 0.0902 \\
\hline$R_{1}(I>2 \sigma(I))^{\text {a })}$ & 0.0432 & 0.0487 & 0.0626 & 0.0544 & 0.0405 \\
\hline$S$ b) & 1.108 & 1.051 & 1.171 & 1.061 & 1.091 \\
\hline Res. dens./e. $\AA^{-3}$ & $0.424 /-0.442$ & $0.503 /-0.631$ & $1.122 /-0.994$ & $1.597 /-1.532$ & $1.177 /-1.249$ \\
\hline absorpt method & multi-scan & multi-scan & multi-scan & multi-scan & multi-scan \\
\hline absorpt corr $\mathrm{T}_{\min } / \max$ & $0.6470 / 0.7456$ & $0.6290 / 0.7457$ & $0.6972 / 0.7456$ & $0.4225 / 0.7456$ & $0.6721 / 0.7456$ \\
\hline CCDC No. & 2039630 & 2039631 & 2039632 & 2039633 & 2039634 \\
\hline
\end{tabular}

a) Definition of the $R$ indices: $R_{1}=\left(\Sigma|| F_{0}|-| F_{\mathrm{c}}||\right) / \Sigma\left|F_{\mathrm{o}}\right|$;

$\mathrm{wR}_{2}=\left\{\Sigma\left[w\left(F_{\mathrm{o}}^{2}-F_{\mathrm{c}}^{2}\right)^{2}\right] / \Sigma\left[w\left(F_{\mathrm{o}}^{2}\right)^{2}\right]\right\}^{1 / 2}$ with $w^{-1}=\square^{2}\left(F_{\mathrm{o}}^{2}\right)+(\mathrm{aP})^{2}+\mathrm{bP} ; \mathrm{P}=\left[2 \mathrm{~F}_{\mathrm{c}}^{2}+\operatorname{Max}\left(\mathrm{F}_{\mathrm{o}}^{2}\right)\right] / 3 ;$

b) $s=\left\{\Sigma\left[w\left(F_{\mathrm{o}}^{2}-F_{\mathrm{c}}^{2}\right)^{2}\right] /\left(N_{\mathrm{o}}-N_{\mathrm{p}}\right)\right\}^{1 / 2}$. 


\section{Computational Details}

All Geometry optimizations and frequency calculations were performed with the Gaussian 16 program package $^{\mathrm{S10}}$ in conjunction with the M06-2X hybrid functional ${ }^{\mathrm{S11}}$ and 6-31G(d) basis sets. ${ }^{\mathrm{S} 12}$ The absence of imaginary frequencies confirmed stationary points as minima, while optimized structures were identified as transition states when having only one imaginary frequency.

Single-point energies were calculated with ORCA 4.2.1 ${ }^{\mathrm{s} 13}$ using the domain-based local pair-natural orbital (DLPNO) approach to the $\operatorname{CCSD}(\mathrm{T})$ method, i.e., DLPNO-CCSD $(\mathrm{T}) ;$; 14 the default "NormalPNO" DLPNO settings were used. The triple- $\zeta c c-p V T Z$ basis set ${ }^{\mathrm{S} 15}$ was applied in conjunction with cc-pVTZ/C auxiliary basis sets. ${ }^{\mathrm{S} 16}$ For the calculation of Coulomb and exchange integrals in the HF reference, the RIJCOSX approximation ${ }^{S 17}$ using def2/J auxiliary basis sets ${ }^{S 18}$ was applied. In the $\operatorname{CCSD}(\mathrm{T})$ calculations the $\mathrm{T} 1$ diagnostics are lower than 0.02 , which gives evidence of an adequate description by this method. ${ }^{S 19}$ The largest doubles amplitudes of the DLPNO-CCSD(T) calculations were examined giving no indication of a multireference character.

The minimum energy paths (MEPs) were calculated at the M06-2X/6-31G(d) level of theory ${ }^{S 11, S 12}$ using the Nudged Elastic Band (NEB) method ${ }^{\mathrm{S} 20}$ implemented in ORCA 4.2.1. ${ }^{\mathrm{S} 13}$ 3D-structures of molecules were generated using CYLview and POVray. 21

In addition to Int1, Int1', which is best described as a fluoro-substituted 7,8-digallabicyclo[4.2.0]octa2,4-diene, and another encounter complexes (Int1"), which features weak $\mathrm{C}-\mathrm{H} \cdots \mathrm{F}-\mathrm{C}$ contacts as well as shortened Ga-Ga distances (2.66 to $2.68 \AA$ ), could be located on the potential-energy surfaces but both isomers are energetically less favored than Int1.

Notably, the transition structures TS1a and TS1c exhibit a small second imaginary frequency (-17.7 and $-10.8 \mathrm{~cm}^{-1}$, respectively) of marginal intensity ( 0.46 and 0.33 , respectively) in addition to the major imaginary frequency $\left(-291.3\right.$ and $-298.2 \mathrm{~cm}^{-1}$, respectively), i.e., being not a true first order saddle point. 


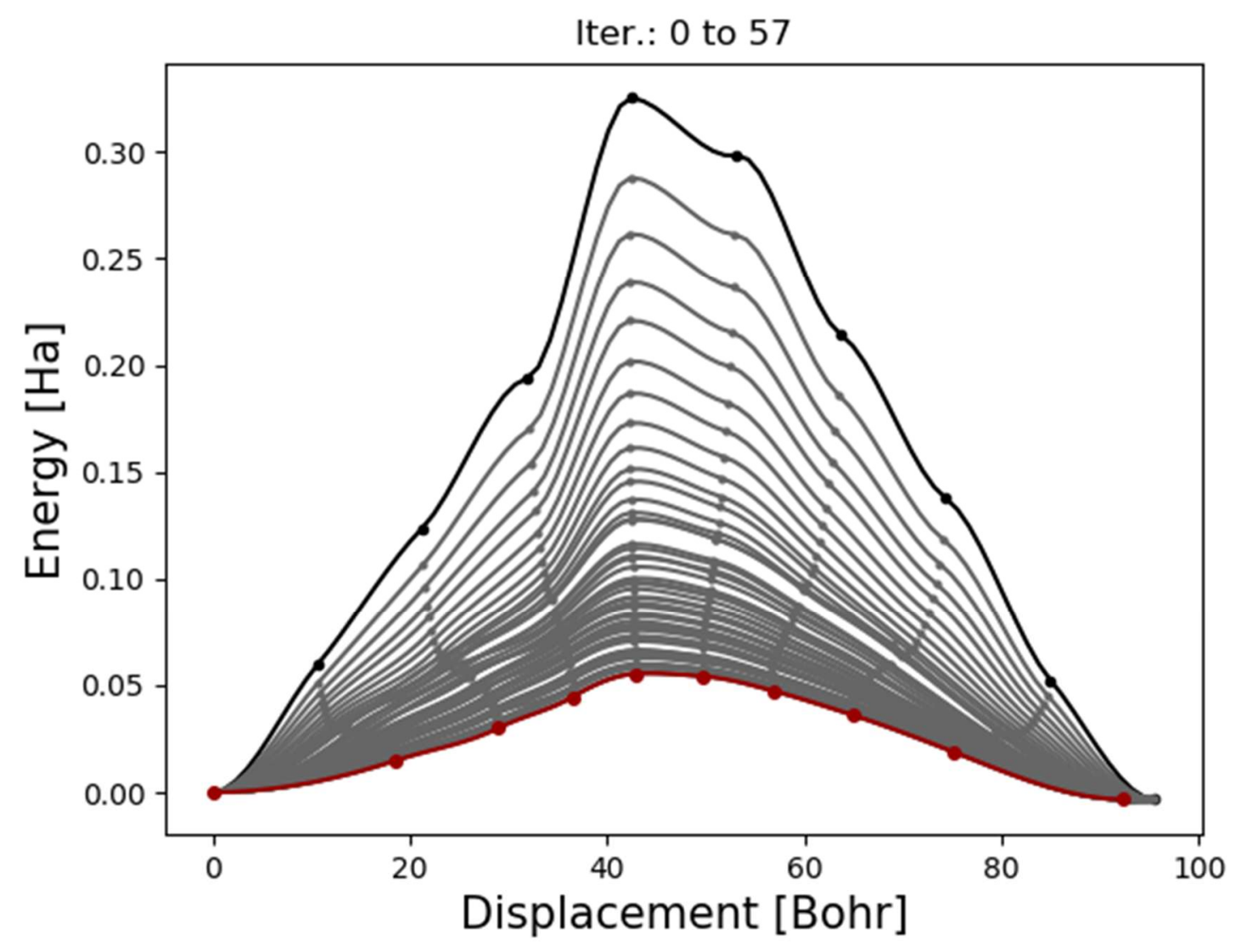

Figure S3. Minimum-energy path (MEP) in going from 2 (left) to 2' (right) using the nudged elastic band approach at the M06-2X/6-31G(d) level of theory. Notably, the uncorrected electronic energy is given. 


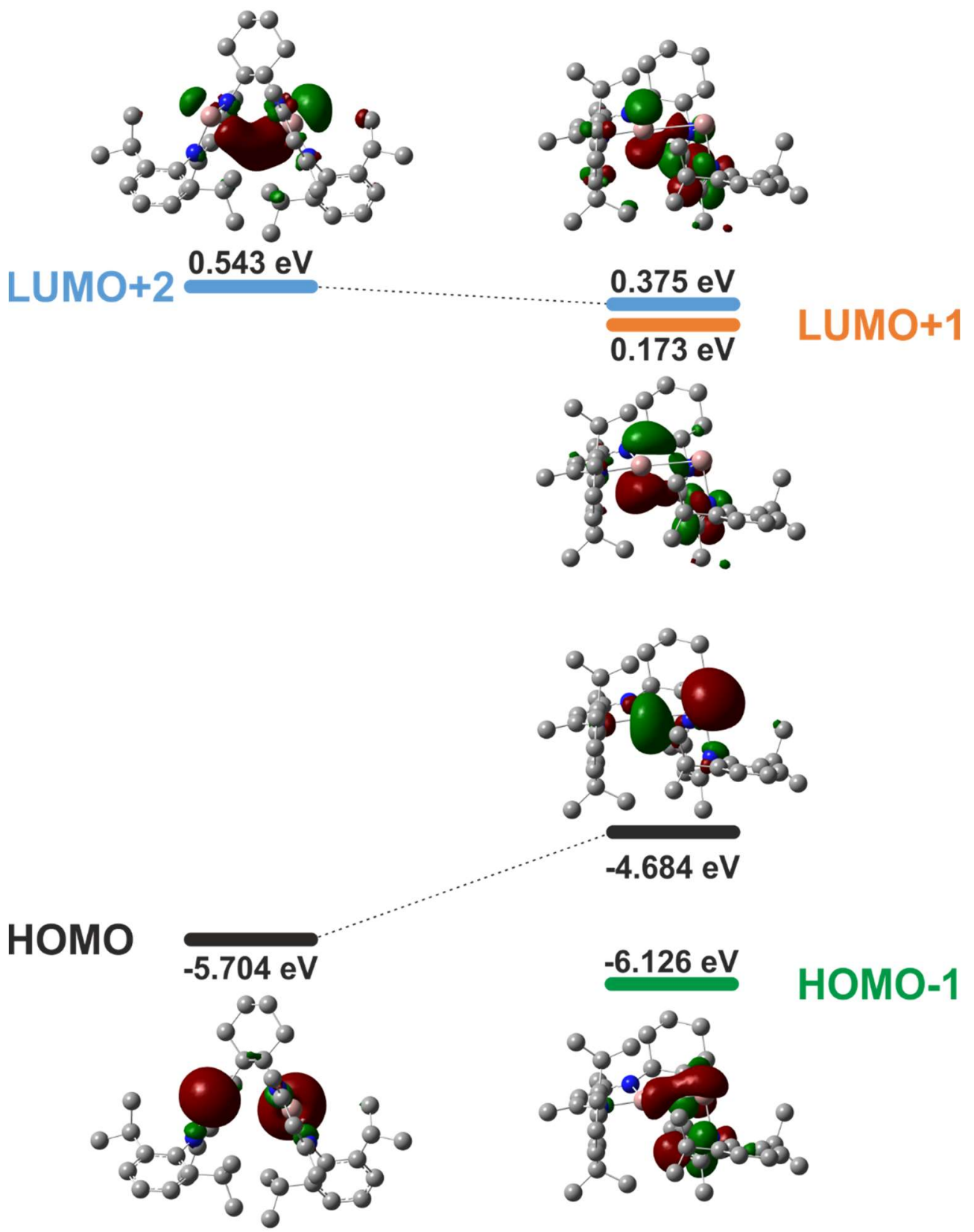

Figure S4. Frontier orbitals of 2 (left) and 2' (right) calculated at the M06-2X/6-31G(d) level of theory. 
Alternative reaction mechanism: Starting from 2, C-F bond activation by forming the $\mathrm{Ga}(\mathrm{I}) / \mathrm{Ga}(\mathrm{III})$ complex 6 can take place in a concerted manner at only one gallylene center via transition structure TS4, similar to previous reports about C-F bond activation by a mononuclear aluminium(I) $\beta$ diketiminate, S22 Figure S5. As 6 possesses an intact gallylene center, activation of a second fluoroarene molecule could occur in a similar fashion via Int4 and TS5 affording the dinuclear gallium(III)complex $\mathbf{7}$. In line with the experimental findings, the formation of $\mathbf{6}$ and $\mathbf{7}$ is impeded by substantial barriers $>147$ $\mathrm{kJ} \mathrm{mol}^{-1}$ ) in spite of being exergonic. As the formation of 6 is inhibited by the high energy demands of TS4, its involvement in the formation of 4 is unlikely. Nevertheless, we attempted to locate a pathway associated with the intramolecular attack of the gallium(III) center by the gallylene but could not identify any reasonable transition structure.

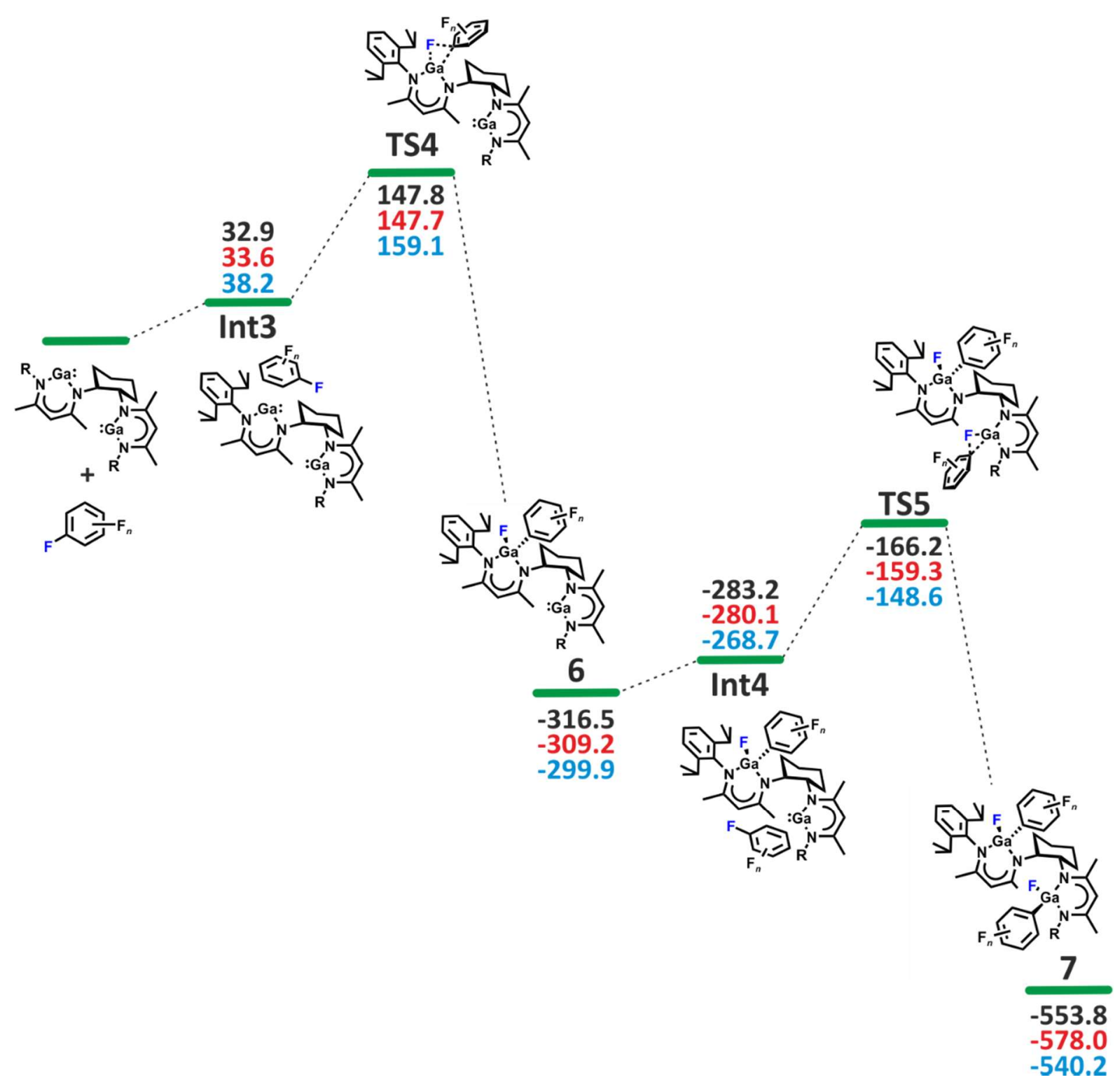

Figure S5. Schematic potential-energy surface with Gibbs free energies (calculated at the DLPNO$\operatorname{CCSD}(\mathrm{T}) / \mathrm{cc}-\mathrm{pVTZ} / / \mathrm{M} 06-2 \mathrm{X} / 6-31 \mathrm{G}$ (d) level of theory and given in $\mathrm{kJ} \mathrm{mol}^{-1}$ ) for the reaction of 2 with $\mathrm{C}_{6} \mathrm{~F}_{6}$ (a, black), $\mathrm{C}_{6} \mathrm{HF}_{5}$ (b, red) and 1,2,3,4- $\mathrm{C}_{6} \mathrm{H}_{2} \mathrm{~F}_{2}$ (c, blue) involving only a single metal center; $\mathrm{R}=\mathrm{Dipp}=$ 2,6-diisopropylphenyl. 
Mechanism of the $\mathrm{C}-\mathrm{F}$ bond activation brought about by 3 : The observed intermediates and transition structures, Figure S6, are reminiscent of those reported before for the aluminium(I) $\beta$ diketiminate A $(\mathrm{M}=\mathrm{Al}) .{ }^{\mathrm{S} 22}$ Similar to Int1", Int3, and Int4, noncovalent $\mathrm{C}-\mathrm{H} \cdots \mathrm{F}-\mathrm{C}$ contacts are also characteristic for Int5, while the subsequent three-membered transition structure TS6 resembles geometrical features of TS4 and TS5. Hence, $\mathrm{Ga}-\mathrm{C}$ and $\mathrm{Ga}-\mathrm{F}$ bond making and $\mathrm{C}-\mathrm{F}$ bond breaking take place in a single step. In line with the experimental findings, the energy of the respective transition structure increases in the order TS6a $<$ TS6b $<$ TS6c , which explains why the activation of 1,2,3,4tetrafluorobenzene (c) does not take place under the applied conditions.

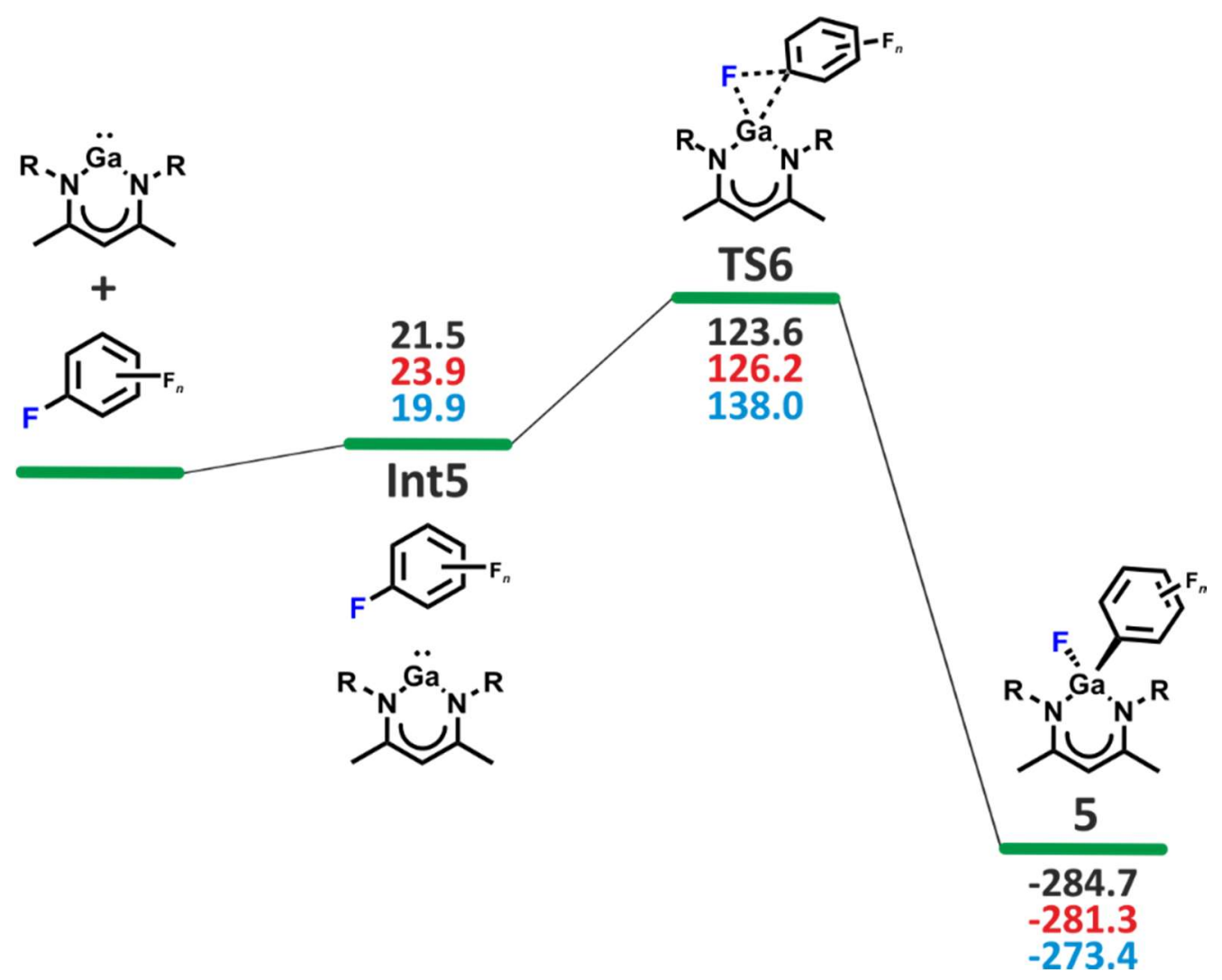

Figure S6. Schematic potential-energy with Gibbs free energies (calculated at the DLPNO-CCSD(T)/cc$\mathrm{pVTZ//M06-2X/6-31G(d)} \mathrm{level} \mathrm{of} \mathrm{theory} \mathrm{and} \mathrm{given} \mathrm{in} \mathrm{kJ} \mathrm{mol}^{-1}$ ) for the reaction of $\mathbf{3}$ with (a, black), $\mathrm{C}_{6} \mathrm{HF}_{5}$ (b, red) and 1,2,3,4- $\mathrm{C}_{6} \mathrm{H}_{2} \mathrm{~F}_{2}$ (c, blue); $\mathrm{R}=$ Dipp = 2,6-diisopropylphenyl. 


\section{NMR and IR spectra}

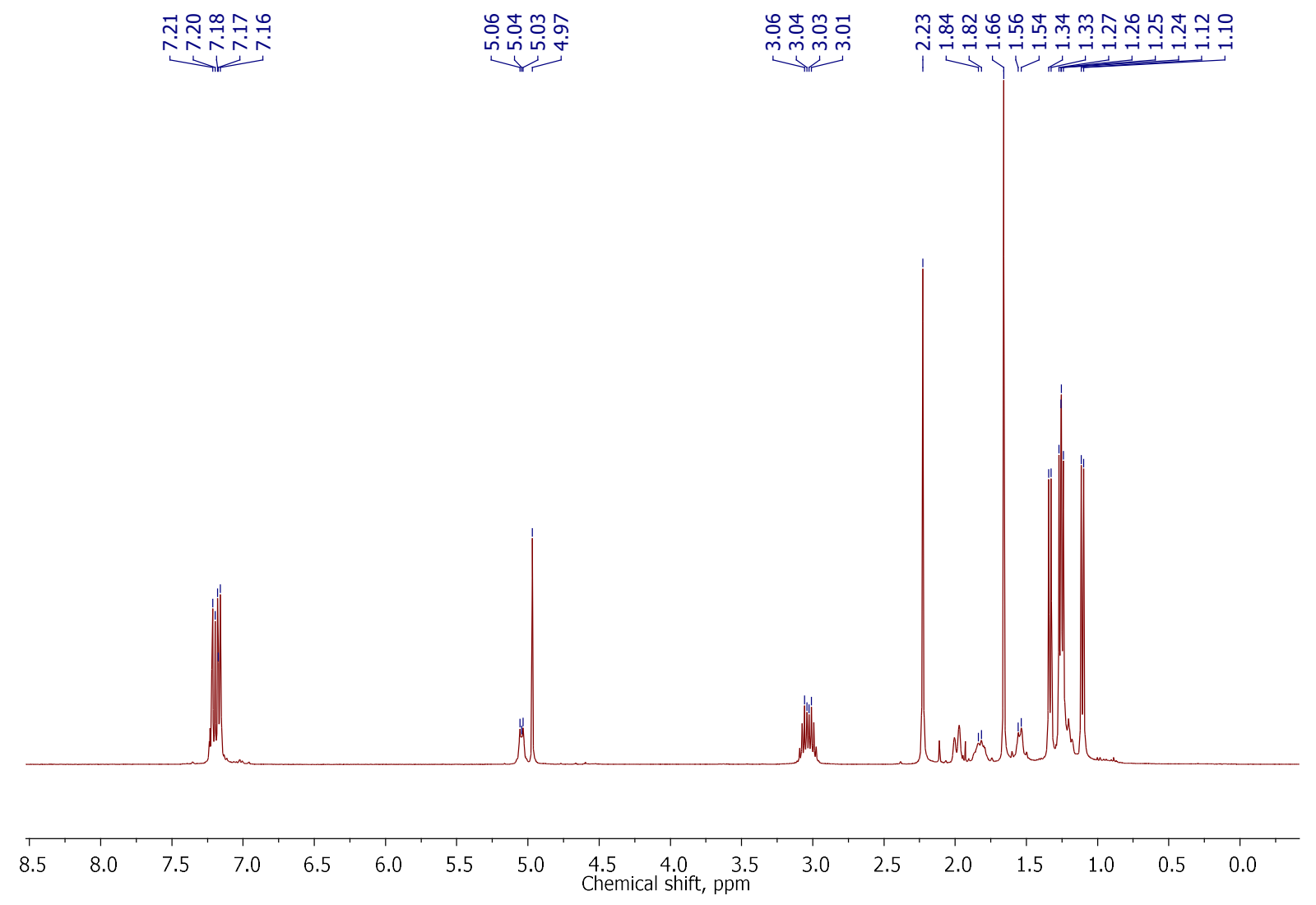

Figure S7. ${ }^{1} \mathrm{H}$ NMR spectrum $(600 \mathrm{MHz})$ of 2 in $\mathrm{C}_{6} \mathrm{D}_{6}$ (residual solvent peak at $\left.7.16 \mathrm{ppm}\right)$.

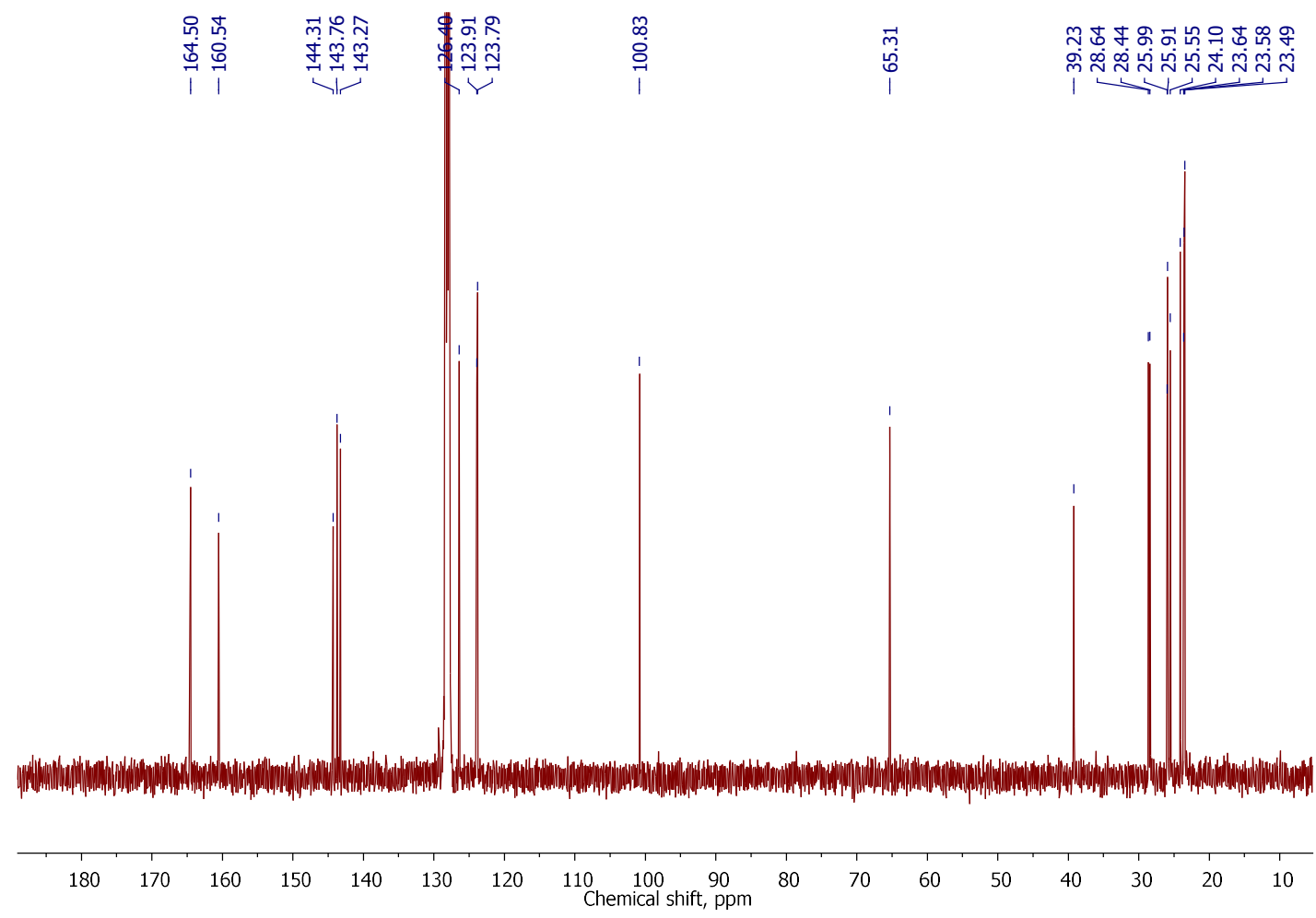

Figure S8. ${ }^{13} \mathrm{C}$ NMR spectrum (151 MHz) of 2 in $\mathrm{C}_{6} \mathrm{D}_{6}$ (solvent peak at $128.06 \mathrm{ppm}$ ). 

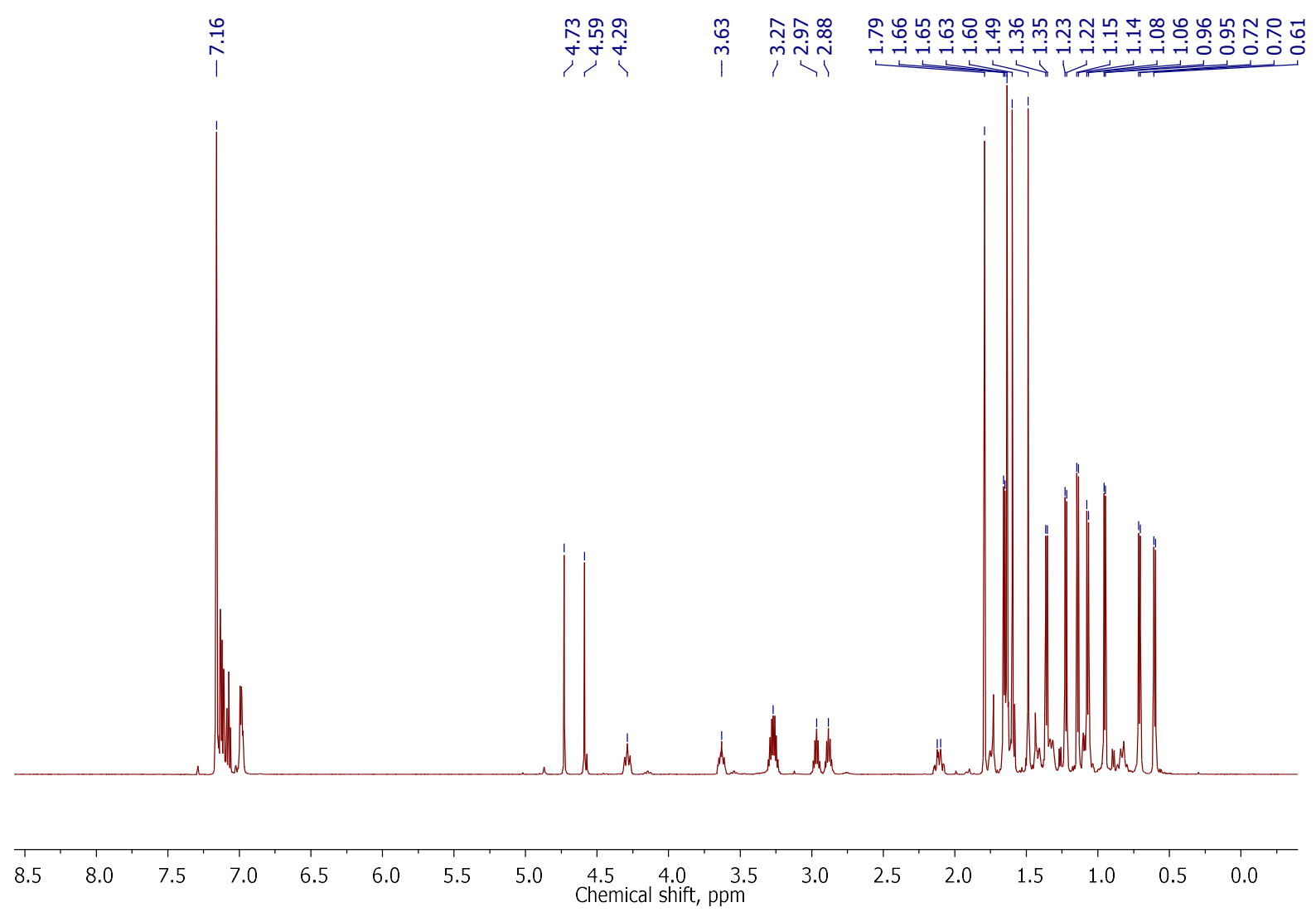

Figure S9. ${ }^{1} \mathrm{H}$ NMR spectrum $(600 \mathrm{MHz})$ of $4 \mathrm{a}$ in $\mathrm{C}_{6} \mathrm{D}_{6}$ (residual solvent peak at $7.16 \mathrm{ppm}$ ).

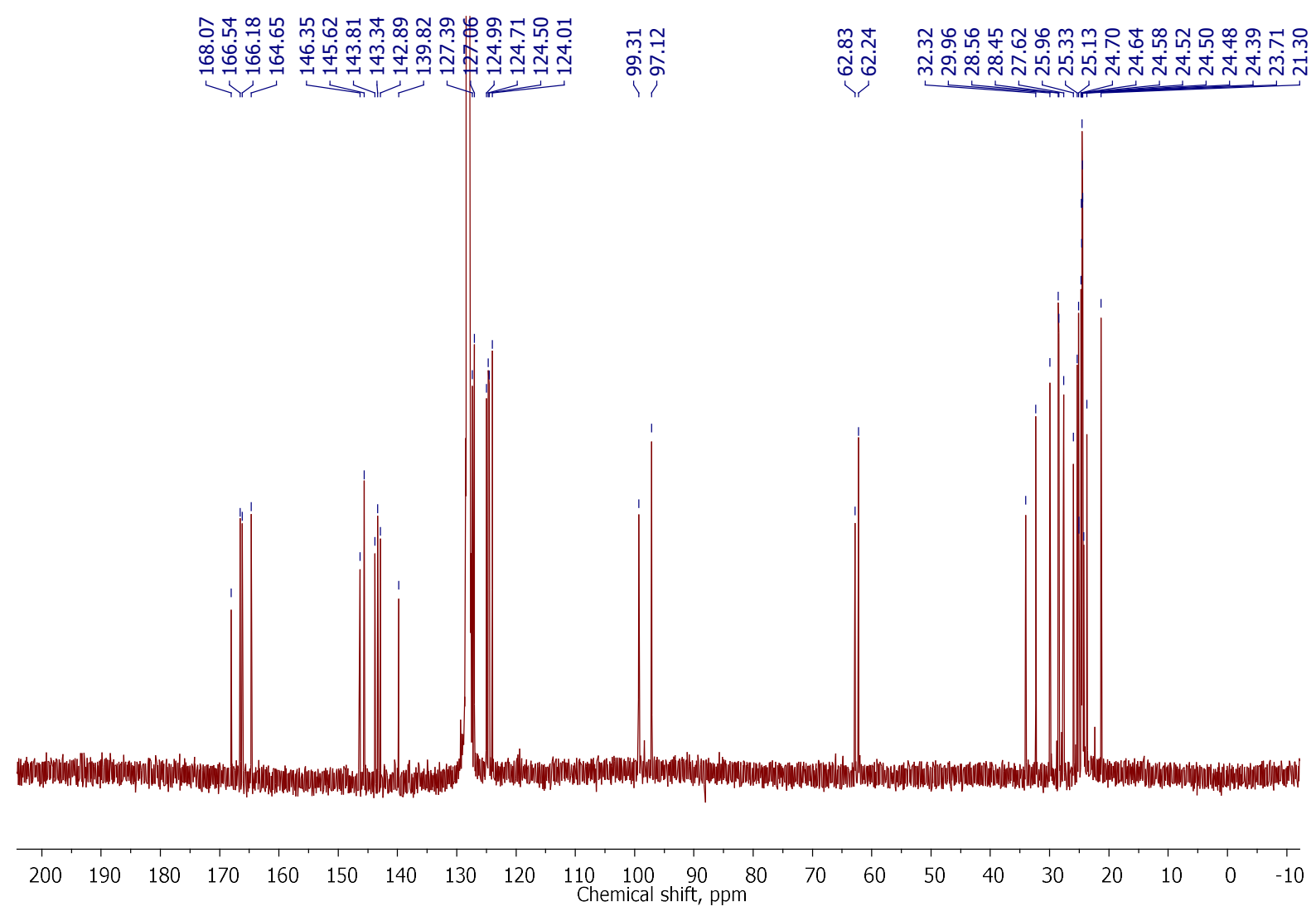

Figure S10. ${ }^{13} \mathrm{C}$ NMR spectrum (151 MHz) of $4 \mathrm{a}$ in $\mathrm{C}_{6} \mathrm{D}_{6}$ (solvent peak at $128.06 \mathrm{ppm}$ ). 


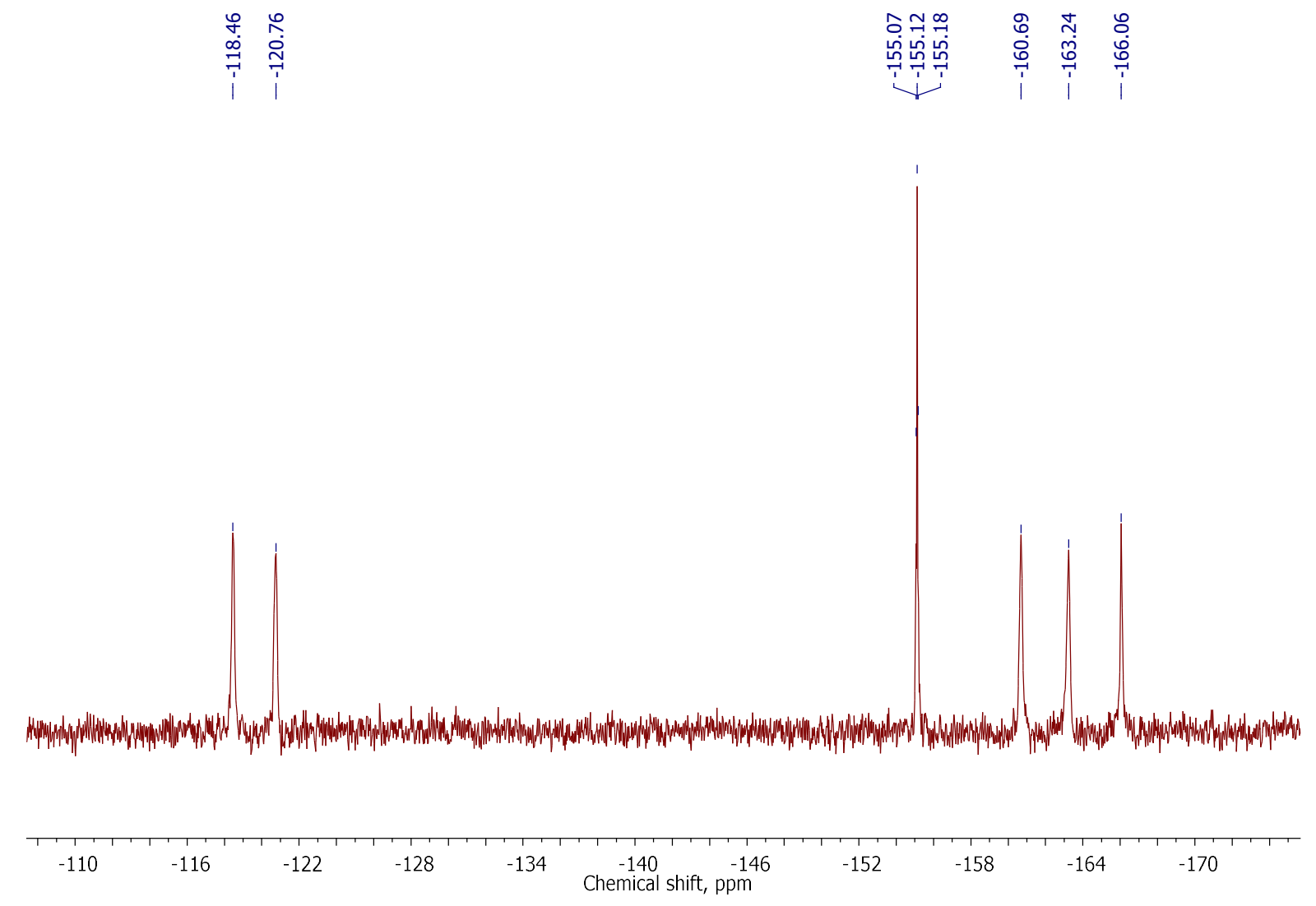

Figure S11. ${ }^{19} \mathrm{~F}$ NMR spectrum $(377 \mathrm{MHz})$ of $4 \mathrm{a}$ in $\mathrm{C}_{6} \mathrm{D}_{6}$.
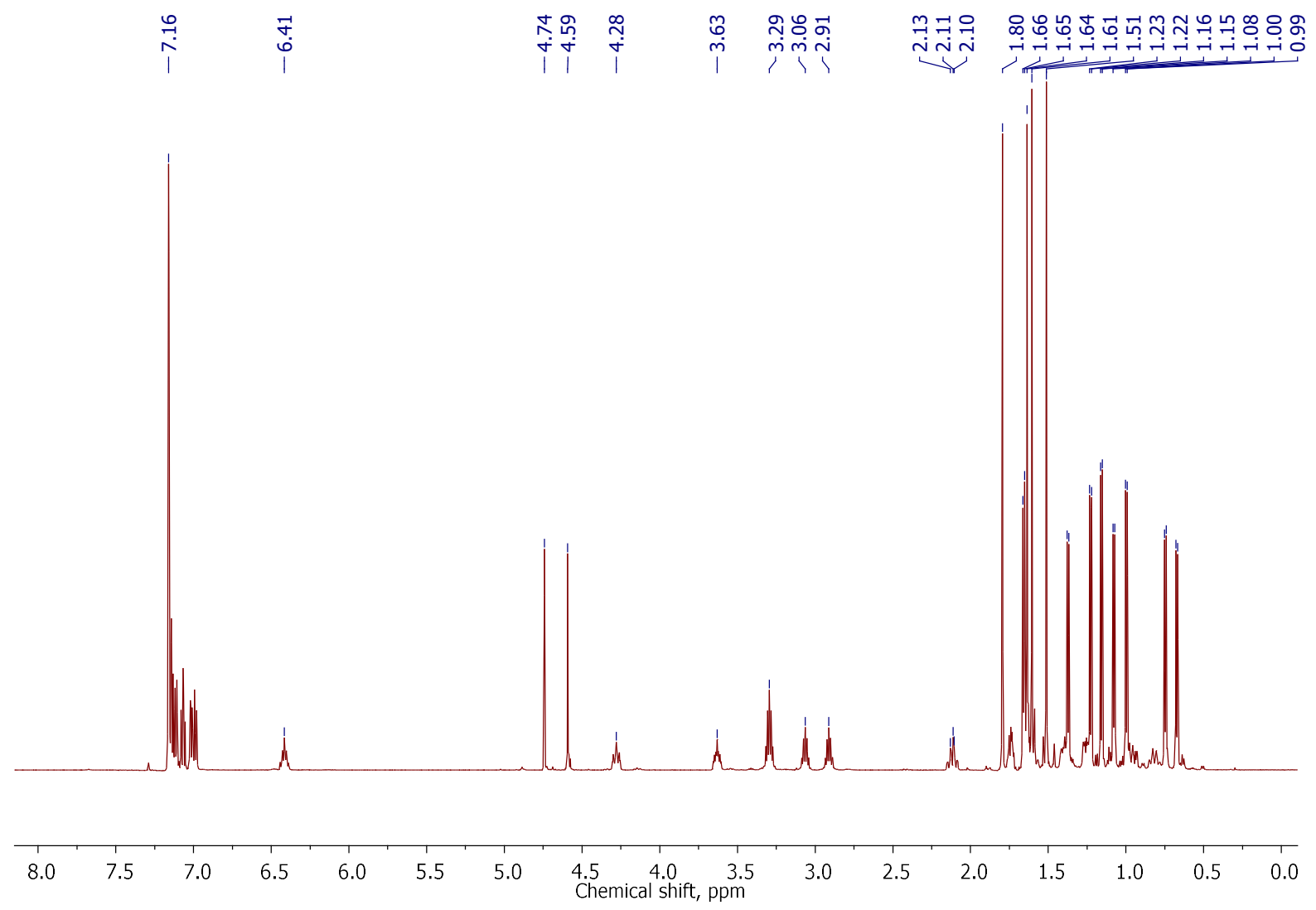

Figure S12. ${ }^{1} \mathrm{H}$ NMR spectrum $(600 \mathrm{MHz})$ of $\mathbf{4 b}$ in $\mathrm{C}_{6} \mathrm{D}_{6}$ (residual solvent peak at $7.16 \mathrm{ppm}$ ). 


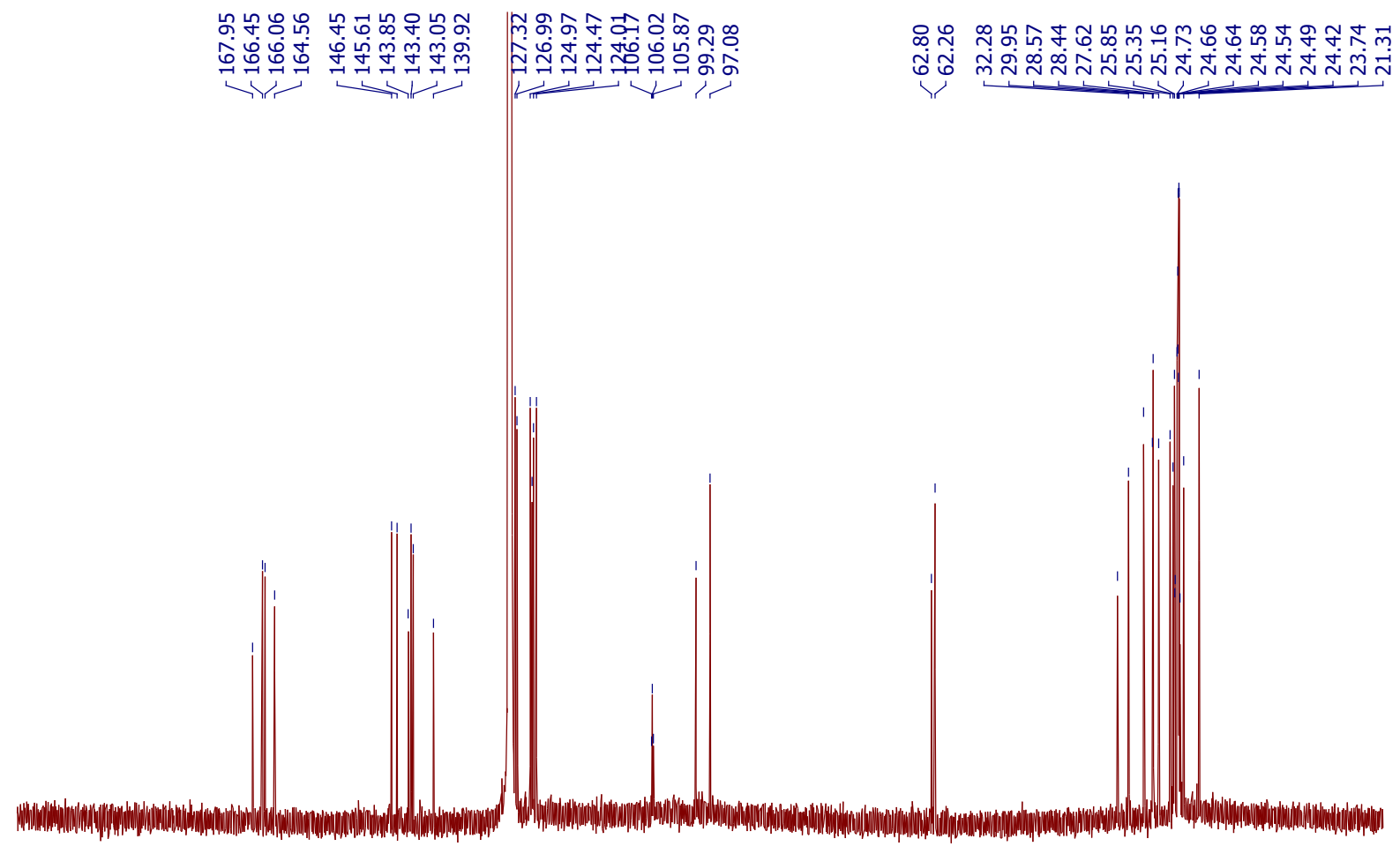

$\begin{array}{llllllllllllllllllll}200 & 190 & 180 & 170 & 160 & 150 & 140 & 130 & 120 & \begin{array}{c}110 \\ \text { Chemical shift, }\end{array} \text { ppm }\end{array}$

Figure $\mathbf{S} 13 .{ }^{13} \mathrm{C}$ NMR spectrum $(151 \mathrm{MHz})$ of $\mathbf{4 b}$ in $\mathrm{C}_{6} \mathrm{D}_{6}$ (solvent peak at $128.06 \mathrm{ppm}$ ).

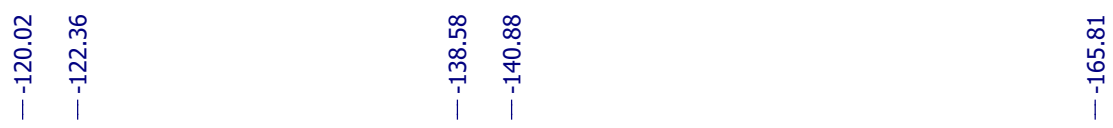

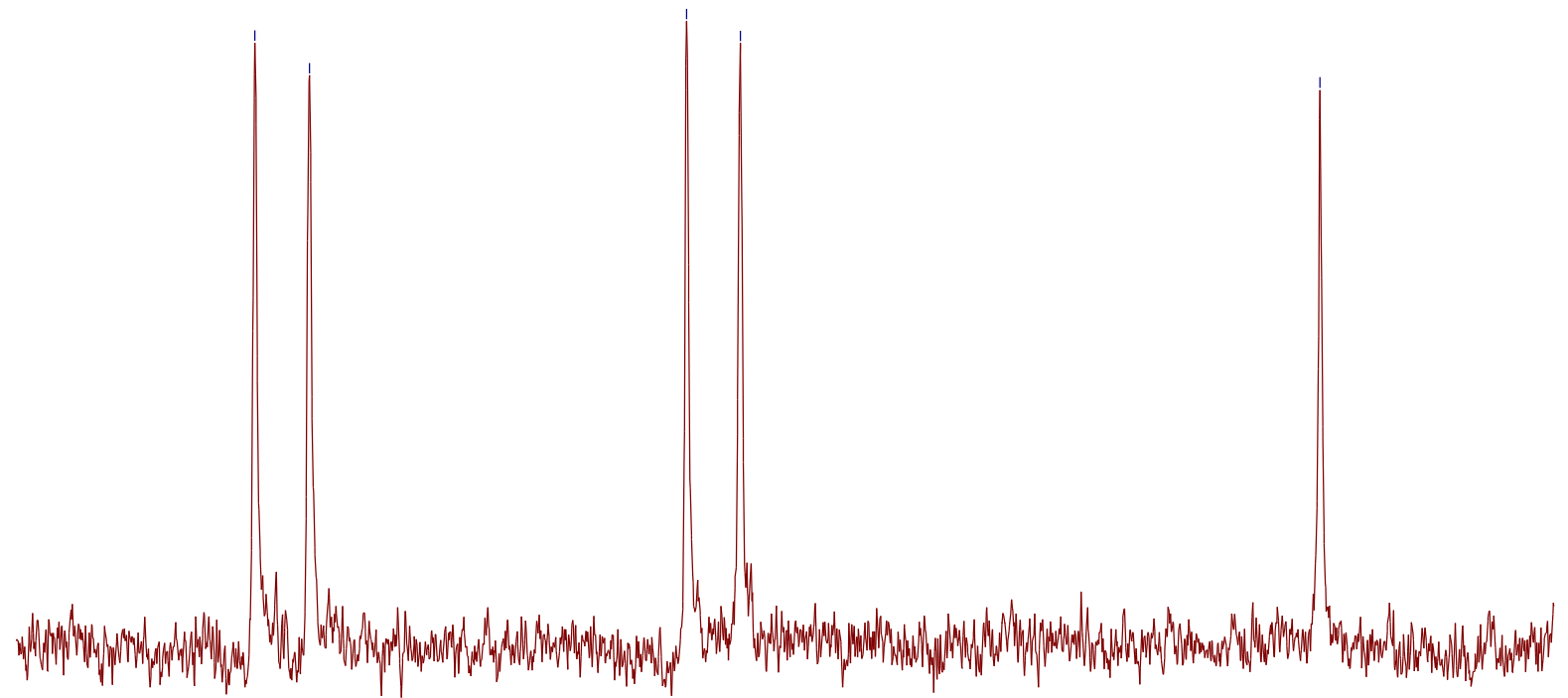

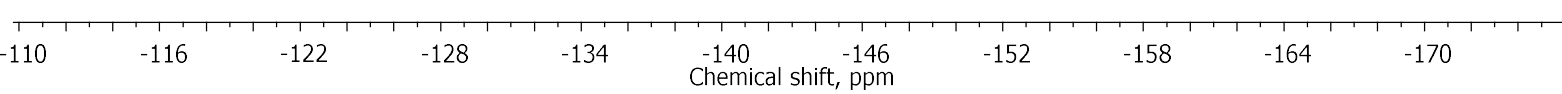

Figure S14. ${ }^{19} \mathrm{~F}$ NMR spectrum $(377 \mathrm{MHz})$ of $4 \mathbf{b}$ in $\mathrm{C}_{6} \mathrm{D}_{6}$. 

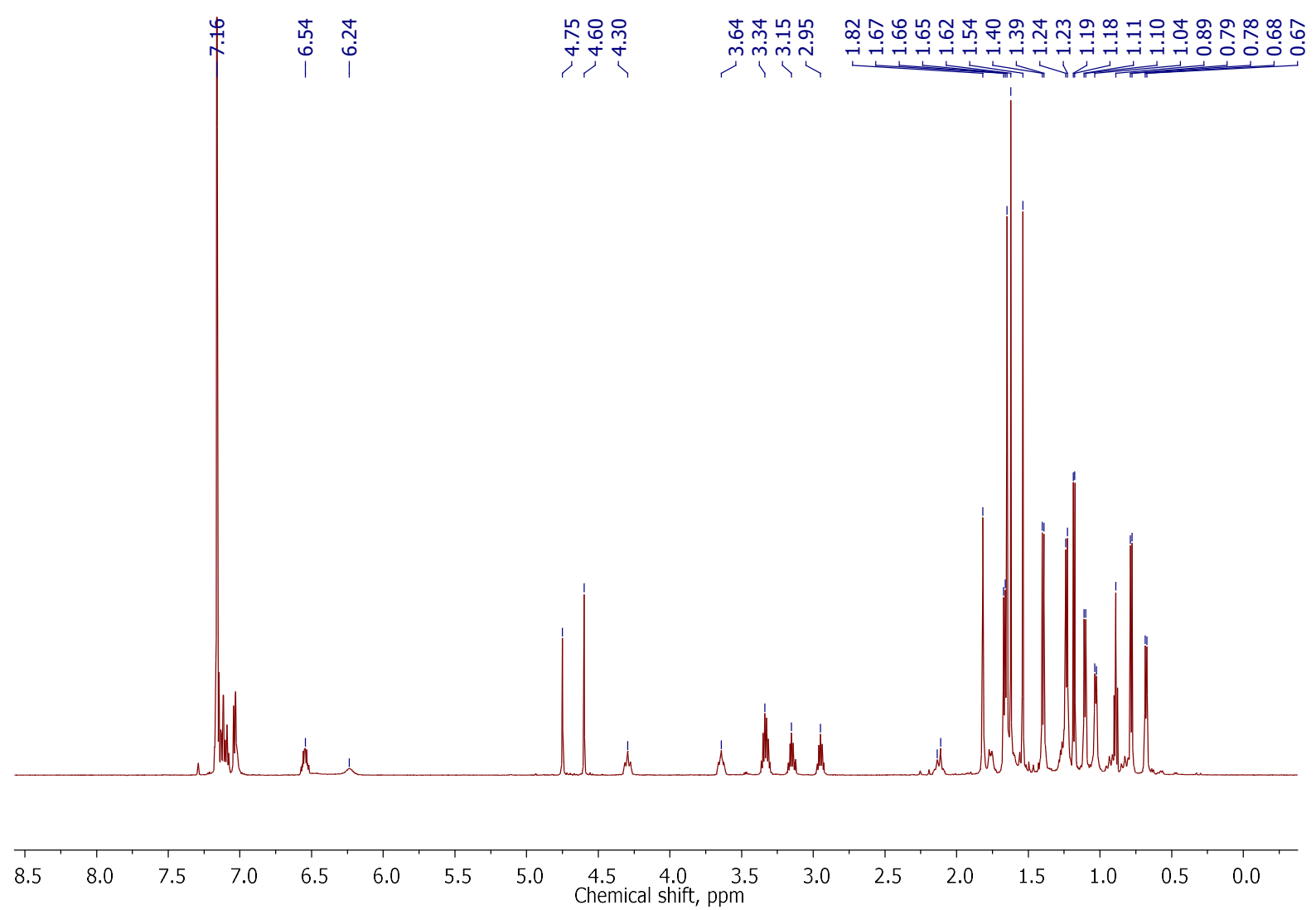

Figure S15. ${ }^{1} \mathrm{H}$ NMR spectrum $(600 \mathrm{MHz})$ of $4 \mathrm{c}$ in $\mathrm{C}_{6} \mathrm{D}_{6}$ (residual solvent peak at $7.16 \mathrm{ppm}$ ).

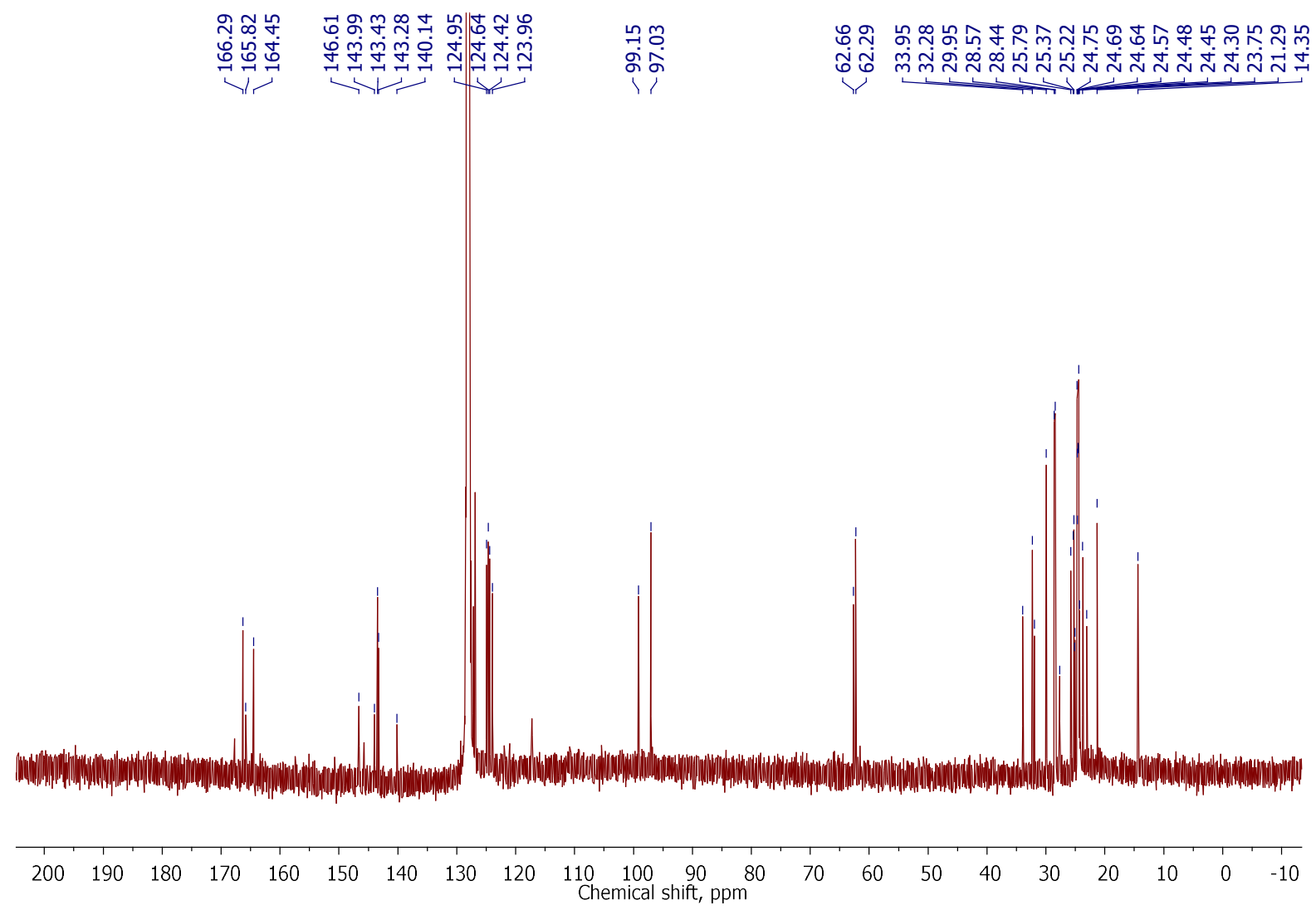

Figure S16. ${ }^{13} \mathrm{C}$ NMR spectrum (151 MHz) of $4 \mathrm{c}$ in $\mathrm{C}_{6} \mathrm{D}_{6}$ (solvent peak at $128.06 \mathrm{ppm}$ ). 


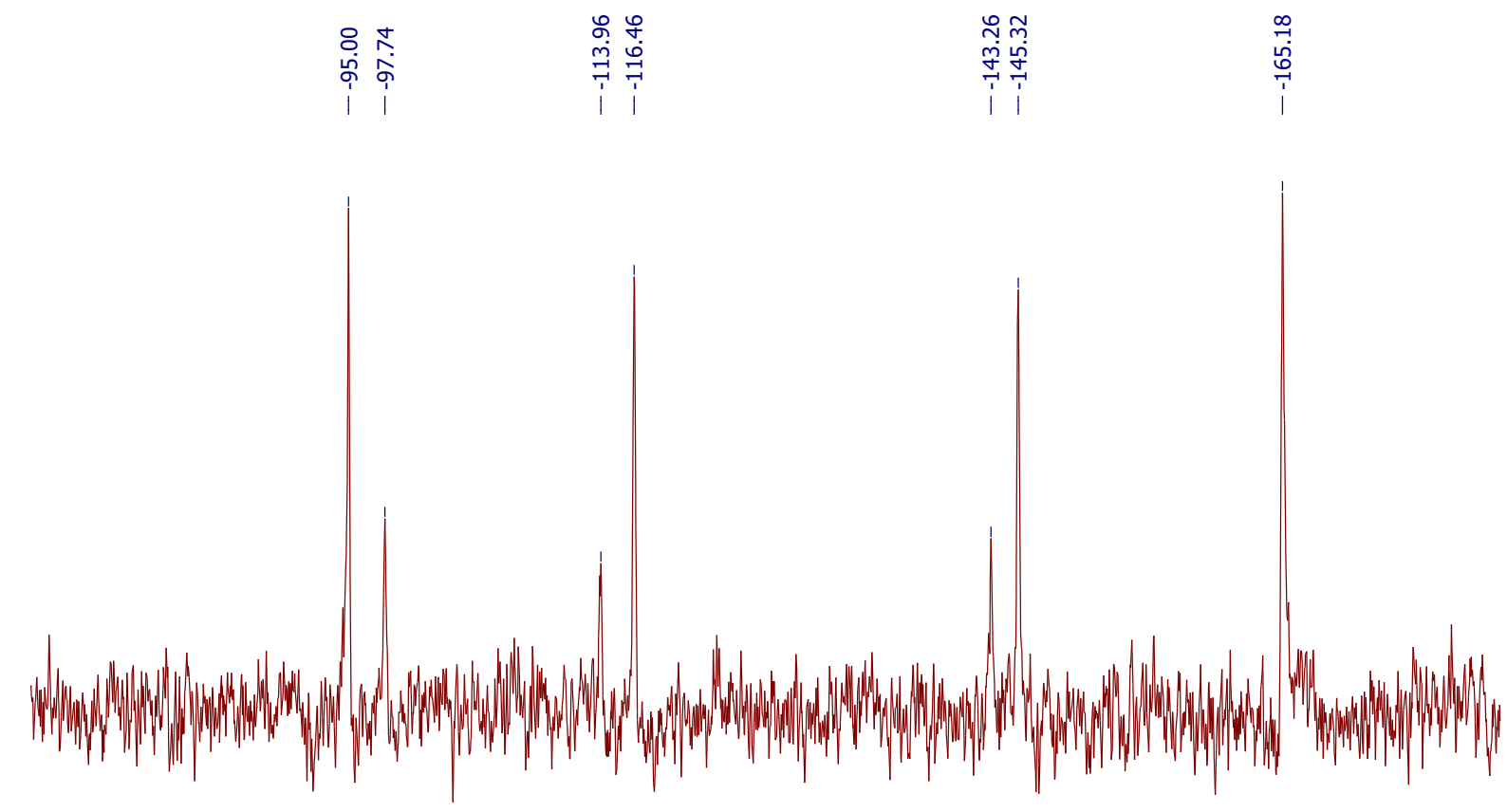

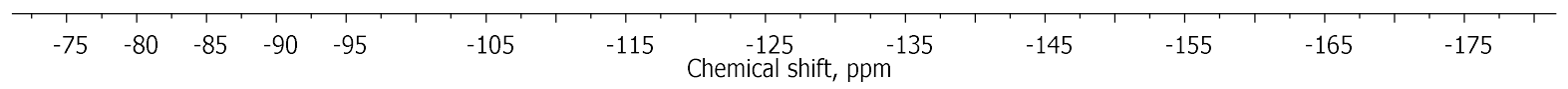

Figure S17. ${ }^{19} \mathrm{~F}$ NMR spectrum $(377 \mathrm{MHz})$ of $4 \mathrm{c}$ in $\mathrm{C}_{6} \mathrm{D}_{6}$.

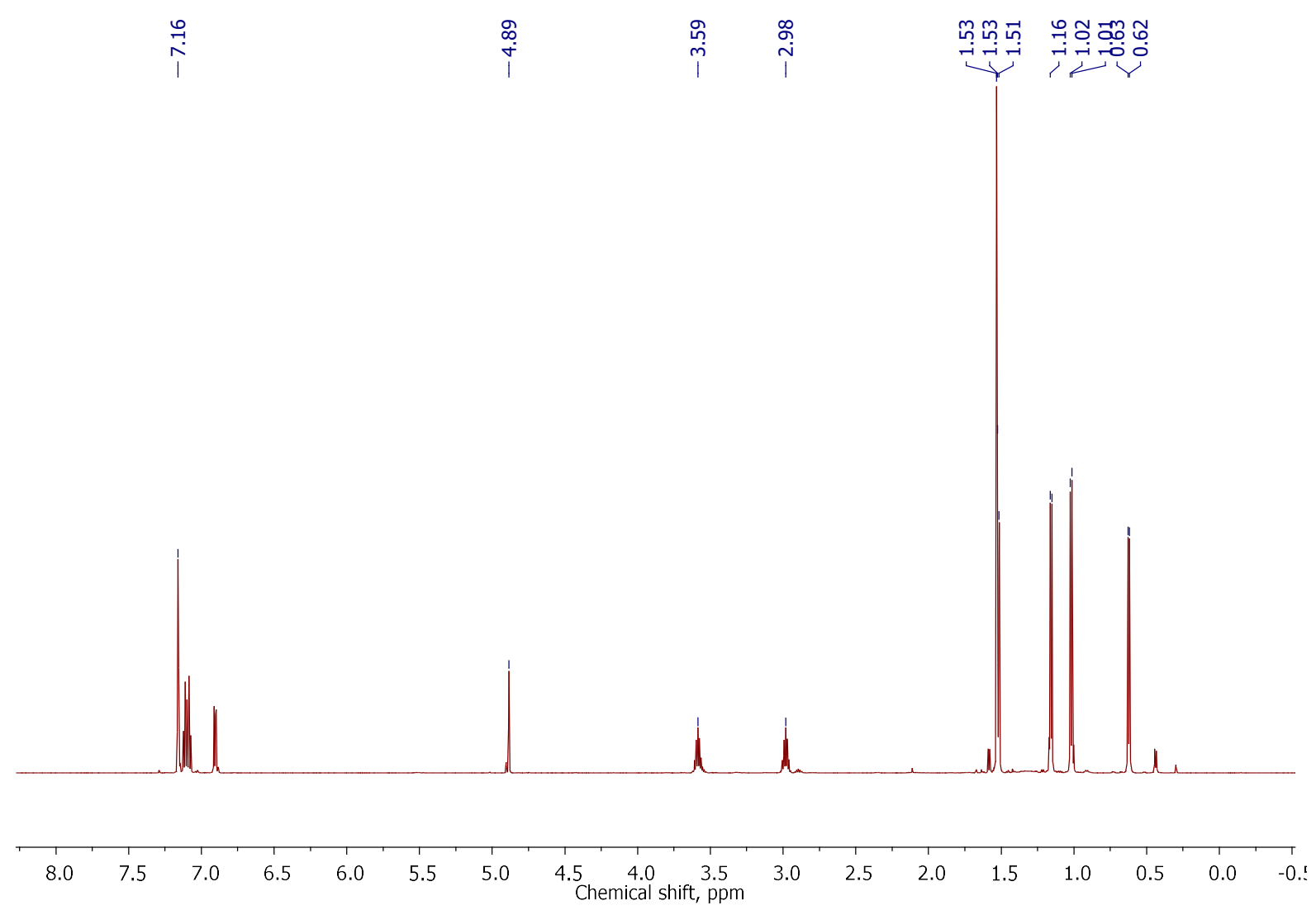

Figure S18. ${ }^{1} \mathrm{H}$ NMR spectrum $(600 \mathrm{MHz})$ of $\mathbf{5 a}$ in $\mathrm{C}_{6} \mathrm{D}_{6}$ (residual solvent peak at $7.16 \mathrm{ppm}$ ). 

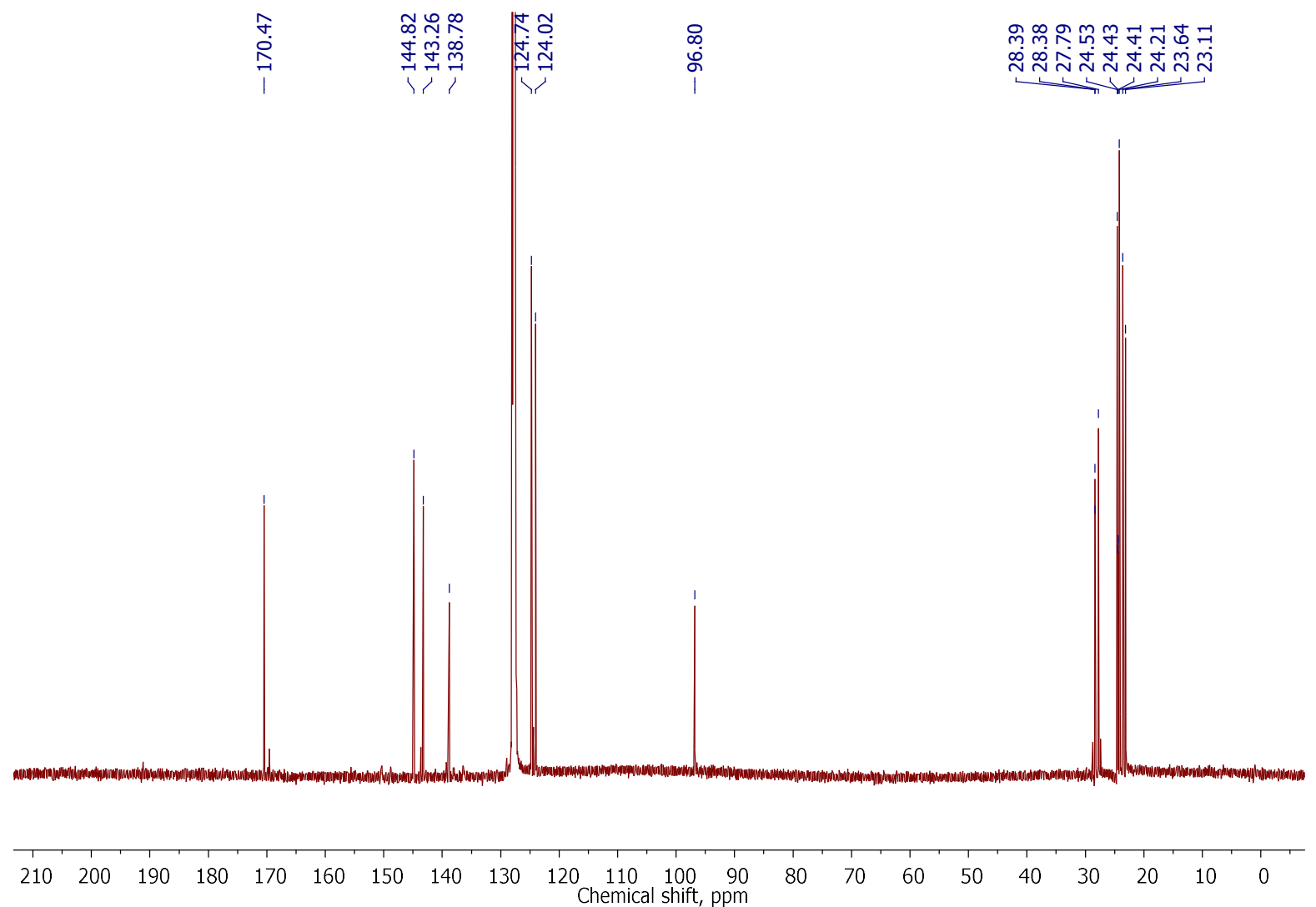

Figure S19. ${ }^{13} \mathrm{C}$ NMR spectrum (151 MHz) of $\mathbf{5 a}$ in $\mathrm{C}_{6} \mathrm{D}_{6}$ (solvent peak at $128.06 \mathrm{ppm}$ ).

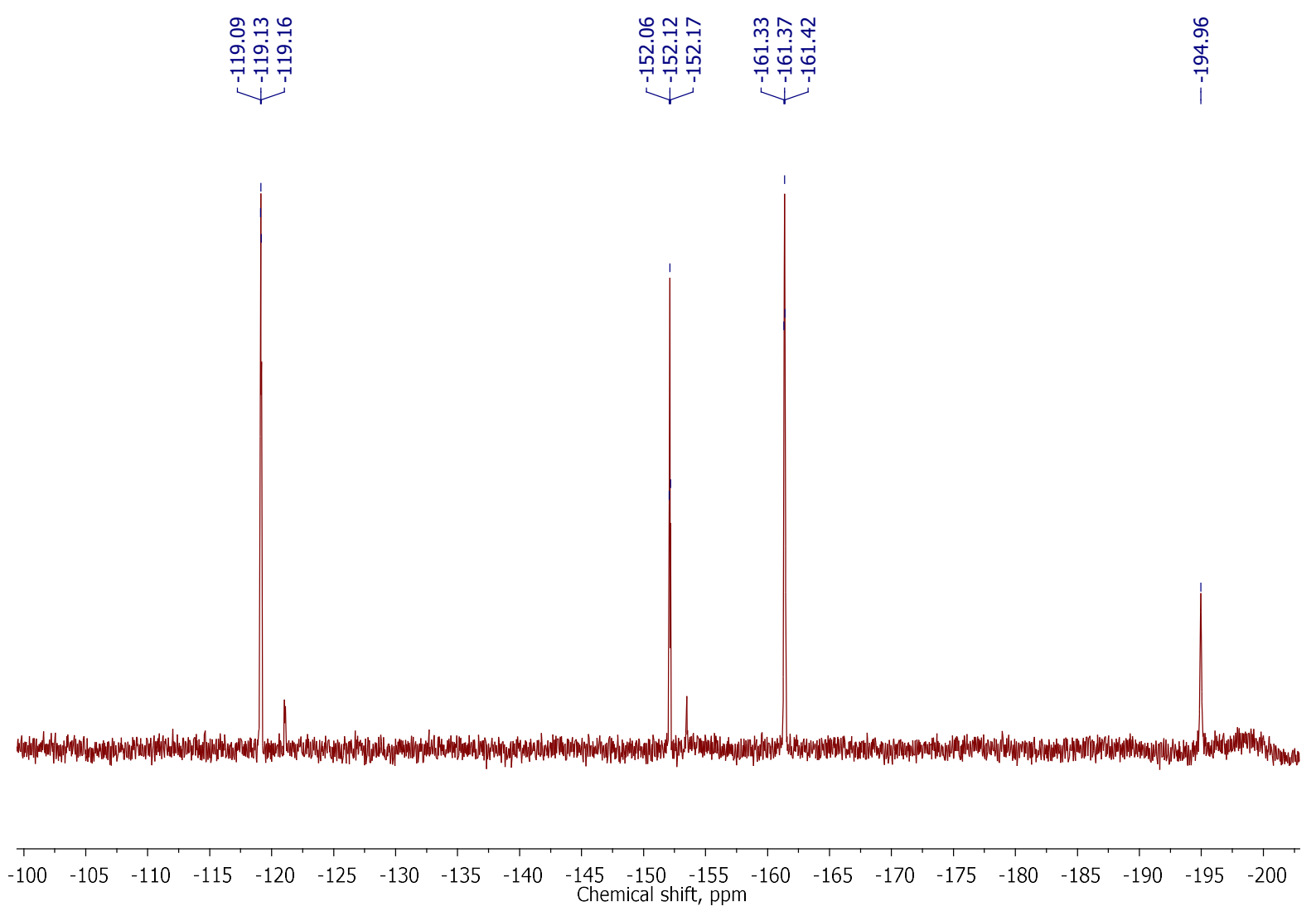

Figure S20. ${ }^{19} \mathrm{~F}$ NMR spectrum $(377 \mathrm{MHz})$ of $5 \mathrm{a}$ in $\mathrm{C}_{6} \mathrm{D}_{6}$. 


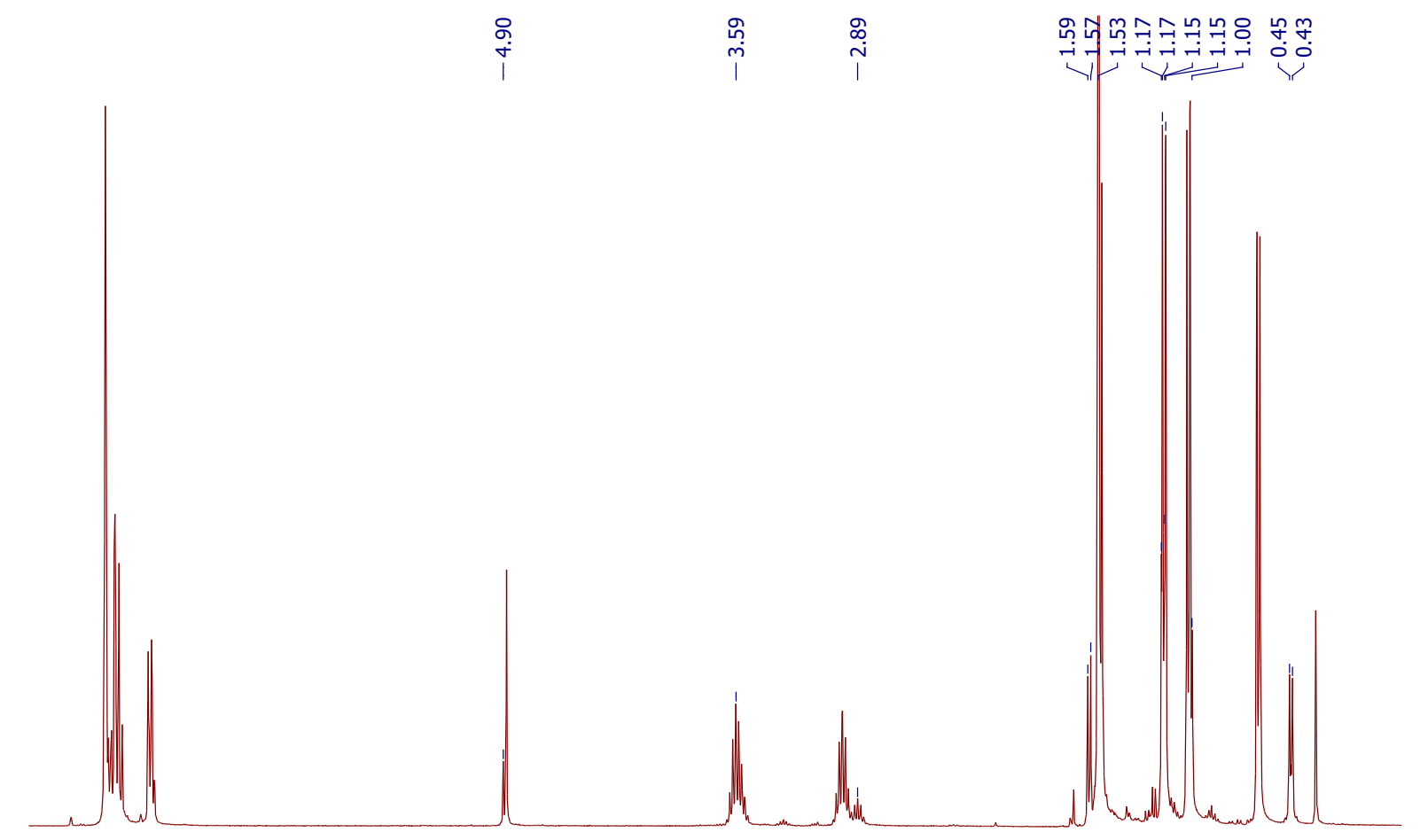

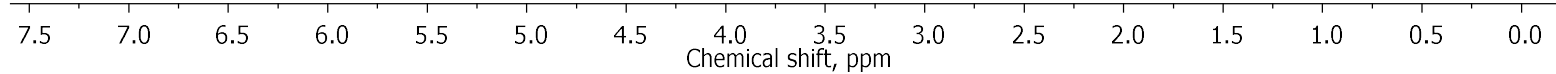

Figure S21. ${ }^{1} \mathrm{H}$ NMR spectrum $(400 \mathrm{MHz})$ of the product of full scale synthesis of $\mathbf{5 a}$ indicating presence of a side-product in $\mathrm{C}_{6} \mathrm{D}_{6}$ (residual solvent peak at $7.16 \mathrm{ppm}$ ). Marked are the peaks which belong to the side-product. 


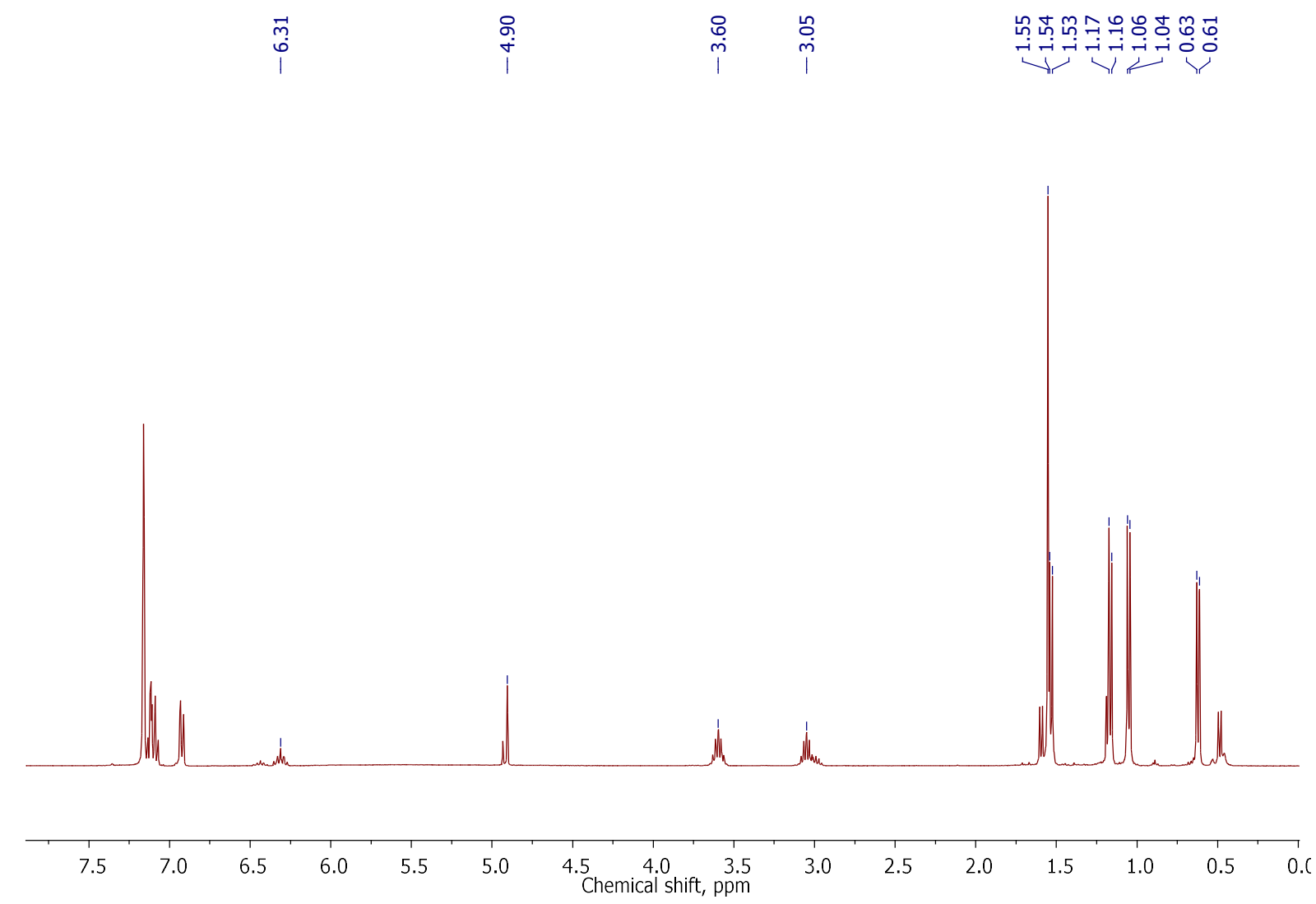

Figure S22. ${ }^{1} \mathrm{H}$ NMR spectrum $(400 \mathrm{MHz})$ of the product of full scale synthesis of $\mathbf{5 b}$ indicating presence of a side-product in $\mathrm{C}_{6} \mathrm{D}_{6}$ (residual solvent peak at $7.16 \mathrm{ppm}$ ). Marked are the peaks which belong to the main product $\mathbf{5 b}$. 


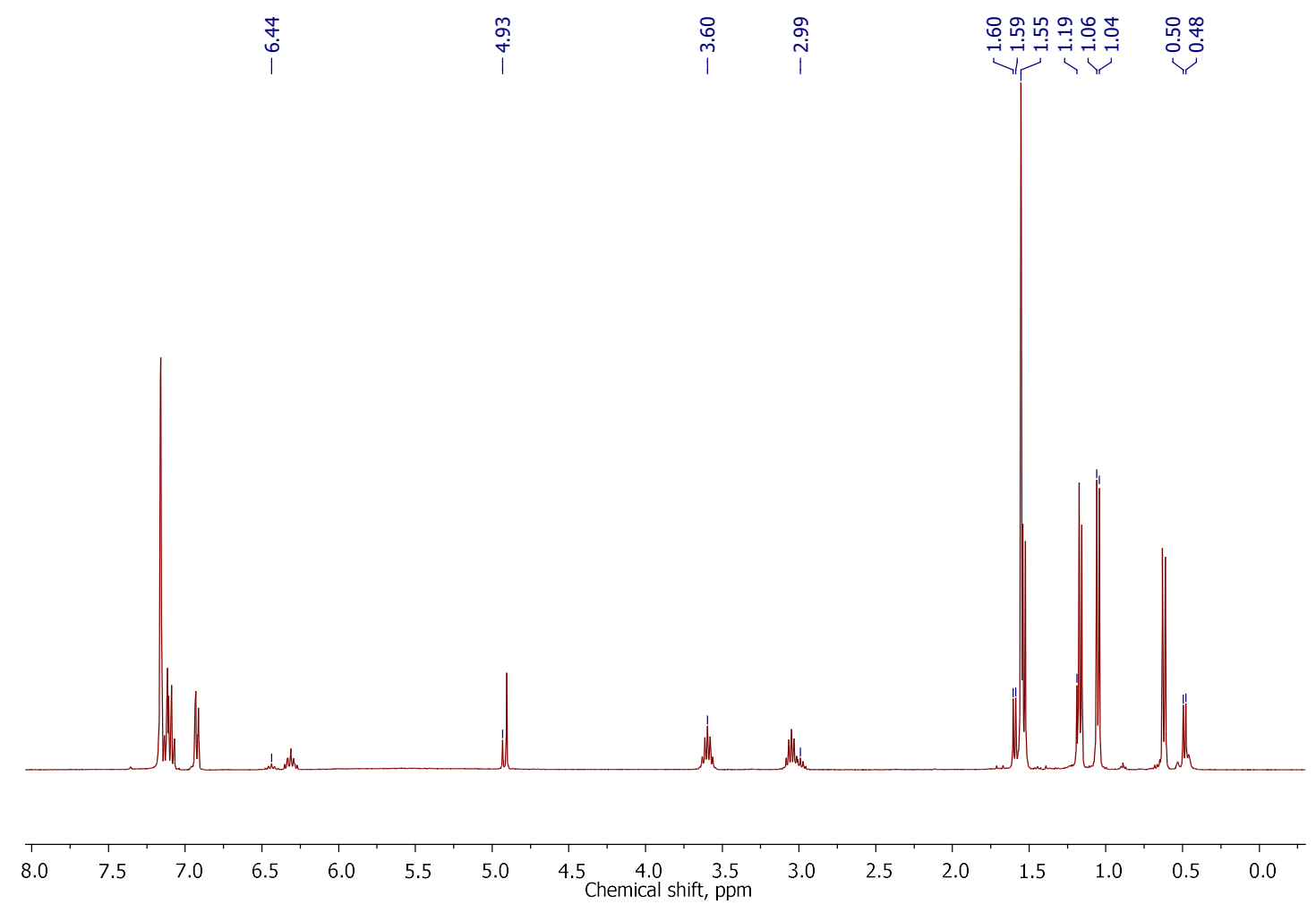

Figure S23. ${ }^{1} \mathrm{H}$ NMR spectrum $(400 \mathrm{MHz})$ of the product of full scale synthesis of $\mathbf{5} \mathbf{b}$ indicating presence of a side-product in $\mathrm{C}_{6} \mathrm{D}_{6}$ (residual solvent peak at $7.16 \mathrm{ppm}$ ). Marked are the peaks which belong to the side-product.

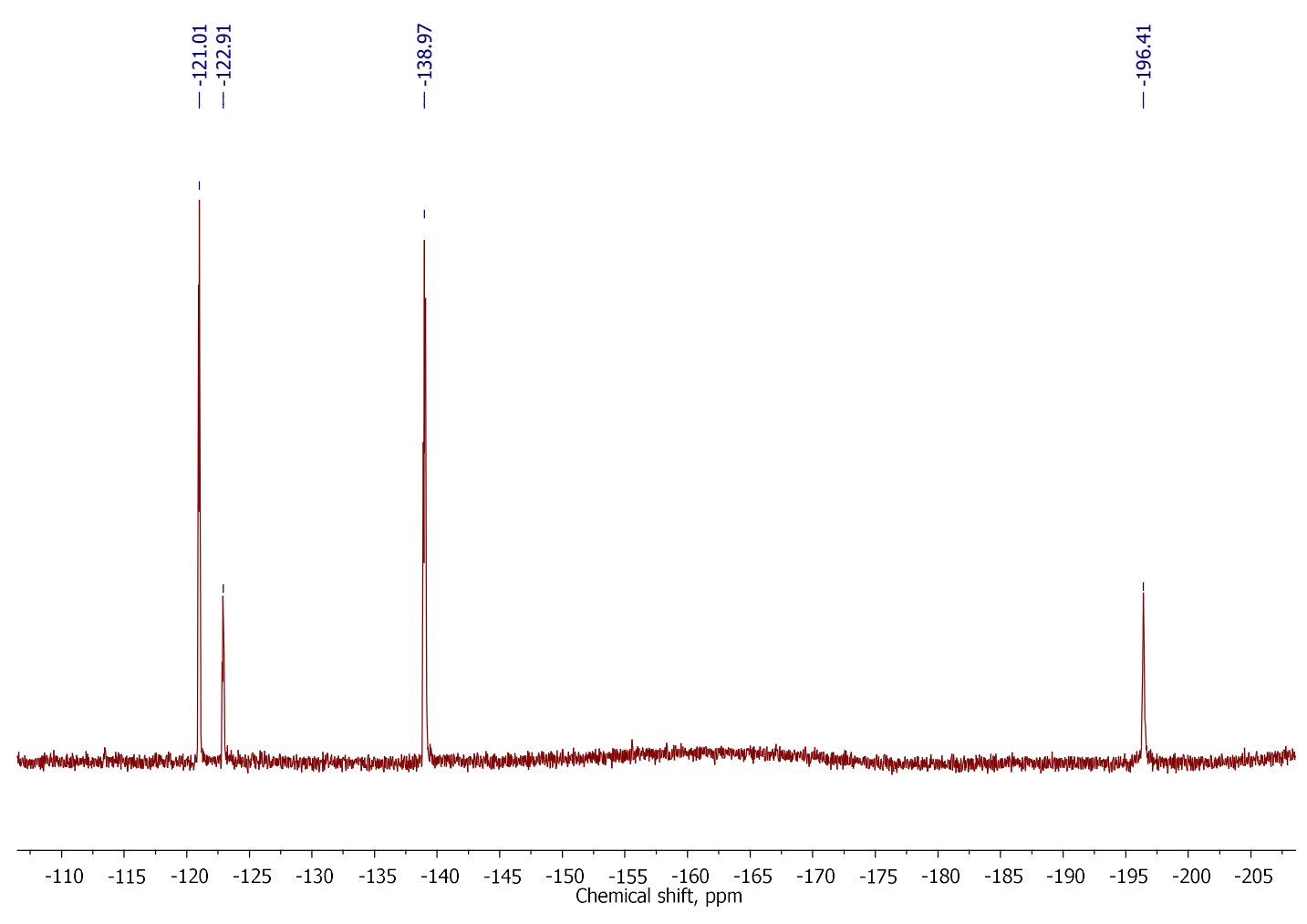

Figure S24. ${ }^{19} \mathrm{~F}$ NMR spectrum $(288 \mathrm{MHz})$ of the product of full scale synthesis of $\mathbf{5 b}$ indicating presence of a side-product (-122.9 ppm) in $\mathrm{C}_{6} \mathrm{D}_{6}$. 


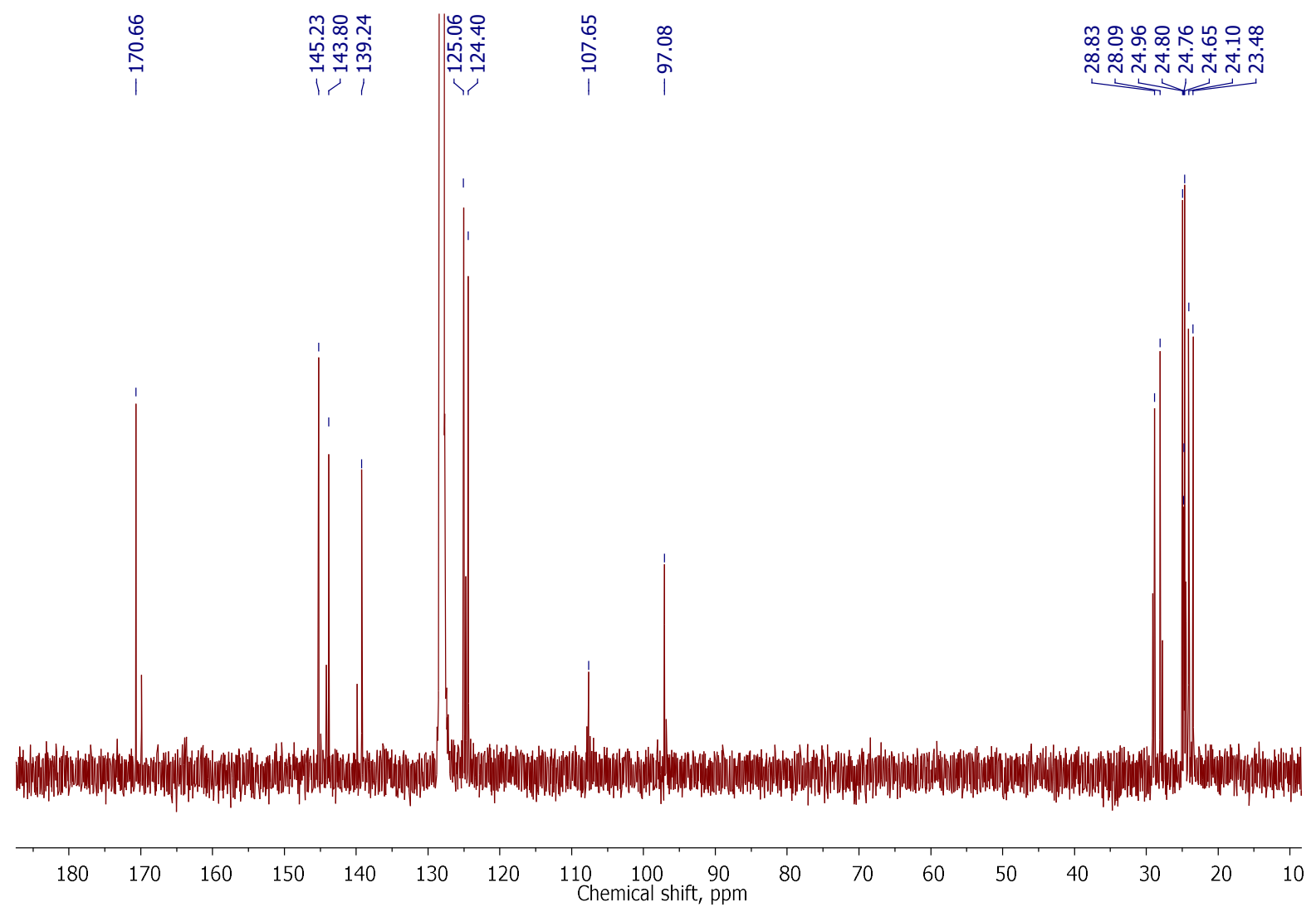

Figure S25. ${ }^{13} \mathrm{C}$ NMR spectrum (151 MHz) of the product of full scale synthesis of $\mathbf{5 b}$ in $\mathrm{C}_{6} \mathrm{D}_{6}$ (solvent peak at $128.06 \mathrm{ppm})$.

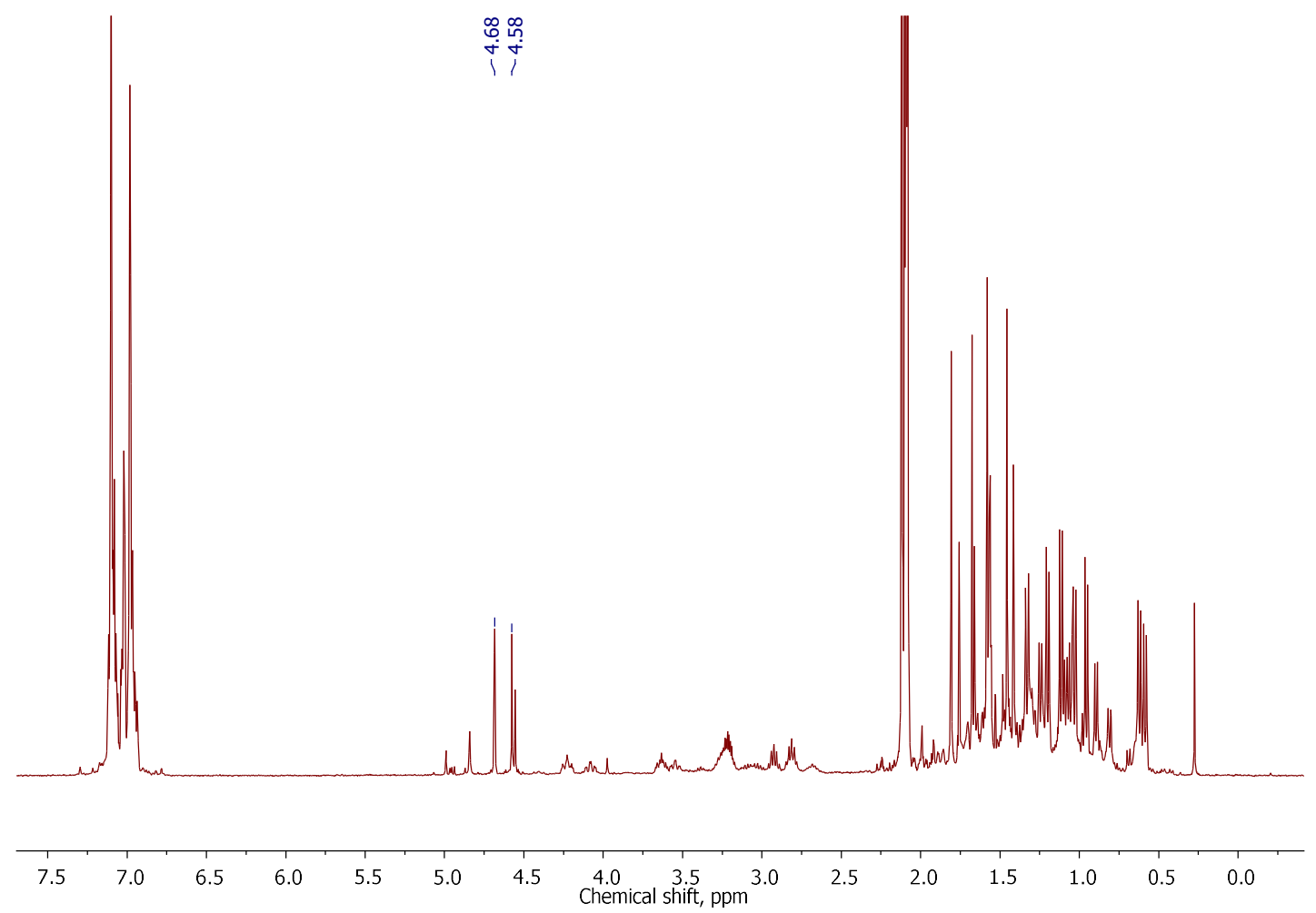

Figure S26. ${ }^{1} \mathrm{H}$ NMR spectrum $(400 \mathrm{MHz})$ of the final reaction mixture from the synthesis of $4 \mathrm{a}$ in toluene- $\mathrm{d}_{8}$. Marked are the characteristic peaks of $\mathbf{4 a}(\mathrm{CH}$ methine). 2 is fully consumed. 


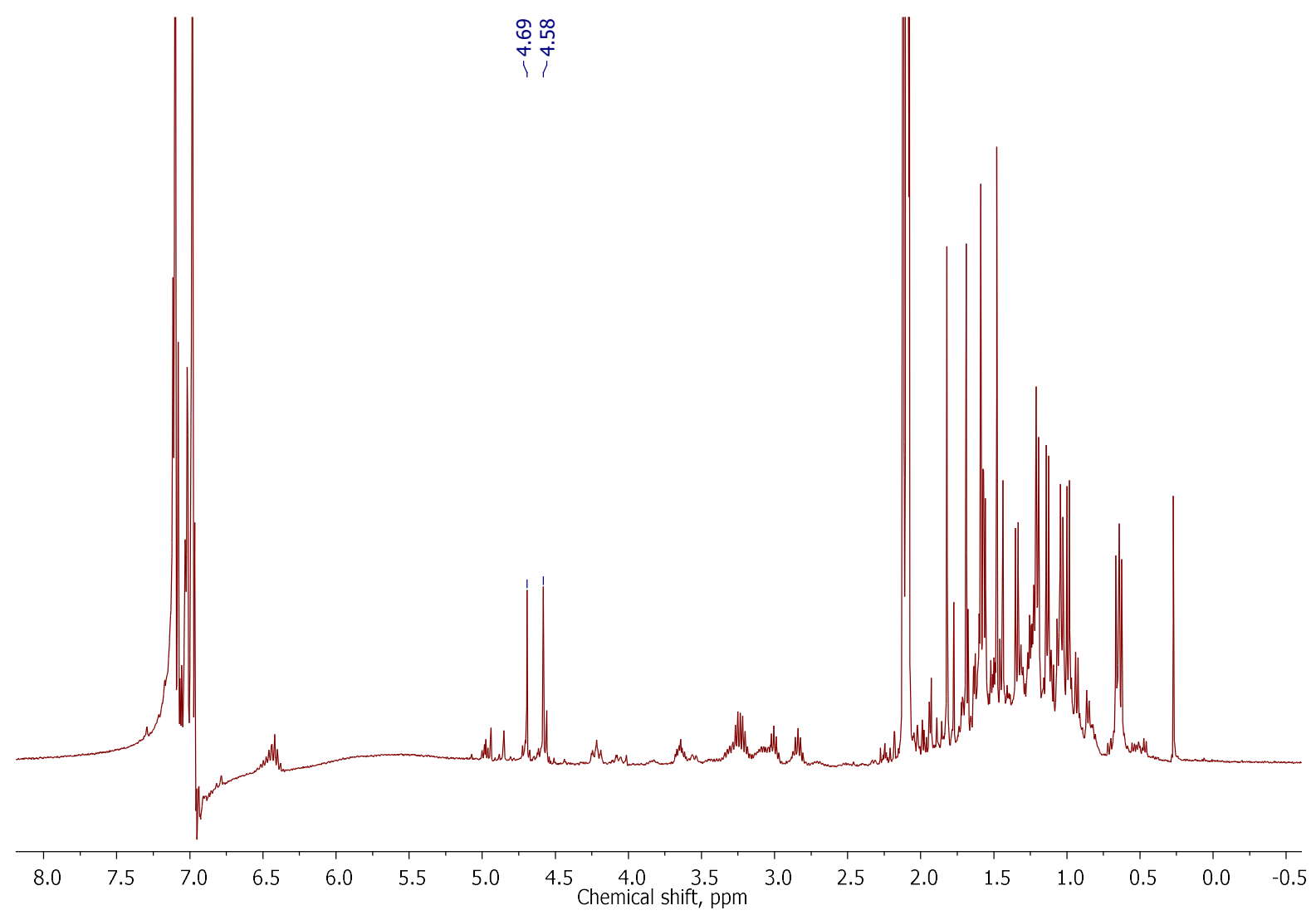

Figure S27. ${ }^{1} \mathrm{H}$ NMR spectrum $(400 \mathrm{MHz})$ of the final reaction mixture from the synthesis of $4 \mathbf{b}$ in toluene- $d_{8}$. Marked are the characteristic peaks of $4 b$ ( $\mathrm{CH}$ methine). 2 is fully consumed.

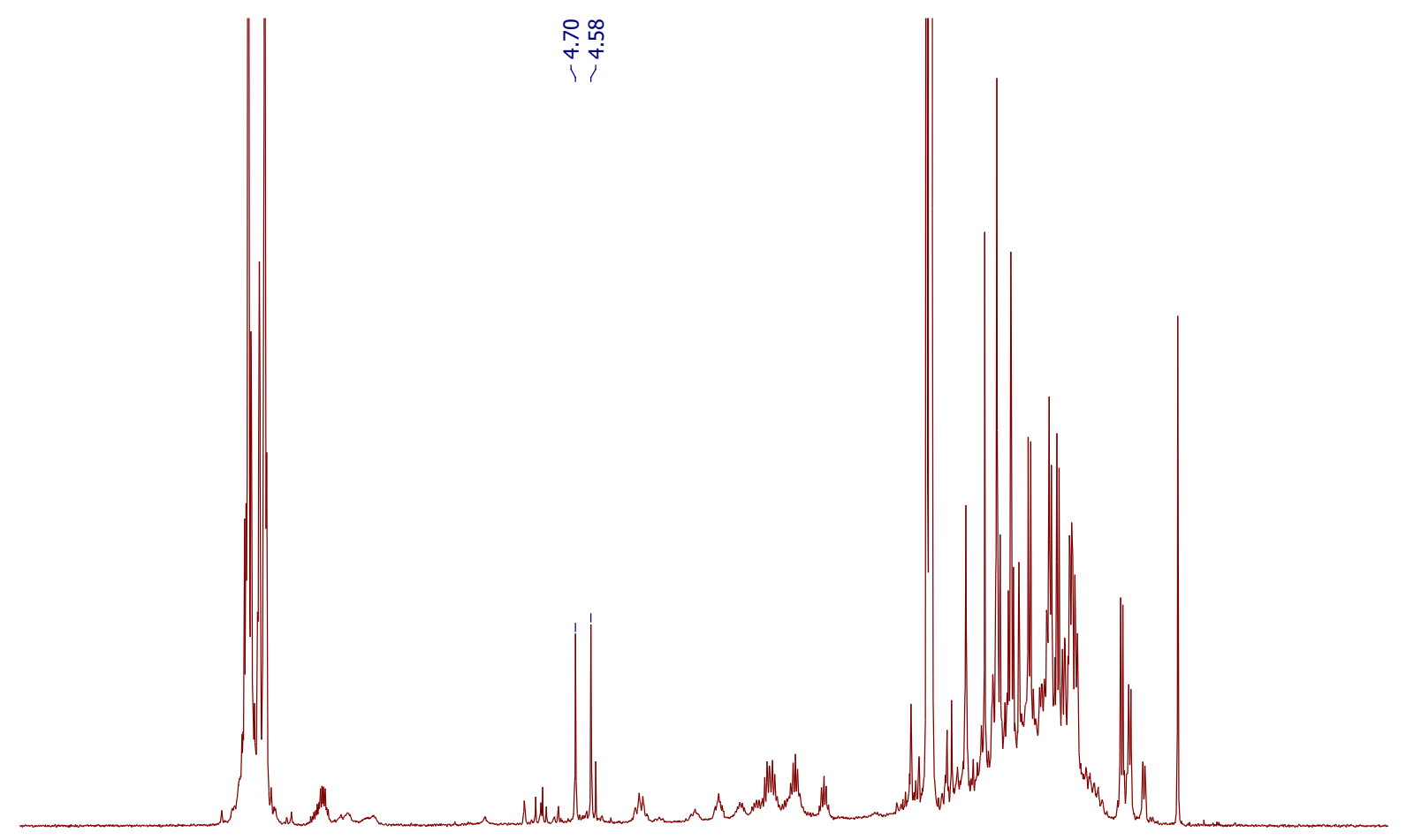

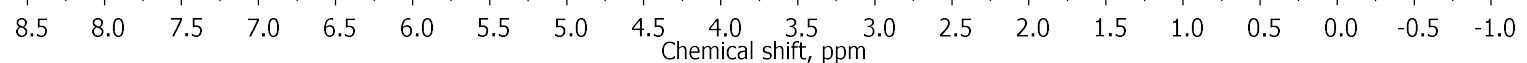

Figure S28. ${ }^{1} \mathrm{H}$ NMR spectrum $(400 \mathrm{MHz})$ of the final reaction mixture from the synthesis of $4 \mathrm{c}$ in toluene- $d_{8}$. Marked are the characteristic peaks of $4 \mathrm{c}(\mathrm{CH}$ methine). 2 is fully consumed. 


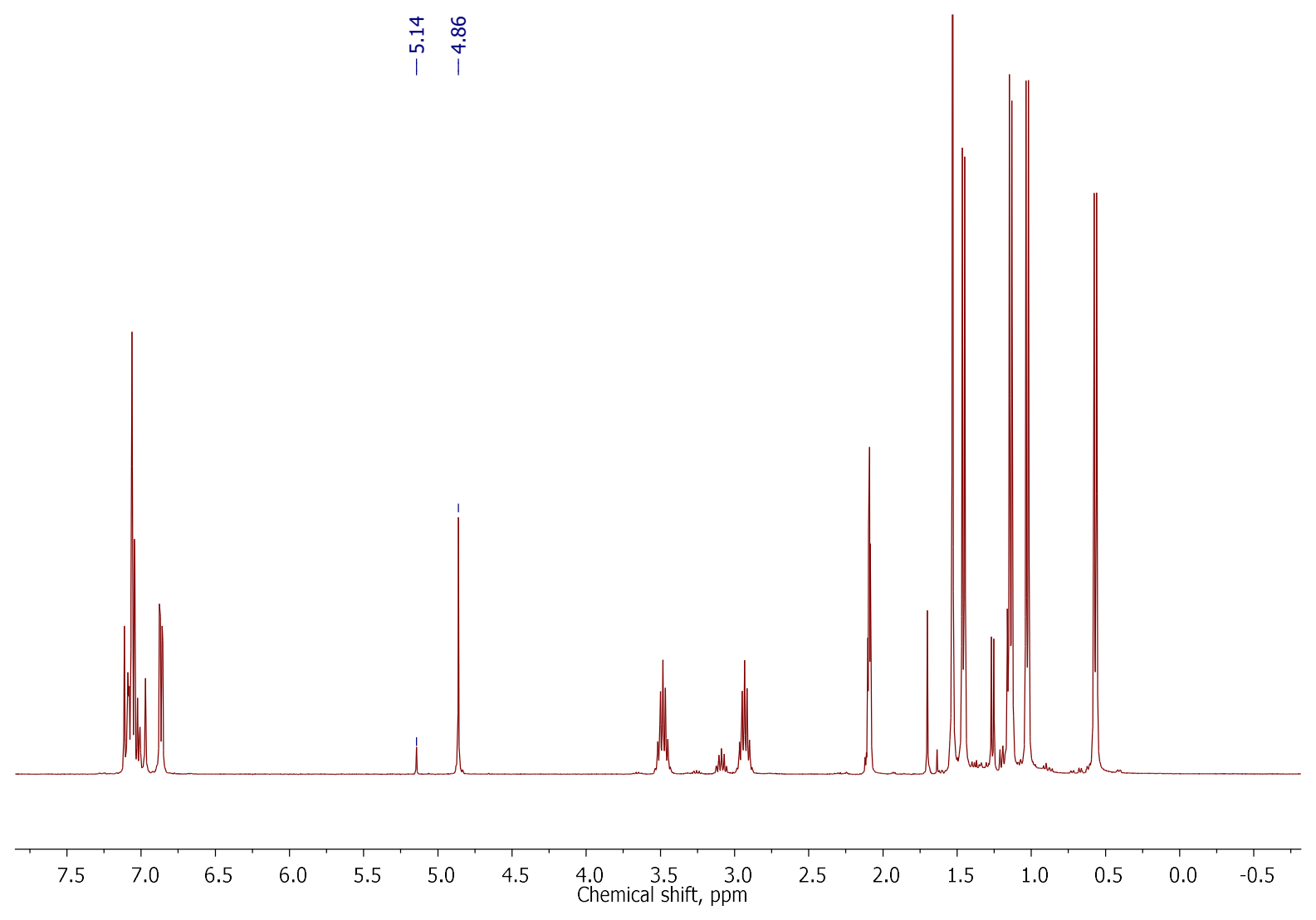

Figure S29. ${ }^{1} \mathrm{H}$ NMR spectrum $(400 \mathrm{MHz})$ of the final reaction mixture from the synthesis of $\mathbf{5 a}$ in toluene- $\mathrm{d}_{8}$. Marked are the characteristic peaks of $5 \mathbf{a}(4.86 \mathrm{ppm}, \mathrm{CH}$ methine) and of unreacted 3 (5.14 ppm, $\mathrm{CH}$ methine). 


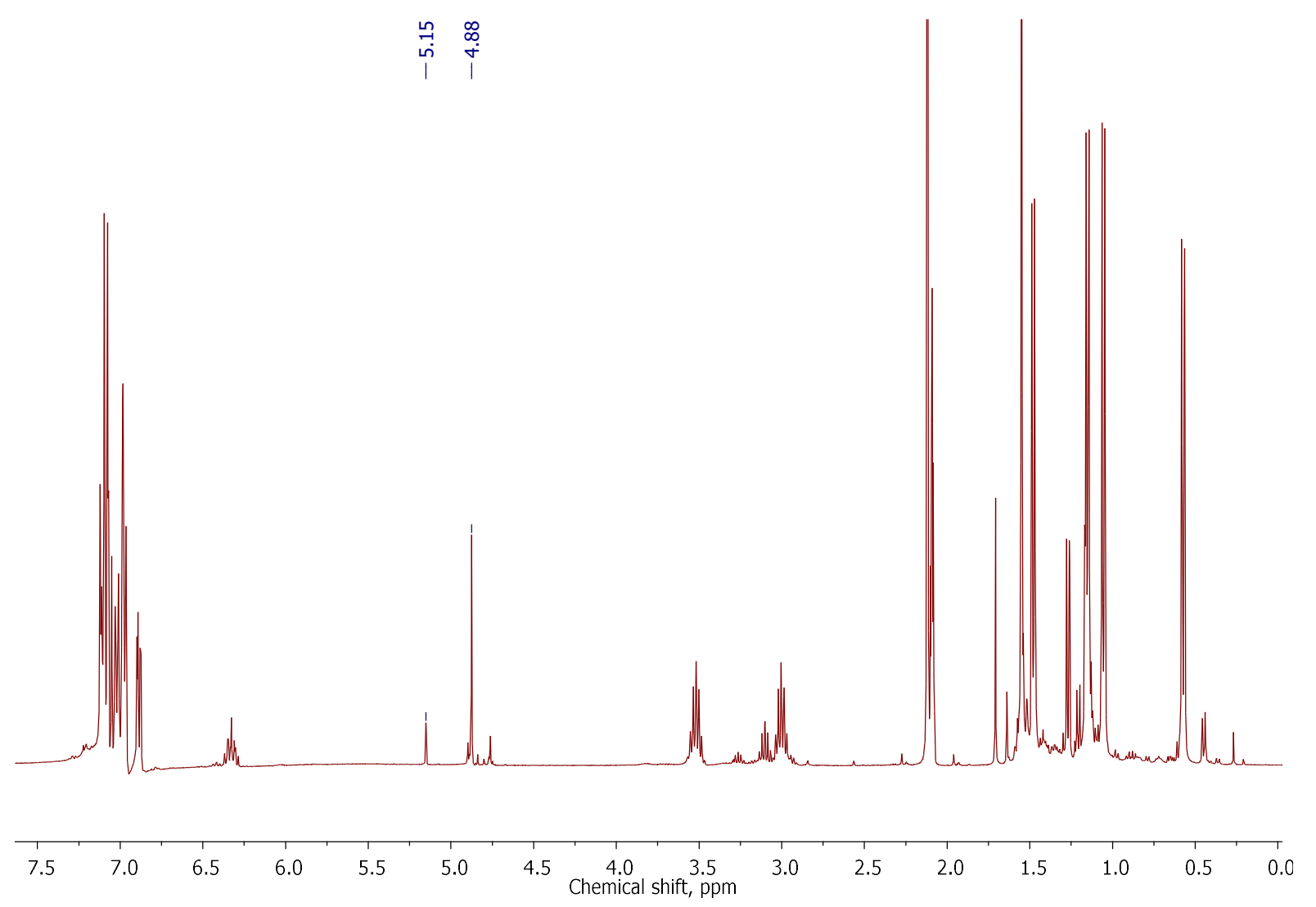

Figure S30. ${ }^{1} \mathrm{H}$ NMR spectrum $(400 \mathrm{MHz})$ of the final reaction mixture from the synthesis of $\mathbf{5 b}$ in toluene- $\mathrm{d}_{8}$. Marked are the characteristic peaks of $5 \mathbf{b}(4.88 \mathrm{ppm}, \mathrm{CH}$ methine) and of unreacted 3 (5.15 ppm, CH methine).

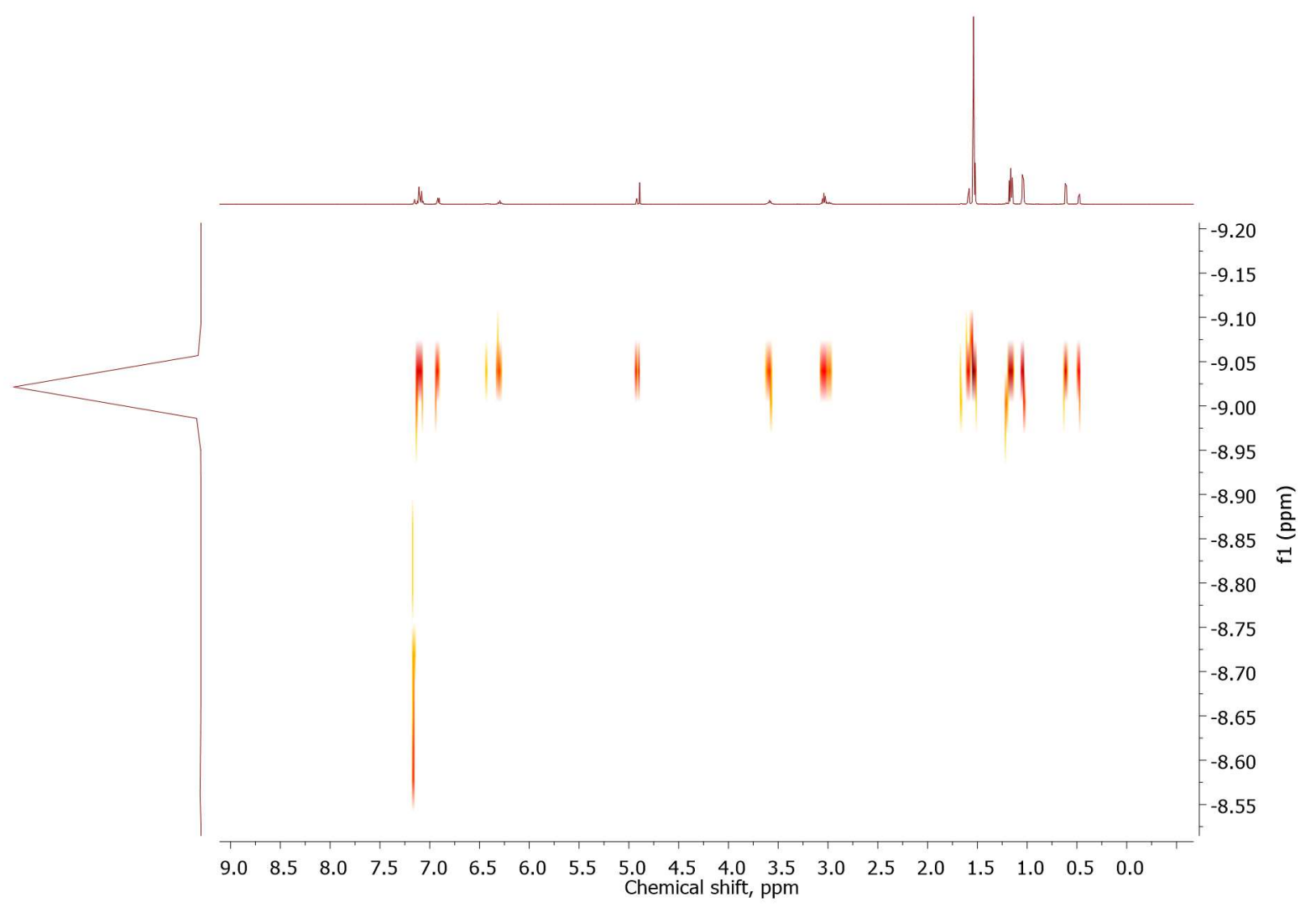

Figure S31. ${ }^{1} \mathrm{H}$ DOSY NMR $(600 \mathrm{MHz})$ spectrum of $\mathbf{5 b}$ in $\mathrm{C}_{6} \mathrm{D}_{6}$. 


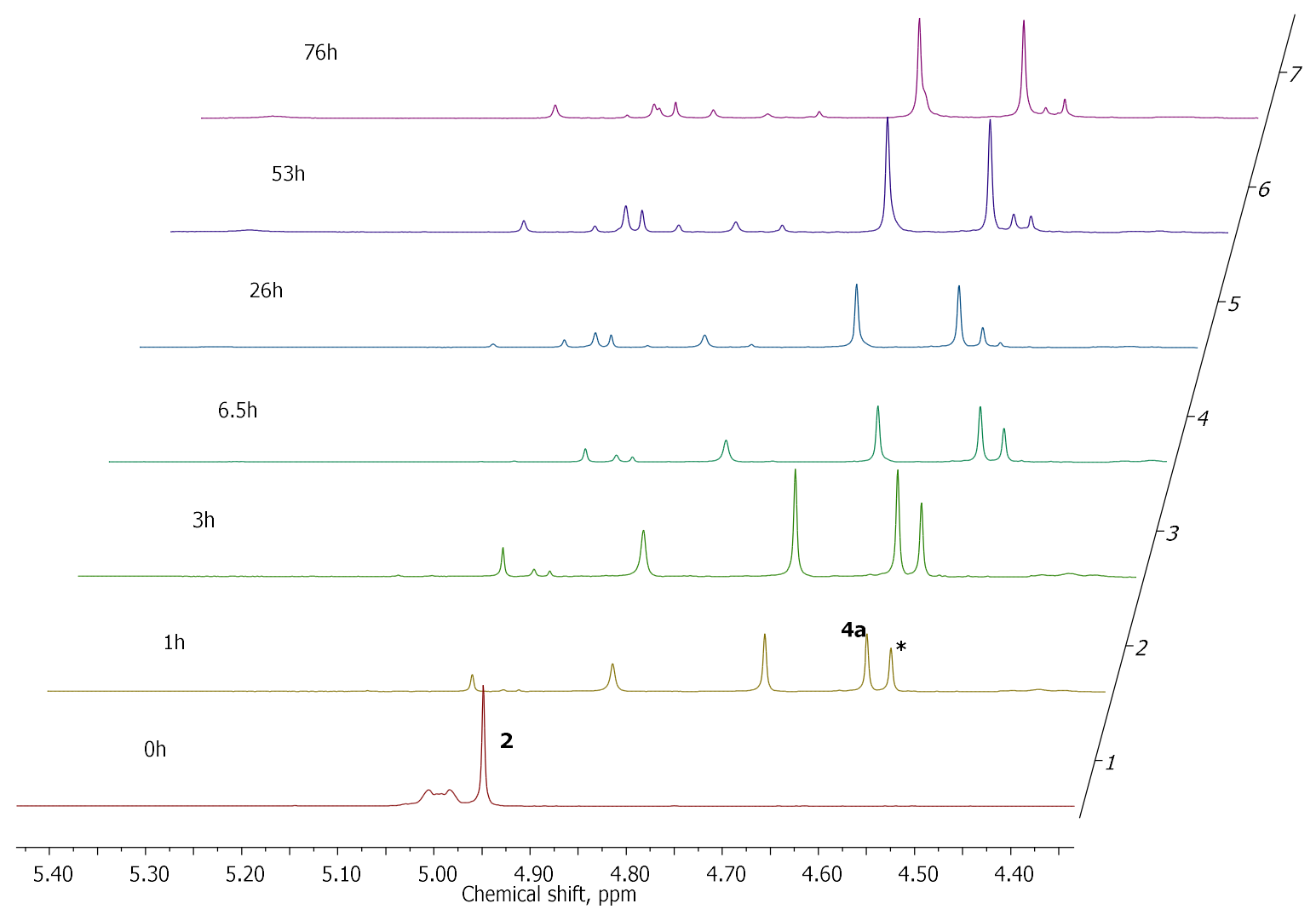

Figure S32. Series of ${ }^{1} \mathrm{H}$ NMR spectra taken at different time from the reaction mixture of 2 and $\mathrm{C}_{6} \mathrm{~F}_{6}$; $20 \mathrm{mg}(0.027 \mathrm{mmol})$ of 2 were dissolved in $0.5 \mathrm{~mL}$ of toluene- $\mathrm{d}_{8}$, treated with $6 \mu \mathrm{L}(0.036 \mathrm{mmol})$ of $\mathrm{Ph}_{2} \mathrm{CH}_{2}$ (as a standard) and $6 \mu \mathrm{L}(0.052 \mathrm{mmol})$ of $\mathrm{C}_{6} \mathrm{~F}_{6}$ and heated to $105^{\circ} \mathrm{C}$. 


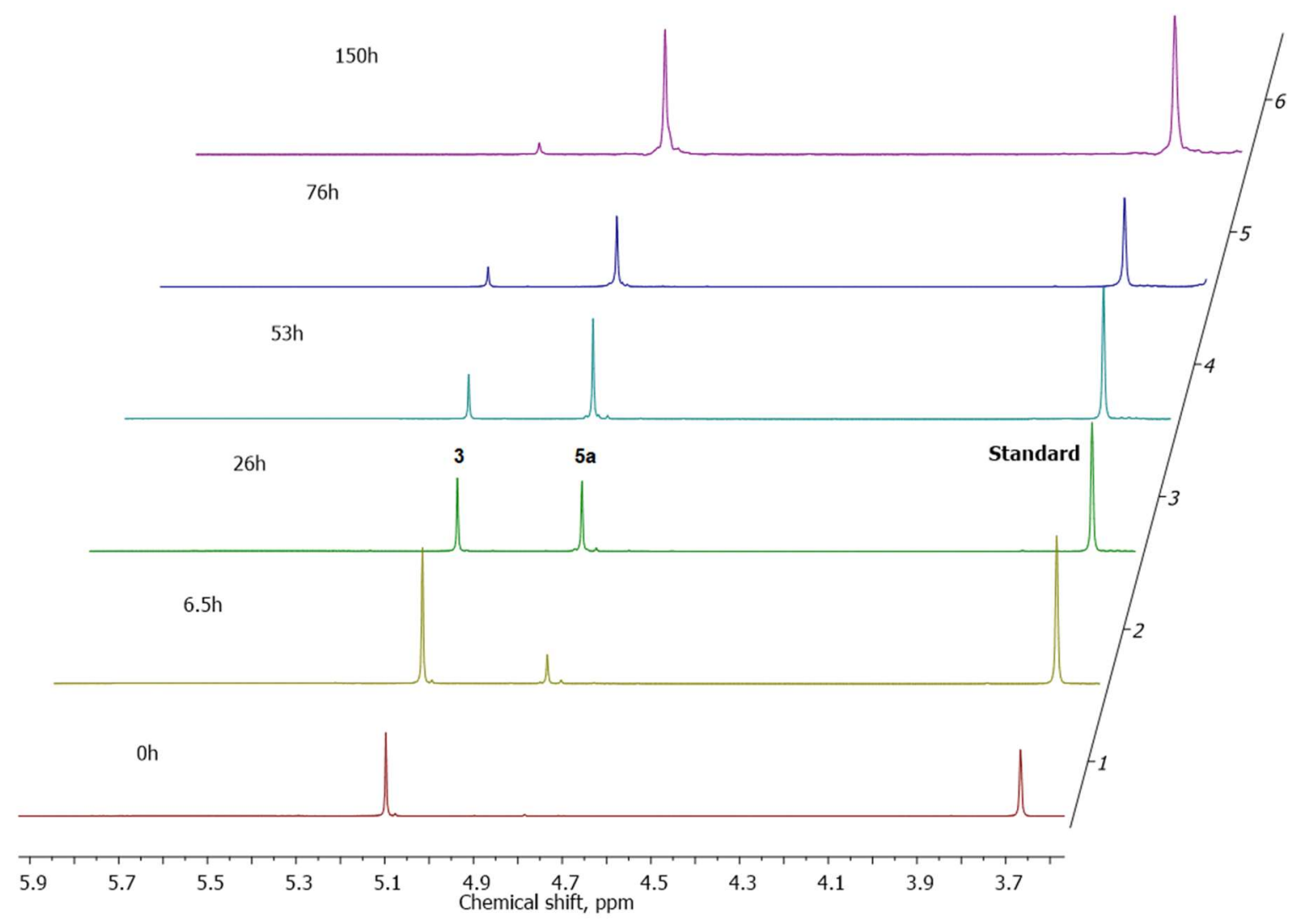

Figure S33. Series of ${ }^{1} \mathrm{H}$ NMR spectra taken at different time from the reaction mixture of 3 and $\mathrm{C}_{6} \mathrm{~F}_{6}$; $26 \mathrm{mg}(0.053 \mathrm{mmol})$ of 3 were dissolved in $0.5 \mathrm{~mL}$ of toluene- $\mathrm{d}_{8}$, treated with $6 \mu \mathrm{L}(0.036 \mathrm{mmol})$ of $\mathrm{Ph}_{2} \mathrm{CH}_{2}$ (as a standard) and $6 \mu \mathrm{L}(0.052 \mathrm{mmol})$ of $\mathrm{C}_{6} \mathrm{~F}_{6}$ and heated to $105^{\circ} \mathrm{C}$.

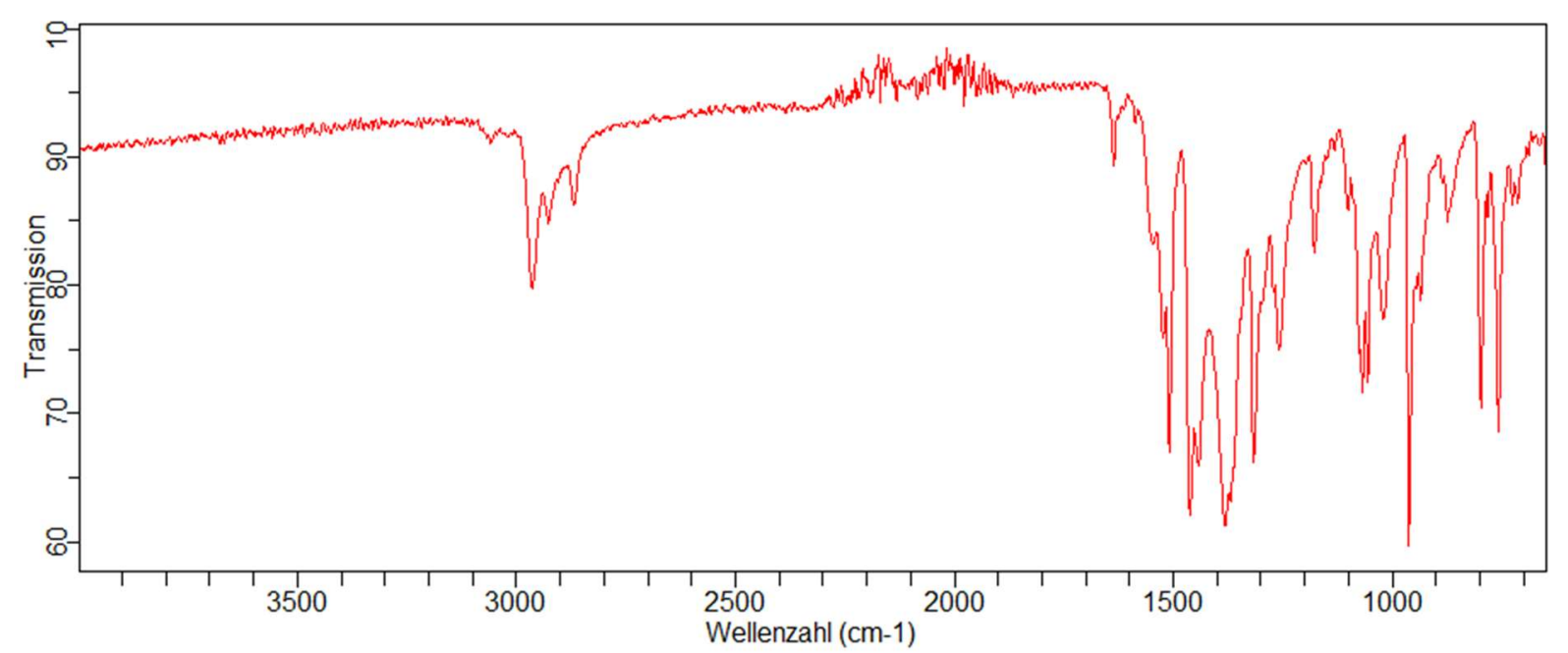

Figure S34. ATR-IR (diamond) spectrum of 5a (crude sample obtained from full-scale synthesis) indicating absence of characteristic bands of $\mathrm{GaOH}$ (around $3700 \mathrm{~cm}^{-1}$ ) and $\mathrm{GaH}$ (around $1800 \mathrm{~cm}^{-1}$ ). 


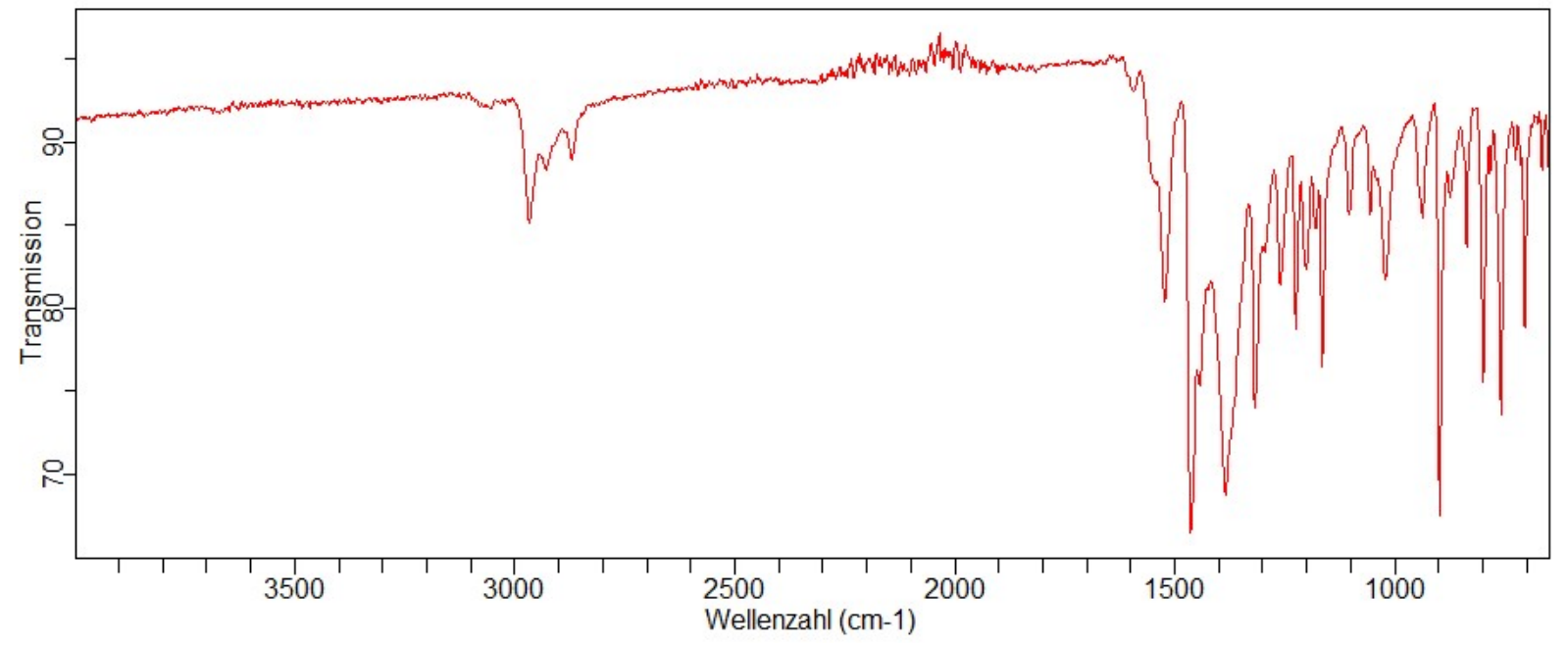

Figure S35. ATR-IR (diamond) spectrum of $\mathbf{5 b}$ (crude sample obtained from full-scale synthesis) indicating absence of characteristic bands of $\mathrm{GaOH}$ (around $3700 \mathrm{~cm}^{-1}$ ) and $\mathrm{GaH}$ (around $1800 \mathrm{~cm}^{-1}$ ). 


\section{XYZ-coordinates}

2

$\mathrm{Ga}$

$\mathrm{Ga}$

$\mathrm{N}$

$\mathrm{N}$

$\mathrm{N}$

N

C

C

C

$\mathrm{H}$

$\mathrm{C}$

C

$\mathrm{H}$

$\mathrm{H}$

$\mathrm{H}$

C

C

$\mathrm{H}$

$\mathrm{H}$

H

C

$\mathrm{H}$

$\mathrm{H}$

$\mathrm{H}$

C $\begin{array}{lll}-1.884868 & 0.985756 & 0.749740\end{array}$ $2.020124 \quad 1.140603-0.701900$ $-2.530936-0.549843-0.388694$ $-1.0273271 .814294-0.919940$ $\begin{array}{llll}1.079229 & 1.880030 & 0.960728\end{array}$ $2.575405-0.4676130 .382666$ $-3.451722-1.3886780 .316441$ $-2.994461-2.2901801 .290940$ $-3.941019-3.0413311 .997525$ $-3.602019-3.7433192 .754876$ $-5.297394-2.9069991 .751848$ $-6.014981-3.5036892 .306622$ $-5.738441-1.9930510 .798902$ $-6.803688-1.8799190 .624434$ $-4.835647-1.2127030 .081546$ $-1.520226-2.4843981 .611803$ $-0.923114-1.8766210 .919409$ $-1.104080-3.9495661 .430604$ $-1.319373-4.3094770 .419734$ $-1.630751-4.5994002 .137729$ $-0.030644-4.0653061 .615753$ $-1.204820-2.0242983 .041136$ $-1.430374-0.9615873 .176880$ $-0.143450-2.1811663 .260853$ $-1.790922-2.5972323 .768866$ $-5.327008-0.124087-0.864275$ $-4.615741-0.032000-1.692208$ $-6.701950-0.405072-1.471578$ $-6.742679-1.394183-1.939574$ $\begin{array}{llll}-6.930128 & 0.345196 & -2.234637\end{array}$ $-7.495112-0.351962-0.718483$ $-5.3256651 .218885-0.120671$ $\begin{array}{lll}-4.333366 & 1.406089 & 0.299483\end{array}$ $\begin{array}{lll}-6.036804 & 1.196383 & 0.712065\end{array}$ $-5.5885302 .045678-0.788540$ $-2.271318-0.803336-1.670589$ $-1.6029960 .092317-2.506276$ $-1.503550-0.200526-3.543379$ $-1.0742971 .352396-2.164961$ $-0.4921962 .149506-3.314408$ $0.603757 \quad 2.150783-3.250436$ $-0.8343113 .187560-3.304810$ $-0.7777871 .701989-4.266905$ $-2.751964-2.103815-2.273861$ $-2.501574-2.941937-1.615782$ $-2.304907-2.268697-3.255965$ $-3.842392-2.104649-2.381816$ $\begin{array}{llll}1.208354 & 4.383632 & 0.781559\end{array}$ $\begin{array}{llll}1.945387 & 4.352920 & -0.033858\end{array}$ $0.3288125 .632956 \quad 0.662817$ $-0.3348395 .6812881 .537239$ $0.947648 \quad 6.536023 \quad 0.684587$ $-0.5227785 .605178-0.611140$ $-1.2021256 .463739-0.630937$ $0.1356855 .700643-1.485589$ $-1.3148914 .299075-0.732926$ $-1.8831274 .273056-1.669229$ $\begin{array}{lll}-2.0483574 .215715 & 0.082336\end{array}$ $\begin{array}{llll}-0.362568 & 3.097688 & -0.667977\end{array}$ $0.4216533 .250109-1.419373$ $\begin{array}{llll}0.339242 & 3.121483 & 0.714576\end{array}$ $-0.4533403 .2200861 .466080$ $\begin{array}{llll}1.104278 & 1.385321 & 2.193570\end{array}$ $\begin{array}{lll}1.616553 & 0.111613 & 2.505462\end{array}$ $1.483355-0.2191883 .527550$ $2.266021-0.7719231 .643166$ $2.654009-2.1279012 .187716$ $3.741652-2.2512332 .210033$ $2.262688-2.9153061 .533880$ $2.263828-2.2722883 .196319$ $\begin{array}{llll}0.498857 & 2.147485 & 3.353708\end{array}$ $-0.5962222 .088597 \quad 3.302146$ 0.7869473 .2015243 .345202 $\begin{array}{llll}0.819948 & 1.709255 & 4.299389\end{array}$ $3.449714-1.335380-0.345492$ $2.948803-2.157664-1.366360$ $3.849577-2.964604-2.071926$
$3.474570-3.614837-2.858114$ $5.204957-2.949338-1.788892$ $5.886330-3.587169-2.343550$ $5.693551-2.100114-0.799282$ $6.759826-2.076947-0.598306$ $4.837403-1.274289-0.076351$ $1.474381-2.215079-1.734517$ $0.914200-1.532412-1.082570$ $0.908830-3.624133-1.524256$ $1.078084-3.976620-0.502377$ $1.374263-4.342730-2.208139$ $-0.169885-3.632004-1.716271$ $1.255366-1.766483-3.184350$ $0.197777-1.860070-3.449887$ $1.835277-2.386552-3.876884$ $1.555268-0.722287-3.323670$ $5.384153-0.2620520 .922008$ $4.670415-0.1657461 .747694$ $\begin{array}{llll}5.473666 & 1.110989 & 0.241158\end{array}$ $\begin{array}{llll}4.500428 & 1.382099 & -0.177684\end{array}$ $6.1914471 .081243-0.585637$ $\begin{array}{llll}5.782175 & 1.887448 & 0.948691\end{array}$ $6.733019-0.6533381 .526326$ $6.708654-1.6620341 .951743$ $\begin{array}{llll}6.999375 & 0.048251 & 2.322567\end{array}$ $7.534905-0.6171670 .781432$ $1.776384 \quad 4.393120 \quad 1.718298$

$-1.523698-0.8004371 .082031$ $1.061648-0.4894340 .092293$ $\begin{array}{llll}-2.438720 & 0.220430 & -0.447254\end{array}$ $-0.971691-2.214802-0.378381$ $1.913215-2.295935-0.411644$ $2.8147330 .409549-0.317684$ $\begin{array}{llll}-3.386446 & 1.177428 & 0.028709\end{array}$ $\begin{array}{lll}-2.9596472 .430807 & 0.500217\end{array}$ $-3.9073883 .2877031 .064570$ $\begin{array}{lll}-3.591029 & 4.256800 & 1.441509\end{array}$ $-5.2425012 .9187361 .159398$ $-5.9653273 .5967671 .603190$ $\begin{array}{lll}-5.650093 & 1.674763 & 0.690367\end{array}$ $\begin{array}{lll}-6.693991 & 1.386231 & 0.777482\end{array}$ $\begin{array}{lll}-4.735891 & 0.784065 & 0.128133\end{array}$ $\begin{array}{lll}-1.5018012 .849762 & 0.418021\end{array}$ $\begin{array}{llll}-0.978193 & 2.131176 & -0.222533\end{array}$ $-0.8196622 .8010701 .791749$ $0.2610742 .940301 \quad 1.675444$ $-0.9944791 .8413012 .289682$ $-1.2074763 .5941652 .442884$ $-1.3410354 .239775-0.205632$ $-0.2782824 .484466-0.300561$ $-1.8018625 .0130640 .418566$ $-1.8026934 .293738-1.198115$ $-5.171144-0.610251-0.295299$ $-4.410584-1.011692-0.973184$ $-5.209650-1.5302170 .933169$ $-5.467861-2.5555290 .645852$ $-5.953991-1.1737751 .654543$ $-4.234524-1.5393351 .432563$ $-6.513099-0.622555-1.031439$ $-6.5138970 .073326-1.876751$ $-7.340905-0.348846-0.368683$ $-6.722317-1.627074-1.413000$ $-2.1731990 .135012-1.741604$ $-1.451021-0.920568-2.327229$ $-1.353163-0.890913-3.405816$ $-0.984914-2.091158-1.689248$ $-0.410605-3.147278-2.610633$ $-0.563282-4.156893-2.226368$ $0.666035-2.978585-2.734360$ $-0.872728-3.081736-3.597472$ $-2.7341941 .188775-2.670337$ $-2.3734631 .050659-3.690799$ $\begin{array}{llll}-2.453380 & 2.189149 & -2.322448\end{array}$ $-3.8291381 .148576-2.670579$ $-0.270089-3.2541250 .358564$ $-0.212473-2.8451121 .382974$ $-1.020912-4.5789080 .529928$ $-2.068170-4.3554330 .760538$ $-1.006845-5.160674-0.401491$ $-0.373677-5.3809791 .664276$ 
$-0.536873-4.8388202 .604943$ $-0.859354-6.3565941 .769854$ $1.132531-5.5606721 .443529$ $1.296066-6.2245000 .582332$ $1.584850-6.0541572 .310181$ $1.837263-4.2265261 .171561$ $1.752286-3.5574142 .039115$ $2.907888-4.3852741 .007950$ $1.195029-3.523008-0.038380$ $1.201766-4.226250-0.879177$ $3.048130-2.439967-1.097482$ $3.971666-1.412740-1.344797$ $4.867703-1.689256-1.883896$ $3.890710-0.086217-0.914527$ $\begin{array}{llll}5.086744 & 0.805711 & -1.145043\end{array}$ $\begin{array}{llll}5.411133 & 1.250415 & -0.198733\end{array}$ $\begin{array}{llll}4.831375 & 1.635276 & -1.812397\end{array}$ $\begin{array}{llll}5.915313 & 0.245137 & -1.579158\end{array}$ $3.427642-3.788849-1.686225$ $3.502686-4.575354-0.932771$ $4.384973-3.719813-2.202468$ $2.674742-4.110721-2.414690$ $\begin{array}{llll}2.792150 & 1.782607 & 0.083012\end{array}$ $\begin{array}{llll}2.456061 & 2.773897 & -0.859410\end{array}$ $2.373924 \quad 4.096149-0.421040$ $2.1194274 .878772-1.131178$ $\begin{array}{llll}2.606905 & 4.430023 & 0.909384\end{array}$ $2.5339965 .464582 \quad 1.230418$ $2.923343 \quad 3.4360911 .826164$ $\begin{array}{llll}3.090140 & 3.699359 & 2.867521\end{array}$ $\begin{array}{llll}3.012036 & 2.097379 & 1.435984\end{array}$ $\begin{array}{llll}2.189979 & 2.430935 & -2.319651\end{array}$ $2.5588351 .417154-2.505976$ $0.6871412 .420914-2.629146$ $0.1627641 .667224-2.031628$ $\begin{array}{llll}0.518171 & 2.188110 & -3.686708\end{array}$ $0.241238 \quad 3.399655-2.417551$ $\begin{array}{llll}2.922029 & 3.378496 & -3.277546\end{array}$ $\begin{array}{llll}3.988162 & 3.454647 & -3.039658\end{array}$ $\begin{array}{lllll}2.500118 & 4.388301 & -3.241404\end{array}$ $2.8238573 .021105-4.307295$ $\begin{array}{lll}3.332729 & 1.027736 & 2.469504\end{array}$ $\begin{array}{llll}3.457097 & 0.070965 & 1.950942\end{array}$ $\begin{array}{llll}2.192481 & 0.869961 & 3.485396\end{array}$ $\begin{array}{llll}1.246767 & 0.595352 & 3.005616\end{array}$ $2.030183 \quad 1.805664 \quad 4.032813$ $2.442398 \quad 0.091414 \quad 4.214053$ $\begin{array}{llll}4.653288 & 1.326396 & 3.190754\end{array}$ $\begin{array}{llll}4.904044 & 0.509985 & 3.875387\end{array}$ $\begin{array}{llll}4.584109 & 2.245207 & 3.782837\end{array}$ 5.4790141 .4460152 .482016

$-1.2456900 .0689211 .903954$ $1.245523-0.0699521 .903877$ $-0.000053-0.0007292 .539868$ $-0.000007-0.0009643 .621889$ $2.710192-0.0809930 .026576$ $3.4376921 .117045-0.120908$ $3.206135-1.302445-0.459386$ $\begin{array}{llll}4.683444 & 1.061402 & -0.743941\end{array}$ $4.457758-1.309207-1.081121$ $5.194465-0.141957-1.220849$ $\begin{array}{llll}5.263113 & 1.971396 & -0.866268\end{array}$ $4.855973-2.245091-1.465110$ $6.164963-0.164873-1.706935$ $-2.7102650 .0810220 .026641$ $-3.437877-1.116956-0.121000$ $-3.2061611 .302569-0.459133$ $-4.683750-1.061096-0.743762$ $-4.4578871 .309521-1.080676$ $-5.1947570 .142386-1.220391$ $-5.263563-1.970997-0.866051$ $-4.8560542 .245486-1.464517$ $-6.1653750 .165485-1.706229$ $-2.4170232 .597769-0.341087$ $-3.2047363 .6670560 .425987$ $-2.0077623 .121203-1.723802$ $\begin{array}{lll}-1.502382 & 2.396179 & 0.227999\end{array}$ $-3.5012333 .3130951 .418427$ $-2.5979404 .5696140 .551354$ $-4.1149423 .951078-0.113084$
$\mathrm{H}$

$1,2,3,4-\mathrm{C}_{6} \mathrm{H}_{2} \mathrm{~F}_{4}$ (c)

$\mathrm{C}$
$\mathrm{C}$
$\mathrm{C}$
$\mathrm{C}$
$\mathrm{C}$
$\mathrm{C}$
$\mathrm{H}$

$-1.4196582 .373638-2.265635$ $-2.8913623 .360059-2.326224$ $-1.4076304 .032315-1.625762$ $-2.847967-2.4439050 .335807$ $-2.028196-3.074724-0.798576$ $-3.901617-3.4280470 .849409$ $-2.153965-2.2417981 .159238$ $-1.223183-2.403306-1.116290$ $-1.581915-4.022338-0.477685$ $-2.663244-3.264889-1.670578$ $-4.547263-2.9689951 .605179$ $-4.538760-3.7970770 .038898$ $-3.412171-4.2982571 .297313$ 2.8477732 .4438290 .336314 $\begin{array}{llll}2.028686 & 3.075464 & -0.798098\end{array}$ $\begin{array}{llll}3.901398 & 3.427422 & 0.851075\end{array}$ $2.153332 \quad 2.241359 \quad 1.159289$ $1.2236222 .404402-1.116446$ $\begin{array}{llll}1.582566 & 4.023061 & -0.476947\end{array}$ $2.6641493 .265823-1.669758$ $\begin{array}{llll}4.546700 & 2.967663 & 1.606716\end{array}$ $\begin{array}{llll}4.538901 & 3.796975 & 0.041088\end{array}$ $\begin{array}{llll}3.411919 & 4.297358 & 1.299473\end{array}$ $2.417078-2.597683-0.341327$ $3.204889-3.6669060 .425723$ $2.007813-3.121119-1.724052$ $1.502430-2.3961710 .227766$ $3.501246-3.3129801 .418224$ $2.598226-4.5695700 .550981$ $4.115186-3.950758-0.113280$ $1.420064-2.373405-2.266075$ $2.891417-3.360374-2.326310$ $1.407313-4.031987-1.626008$ $-2.4728050 .1822232 .778883$ $-3.132192-0.6805922 .636565$ $-2.1993100 .248503 \quad 3.832535$ $-3.0533321 .0682842 .502527$ $2.472702-0.1838162 .778642$ $2.199313-0.2511223 .832254$ $3.053501-1.0694192 .501433$ $\begin{array}{llll}3.131833 & 0.679334 & 2.637038\end{array}$ $\begin{array}{lll}-1.393398 & 0.035636 & 0.584042\end{array}$ $1.393290-0.0357350 .583950$ $\begin{array}{llll}0.000209 & 0.000476 & -0.913059\end{array}$

$1.2141090 .675583-0.000002$ $1.192034-0.7136170 .000022$ $-0.021998-1.389173-0.000003$ $-1.214140-0.6755240 .000022$ $\begin{array}{llll}-1.192068 & 0.713561 & -0.000000\end{array}$ 0.0220641 .3891720 .000021 $\begin{array}{llll}2.374218 & 1.320712 & -0.000014\end{array}$ $0.0428542 .716385-0.000001$ $-2.3309041 .395470-0.000013$ $-2.374122-1.3208820 .000001$ $-0.043048-2.716380-0.000015$ $2.331002-1.3953070 .000002$

$1.191662-0.9662230 .000006$ $\begin{array}{llll}1.205209 & 0.422996 & 0.000002\end{array}$ $\begin{array}{lll}-0.000003 & 1.114049 & 0.000005\end{array}$ $\begin{array}{lll}-1.205240 & 0.4229250 .000002\end{array}$ $-1.191658-0.9662360 .000007$ $0.000037-1.6740970 .000003$ $0.000005-2.7568670 .000003$ $-2.356728-1.610988-0.000005$ $-2.3537061 .092363-0.000004$ $-0.0000992 .4411510 .000000$ $2.353724 \quad 1.092350-0.000004$ $2.356805-1.610833-0.000005$

$0.696988-1.729930-0.000002$ $-0.696990-1.7299300 .000002$ $-1.379795-0.5263000 .000000$ $-0.6935000 .684155-0.000001$ 0.6935010 .6841550 .000002 $1.379794-0.526301-0.000003$ $1.264136-2.653053-0.000006$ 

$2.713663-0.4932080 .000001$ $\begin{array}{llll}1.356281 & 1.836040 & 0.000001\end{array}$ $-1.3562791 .836042-0.000001$ $-2.713664-0.4932060 .000000$

$4 a$

$\mathrm{Ga}$

$\mathrm{F}$

$\mathrm{H}$

C

$\mathrm{H}$
$1.317191-0.6100830 .093085$ $-1.014641-0.210068-0.288653$ $1.716032-1.8731801 .325180$ $-0.824785-0.5863922 .885001$ $-2.629961-1.5057504 .659978$ $-4.020383-0.993411-0.563044$ $\begin{array}{llll}2.928820 & 0.464206 & 0.017823\end{array}$ $1.700667-1.537384-1.629392$ $-1.157568-1.702108-1.604950$ $-1.7448041 .138075-1.474526$ $\begin{array}{llll}3.431593 & 0.841823 & 1.305233\end{array}$ $\begin{array}{llll}4.411266 & 0.036044 & 1.918419\end{array}$ $\begin{array}{llll}4.826910 & 0.373814 & 3.206590\end{array}$ $5.585240-0.2330673 .694669$ $4.266923 \quad 1.4479143 .887954$ $4.596264 \quad 1.685338 \quad 4.894900$ 3.2760232 .2072323 .281411 $2.827070 \quad 3.037417 \quad 3.820754$ 2.8391241 .9187521 .986996 $4.995906-1.1984631 .247125$ $4.551929-1.3030950 .250705$ $6.517587-1.0858481 .080683$ $6.906057-1.9639060 .554508$ $7.014423-1.0381882 .055918$ $6.811754-0.1950710 .516918$ $4.650690-2.4656412 .041761$ $5.031126-3.3493681 .516749$ $3.570293-2.5590672 .160842$ $5.118640-2.4413823 .032655$ $1.7618092 .779401 \quad 1.349046$ $\begin{array}{llll}1.596881 & 2.420740 & 0.327055\end{array}$ $2.207301 \quad 4.244471 \quad 1.267964$ $\begin{array}{llll}1.441300 & 4.845569 & 0.767995\end{array}$ $\begin{array}{llll}3.149247 & 4.349933 & 0.718187\end{array}$ $2.360864 \quad 4.662266 \quad 2.268936$ 0.4226192 .6748372 .091664 -0.360024 3.1848871 .517176 0.4875923 .1502503 .077711 0.1217891 .6330652 .251163 $\begin{array}{llll}3.597640 & 0.720297 & -1.100091\end{array}$ $\begin{array}{llll}3.307737 & 0.116061 & -2.331211\end{array}$ $\begin{array}{llll}3.885443 & 0.458830 & -3.180104\end{array}$ $2.485843-1.014615-2.549827$ $2.566460-1.645412-3.923800$ $3.235065-1.076733-4.570134$ $1.575834-1.673654-4.390521$ $2.937423-2.673604-3.867159$ $4.7489541 .691768-1.033960$ $\begin{array}{llll}5.110828 & 1.940596 & -2.032574\end{array}$ $\begin{array}{llll}5.574222 & 1.263232 & -0.457414\end{array}$ $\begin{array}{llll}4.441517 & 2.607494 & -0.518720\end{array}$ $1.058606-2.841113-1.803425$ $0.929937-3.069791-2.868526$ $1.940367-3.922450-1.153616$ $2.900428-3.955213-1.683149$ $2.150060-3.595448-0.127808$ $1.270399-5.296388-1.137466$ $1.132533-5.659857-2.166002$ $1.921235-6.016887-0.631655$ $-0.092235-5.224135-0.444981$ $0.044581-4.9054160 .596644$ $-0.567347-6.210197-0.424056$ $-0.993940-4.226661-1.176134$ $-1.140140-4.593440-2.195408$ $-1.981046-4.154588-0.704410$ $-0.344188-2.838595-1.153503$ $-0.168901-2.657006-0.080648$ $-1.844915-1.624087-2.722144$ $-2.541841-0.452902-3.112916$ $-3.155404-0.544889-3.999175$ $-2.4836140 .817431-2.543314$ $-3.2313251 .921026-3.250716$ $-3.7556011 .536956-4.125756$ $-2.5415682 .711255-3.564262$ $-3.9578852 .383436-2.575447$
$-1.942317-2.775905-3.701884$ $-2.304580-2.416425-4.665496$ $-2.643904-3.531370-3.335948$ $-0.977030-3.261077-3.858684$ $-1.5839692 .515967-1.117553$ $-0.4287133 .201164-1.558570$ $-0.3265494 .559119-1.247196$ $0.5332485 .125820-1.585828$ $-1.2989115 .210215-0.493204$ $-1.1900546 .266687-0.267031$ $-2.3870034 .500660-0.010065$ $-3.1239875 .0003140 .612917$ $-2.5465513 .144939-0.308104$ $\begin{array}{llll}0.694156 & 2.485737 & -2.312779\end{array}$ $0.9603901 .590768-1.730568$ $0.3135151 .993647-3.716970$ $-0.4266571 .192405-3.694792$ $\begin{array}{llll}1.210148 & 1.596176 & -4.206714\end{array}$ $-0.0668692 .818487-4.331141$ $\begin{array}{llll}1.958878 & 3.340198 & -2.425379\end{array}$ $2.3033213 .703455-1.451164$ $\begin{array}{llll}1.793868 & 4.207312 & -3.075404\end{array}$ $\begin{array}{llll}2.758637 & 2.742672 & -2.870095\end{array}$ $\begin{array}{lll}-3.7003182 .375603 & 0.312460\end{array}$ $-3.7445881 .384174-0.147304$ $-3.4406152 .2019201 .817233$ $-4.1759621 .5196642 .260124$ $-3.5154563 .1676942 .330745$ $\begin{array}{lll}-2.442120 & 1.797927 & 2.008625\end{array}$ $\begin{array}{lll}-5.059992 & 3.048051 & 0.091105\end{array}$ $\begin{array}{lll}-5.8574862 .418532 & 0.498269\end{array}$ $-5.2675173 .213934-0.970631$ $\begin{array}{lll}-5.115534 & 4.017509 & 0.597381\end{array}$ $-2.351202-0.7785021 .090336$ $-2.040925-0.9314932 .430671$ $-2.955795-1.3825223 .374106$ $-4.239145-1.7139732 .957864$ $-4.592554-1.5811051 .620283$ $-3.637313-1.1267390 .722586$ $-5.828295-1.9000111 .231315$ $-5.132234-2.1546063 .840014$
$\mathrm{Ga}$
$1.0982510 .340834-0.097268$ $\begin{array}{lll}-1.279551 & 0.549966 & 0.120022\end{array}$ $-1.8644101 .6468021 .434889$ $\begin{array}{llll}4.051857 & 1.319959 & -0.031769\end{array}$ $\begin{array}{llll}5.647122 & 2.103511 & 1.982170\end{array}$ $2.216371 \quad 1.138247 \quad 5.069855$ $\begin{array}{lll}0.630086 & 0.340958 & 3.069150\end{array}$ $2.008511-0.816000-1.360047$ $1.2338171 .978722-1.229361$ $-1.5955751 .638283-1.520402$ $-2.803619-0.604936-0.201833$ $1.918430-2.232791-1.170302$ $0.850486-2.932168-1.777731$ $0.818881-4.320332-1.624915$ $0.028298-4.895667-2.093011$ $1.774043-4.992994-0.867908$ $1.721453-6.072913-0.766986$ $2.771115-4.278556-0.222761$ $3.491860-4.8004910 .400971$ $2.858823-2.890534-0.357472$ $-0.256892-2.204999-2.543347$ $-0.626465-1.396265-1.895134$ $-1.448599-3.114560-2.850962$ $-2.249016-2.520250-3.298549$ $-1.843712-3.600466-1.952284$ $-1.174983-3.895079-3.570346$ $0.197574-1.540214-3.851218$ $-0.682435-1.139094-4.367487$ $0.673425-2.270442-4.516514$ $0.883360-0.708259-3.683563$ $3.907476-2.1295540 .435900$ $3.917239-1.0905330 .094488$ $3.527026-2.1475551 .924843$ $4.179174-1.4784112 .498535$ $3.629101-3.1606222 .331642$ $2.492328-1.8278402 .078963$ $5.322922-2.6889010 .252129$ $5.619524-2.717543-0.801006$ $5.407500-3.7056270 .650188$ 
$\begin{array}{llll}0.583877 & 0.238554 & 3.166862\end{array}$ $\begin{array}{llll}2.008939 & 1.036590 & 5.275084\end{array}$ 3.9941321 .6991340 .279006 $-2.632800-0.788156-0.334501$ $-1.5721141 .623833-1.464088$ $1.1986242 .190415-1.012292$ $2.217962-0.514754-1.264932$ $-3.206203-1.4502250 .799121$ $-4.363330-0.9078051 .392657$ $-4.858992-1.5193522 .544291$ $-5.753410-1.1181463 .014060$ $-4.213141-2.6071593 .119670$ $-4.609066-3.0598234 .023640$ $-3.052779-3.1035122 .542087$ $-2.538545-3.9438993 .001467$ $-2.527738-2.5360361 .379250$ $\begin{array}{lll}-5.060796 & 0.331650 & 0.850405\end{array}$ $\begin{array}{llll}-4.533652 & 0.669284 & -0.048990\end{array}$ $\begin{array}{llll}-6.518154 & 0.039904 & 0.467033\end{array}$ $\begin{array}{llll}-6.981258 & 0.933243 & 0.035370\end{array}$ $-7.104328-0.2432071 .348230$ $-6.604754-0.772405-0.261312$ $-5.0104371 .4761341 .872279$ $-5.4676012 .3762641 .445330$ $-3.9780191 .6963542 .147572$ $-5.5737741 .2144612 .775461$ $-1.262819-3.1125640 .766199$ $-1.046304-2.560207-0.154804$ $-1.456179-4.5876830 .394728$ $-0.552926-4.977981-0.084882$ $-2.301994-4.724196-0.288394$ $-1.650934-5.1933811 .286560$ $-0.047317-2.9551581 .690109$ $0.864986-3.2407141 .152579$ $-0.142280-3.6026412 .570092$ $0.063429-1.9266742 .052015$ $-3.130875-0.931547-1.556619$ $-2.812217-0.084302-2.626789$ $-3.234495-0.347605-3.588191$ $-2.1624591 .170363-2.551229$ $-2.1970832 .019282-3.804417$ $\begin{array}{llll}-2.692590 & 1.487330 & -4.616668\end{array}$ $-1.1797812 .269381-4.124622$ $-2.7324792 .958441-3.633152$ $-4.114392-2.048830-1.798485$ $-4.318301-2.168509-2.863539$ $-5.056399-1.843478-1.281247$ $-3.726259-2.988911-1.393514$ $-1.1381343 .015901-1.338083$ $-0.9280843 .446417-2.325046$ $-2.2554353 .826279-0.656692$ $-3.1401153 .816416-1.305242$ $\begin{array}{lll}-2.5252563 .293683 & 0.263445\end{array}$ $\begin{array}{lll}-1.827965 & 5.256480 & -0.326797\end{array}$ $-1.6339815 .814716-1.254114$ $-2.6429415 .7739530 .189660$ $\begin{array}{lll}-0.5614915 .256418 & 0.531973\end{array}$ $-0.7631854 .7385111 .478683$ $-0.2620556 .280358 \quad 0.778123$ $\begin{array}{llll}0.571198 & 4.546527 & -0.213392\end{array}$ $0.771925 \quad 5.108042-1.129668$ $\begin{array}{llll}1.494986 & 4.531518 & 0.376844\end{array}$ $0.1618963 .098366-0.505182$ $-0.1028112 .7037550 .489070$ $2.0048572 .411469-2.025698$ $2.9194221 .439792-2.504439$ $3.6006011 .774428-3.275473$ $3.0045490 .091949-2.160805$ $3.987489-0.751785-2.935438$ $4.536611-0.146260-3.656580$ $3.466949-1.555782-3.465730$ $4.699753-1.229424-2.255712$ $2.0186503 .722545-2.785945$ $2.5380993 .597460-3.736532$ $2.5424254 .492200-2.211339$ $1.0102064 .085800-2.992667$ $2.243790-1.942727-1.159138$ $1.267107-2.693124-1.853335$ $1.351025-4.085629-1.780014$ $0.633871-4.697176-2.315537$ $2.329473-4.718820-1.018767$ $2.367503-5.803422-0.981226$ 
$3.232412-3.961948-0.289030$ $3.969494-4.4569480 .337622$ $3.203489-2.565924-0.341487$ $0.131104-2.018885-2.625729$ $-0.336300-1.289415-1.947528$ $0.575166-1.232954-3.868335$ $1.168332-0.352185-3.617722$ $-0.316557-0.884933-4.402704$ $1.148150-1.871142-4.551519$ $-0.958934-3.008819-3.043039$ $-1.344638-3.584699-2.194851$ $-0.585555-3.714250-3.794469$ $-1.791228-2.460326-3.490986$ $4.147098-1.7673000 .541649$ $\begin{array}{llll}4.076602 & -0.712210 & 0.263141\end{array}$ $3.711840-1.9113542 .008484$ $4.273539-1.2197112 .647335$ . $893491-2.9336612 .361041$ $2.646212-1.6983822 .132943$ $5.613287-2.1878990 .389115$ $6.250711-1.5403870 .999660$ $\begin{array}{llll}5.954825 & -2.119506 & -0.648533\end{array}$ $5.772654-3.2178300 .725659$ $\begin{array}{llll}2.235501 & 0.966728 & 1.642559\end{array}$ $\begin{array}{llll}1.772469 & 0.823815 & 2.937064\end{array}$ $2.513530 \quad 1.216847 \quad 4.048115$ $3.7638861 .786646 \quad 3.882338$ $4.3235742 .084615 \quad 4.761918$ $\begin{array}{llll}4.272937 & 1.957111 & 2.594654\end{array}$ $5.2494082 .395278 \quad 2.423340$ $3.488702 \quad 1.548799 \quad 1.528395$

$5 a$

C $\begin{array}{lll}-1.726570 & 0.0126882 .081675\end{array}$ $0.093601-1.7198132 .022582$ $-0.855048-0.9075782 .670961$ $-0.988014-1.0882403 .729690$ $1.319779-2.5081050 .089606$ $2.711221-2.3640520 .232517$ $0.761474-3.479926-0.766855$ $3.538348-3.253505-0.459394$ $1.630981-4.344731-1.430326$ $3.009044-4.236589-1.280836$ $4.616424-3.163042-0.356046$ $1.228492-5.107699-2.087805$ $3.668218-4.917443-1.810436$ $-2.7757841 .0627220 .197440$ $\begin{array}{lll}-2.7326332 .458709 & 0.052028\end{array}$ $-4.9141310 .983151-0.898008$ $-3.8117553 .093555-0.566404$ $-4.8981012 .366199-1.034034$ $-5.7583840 .416004-1.281489$ $-3.7939994 .173415-0.689118$ $-5.7288922 .875412-1.512628$ $\begin{array}{lll}-1.535820 & 3.275051 & 0.506306\end{array}$ $-1.9258324 .3735331 .502418$ $-0.8242853 .889743-0.708200$ $-0.8374052 .6048661 .017832$ $-2.4183043 .9608212 .388425$ $\begin{array}{lll}-1.0352324 .918810 & 1.831064\end{array}$ $-2.6099145 .0972131 .046729$ $-0.6673263 .150221-1.498801$ $-1.4227574 .704590-1.131295$ $0.1499674 .300695-0.418095$ $-3.894930-1.211746-0.191091$ $-3.862411-1.850152-1.584760$ $-5.124692-1.6968890 .587425$ $-3.006388-1.5553210 .351539$ $-2.951959-1.570503-2.121697$ $-3.900920-2.941597-1.492256$ $-4.729785-1.536740-2.176684$ $-5.176134-1.2573261 .588546$ $-6.050884-1.4396150 .062256$ $-5.096249-2.7860500 .695976$ $3.354262-1.2961111 .103568$ $4.220831-0.3493020 .260404$ $4.216298-1.9195312 .211438$ $2.563711-0.7009031 .574569$ $\begin{array}{llll}3.651493 & 0.088238 & -0.564387\end{array}$ $\begin{array}{llll}4.614695 & 0.460862 & 0.884225\end{array}$ $5.074387-0.885308-0.168603$ $\begin{array}{lll}-3.856763 & 0.305447 & -0.290415\end{array}$
$3.664847-2.6616472 .795107$ $5.089888-2.4229371 .783571$ $4.581495-1.1436172 .891824$ $-0.746301-3.630345-0.909166$ $-1.322865-4.4730810 .234674$ $-1.155785-4.196650-2.268465$ $-1.183928-2.631796-0.841712$ $-1.127610-4.0160051 .210682$ $-2.408889-4.5720530 .123297$ $-0.886704-5.4785150 .234409$ $-0.716166-3.610941-3.080861$ $-0.855485-5.243724-2.384388$ $-2.243173-4.160242-2.373930$ $-2.8182840 .6003652 .938655$ $\begin{array}{lll}-3.800385 & 0.268642 & 2.587829\end{array}$ $\begin{array}{llll}-2.696770 & 0.307219 & 3.981474\end{array}$

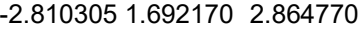
$0.755986-2.7789012 .869285$ $0.056960-3.1528153 .619643$ $1.128248-3.6129062 .272298$ $1.605303-2.3314103 .397471$ $\begin{array}{lll}-1.673834 & 0.367543 & 0.798340\end{array}$ $0.416696-1.6009110 .741434$ $-0.228842-0.144738-0.363350$ $1.1695321 .237462-0.527122$ $\begin{array}{llll}1.703871 & 1.732314 & -1.703902\end{array}$ $\begin{array}{lll}1.681104 & 1.760662 & 0.647271\end{array}$ $\begin{array}{llll}2.716446 & 2.685052 & -1.720303\end{array}$ $\begin{array}{llll}2.684689 & 2.715988 & 0.688429\end{array}$ $3.2058823 .175506-0.515669$ $-0.863467-0.709935-1.926380$ $\begin{array}{llll}1.245133 & 1.314343 & -2.886327\end{array}$ $3.2157653 .144952-2.865033$ $\begin{array}{llll}1.186730 & 1.325701 & 1.827381\end{array}$ $\begin{array}{llll}3.168179 & 3.177328 & 1.841001\end{array}$ $\begin{array}{llll}4.175853 & 4.083477 & -0.513745\end{array}$

$-1.516799-0.5254812 .088470$ $0.850339-1.3675512 .027449$ $-0.341816-1.0006872 .678187$ $-0.382544-1.2013433 .740802$ $2.279874-1.6260720 .093683$ $3.494014-0.9276040 .217506$ $2.161802-2.760824-0.735998$ $4.609316-1.422255-0.464782$ $3.306115-3.215692-1.389985$ $4.522690-2.555538-1.258076$ $5.558216-0.899849-0.376258$ $3.245959-4.091656-2.026745$ $5.400308-2.923988-1.780216$ $-2.914346-0.0305300 .203379$ $-3.590551-1.172448-0.264374$ $\begin{array}{llll}-3.449651 & 1.257433 & 0.040863\end{array}$ $-4.836638-0.998712-0.867376$ $-4.6979721 .382923-0.572175$ $-5.3918820 .266285-1.018798$ $-5.374980-1.868442-1.235119$ $\begin{array}{llll}-5.126252 & 2.372663 & -0.708085\end{array}$ $-6.3613420 .381644 \quad-1.493624$ $\begin{array}{lll}-2.690116 & 2.500565 & 0.468471\end{array}$ $-3.4923543 .3637361 .449395$ $\begin{array}{lll}-2.295385 & 3.327160 & -0.764513\end{array}$ $-1.7772572 .1853940 .984076$ $-3.7717972 .8037012 .347296$ $-2.9007524 .2310371 .759675$ $-4.4131233 .7366580 .988421$ $-1.8521542 .700037-1.543491$ $-3.1764323 .816731-1.195188$ $-1.5722044 .105306-0.493231$ $-3.000788-2.569544-0.147603$ $-2.724291-3.162883-1.534118$ $-3.914001-3.5030860 .657622$ $-2.043687-2.5083780 .383720$ $-2.012517-2.545457-2.088675$ $-2.313227-4.173370-1.427996$ $-3.649825-3.241431-2.115640$ $-4.129670-3.1061701 .654632$ $-4.870189-3.6574450 .145982$ $-3.439594-4.4826050 .777679$ $\begin{array}{llll}3.650876 & 0.331617 & 1.055611\end{array}$ $\begin{array}{llll}4.046527 & 1.527743 & 0.177510\end{array}$ 
$\begin{array}{lll}4.702438 & 0.144958 & 2.159499\end{array}$ $\begin{array}{llll}2.690134 & 0.563501 & 1.529000\end{array}$ $\begin{array}{llll}3.339988 & 1.674966 & -0.643813\end{array}$ $\begin{array}{llll}4.080174 & 2.443779 & 0.777567\end{array}$ $\begin{array}{llll}5.040602 & 1.375198 & -0.257022\end{array}$ $4.511418-0.7441682 .766608$ $\begin{array}{llll}5.702533 & 0.036179 & 1.726258\end{array}$ $4.720772 \quad 1.018548 \quad 2.818953$ $0.842227-3.508692-0.862686$ $0.644950-4.4790350 .308156$ $0.702288-4.232052-2.201859$ $0.040751-2.767636-0.821720$ $0.627942-3.9547371 .269526$ $-0.306981-5.0123910 .202424$ $1.450539-5.2216050 .337137$ $0.877647-3.544756-3.034466$ $1.396470-5.075203-2.287305$ $-0.308578-4.635604-2.302348$ $-2.747617-0.4184332 .951841$ $-3.512916-1.1256462 .617552$ $2510658-0.6200743 .996546$ $-3.1837470 .5814802 .864655$ $1.892635-2.0521522 .877393$ $1.413255-2.6378983 .663673$ $2.544741-2.6994342 .288658$ $2.515180-1.2901853 .359303$ $-1.621175-0.2025050 .800337$ $1.089213-1.1491910 .740615$ $-0.099966-0.097066-0.373400$ $0.6107031 .732588-0.573680$ $0.8872142 .381173-1.763955$ $\begin{array}{llll}0.871203 & 2.437369 & 0.588419\end{array}$ $\begin{array}{llll}1.419950 & 3.666540 & -1.791968\end{array}$ $13964593.720998 \quad 0.590577$ $1.680217 \quad 4.348699-0.613316$ $2.0973535 .348294-0.633484$ $-0.469203-0.903422-1.916299$ $0.6315791 .788915-2.935191$ $1.672498 \quad 4.249067 \quad-2.966352$ 0.6046741 .8540481 .780259 $\begin{array}{llll}1.644157 & 4.340523 & 1.748737\end{array}$

$-1.381195-0.4103562 .083728$ $1.066463-0.9753352 .030838$ $-0.148511-0.6915502 .680511$ $-0.146915-0.7993343 .757299$ $2.482551-1.2439620 .090570$ $3.607927-0.4012430 .116410$ $2.486093-2.458558-0.627139$ $4.763306-0.826497-0.545812$ $3.665894-2.838322-1.265978$ $4.798564-2.032656-1.227556$ $5.645289-0.191624-0.531122$ $3.699253-3.771690-1.817453$ $5.705494-2.345533-1.735793$ $-2.856109-0.2495010 .200060$ $-3.397087-1.498530-0.156921$ $-3.5439930 .947967-0.054779$ $-4.664456-1.524898-0.739631$ $-4.8076970 .872786-0.644173$ $-5.369854-0.351719-0.979552$ $5.100273-2.480184-1.020746$ $-5.3533711 .790028-0.850113$ $-6.353729-0.392178-1.436771$ $-2.93159023037710 .248606$ $-3.8141083 .1460451 .177352$ $-2.6600343 .064295-1.057972$ $\begin{array}{lll}-1.978329 & 2.143086 & 0.762317\end{array}$ $-4.0082412 .6354042 .125794$ -3.325495 4.100406 1.398586 $-4.7811883 .3668650 .713122$ $-2.1578532 .430288-1.794233$ $-3.6009383 .408736-1.502612$ $-2.0291773 .941205-0.871235$ $-2.642476-2.8012300 .059644$ $-2.331348-3.482782-1.278043$ $-3.418452-3.7568180 .975398$ $-1.686553-25804860.548867$ $-1.709107-2.840723-1.907101$ $-1.802499-4.425676-1.097295$ $-3.254297-3.719871-1.819320$
$-3.653008-3.2989871 .941523$ $-4.363088-4.0645870 .513885$ $-2.829722-4.6609151 .162529$ $\begin{array}{llll}3.627974 & 0.942488 & 0.827985\end{array}$ $3.8648542 .086143-0.169186$ $\begin{array}{llll}4.711582 & 0.987224 & 1.915668\end{array}$ $2.654244 \quad 1.1052601 .303486$ $3.1308142 .072932-0.979325$ $\begin{array}{llll}3.801995 & 3.053191 & 0.341725\end{array}$ $\begin{array}{llll}4.862309 & 2.007281 & -0.615741\end{array}$ $\begin{array}{llll}4.638761 & 0.143456 & 2.607348\end{array}$ $\begin{array}{llll}5.710701 & 0.957340 & 1.467634\end{array}$ $\begin{array}{llll}4.634604 & 1.915523 & 2.490724\end{array}$ $1.259917-3.360186-0.651632$ $1.181548-4.2212620 .614889$ $1.194516-4.230447-1.906500$ $0.380064-2.712986-0.671902$ $1.105167-3.6070381 .518255$ $0.298295-4.8696850 .577774$ $2.068546-4.8584840 .707158$ $1.285750-3.619126-2.808944$ $1.979432-4.994685-1.916905$ $0.235316-4.753092-1.946551$ $-2.601619-0.3698852 .967496$ $-3.288016-1.1826442 .711174$ $-2.326004-0.4563324 .018604$ $-3.1489960 .5648892 .811572$ $2.196980-1.4553112 .908150$ $1.803613-2.0152133 .758397$ $2.907954-2.0770482 .361553$ $2.736804-0.5860813 .299868$ $-1.543152-0.2172830 .776350$ $1.255315-0.8485570 .723524$ $-0.065000-0.036039-0.445796$ $0.415944 \quad 1.837393-0.816088$ $0.595345 \quad 2.427333-2.059414$ $\begin{array}{llll}0.612420 & 2.669327 & 0.269669\end{array}$ $0.969438 \quad 3.751841 \quad-2.238171$ $\begin{array}{llll}0.980210 & 4.003181 & 0.149077\end{array}$ $1.1642194 .548074-1.110817$ $1.4594615 .588224-1.193055$ $-0.371591-1.029740-1.891158$ $0.384251 \quad 1.682415-3.156069$ 0.4416912 .1688751 .515033 $\begin{array}{llll}1.170551 & 4.741732 & 1.249579\end{array}$ $\begin{array}{llll}1.095373 & 4.147720 & -3.238999\end{array}$

$\begin{array}{llll}1.823719 & 0.406242 & -0.268499\end{array}$ $-22284551.4149070 .648955$ $3.087400-0.1668351 .061016$ $\begin{array}{llll}0.958004 & 1.745517 & 0.829221\end{array}$

$-1.1746531 .481822-1.107838$ $-3.355997-0.070719-0.102628$ $4.378855-0.5655510 .590203$ $4.681731-1.9191570 .379496$ $5.970724-2.248105-0.045535$ $6.225818-3.291006-0.214949$ $6.923529-1.262470-0.268454$ $7.923283-1.536416-0.591249$ $\begin{array}{llll}6.589025 & 0.076721 & -0.100496\end{array}$ $\begin{array}{llll}7.330819 & 0.844410 & -0.303434\end{array}$ $\begin{array}{llll}5.311232 & 0.452222 & 0.313745\end{array}$ $3.622628-2.9991580 .507109$ $2.717326-2.5449970 .926825$ $4.045933-4.1483871 .428339$ $4.295213-3.7974882 .434428$ $4.924105-4.6686851 .031643$ $3.238101-4.8822251 .513414$ $3.280135-3.541286-0.889317$ $3.142854-2.730268-1.609354$ $2.364275-4.143019-0.857442$ $4.091358-4.177270-1.261813$ $\begin{array}{llll}4.953044 & 1.921831 & 0.471344\end{array}$ $\begin{array}{llll}3.868347 & 1.995097 & 0.582622\end{array}$ $\begin{array}{llll}5.592861 & 2.510687 & 1.733720\end{array}$ $\begin{array}{llll}5.258105 & 1.986322 & 2.635107\end{array}$ $\begin{array}{llll}5.328247 & 3.568193 & 1.840059\end{array}$ $\begin{array}{llll}6.685266 & 2.437870 & 1.685486\end{array}$ $\begin{array}{llll}5.327274 & 2.737144 & -0.768842\end{array}$ $4.8515352 .316439-1.657233$ $\begin{array}{llll}6.411470 & 2.770468 & -0.922220\end{array}$ 

$\mathrm{Ga}$ $\begin{array}{lll}-1.783435 & 0.380108 & 0.261682\end{array}$ $2.2659591 .218097 \quad-0.712077$ $-3.055222-0.274271-1.024291$ $-0.8961621 .614039-0.937760$ $\begin{array}{llll}1.212188 & 1.457066 & 1.031106\end{array}$ $3.383437-0.1938340 .182397$ $-4.360127-0.596043-0.532031$ $-4.702603-1.917190-0.207281$ $-6.003319-2.1724430 .232661$ $-6.288490-3.1893810 .489650$ $-6.929884-1.1457530 .359670$ $-7.939128-1.3630220 .695832$ $\begin{array}{lll}-6.556973 & 0.163974 & 0.078058\end{array}$ $\begin{array}{llll}-7.278771 & 0.966246 & 0.205408\end{array}$ $\begin{array}{lll}-5.266432 & 0.466830 & -0.355311\end{array}$ $-3.674871-3.033932-0.232347$ $-2.751776-2.641310-0.674589$ $-4.122428-4.240893-1.064079$ $-4.349676-3.965773-2.098563$ $-5.020530-4.701222-0.638688$ $-3.336239-5.002531-1.078518$ $-3.364525-3.4722331 .207235$ $-3.208131-2.6109121 .861851$ $-2.467958-4.1023251 .235359$ $-4.199978-4.0503091 .618773$ $-4.8670541 .906553-0.639686$ $-3.7799841 .940142-0.746183$ $-5.4806892 .397178-1.955941$ $-5.1524691 .786439-2.803761$ $-5.1869533 .433839-2.152826$ $-6.5750462 .357388-1.913067$ $\begin{array}{lll}-5.2278382 .839241 & 0.519026\end{array}$ $\begin{array}{lll}-4.768855 & 2.487726 & 1.445521\end{array}$ $\begin{array}{lll}-6.311710 & 2.9141780 .659321\end{array}$ $\begin{array}{llll}-4.8553513 .848273 & 0.308635\end{array}$ $-2.805069-0.248158-2.331789$ $-1.7278260 .448419-2.889332$ $\begin{array}{llll}-1.605630 & 0.362627 & -3.960492\end{array}$ $-0.9584391 .447258-2.257181$ $-0.2292982 .403761-3.171236$ $\begin{array}{llll}0.841797 & 2.411282 & -2.937362\end{array}$ $-0.6152793 .421781-3.054604$ $-0.3624072 .106549-4.211208$ $-3.761368-0.951612-3.261055$ $-3.832005-2.010837-2.994530$ $-3.435418-0.863680-4.297580$ $-4.768093-0.533141-3.165291$ $0.9598743 .898869 \quad 1.473176$ $\begin{array}{llll}1.734621 & 4.158685 & 0.736347\end{array}$ $-0.0644645 .0300441 .591929$ $-0.7847424 .7752122 .381068$ 0.4303685 .9576991 .897788 $-0.8152985 .2354680 .273435$ $\begin{array}{lll}-1.5986655 .990804 & 0.394316\end{array}$ $\begin{array}{lll}-0.1165765 .618450 & -0.483231\end{array}$ $-1.4224863 .922412-0.225946$ $-1.9181024 .064148-1.194614$ $\begin{array}{lll}-2.184804 & 3.553634 & 0.473726\end{array}$ $-0.3394382 .842389-0.365425$ $0.4536123 .216500-1.021855$ 0.2831102 .5938561 .024853 $-0.5550522 .3912701 .703347$ $\begin{array}{lll}1.379527 & 0.782759 & 2.165570\end{array}$ $2.259616-0.3061762 .301148$ $2.224985-0.8351573 .244848$ $3.191549-0.7679241 .367710$ $4.021760-1.9750041 .735510$ $5.087371-1.7272041 .774908$ $3.899241-2.7453310 .965177$ $3.715263-2.3823222 .700399$ $0.5715191 .133555 \quad 3.400438$ $\begin{array}{lll}-0.505309 & 1.037726 & 3.224322\end{array}$ $\begin{array}{llll}0.758725 & 2.163592 & 3.718124\end{array}$ $\begin{array}{llll}0.833332 & 0.469026 & 4.224989\end{array}$ $4.553186-0.505904-0.586727$ $4.459454-1.285035-1.751849$ $5.609910-1.465986-2.526491$ $5.547883-2.064210-3.431875$ $6.820674-0.900774-2.159725$ $7.704414-1.056488-2.770999$ 

$\begin{array}{llll}5.400662 & 2.446328 & 0.533499\end{array}$ $\begin{array}{llll}4.415123 & 2.399111 & 0.060130\end{array}$ $\begin{array}{llll}6.100596 & 2.847849 & -0.207345\end{array}$ 5.3508553 .1364591 .382214 7.2152641 .0989721 .647905 $\begin{array}{llll}7.580705 & 0.098120 & 1.899717\end{array}$ $\begin{array}{llll}7.155784 & 1.684977 & 2.570110\end{array}$ $\begin{array}{llll}7.959692 & 1.581245 & 1.005826\end{array}$ $1.478192 \quad 3.738878 \quad 2.424145$ $-0.815840-1.1378551 .068506$ $-0.920484-1.5584742 .381628$ $0.021786-1.8787330 .255137$ $-0.209705-2.6479122 .871616$ $0.740158-2.9727550 .716806$ $0.630705-3.3695752 .040965$ $1.186320-4.2227002 .411552$ $-2.6362941 .3762691 .485636$ $-1.762016-0.9437993 .229549$ $-0.348027-3.0006964 .152971$ $1.543836-3.650046-0.112372$ $0.139388-1.526353-1.046003$

$1.7883380 .361843-0.306664$ $\begin{array}{lll}-2.2586551 .101922 & 0.769150\end{array}$ $3.059142-0.4286930 .905040$ 0.9046101 .4618861 .022782 $-1.2139391 .529394-0.942155$ $-3.382168-0.204620-0.266706$ $\begin{array}{llll}4.362335 & -0.705217 & 0.382451\end{array}$ $4.698439-1.988182-0.075075$ $5.998383-2.203803-0.538050$ $6.278771-3.190785-0.896977$ $6.930233-1.174244-0.559215$ $7.938812-1.361311-0.915101$ $\begin{array}{llll}6.563742 & 0.101844 & -0.145526\end{array}$ $\begin{array}{llll}7.289776 & 0.909147 & -0.189815\end{array}$ $\begin{array}{llll}5.274199 & 0.365489 & 0.315666\end{array}$ $3.664558-3.096028-0.164671$ $2.743886-2.7465530 .317053$ $4.105876-4.3857730 .535648$ $4.336981-4.2216741 .592601$ $5.000048-4.8052530 .062306$ $3.314572-5.1396250 .472620$ $3.350080-3.379281-1.641651$ $3.201347-2.452604-2.201607$ $2.446495-3.992478-1.735596$ $4.180797-3.918971-2.111283$ $\begin{array}{llll}4.880939 & 1.769773 & 0.747406\end{array}$ $\begin{array}{llll}3.793071 & 1.799177 & 0.844418\end{array}$ $\begin{array}{llll}5.482125 & 2.112458 & 2.115117\end{array}$ $\begin{array}{llll}5.138298 & 1.418017 & 2.889101\end{array}$ $\begin{array}{llll}5.193792 & 3.124881 & 2.417864\end{array}$ $\begin{array}{llll}6.576631 & 2.067529 & 2.080978\end{array}$ $5.2592692 .818255-0.301219$ $4.8099262 .569212-1.264860$ $\begin{array}{llll}6.345001 & 2.902980 & -0.420473\end{array}$ $\begin{array}{llll}4.887368 & 3.800868 & 0.010659\end{array}$ $2.807499-0.5448662 .207136$ $\begin{array}{lll}1.731640 & 0.089544 & 2.836689\end{array}$ $1.609038-0.1111613 .892317$ $0.966873 \quad 1.154257 \quad 2.316411$ $\begin{array}{llll}0.243710 & 2.010127 & 3.330005\end{array}$ $-0.8277332 .0484943 .100989$ $\begin{array}{llll}0.635275 & 3.032708 & 3.323579\end{array}$ $\begin{array}{llll}0.377054 & 1.601430 & 4.331415\end{array}$ $3.760797-1.3475843 .055896$

$3.830307-2.3716652 .676133$ $3.432712-1.3722954 .095189$ $4.768534-0.9230683 .008533$ $-0.9474314 .002589-1.120743$ $-1.7194344 .184464-0.358115$ $0.0824695 .135054-1.118484$ $0.800163 \quad 4.963507-1.932126$ $-0.4081586 .092760-1.321234$ $\begin{array}{llll}0.836655 & 5.193129 & 0.212761\end{array}$ $\begin{array}{llll}1.622569 & 5.954454 & 0.172764\end{array}$ $0.1407135 .494070 \quad 1.008109$ $\begin{array}{llll}1.439445 & 3.831321 & 0.564284\end{array}$ $\begin{array}{llll}1.937129 & 3.864402 & 1.541662\end{array}$ $\begin{array}{llll}2.198767 & 3.537749 & -0.172922\end{array}$ $\begin{array}{llll}0.351802 & 2.747028 & 0.588198\end{array}$ $-0.4372333 .0518681 .284069$ $-0.2770812 .653046-0.818056$ $0.5571882 .518746-1.518095$ $-1.3849580 .978747-2.140934$ $-2.267924-0.087342-2.389900$ $-2.228564-0.519259-3.381258$ $-3.194900-0.647883-1.507868$ $-4.024105-1.810939-1.998871$ $-5.091445-1.567620-1.995614$ $-3.885597-2.662548-1.322614$ $-3.728424-2.104694-3.007384$ $-0.5771941 .456477-3.332659$ $\begin{array}{llll}0.498161 & 1.317518 & -3.176636\end{array}$ $-0.7438512 .520122-3.526401$ $\begin{array}{llll}-0.858521 & 0.898390 & -4.226625\end{array}$ $-4.545493-0.6039570 .470991$ $-4.442138-1.5111681 .538458$ $-5.586334-1.7815882 .296112$ $-5.516669-2.4791963 .126739$ $-6.800702-1.1811042 .005198$ $-7.679468-1.4072622 .601476$ $-6.889117-0.2807510 .947273$ $\begin{array}{llll}-7.841571 & 0.192764 & 0.730578\end{array}$ $\begin{array}{llll}-5.772179 & 0.034530 & 0.176863\end{array}$ $-3.139259-2.2112671 .877110$ $-2.417485-1.9717301 .094399$ $-3.309478-3.7350441 .892450$ $-3.743975-4.0990660 .956595$ $-3.959569-4.0538312 .714647$ $-2.337167-4.2187312 .025588$ $-2.557089-1.7352653 .214622$ $-1.586747-2.2129993 .388953$ $-3.224373-1.9989174 .043856$ $-2.411599-0.6505023 .225311$ $-5.8486281 .106345-0.902636$ $\begin{array}{llll}-5.137969 & 0.852081 & -1.696462\end{array}$ $-5.4037872 .451162-0.310602$ $\begin{array}{lll}-4.414125 & 2.352742 & 0.145731\end{array}$ $\begin{array}{lll}-6.0969342 .768264 & 0.476160\end{array}$ $-5.3615123 .230414-1.078769$ $-7.2289581 .233289-1.549230$ $-7.5967440 .266186-1.907249$ $-7.1779691 .918130-2.401194$ $-7.9673901 .640618-0.850763$ $-1.4686173 .949372-2.082094$ $0.821561-1.053410-1.278200$ $0.923581-1.340723-2.631900$ $-0.018035-1.884052-0.563248$ $0.230637-2.359024-3.268788$ $-0.738642-2.917342-1.150542$ $-0.619175-3.158357-2.508496$ $-1.187023-3.971982-2.946531$ $2.6463891 .494973-1.402584$ $1.781480-0.615487-3.379065$ $-1.549715-3.678199-0.400768$ $-0.140040-1.6895090 .769619$ $0.370542-2.527402-4.330304$

$-1.966846-0.482863-0.255324$ $1.607149-0.207531-0.321836$ $-3.370689-0.7322391 .068154$ $-0.869575-1.9419400 .395357$ $0.549983-0.845236-1.839076$ $2.2740891 .401980-1.145634$ $-4.715876-0.6056630 .593652$ $\begin{array}{llll}-5.464482 & 0.564964 & 0.786702\end{array}$ 

2.1781163 .0810593 .215690 $\begin{array}{llll}1.109252 & 1.833415 & 2.532431\end{array}$ $\begin{array}{llll}4.860085 & 0.937141 & -2.290701\end{array}$ $4.3150131 .572748-3.004021$ $4.277455-0.483307-2.420097$ $3.196016-0.488421-2.589119$ $4.501588-1.078874-1.533342$ $4.728762-0.988523-3.280369$ $\begin{array}{llll}6.322340 & 0.924273 & -2.743798\end{array}$ $\begin{array}{llll}6.776920 & 1.917382 & -2.686526\end{array}$ $6.3822910 .581710-3.780512$ $\begin{array}{llll}6.919539 & 0.236618 & -2.134399\end{array}$ $1.060379-2.704413-3.746736$ $-1.6914841 .468640-0.355379$ $-1.6923122 .198021-1.529090$ $-1.5742922 .2051210 .816691$ $-1.5132933 .577675-1.569216$ $-1.4754423 .5872170 .830470$ $-1.4079644 .273833 \quad-0.376417$ $-2.695527-1.046926-1.806538$ $-1.9351711 .602174-2.705533$ $-1.4471374 .2836461 .970220$ $-1.6045021 .5858382 .006430$ $-1.4491714 .230650-2.731838$ $-1.2633645 .597107-0.378500$ $2.982915-1.2527680 .692675$ $3.369491-0.7125711 .914709$ $3.573855-2.4699730 .390212$ $4.267358-1.3212532 .783283$ $4.469468-3.1265341 .223049$ $4.820329-2.5435112 .432523$ $\begin{array}{llll}0.484183 & 0.367697 & 0.964404\end{array}$ $\begin{array}{llll}2.861089 & 0.452300 & 2.319665\end{array}$ $4.595830-0.7533793 .940463$ $5.678054-3.1519543 .243185$ $4.997290-4.2987890 .874012$ $3.297544-3.095932-0.768863$

$7 b$

$\mathrm{Ga}$

$\mathrm{N}$

$\mathrm{N}$

$\begin{array}{lll}-1.947534 & 0.350613 & 0.302261\end{array}$ $\begin{array}{lll}-3.428878 & 0.459238 & -0.949874\end{array}$ $-0.9330401 .795119-0.514955$ $\begin{array}{llll}0.653296 & 0.906987 & 1.665159\end{array}$ $2.505718-1.2361080 .932226$ $\begin{array}{llll}-4.743017 & 0.322299 & -0.399192\end{array}$ $-5.455108-0.884961-0.468378$ $-6.727905-0.9351060 .108190$ $-7.292524-1.8627630 .062201$ $\begin{array}{llll}-7.277335 & 0.171457 & 0.737777\end{array}$ $-8.2695660 .1137601 .174776$ $-6.5507731 .3550620 .810157$ $\begin{array}{lll}-6.9834372 .217244 & 1.309002\end{array}$ $\begin{array}{llll}-5.273378 & 1.449396 & 0.262224\end{array}$ $-4.893484-2.130815-1.130188$ $-3.921268-1.879663-1.564934$ $-5.806000-2.635440-2.256046$ $-6.030544-1.848475-2.982318$ $-6.759693-2.996171-1.855705$ $-5.332078-3.469012-2.784282$ $-4.677526-3.243469-0.095089$ $-4.034391-2.9047650 .722722$ $-4.213320-4.117294-0.565636$ $-5.633679-3.5604140 .336445$ $\begin{array}{lll}-4.503086 & 2.757937 & 0.322521\end{array}$ $\begin{array}{lll}-3.4506532 .525831 & 0.156083\end{array}$ $-4.9531053 .699828 \quad-0.799609$ $-4.8102353 .240240-1.784102$ $-4.3777174 .632157-0.773431$ $-6.0146103 .951145-0.694031$ $-4.5988063 .4309861 .691871$ $-4.2555312 .7471762 .471713$ $-5.6190133 .7563061 .921566$ $-3.9684834 .3276081 .711048$ $-3.1979260 .766191-2.214665$ $-1.9624541 .284160-2.649141$ $-1.8442101 .403522-3.718375$ $-0.9940311 .904377-1.849111$ $-0.0501592 .826381-2.590969$ $\begin{array}{llll}0.932841 & 2.901272 & -2.125944\end{array}$ $-0.4727083 .835071-2.649770$ $0.085508 \quad 2.457875-3.608709$ 
$\begin{array}{llll}2.316001 & 0.347298 & -0.398061\end{array}$ $\begin{array}{llll}-2.003745 & 0.323387 & 0.003621\end{array}$ $\begin{array}{llll}3.589056 & 0.109531 & 1.019673\end{array}$ $\begin{array}{llll}1.107337 & 1.492544 & 0.588982\end{array}$ $-0.8584810 .752476-1.514137$ $-2.699646-1.357263-0.610177$ $\begin{array}{llll}4.965107 & 0.006552 & 0.646034\end{array}$ $5.576756-1.2467330 .491082$ $6.933306-1.2828220 .160892$ $\begin{array}{llll}7.425669 & -2.243891 & 0.036047\end{array}$ $7.656138-0.111127-0.023470$ $8.712104-0.157507-0.272006$ $\begin{array}{llll}7.020773 & 1.120864 & 0.088039\end{array}$ $\begin{array}{llll}7.585431 & 2.033400 & -0.083941\end{array}$ $\begin{array}{llll}5.665545 & 1.204100 & 0.406942\end{array}$ $4.777411-2.5345500 .576393$ $\begin{array}{lll}3.766642 & -2.2896570 .923019\end{array}$ $5.378582-3.5493661 .554693$ $5.468647-3.1405412 .565775$ $6.377415-3.8638531 .233633$ $4.750144-4.4444191 .603754$ $4.665047-3.154404-0.825266$ $4.406074-2.400587-1.573186$ $3.900474-3.939864-0.843360$ $5.621435-3.600273-1.122791$ $\begin{array}{llll}4.976879 & 2.556539 & 0.503035\end{array}$ $\begin{array}{llll}3.898965 & 2.3830160 .550388\end{array}$ 5.3890803 .2925891 .782836 $\begin{array}{llll}5.125773 & 2.718250 & 2.677369\end{array}$ $\begin{array}{llll}4.887052 & 4.264094 & 1.845312\end{array}$ $6.4707863 .468307 \quad 1.799105$ $\begin{array}{llll}5.234886 & 3.417486 & -0.735973\end{array}$ $\begin{array}{llll}4.918562 & 2.888402 & -1.637347\end{array}$ $\begin{array}{lll}6.291996 & 3.689740 & -0.829572\end{array}$ $\begin{array}{llll}4.662900 & 4.349632 & -0.662366\end{array}$ $\begin{array}{llll}3.230699 & 0.212327 & 2.299184\end{array}$ $\begin{array}{llll}1.995754 & 0.720036 & 2.704649\end{array}$ $\begin{array}{llll}1.795987 & 0.7020513 .767250\end{array}$ $\begin{array}{llll}1.078064 & 1.446660 & 1.916029\end{array}$ $\begin{array}{lll}0.056510 & 2.256259 & 2.683610\end{array}$ $-0.9437301 .8510692 .506698$ $0.069913 \quad 3.3102432 .392727$ 0.2612932 .1996563 .752477 $4.226766-0.1874273 .357819$ $4.521906-1.2324053 .219337$ $3.806766-0.0634194 .356142$ $\begin{array}{llll}5.138232 & 0.412855 & 3.275266\end{array}$ $-0.8383503 .139553-2.261521$ $-1.7173683 .404166-1.668290$ $0.0430554 .376105-2.428869$ $0.9301414 .122544 \quad-3.024949$ $-0.5022095 .154443-2.972344$ $0.4800684 .881160-1.054354$ $1.1509095 .741423-1.148768$ $-0.4038015 .222828-0.498021$ $\begin{array}{llll}1.177252 & 3.765168 & -0.280102\end{array}$ $\begin{array}{llll}1.469109 & 4.099362 & 0.722853\end{array}$ $2.100113 \quad 3.468322-0.797012$ $\begin{array}{llll}0.322862 & 2.489950 & -0.142849\end{array}$ $-0.6026562 .7191870 .406614$ $-0.0832872 .007269-1.549986$ $0.853268 \quad 1.829675-2.089503$ $-0.765432-0.102486-2.539190$ $-1.379640-1.368116-2.605062$ $-1.112305-1.968889-3.463429$ $-2.242123-1.979881-1.684242$ $-2.636164-3.417399-1.906839$ $-3.694093-3.587512-1.693479$ $-2.061134-4.037394-1.207617$ $-2.410678-3.737509-2.925155$ $\begin{array}{llll}0.077819 & 0.268658 & -3.741285\end{array}$ $\begin{array}{llll}1.123947 & 0.447749 & -3.478112\end{array}$ $-0.3019711 .178515-4.214278$ $0.056096-0.535416-4.477200$ $-3.795090-1.8147890 .193489$ $-3.555702-2.3303161 .476744$ 
$-5.342654-1.140481-1.730583$ $-4.728844-1.787444-2.370430$ $-4.9203110 .308025-2.009843$ $\begin{array}{llll}-3.835873 & 0.431784 & -2.080260\end{array}$ $-5.2965980 .962576-1.220440$ $-5.3399230 .647911-2.962495$ $-6.795787-1.339457-2.169296$ $-7.154480-2.349398-1.949135$ $-6.879117-1.172927-3.247051$ $-7.461804-0.623158-1.675626$ $-1.2157202 .782257-3.224566$ $1.756171-1.401013-1.115656$ $2.005787-1.867017-2.399648$ $1.103706-2.292401-0.282327$ $1.602849-3.103746-2.879158$ $0.662151-3.536699-0.721058$ $0.902293-3.946995-2.020609$ $0.558262-4.927368-2.332453$ $3.042301 \quad 1.393470-1.667978$ $2.737466-1.096679-3.232197$ $0.004355-4.3447940 .125881$ $0.926839-1.9786731 .010595$ $1.848182-3.398669-3.892911$ $-3.3126081 .6334220 .733565$ $\begin{array}{lll}-3.689821 & 1.5096712 .069842\end{array}$ -3.931660 2.6723940 .059496 $-4.5875192 .3438312 .717935$ $-4.8357223 .5395850 .663840$ $-5.1678233 .3834471 .996991$ $-4.8240562 .1703583 .760968$ $-5.8775564 .0677232 .448009$ $-0.952999-0.1703741 .365849$ $-3.7074632 .865146-1.254060$ $-5.3861324 .517969-0.066118$ $-3.1494040 .5101272 .790466$

$\begin{array}{crrr}\text { Int1a } & & & \\ \mathrm{Ga} & 1.05593700 & 0.19167900 & 0.04373000 \\ \mathrm{Ga} & -1.21945800 & 0.85209500 & -0.36543300 \\ \mathrm{~N} & 1.95197500 & -1.21216500 & 1.03290400 \\ \mathrm{~N} & 1.38407400 & 1.58903200 & 1.50298600 \\ \mathrm{~N} & -1.09505900 & 2.71296400 & 0.38968600 \\ \mathrm{~N} & -2.85582000 & 0.35877800 & 0.57864000 \\ \mathrm{C} & 2.62224200 & -2.17492600 & 0.21132100 \\ \mathrm{C} & 3.93258500 & -1.86982000 & -0.20502900 \\ \mathrm{C} & 4.53533300 & -2.72182100 & -1.12801900 \\ \mathrm{H} & 5.54048100 & -2.50299000 & -1.47982600 \\ \mathrm{C} & 3.86668000 & -3.84352500 & -1.60826600 \\ \mathrm{H} & 4.34898800 & -4.49099800 & -2.33416200 \\ \mathrm{C} & 2.59042800 & -4.14434000 & -1.15182200 \\ \mathrm{H} & 2.07834700 & -5.02586600 & -1.52900900 \\ \mathrm{C} & 1.93714500 & -3.31138600 & -0.24106700 \\ \mathrm{C} & 4.70832300 & -0.69666400 & 0.38791300 \\ \mathrm{H} & 4.08874500 & -0.21345800 & 1.15198700 \\ \mathrm{C} & 5.07740700 & 0.38687900 & -0.63283100 \\ \mathrm{H} & 4.19737000 & 0.87424700 & -1.05449200 \\ \mathrm{H} & 5.64468400 & -0.03646200 & -1.46934200 \\ \mathrm{H} & 5.70226200 & 1.14552400 & -0.14829200 \\ \mathrm{C} & 5.98089300 & -1.20490200 & 1.08239300 \\ \mathrm{H} & 5.76124300 & -1.99190200 & 1.81084800 \\ \mathrm{H} & 6.48331400 & -0.38419000 & 1.60448300 \\ \mathrm{H} & 6.68670900 & -1.61709100 & 0.35312400 \\ \mathrm{C} & 0.53624700 & -3.65226400 & 0.24820200 \\ \mathrm{H} & 0.29719500 & -2.96385000 & 1.06573800\end{array}$

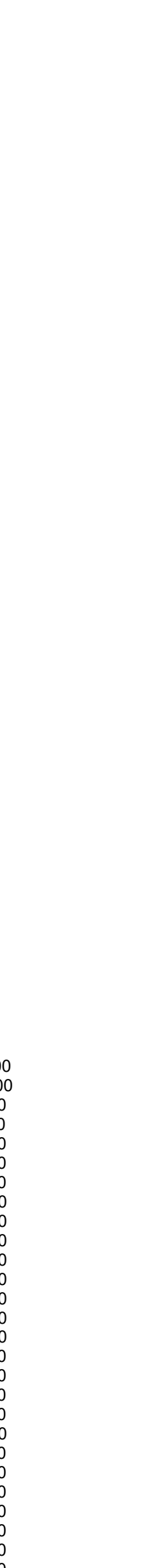

$\begin{array}{lll}0.48147100 & -5.07933700 & 0.80874800\end{array}$ $\begin{array}{lll}-0.49324500 & -5.25831000 & 1.27673900\end{array}$ $\begin{array}{lll}1.26416100 & -5.25740200 & 1.55405800\end{array}$ $\begin{array}{llll}0.60370000 & -5.82273600 & 0.01364500\end{array}$ $\begin{array}{lll}-0.54285500 & -3.46892800 & -0.83089100\end{array}$ $\begin{array}{llll}-0.21837900 & -3.87387900 & -1.79368700\end{array}$ $\begin{array}{llll}-0.79599200 & -2.41436500 & -0.97391200\end{array}$ $\begin{array}{llll}-1.46429000 & -3.97606100 & -0.52985500\end{array}$ $\begin{array}{lll}2.08537700 & -1.22589600 & 2.35426100\end{array}$ $\begin{array}{lll}1.75685700 & -0.13930300 & 3.16670400\end{array}$ $\begin{array}{lll}1.83578800 & -0.29993800 & 4.23382300\end{array}$ $\begin{array}{lll}1.50609400 & 1.19278600 & 2.75669300\end{array}$ $\begin{array}{lll}1.40920100 & 2.19645500 & 3.88987500\end{array}$ $\begin{array}{lll}0.42998200 & 2.68795500 & 3.88388700\end{array}$ $\begin{array}{lll}2.17577000 & 2.97282600 & 3.80761700\end{array}$ $\begin{array}{lll}1.53186700 & 1.69781300 & 4.85094700\end{array}$ $\begin{array}{lll}2.65884200 & -2.45936000 & 3.00236900\end{array}$ $\begin{array}{lll}2.10053400 & -3.34841900 & 2.69070400\end{array}$ $2.63257800-2.37880500 \quad 4.08982000$ $\begin{array}{lll}3.69589900 & -2.60493100 & 2.68252700\end{array}$ $\begin{array}{lll}1.29166200 & 3.01718600 & 1.18827800\end{array}$ $\begin{array}{lll}1.03066200 & 3.59052200 & 2.08337900\end{array}$ $\begin{array}{llll}2.66708600 & 3.48471800 & 0.68060300\end{array}$ $\begin{array}{llll}3.40805300 & 3.30095600 & 1.46807100\end{array}$ $\begin{array}{llll}2.94420100 & 2.84547900 & -0.16818000\end{array}$ $\begin{array}{llll}2.67835200 & 4.94693300 & 0.23427900\end{array}$ $\begin{array}{llll}2.49664100 & 5.59771600 & 1.10168800\end{array}$ $\begin{array}{llll}3.66945800 & 5.20508400 & -0.15254700\end{array}$ $\begin{array}{lll}1.60116100 & 5.20466800 & -0.82140600\end{array}$ $\begin{array}{llll}1.81365200 & 4.62513900 & -1.72520000\end{array}$ $\begin{array}{llll}1.59127700 & 6.26205600 & -1.10551400\end{array}$ $0.23094500 \quad 4.81116300-0.26771600$ $\begin{array}{llll}0.02972300 & 5.45622900 & 0.59040400\end{array}$ $\begin{array}{llll}-0.55927000 & 4.97554300 & -1.00785100\end{array}$ $\begin{array}{lll}0.22101900 & 3.32347100 & 0.11956400\end{array}$ $\begin{array}{llll}0.57789800 & 2.82971200 & -0.78265300\end{array}$ $\begin{array}{lll}-2.03608000 & 3.23392900 & 1.15210300\end{array}$ $\begin{array}{lll}-3.26193500 & 2.58486200 & 1.43205900\end{array}$ $\begin{array}{lll}-3.97665200 & 3.16875400 & 1.99564000\end{array}$ $\begin{array}{lll}-3.61709000 & 1.25335200 & 1.21363200\end{array}$ $\begin{array}{lll}-4.92530400 & 0.80381500 & 1.81949400\end{array}$ $\begin{array}{lll}-5.66095500 & 0.62044800 & 1.03129200\end{array}$ $\begin{array}{lll}-5.32129100 & 1.56680500 & 2.48978700\end{array}$ $\begin{array}{lll}-4.80392200 & -0.13160200 & 2.37193600\end{array}$ $\begin{array}{lll}-1.88213100 & 4.57816900 & 1.83683800\end{array}$ $\begin{array}{lll}-2.01879500 & 5.39690900 & 1.12571900\end{array}$ $\begin{array}{lll}-0.89591300 & 4.68721300 & 2.29255700\end{array}$ $\begin{array}{lll}-2.63119000 & 4.68193400 & 2.62197500\end{array}$ $\begin{array}{lll}-3.19595300 & -1.03545200 & 0.64975500\end{array}$ $\begin{array}{llll}-2.54093300 & -1.84918500 & 1.60998100\end{array}$ $\begin{array}{lll}-2.92796400 & -3.18961700 & 1.68258400\end{array}$ $\begin{array}{lll}-2.47749500 & -3.84266000 & 2.42007200\end{array}$ $\begin{array}{lll}-3.88182200 & -3.72596900 & 0.82263900\end{array}$ $\begin{array}{lll}-4.15060800 & -4.77523400 & 0.90051800\end{array}$ $\begin{array}{lll}-4.48042800 & -2.91997100 & -0.12946900\end{array}$ $\begin{array}{lll}-5.22197200 & -3.33994700 & -0.80351500\end{array}$ $-4.16534700 \quad-1.56086200 \quad-0.22374300$ $\begin{array}{lll}-1.42669200 & -1.32550200 & 2.52776800\end{array}$ $\begin{array}{lll}-0.58307200 & -1.07280100 & 1.87388000\end{array}$ $\begin{array}{lll}-1.76194500 & -0.05175600 & 3.33092500\end{array}$ $\begin{array}{lll}-1.65161800 & 0.86513000 & 2.74698600\end{array}$ $\begin{array}{llll}-1.07777900 & 0.02330000 & 4.18299700\end{array}$ $\begin{array}{lll}-2.78307300 & -0.08627800 & 3.72835200\end{array}$ $\begin{array}{lll}-0.92452100 & -2.39024800 & 3.50967800\end{array}$ $\begin{array}{llll}-1.71486900 & -2.68191000 & 4.21152700\end{array}$ $\begin{array}{llll}-0.09513400 & -1.97766200 & 4.09005700\end{array}$ $\begin{array}{lll}-0.56226800 & -3.29434600 & 3.01003200\end{array}$ $\begin{array}{lll}-4.92947400 & -0.70947700 & -1.22874200\end{array}$ $\begin{array}{llll}-4.66875400 & 0.34088300 & -1.06231300\end{array}$ $\begin{array}{lll}-4.54068700 & -1.05442500 & -2.66929100\end{array}$ $\begin{array}{lll}-4.74112000 & -2.11136700 & -2.87970100\end{array}$ $\begin{array}{lll}-3.48726700 & -0.85955300 & -2.85267500\end{array}$ $\begin{array}{lll}-5.12725100 & -0.45128500 & -3.37026100\end{array}$ $-6.44910800-0.87010400-1.06185500$ $\begin{array}{llll}-6.77656800 & -1.85374900 & -1.41505600\end{array}$ $\begin{array}{llll}-6.97317000 & -0.11873100 & -1.66082800\end{array}$ $\begin{array}{lll}-6.77429900 & -0.77312300 & -0.02154400\end{array}$ $\begin{array}{llll}1.67079200 & 0.17491900 & -2.01331700\end{array}$ $\begin{array}{llll}0.71735100 & -0.83393300 & -2.56902600\end{array}$ $\begin{array}{lll}-0.56677800 & -0.54677000 & -2.77229900\end{array}$ $\begin{array}{lll}-1.13609300 & 0.80864900 & -2.47197500\end{array}$ $\begin{array}{llll}-0.04861000 & 1.80314000 & -2.72117000\end{array}$ 
$\begin{array}{lll}1.23452600 & 1.50613800 & -2.51326100\end{array}$ $\begin{array}{lll}2.93476400 & -0.12245000 & -2.50151100\end{array}$ $\begin{array}{llll}2.17534900 & 2.46797500 & -2.43159600\end{array}$ $\begin{array}{llll}-0.44966100 & 3.07922700 & -2.85825400\end{array}$ $\begin{array}{llll}-2.21215700 & 1.06975500 & -3.30862100\end{array}$ $\begin{array}{llll}-1.45056500 & -1.49793900 & -3.10633200\end{array}$ $\begin{array}{llll}1.21120200 & -2.06506300 & -2.72567600\end{array}$

Int1a"

$\mathrm{N}$

$\mathrm{N}$

$\mathrm{N}$ $\begin{array}{llll}2.424985 & 0.176852 & -1.374398\end{array}$ $0.7343452 .457377-1.295151$ $-1.6648411 .028299-1.915761$ $-2.054238-1.494732-0.751424$ $2.814688-1.185313-1.239734$ $2.133674-2.177000-1.988428$ $2.597597-3.492388-1.907377$ $2.111999-4.273167-2.481752$ $3.673382-3.838806-1.095066$ $4.013648-4.869400-1.056136$ $4.289070-2.866748-0.322550$ $5.107897-3.1425450 .336860$ $3.873580-1.533689-0.376032$ $0.889973-1.849796-2.822105$ $0.210048-1.305731-2.149938$ $0.150735-3.104425-3.293479$ $-0.777496-2.815689-3.791656$ $-0.096720-3.781203-2.467785$ $0.755353-3.660712-4.018723$ $1.105758-0.920310-4.024611$ $0.172364-0.862026-4.596952$ $1.882395-1.314205-4.689988$ $\begin{array}{llll}1.370531 & 0.092942 & -3.719470\end{array}$ $4.554159-0.5136250 .523397$ $\begin{array}{llll}4.231086 & 0.487373 & 0.221382\end{array}$ $\begin{array}{lll}4.114626 & -0.730317 & 1.978540\end{array}$ $\begin{array}{llll}4.497537 & 0.069622 & 2.620991\end{array}$ $4.491323-1.6896602 .354773$ $3.023453-0.7350202 .063813$ $6.083787-0.5569000 .424943$ $6.431029-0.435174-0.606073$ $6.486541-1.5023580 .803695$ $\begin{array}{llll}6.517685 & 0.247678 & 1.026989\end{array}$ $\begin{array}{llll}3.066282 & 0.911752 & -2.279723\end{array}$ $\begin{array}{llll}2.669328 & 2.198280 & -2.666740\end{array}$ $3.2546992 .665823-3.447754$ $\begin{array}{llll}1.450836 & 2.834587 & -2.328132\end{array}$ $\begin{array}{llll}1.067621 & 3.995739 & -3.229073\end{array}$ $\begin{array}{llll}1.293806 & 4.948644 & -2.740614\end{array}$ $0.0048903 .993921-3.479113$

$1.634927 \quad 3.949716-4.159504$ $\begin{array}{llll}4.247055 & 0.322382 & -3.020917\end{array}$ $\begin{array}{llll}4.676616 & 1.048764 & -3.711632\end{array}$ $3.942978-0.565797-3.584112$ $5.019836-0.002041-2.317345$ $-0.5516563 .010430-0.907306$ $-0.7302982 .622903 \quad 0.109253$ $-0.6368174 .530396-0.710682$ $0.2246264 .839645-0.108265$ $-0.5899305 .066900-1.663124$ $-1.9564324 .888036-0.023022$ $\begin{array}{lll}-1.985601 & 4.422528 & 0.969807\end{array}$ $\begin{array}{lll}-2.022221 & 5.970827 & 0.125778\end{array}$ $\begin{array}{lll}-3.1399474 .390681 & -0.857135\end{array}$ $-3.1510504 .918489-1.821404$ $-4.0874884 .622345-0.359253$ $\begin{array}{llll}-3.0414902 .883960 & -1.103847\end{array}$ $-3.0979952 .349636-0.145464$ $-3.8815352 .526314-1.712564$ $-1.7144712 .486719-1.771188$ $-1.6483212 .970198-2.753108$ $-2.2145210 .448132-2.970804$ $-2.519285-0.926969-3.031360$ $-2.913104-1.297879-3.969215$ $-2.571989-1.798845-1.937769$ $-3.290372-3.114100-2.107061$ $-4.266476-3.082170-1.611437$ $-2.718908-3.923281-1.641908$ $-3.445195-3.337521-3.164044$ $-2.5885431 .270650-4.185562$ $-3.3657572 .004157-3.950111$ $\begin{array}{lll}-2.958815 & 0.627405 & -4.984067\end{array}$ $-1.7161591 .818527-4.557501$
$-2.445404-2.2218270 .419033$ $-1.535007-3.0804101 .055173$ $-1.925119-3.6976962 .245777$ $-1.228005-4.3613032 .750594$ $-3.179464-3.4732412 .794648$ $-3.466552-3.9645113 .719292$ $-4.060066-2.6007042 .165342$ $-5.029278-2.4007232 .614990$ $-3.706381-1.9484800 .984883$ $-0.149647-3.3422030 .496836$ $-0.065129-2.839207-0.471025$ $0.938164-2.7766401 .415755$ $0.837442-1.6909571 .532691$ $1.928585-2.9830010 .996801$ $0.880494-3.2290722 .411837$ $0.078525-4.8380390 .250397$ $-0.689958-5.256412-0.409435$ $0.055466-5.4034151 .188401$ $1.058365-4.995122-0.211067$ $-4.629273-0.8834660 .411421$ $-4.234921-0.555592-0.556534$ $\begin{array}{llll}-4.604226 & 0.336437 & 1.341016\end{array}$ $\begin{array}{lll}-3.573796 & 0.647708 & 1.531072\end{array}$ $-5.0585120 .0950882 .308882$ $\begin{array}{lll}-5.155990 & 1.175694 & 0.903062\end{array}$ $-6.062341-1.3800730 .201619$ $-6.672184-0.594141-0.255423$ $-6.533313-1.6500111 .152630$ $-6.097727-2.260120-0.448284$ $-0.767022-0.018106-0.444957$ $\begin{array}{llll}1.502888 & 1.224284 & 0.227724\end{array}$ $-0.7727691 .6676452 .756708$ 0.2426562 .5854722 .958621 $-0.6197640 .3470903 .149388$ $0.551913-0.0418463 .782323$ $1.4029412 .204248 \quad 3.614293$ $\begin{array}{llll}1.556443 & 0.889632 & 4.021803\end{array}$

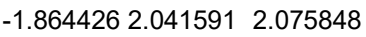
$0.714953-1.3021294 .173035$ $-1.576389-0.5404402 .885633$ $\begin{array}{llll}0.127841 & 3.811915 & 2.452432\end{array}$ 2.4059643 .0627853 .737544 $2.683430 \quad 0.501806 \quad 4.609536$

\section{Int1a'}

Ga

$\mathrm{Ga}$

$\mathrm{N}$

$\mathrm{N}$

$\begin{array}{lll}-1.233849 & 0.481399 & 0.122103\end{array}$ $1.066999-0.1145720 .267074$ $-3.056039-0.0149530 .560806$ $-1.1883782 .116101 \quad 1.274948$ $\begin{array}{lll}1.405126 & 0.908203 & 1.965486\end{array}$ $2.188502-1.6295240 .730887$ $-3.428196-1.3005260 .052886$ $-3.243357-2.4408020 .859240$ $-3.540661-3.6871700 .302335$ $-3.409131-4.5842700 .901798$ $-3.994958-3.800734-1.006717$ $-4.221986-4.779547-1.418297$ $-4.138234-2.664128-1.794938$ $-4.464922-2.763581-2.825360$ $-3.836761-1.398613-1.290080$ $-2.714178-2.3518242 .286787$ $-2.782699-1.3122932 .623314$ $-1.227687-2.7284842 .356821$ $-0.865897-2.6790883 .390055$ $-0.629910-2.0341651 .761574$ $-1.063118-3.7455251 .978729$ $-3.530203-3.2119873 .259009$ $-3.217607-3.0145644 .289138$ $-3.377697-4.2799623 .071609$ $-4.603489-3.0107873 .178706$ $-3.951658-0.149060-2.145665$ $-3.1635600 .534502-1.827750$ $-3.733298-0.413111-3.633975$ $-3.5922360 .541069-4.150180$ $-4.595487-0.915069-4.088246$ $-2.841360-1.022257-3.801145$ $-5.2839140 .571902-1.909742$ $-5.3750340 .916793-0.875258$ $-6.129820-0.088965-2.131804$ $-5.3540541 .450236-2.559759$ $-3.7871980 .6005491 .489372$ $-3.3546251 .6990462 .234664$ 


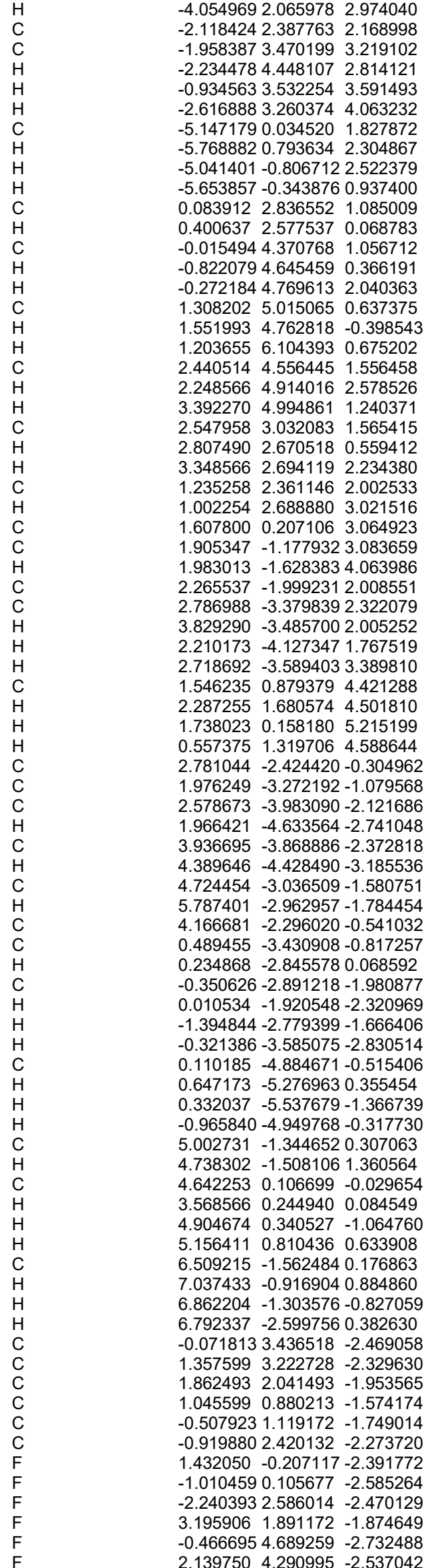

Int1b

$\mathrm{Ga}$ $\begin{array}{lll}-1.16822000 & 0.87942600 & -0.42184700\end{array}$

$\begin{array}{lll}1.97415200 & -1.24970100 & 0.96740600\end{array}$

$\begin{array}{lll}1.46998700 & 1.56905100 & 1.45666100\end{array}$

$\begin{array}{lll}-1.00765000 & 2.73960200 & 0.33850400\end{array}$

$\begin{array}{lll}-2.79161500 & 0.40976200 & 0.56655500\end{array}$

$2.54960000-2.25770200 \quad 0.13089600$

$3.86668600-2.05172600 \quad-0.32784800$

$4.38246900-2.95135500-1.25912900$

$5.39265100-2.81040000-1.63611600$

$3.62529700-4.02921400-1.70887900$

$\begin{array}{lll}4.04243100 & -4.71792200 & -2.43712400\end{array}$

$2.34255800 \quad-4.22883300-1.21949400$

$1.75621700-5.07103200-1.57763000$

$1.77190400-3.34067600 \quad-0.30420400$

$\begin{array}{lll}4.74582900 & -0.93249100 & 0.22512100\end{array}$

$\begin{array}{lll}4.19703900 & -0.41378600 & 1.01890000\end{array}$

$\begin{array}{llll}5.12105700 & 0.12782600 & -0.81968000\end{array}$

$\begin{array}{llll}4.25911900 & 0.72660600 & -1.12353500\end{array}$

$\begin{array}{llll}5.55279700 & -0.33306600 & -1.71554700\end{array}$

$\begin{array}{llll}5.86778300 & 0.81164300 & -0.40175700\end{array}$

$6.02292400-1.51697600 \quad 0.84691800$

$\begin{array}{lll}5.79681700 & -2.30382000 & 1.57324000\end{array}$

$\begin{array}{lll}6.59215800 & -0.73233100 & 1.35547800\end{array}$

$\begin{array}{llll}6.67081000 & -1.95264600 & 0.07866000\end{array}$

$0.35274300 \quad-3.56929200 \quad 0.19721100$

$0.16871500-2.85589800 \quad 1.00727600$

$\begin{array}{llll}0.19646000 & -4.98106900 & 0.77755800\end{array}$

$\begin{array}{lll}-0.79017200 & -5.08286300 & 1.24284500\end{array}$

$\begin{array}{lll}0.96182000 & -5.20115300 & 1.52991200\end{array}$

$\begin{array}{llll}0.26984900 & -5.74357900 & -0.00539900\end{array}$

$\begin{array}{lll}-0.71201300 & -3.32320800 & -0.88589600\end{array}$

$-0.40679800-3.74586400-1.84812000$

$-0.90028000 \quad-2.25639200-1.03351300$

$\begin{array}{lll}-1.66306800 & -3.77606000 & -0.58806900\end{array}$

$2.15470100-1.26289100 \quad 2.28322900$

$\begin{array}{lll}1.90104200 & -0.16204700 & 3.10312700\end{array}$

$2.02555700-0.32188500 \quad 4.16603000$

$\begin{array}{lll}1.65171700 & 1.17417500 & 2.70252400\end{array}$

$\begin{array}{lll}1.62822200 & 2.18119800 & 3.83714900\end{array}$

$\begin{array}{lll}0.65231200 & 2.67636400 & 3.89047800\end{array}$

$\begin{array}{lll}2.39002900 & 2.95544900 & 3.70570500\end{array}$

$\begin{array}{lll}1.80849500 & 1.68502900 & 4.79042500\end{array}$

$270119400-2.51635200 \quad 2.91697200$

$\begin{array}{lll}2.10495000 & -3.38474300 & 2.61749800\end{array}$

$\begin{array}{llll}2.70339700 & -2.43709100 & 4.00482600\end{array}$

$3.72509600-2.69793700 \quad 2.57333900$

$\begin{array}{lll}1.38512600 & 3.00042700 & 1.14802500\end{array}$

$\begin{array}{lll}1.12515800 & 3.57026800 & 2.04611800\end{array}$

$\begin{array}{lll}2.76800700 & 3.45490900 & 0.64569400\end{array}$

$3.50300000 \quad 3.26743100 \quad 1.43801000$

$3.04179100 \quad 2.80790100-0.19814100$

$\begin{array}{lll}2.80117700 & 4.91476300 & 0.19315000\end{array}$

$2.62566700 \quad 5.57134200 \quad 1.05749000$

$\begin{array}{lll}3.79753700 & 5.15740800 & -0.19065100\end{array}$

$\begin{array}{llll}1.73115300 & 5.18363000 & -0.86659600\end{array}$

$\begin{array}{llll}1.93601600 & 4.59754100 & -1.76768900\end{array}$

$\begin{array}{llll}1.73662400 & 6.24021900 & -1.15401500\end{array}$

$\begin{array}{llll}0.35582500 & 4.81111700 & -0.31257300\end{array}$

$\begin{array}{lll}0.16386500 & 5.46189200 & 0.54334800\end{array}$

$\begin{array}{llll}-0.43107500 & 4.98429900 & -1.05427400\end{array}$

$\begin{array}{lll}0.32203300 & 3.32393400 & 0.07646400\end{array}$

$\begin{array}{lll}0.67102800 & 2.82484100 & -0.82525600\end{array}$

$\begin{array}{lll}-1.94456700 & 3.28412100 & 1.08679300\end{array}$

$\begin{array}{lll}-3.18907200 & 2.66228200 & 1.35357600\end{array}$

$\begin{array}{lll}-3.90216100 & 3.26781000 & 1.89596200\end{array}$

$\begin{array}{lll}-3.55184100 & 1.32890000 & 1.17021800\end{array}$

$\begin{array}{lll}-4.85665700 & 0.89821000 & 1.79831200\end{array}$

$\begin{array}{lll}-5.55615400 & 0.54632900 & 1.03556700\end{array}$

$\begin{array}{lll}-5.31564600 & 1.72602800 & 2.33866600\end{array}$

$\begin{array}{lll}-4.69472700 & 0.06591800 & 2.48969100\end{array}$

$\begin{array}{lll}-1.77066200 & 4.62519700 & 1.77362900\end{array}$

$\begin{array}{llll}-1.88537400 & 5.44761500 & 1.06292800\end{array}$

$\begin{array}{lll}-0.78670900 & 4.71380500 & 2.23847700\end{array}$

$\begin{array}{lll}-2.52432700 & 4.74186600 & 2.55253200\end{array}$

$\begin{array}{lll}-3.14800800 & -0.97481800 & 0.70585200\end{array}$

$\begin{array}{llll}-2 & 50287400 & -1.75440700 & 1.69807100\end{array}$

$\begin{array}{lll}-2.92885800 & -3.07740300 & 1.84839900\end{array}$

$\begin{array}{lll}-2.48759900 & -3.70235700 & 2.61515900\end{array}$

$\begin{array}{lll}-3.91469500 & -3.62870300 & 1.03576500\end{array}$

$\begin{array}{lll}-4.21646600 & -4.66223800 & 1.17753800\end{array}$

$\begin{array}{lll}-4.50109800 & -2.85832100 & 0.04584300\end{array}$

$\begin{array}{lll}-5.26142300 & -3.29252600 & -0.59727000\end{array}$ 


$\begin{array}{rrr}-4.14114400 & -1.51935700 & -0.12836100 \\ -1.36168900 & -1.21455100 & 2.57059400 \\ -0.53816000 & -0.97660200 & 1.88629000 \\ -1.66826600 & 0.08094700 & 3.34773300 \\ -1.59560500 & 0.97562700 & 2.72514400 \\ -0.94030200 & 0.18931000 & 4.15950600 \\ -2.66638400 & 0.05184300 & 3.80010700 \\ -0.83692900 & -2.26014800 & 3.56046200 \\ -1.60666800 & -2.53026700 & 4.29311800 \\ 0.01317700 & -1.84268100 & 4.10560900 \\ -0.49937200 & -3.17809900 & 3.06729300 \\ -4.83233800 & -0.70636700 & -1.21273000 \\ -4.58441800 & 0.34942700 & -1.06434400 \\ -4.29930500 & -1.10961000 & -2.58937800 \\ -4.53664000 & -2.15908400 & -2.80020400 \\ -3.21916700 & -0.99072100 & -2.63645300 \\ -4.74573200 & -0.48936000 & -3.37310000 \\ -6.35928400 & -0.85307900 & -1.17712800 \\ -6.66694300 & -1.85769300 & -1.48575600 \\ -6.81653200 & -0.14539500 & -1.87553600 \\ -6.77743200 & -0.67239500 & -0.18157500 \\ 1.77257000 & 0.15938600 & -2.00624000 \\ 0.83418800 & -0.82679700 & -2.60009400 \\ -0.43800300 & -0.52024400 & -2.85538500 \\ -1.01948000 & 0.83128900 & -2.51581000 \\ 0.09529500 & 1.81182200 & -2.75340900 \\ 1.36889400 & 1.49138100 & -2.51053600 \\ 2.31969200 & 2.45157600 & -2.45022900 \\ -0.27822300 & 3.09338900 & -2.93382100 \\ -2.07033000 & 1.10975000 & -3.38131700 \\ -1.28273900 & -1.43756800 & -3.35001800 \\ 1.30782200 & -2.05950700 & -2.83666300 \\ 2.79611000 & -0.10789700 & -2.27089200\end{array}$

Int1b"

$\mathrm{N}$

$\mathrm{N}$

N

C

C

C

C

C

$\mathrm{H}$

C

$\mathrm{H}$

C

$\mathrm{H}$

$\mathrm{H}$

$\mathrm{H}$

H

C

$\mathrm{H}$

C

$\mathrm{H}$ $\begin{array}{llll}2.600793 & 0.270201 & -1.022654\end{array}$

$0.8650692519243-1.049001$

$-1.4277561 .077873-1.970645$

$-1.887131-1.492581-0.940775$

$3.000952-1.088771-0.892346$

$2.420380-2.066037-1.738720$

$2.895032-3.376854-1.648154$

$2.485554-4.145850-2.293367$

$3.884454-3.734587-0.736928$

$4.235293-4.761419-0.692605$

$4.399957-2.7786760 .123739$

$5.150513-3.0637930 .856477$

$3.970479-1.4502240 .065844$

$1.267468-1.729592-2.690569$

$0.512118-1.213026-2.080147$

$0.602354-2.978803-3.273458$

$-0.271066-2.687014-3.860878$

$0.277855-3.682155-2.498212$

$1.290022-3.506248-3.944231$

$1.596804-0.763312-3.836961$

$0.726603-0.694674-4.500421$

$2.441632-1.132281-4.429639$

$4.542234-0.4496861 .058483$

$\begin{array}{llll}4.233184 & 0.556149 & 0.757966\end{array}$

$3.965893-0.7188062 .455907$

$\begin{array}{llll}4.296862 & 0.054506 & 3.158754\end{array}$

$4.301233-1.6949702 .828058$

$2.871541-0.7116452 .437465$

$6.074768-0.4707201 .109736$

$6.519293-0.3172730 .121285$

$6.452948-1.4209931 .501700$

$\begin{array}{llll}6.436015 & 0.322981 & 1.771370\end{array}$

$3.3184601 .046451-1.830511$

$\begin{array}{llll}2.939527 & 2.339230 & -2.214477\end{array}$

$\begin{array}{llll}3.594731 & 2.843029 & -2.913162\end{array}$

$\begin{array}{llll}1.679772 & 2.943930 & -1.986024\end{array}$

$1.372355 \quad 4.130961-2.882470$

$1.5254445 .068833-2.339581$

$0.3429064 .121205-3.245873$

$2.037076 \quad 4.128410 \quad-3.747258$

$\begin{array}{llll}4.581602 & 0.502467 & -2.463144\end{array}$

$\begin{array}{llll}5.067074 & 1.259664 & -3.079770\end{array}$

$4.355012-0.369447-3.085218$

$\begin{array}{llll}5.283007 & 0.165008 & -1.694073\end{array}$

$-0.4650453 .039420-0.783120$

$\begin{array}{lll}-0.7431812 .614146 & 0.194836\end{array}$ $\begin{array}{llll}1.818864 & 0.242182 & -3.477173\end{array}$
$-0.6003404 .550035-0.546127$ $\begin{array}{llll}0.187806 & 4.852539 & 0.152368\end{array}$ $-0.4668055 .120625-1.470225$ $\begin{array}{llll}-1.990934 & 4.860477 & 0.012588\end{array}$ $\begin{array}{llll}-2.113140 & 4.357460 & 0.979656\end{array}$ $\begin{array}{lll}-2.0934385 .935734 & 0.192193\end{array}$ $\begin{array}{lll}-3.071081 & 4.375842 & -0.958182\end{array}$ $-2.9920504 .939302-1.898861$ $-4.0698434 .573357-0.554502$ $-2.9177772 .881148-1.246701$ $-3.0651592 .310604-0.319454$ $-3.6817282 .533662-1.953672$ $-1.5202192 .529180-1.782872$ $-1.3602593 .048460-2.735261$ $\begin{array}{lll}-1.848392 & 0.526638 & -3.097827\end{array}$ $-2.115681-0.850690-3.237365$ $-2.398854-1.194358-4.224270$ $-2.267397-1.762072-2.186084$ $-2.936530-3.082076-2.477510$ $-3.963094-3.081960-2.095868$ $-2.406241-3.897527-1.976070$ $-2.966173-3.271991-3.551876$ $-2.1067501 .385689-4.317459$ $-2.9192642 .097523-4.142410$ $-2.3762930 .765377-5.172473$ $-1.2107281 .960724-4.574519$ $-2.390010-2.2678540 .153798$ $-1.536872-3.1288730 .861869$ $-2.044759-3.7960071 .978831$ $-1.393171-4.4616572 .538690$ $-3.358646-3.6178272 .386995$ $-3.736961-4.1475313 .255909$ $-4.183060-2.7417971 .690185$ $-5.201462-2.5774712 .032699$ $-3.713484-2.0407310 .580424$ $-0.090120-3.3404330 .459416$ $0.091333-2.804865-0.477133$ $0.870295-2.7794061 .513682$ $0.716929-1.7038081 .662419$ $1.906986-2.9360631 .197151$ $0.721466-3.2761902 .478746$ $0.204403-4.8218600 .197205$ $-0.469656-5.235197-0.561644$ $0.084992-5.4180791 .108407$ $1.235585-4.942049-0.148764$ $-4.588942-0.971452-0.056239$ $-4.100381-0.607274-0.966392$ $\begin{array}{lll}-4.681894 & 0.218340 & 0.907602\end{array}$ $\begin{array}{llll}-3.682243 & 0.536850 & 1.214489\end{array}$ $-5.230457-0.0605541 .814648$ $\begin{array}{lll}-5.198779 & 1.063853 & 0.440042\end{array}$ $-5.983177-1.480127-0.432377$ $-6.553344-0.688580-0.929221$ $-6.548823-1.7850190 .454281$ $-5.934645-2.340271-1.107637$ $-0.671259-0.004913-0.447545$ $\begin{array}{llll}1.488880 & 1.246391 & 0.513328\end{array}$ $\begin{array}{lll}-1.0631351 .555155 & 2.789498\end{array}$ $-0.1101062 .4843673 .165654$ $-0.9475480 .2237723 .158376$ $0.133656-0.1586343 .939008$ 0.9521002 .0806943 .961161 $\begin{array}{llll}1.078870 & 0.764045 & 4.364198\end{array}$ $-2.0631521 .937747 \quad 1.984714$ $0.240693-1.4398104 .294358$ $-1.842665-0.6647932 .726702$ $\begin{array}{llll}1.927650 & 0.446682 & 4.956863\end{array}$ $-0.1848773 .7288182 .693158$ $\begin{array}{lll}1.883016 & 2.977128 & 4.275091\end{array}$

$\begin{array}{lll}-1.245758 & 0.457233 & 0.037135\end{array}$ $\begin{array}{llll}1.093178 & 0.063654 & 0.227598\end{array}$ $-3.012209-0.1384880 .570534$ $-1.2964512 .1740181 .068651$ 1.3943331 .2472291 .826018 $2.351864-1.3067310 .776915$ $-3.296132-1.4835700 .174065$ $-3.017814-2.5393361 .064808$ $-3.236030-3.8441410 .615534$ $-3.032709-4.6790501 .281033$ $-3.703414-4.093493-0.670058$ 

$5.122634-0.8300600 .259460$ $\begin{array}{lll}4.901726 & -0.960349 & 1.327317\end{array}$ $\begin{array}{llll}4.642837 & 0.572984 & -0.131418\end{array}$ $\begin{array}{llll}3.561795 & 0.627308 & -0.013404\end{array}$ $\begin{array}{llll}4.882133 & 0.786341 & -1.175976\end{array}$ $\begin{array}{llll}5.102556 & 1.339427 & 0.502109\end{array}$ $6.637736-0.9365490 .094050$ $7.132150-0.2162060 .752748$ $6.942119-0.703425-0.931877$ $7.007304-1.9367030 .341981$ $-0.2556233 .283200 \quad-2.726364$ $\begin{array}{llll}1.187086 & 3.145384 & -2.697449\end{array}$ $1.7211251 .986401-2.288449$ $0.9715450 .856673-1.721727$ $-0.5925630 .992221-1.874588$ $\begin{array}{llll}-1.066290 & 2.261137 & -2.427484\end{array}$ $1.8062283 .969968-3.031104$ $1.390548-0.318534-2.383645$ $-1.052971-0.072880-2.674458$ $-2.4062402 .389545-2.527132$ $3.050597 \quad 1.802379-2.347366$ $\begin{array}{lll}-0.737398 & 4.506167 & -3.017716\end{array}$

$\begin{array}{lrrr}\text { Int1c } & & & \\ \text { Ga } & 1.13033500 & 0.32971200 & -0.09215400 \\ \text { Ga } & -1.19312000 & 0.79589100 & -0.42661800 \\ \text { N } & 2.15211200 & -0.98499200 & 0.91338800 \\ \text { N } & 1.41207000 & 1.80002700 & 1.32031100 \\ \text { N } & -1.21304200 & 2.68201900 & 0.29283900 \\ \text { N } & -2.77055800 & 0.20773400 & 0.57809000 \\ \mathrm{C} & 2.79329900 & -1.97783500 & 0.10705400 \\ \mathrm{C} & 4.09653400 & -1.70798000 & -0.35718600 \\ \mathrm{C} & 4.66279200 & -2.59315200 & -1.27298600 \\ \mathrm{H} & 5.66312000 & -2.40121600 & -1.65368000 \\ \mathrm{C} & 3.96791000 & -3.71891200 & -1.70431800 \\ \mathrm{H} & 4.42211300 & -4.39428700 & -2.42281500 \\ \mathrm{C} & 2.70063900 & -3.98394800 & -1.20619100 \\ \mathrm{H} & 2.16377500 & -4.86583200 & -1.54592500 \\ \mathrm{C} & 2.08214100 & -3.11563900 & -0.30329300 \\ \mathrm{C} & 4.90907900 & -0.52975000 & 0.17116300 \\ \mathrm{H} & 4.33780600 & -0.04135400 & 0.96806500 \\ \mathrm{C} & 5.18690800 & 0.53974000 & -0.89332800 \\ \mathrm{H} & 4.26979000 & 1.05244100 & -1.19423300 \\ \mathrm{H} & 5.64373500 & 0.09988100 & -1.78714100 \\ \mathrm{H} & 5.87685700 & 1.29253800 & -0.49676000 \\ \mathrm{C} & 6.23413300 & -1.01664000 & 0.77534000 \\ \mathrm{H} & 6.07568100 & -1.81069000 & 1.51170800 \\ \mathrm{H} & 6.75486400 & -0.18908400 & 1.26769200 \\ \mathrm{H} & 6.89894900 & -1.41286300 & 0.00028200 \\ \mathrm{C} & 0.68655800 & -3.42945400 & 0.21719800 \\ \mathrm{H} & 0.45539100 & -2.70573900 & 1.00558100 \\ \mathrm{C} & 0.63965600 & -4.82956600 & 0.84323200 \\ \mathrm{H} & -0.33745800 & -4.99368400 & 1.31069600 \\ \mathrm{H} & 1.41765300 & -4.96408200 & 1.60295900 \\ \mathrm{H} & 0.77580200 & -5.60869800 & 0.08541100 \\ \mathrm{C} & -0.39973500 & -3.29664900 & -0.86208900 \\ \mathrm{H} & -0.08418700 & -3.75701300 & -1.80370300 \\ \mathrm{H} & -0.63523600 & -2.24970000 & -1.06627500 \\ \mathrm{H} & -1.32502700 & -3.77687500 & -0.52729200 \\ \mathrm{H} & 2.32363300 & -0.94203000 & 2.22875900 \\ \mathrm{C} & 1.37391000 & 4.72095300 & -2.04638200 \\ \mathrm{C} & 1.96167600 & 0.15428900 & 3.01589800 \\ \mathrm{H} & 2.08793100 & 0.03333700 & 4.08378300 \\ \mathrm{H} & 1.59781800 & 1.45277500 & 2.57922800 \\ \mathrm{C} & 1.45589900 & 2.47755700 & 3.68925700 \\ \mathrm{C} & 2.30797200 & 5.85159400 & -0.17153800 \\ \mathrm{H} & & & \end{array}$




\begin{tabular}{|c|c|c|c|}
\hline $\mathrm{H}$ & 1.09873700 & 6.36238900 & -1.47222300 \\
\hline C & -0.09702600 & 4.86402700 & -0.47238100 \\
\hline $\mathrm{H}$ & -0.27923200 & 5.52934200 & 0.37584600 \\
\hline $\mathrm{H}$ & -0.94691200 & 4.94673500 & -1.15833800 \\
\hline C & 0.03204400 & 3.39368500 & -0.04468900 \\
\hline $\mathrm{H}$ & 0.37968200 & 2.90668700 & -0.95166400 \\
\hline C & -2.18018100 & 3.15590100 & 1.04763300 \\
\hline C & -3.35800200 & 2.42877700 & 1.34700800 \\
\hline $\mathrm{H}$ & -4.11356200 & 2.97443800 & 1.89565200 \\
\hline C & -3.60188900 & 1.06651400 & 1.17915600 \\
\hline C & -4.85477100 & 0.52980700 & 1.83100100 \\
\hline $\mathrm{H}$ & -5.51545100 & 0.07475100 & 1.08893600 \\
\hline $\mathrm{H}$ & -5.39550200 & 1.32720900 & 2.34058200 \\
\hline $\mathrm{H}$ & -4.60429500 & -0.25126000 & 2.55533800 \\
\hline C & -2.10833300 & 4.52184800 & 1.70156000 \\
\hline $\mathrm{H}$ & -2.32593400 & 5.31477200 & 0.98119100 \\
\hline $\mathrm{H}$ & -1.11890700 & 4.71022200 & 2.12389900 \\
\hline $\mathrm{H}$ & -2.83973900 & 4.58573000 & 2.50758800 \\
\hline C & -3.00882800 & -1.19978900 & 0.74598000 \\
\hline C & -2.29566600 & -1.90212500 & 1.74927200 \\
\hline C & -2.61045100 & -3.25149800 & 1.93337500 \\
\hline $\mathrm{H}$ & -2.11221100 & -3.82019700 & 2.70923400 \\
\hline C & -3.55389400 & -3.89967800 & 1.14229300 \\
\hline $\mathrm{H}$ & -3.76941900 & -4.95083900 & 1.30943900 \\
\hline C & -4.20773200 & -3.20220800 & 0.14022200 \\
\hline $\mathrm{H}$ & -4.93397500 & -3.71169200 & -0.48691300 \\
\hline C & -3.95925200 & -1.84273800 & -0.06752100 \\
\hline C & -1.19778000 & -1.24931100 & 2.59845200 \\
\hline $\mathrm{H}$ & -0.42335300 & -0.91007800 & 1.89864500 \\
\hline C & -1.62971200 & -0.00906200 & 3.40223200 \\
\hline $\mathrm{H}$ & -1.73374200 & 0.88307500 & 2.78177100 \\
\hline $\mathrm{H}$ & -0.86601800 & 0.20709700 & 4.15792400 \\
\hline $\mathrm{H}$ & -2.57598100 & -0.18402700 & 3.92778700 \\
\hline C & -0.53706300 & -2.23750400 & 3.56506000 \\
\hline $\mathrm{H}$ & -1.24832900 & -2.57773200 & 4.32712800 \\
\hline $\mathrm{H}$ & 0.28779900 & -1.73748600 & 4.07903200 \\
\hline $\mathrm{H}$ & -0.13210800 & -3.12060500 & 3.05953400 \\
\hline C & -4.71613100 & -1.11451600 & -1.16793700 \\
\hline $\mathrm{H}$ & -4.55238400 & -0.03892000 & -1.04686400 \\
\hline C & -4.15389200 & -1.51010900 & -2.53524500 \\
\hline $\mathrm{H}$ & -4.31760900 & -2.57812100 & -2.72196700 \\
\hline $\mathrm{H}$ & -3.08448300 & -1.31766100 & -2.58474400 \\
\hline $\mathrm{H}$ & -4.64057700 & -0.93996000 & -3.33286100 \\
\hline C & -6.22654300 & -1.38052300 & -1.12447500 \\
\hline $\mathrm{H}$ & -6.45377900 & -2.41545900 & -1.40108100 \\
\hline $\mathrm{H}$ & -6.73755900 & -0.73317800 & -1.84382200 \\
\hline $\mathrm{H}$ & -6.65899200 & -1.20187900 & -0.13467300 \\
\hline C & 1.76922500 & 0.38004800 & -2.09640800 \\
\hline C & 0.92760900 & -0.70961500 & -2.65389600 \\
\hline C & -0.37641200 & -0.54259000 & -2.88324900 \\
\hline C & -1.08007700 & 0.74262500 & -2.52694000 \\
\hline C & -0.07043200 & 1.81771800 & -2.83096100 \\
\hline C & 1.24073600 & 1.68053300 & -2.62466900 \\
\hline $\mathrm{F}$ & -1.13488300 & -1.55349400 & -3.33820500 \\
\hline $\mathrm{F}$ & 1.51458700 & -1.89937300 & -2.86750000 \\
\hline $\mathrm{F}$ & -0.61734100 & 3.01811100 & -3.11579600 \\
\hline $\mathrm{F}$ & -2.19445800 & 0.91032900 & -3.34108500 \\
\hline $\mathrm{H}$ & 2.81225400 & 0.18949800 & -2.34953300 \\
\hline $\mathrm{H}$ & 1.90351800 & 2.53296700 & -2.73978100 \\
\hline
\end{tabular}
$\begin{array}{llll}1.991650 & 0.980167 & -3.212665\end{array}$ $4.522687-0.2429961 .329046$ $\begin{array}{llll}4.138564 & 0.770846 \quad 1.179955\end{array}$ $3.915381-0.7887752 .629457$ $4.163525-0.1304613 .470013$ $4.307346-1.7904552 .845555$ $2.824657-0.8495342 .561595$ $6.047933-0.1503671 .461739$ $\begin{array}{llll}6.518783 & 0.200254 & 0.537896\end{array}$ $6.490822-1.1197681 .714486$ $\begin{array}{llll}6.310557 & 0.547968 & 2.262818\end{array}$ $3.3209361 .615174-1.358923$ $2.8536892 .921873-1.552683$ $3.4962643 .581337-2.121599$ $1.5384323 .384931-1.305490$ $1.1748894 .678214-2.014498$ $1.2267085 .521934-1.319287$ $\begin{array}{llll}0.166458 & 4.653198 & -2.432026\end{array}$ $\begin{array}{llll}1.874923 & 4.867820 & -2.829251\end{array}$ $\begin{array}{llll}4.654471 & 1.279811 & -1.992577\end{array}$ $\begin{array}{llll}5.105102 & 2.163521 & -2.445787\end{array}$ $\begin{array}{llll}4.531757 & 0.510646 & -2.761958\end{array}$ $\begin{array}{llll}5.342863 & 0.870150 & -1.247265\end{array}$ $\begin{array}{lll}-0.660727 & 3.117670 & -0.233139\end{array}$ $-0.9472132 .5156590 .644155$ $\begin{array}{lll}-0.936039 & 4.552071 & 0.239490\end{array}$ $\begin{array}{lll}-0.2147074 .788590 & 1.029857\end{array}$ $-0.8025465 .280239-0.566342$ $\begin{array}{llll}-2.374925 & 4.658112 & 0.750637\end{array}$ -2.501863 3.993021 1.613405 $-2.5778475 .6775161 .095277$ $-3.3596974 .261579-0.352368$ $-3.2814054 .977432-1.183096$ $\begin{array}{lll}-4.390609 & 4.313938 & 0.013713\end{array}$ $-3.0659132 .851159-0.867245$ $-3.2086682 .125657-0.054767$ $-3.7620532 .568242-1.666998$ $-1.6189852 .701583-1.365297$ $-1.4584683 .382311-2.209940$ $-1.7116180 .924967-3.002785$ $-1.853397-0.426962-3.377175$ $-2.060520-0.624119-4.421459$ $-1.977151-1.507611-2.496304$ $-2.520580-2.807279-3.035895$ $-3.560949-2.945270-2.722705$ $-1.950056-3.653269-2.640458$ $-2.482632-2.818996-4.126616$ $-1.9863271 .951114-4.081582$ $-2.8639972 .559532-3.842769$ $-2.1620821 .461645-5.039735$ $-1.1320102 .628016-4.188953$ $-2.167114-2.402614-0.282481$ $-1.282555-3.3089350 .322942$ $-1.789080-4.1936761 .277599$ $-1.113719-4.8969861 .757509$ $-3.131369-4.1829521 .627985$ $-3.507451-4.8803832 .370217$ $-3.988574-3.2598711 .039926$ $-5.032242-3.2292221 .342106$ $-3.523431-2.3467590 .094784$ $0.196124-3.337941-0.011972$ $0.378861-2.642324-0.836544$ $1.044931-2.8850591 .181320$ $0.792046-1.8637031 .489451$ $2.107132-2.9090340 .915520$ $0.885602-3.5426372 .043127$ $0.634182-4.727983-0.487291$ $0.038954-5.063571-1.344135$ $0.522603-5.4733920 .307666$ $1.688178-4.707235-0.780649$ $-4.449596-1.247586-0.404044$ $-3.947745-0.697390-1.207284$ $-4.681576-0.2540480 .741511$ $-3.7250970 .0683831 .160888$ $-5.254152-0.7228371 .550086$ $\begin{array}{llll}-5.235382 & 0.625296 & 0.394087\end{array}$ $-5.779316-1.778517-0.945903$ $-6.385892-0.953761-1.333735$ $-6.360946-2.272640-0.160633$ $-5.630589-2.501422-1.754271$ $1.789621-2.670286-4.295284$ $\begin{array}{llll}1.867144 & 0.032171 & -3.737786\end{array}$ $-0.6113720 .053933-0.410611$ 

$0.5243361 .598102 \quad 4.505905$ $\begin{array}{llll}0.787230 & 0.241601 & 4.667685\end{array}$ $-2.2628131 .4501292 .192220$ $0.275847-2.0018244 .117626$ $-1.760333-1.1973102 .508132$ $-0.7245233 .2802913 .390519$ $1.1369982 .355962 \quad 4.978016$ $1.597792-0.1067175 .296832$

Int1c'

$\begin{array}{lll}-1.245170 & 0.572473 & -0.056359\end{array}$ $\begin{array}{llll}1.090884 & 0.148305 & 0.134746\end{array}$ $\begin{array}{lll}1.3 .025527 & 0.041571 & 0.509810\end{array}$ -1.295753 2.381215 0.801037 $\begin{array}{llll}1.377668 & 1.467888 & 1.627608\end{array}$ $2.314279-1.1891020 .838388$ $-3.314290-1.3351490 .243561$ $-3.040417-2.3015731 .231312$ $-3.257035-3.6434690 .908075$ $-3.055521-4.4102181 .651720$ $-3.717478-4.015676-0.349669$ $-3.879987-5.064574-0.578724$ $-3.949852-3.047159-1.319966$ $-4.281425-3.347562-2.308989$ $-3.732253-1.695323-1.051062$ $-2.500537-1.9300422 .608269$ $-2.630495-0.8535242 .758987$ $-0.992531-2.1981202 .707011$ $-0.620378-1.9446203 .706102$ $-0.449250-1.5851281 .984131$ $-0.768706-3.2542172 .509616$ $-3.247429-2.6533663 .735220$ $-2.934670-2.2592234 .707234$ $-3.029327-3.7264083 .733665$ $-4.332383-2.5351203 .646847$ $-3.951265-0.626537-2.107259$ $-3.2087590 .153815-1.937904$ $-3.741144-1.132234-3.532944$ $-3.677800-0.275804-4.210388$ $-4.572845-1.763581-3.867056$ $-2.810639-1.698975-3.618489$ $-5.3283950 .030982-1.960809$ $\begin{array}{llll}-5.427610 & 0.543751 & -0.999199\end{array}$ $-6.127695-0.714984-2.040966$

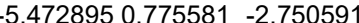
$\begin{array}{lll}-3.782521 & 0.759648 & 1.337959\end{array}$ -3.415350 1.9969771 .871758 $-4.1288012 .4398072 .554932$ -2.229761 2.742224 1.658206 $-2.1285223 .9973402 .503960$ $-2.4569454 .8750111 .939909$ $\begin{array}{lll}-1.108610 & 4.176240 & 2.848683\end{array}$

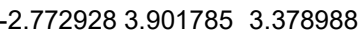
$-5.0951110 .1744071 .806918$ $\begin{array}{lll}-5.7621150 .964120 & 2.156096\end{array}$ $-4.921439-0.5183822 .638169$ $-5.585812-0.3913581 .012025$ 0.0759513 .1355150 .458782 $\begin{array}{llll}0.234297 & 2.736490 & -0.514654\end{array}$ $\begin{array}{lll}-0.267707 & 4.637901 & 0.194496\end{array}$ $-1.1101764 .753783-0.497242$ $-0.5135805 .1764381 .112502$ $1.0043645 .266530-0.377860$ $1.230275 \quad 4.821490-1.352138$ $0.840705 \quad 6.337411-0.538904$ 2.1841735 .0395890 .567298 1.9957225 .5577821 .518748 $3.1006585 .468343 \quad 0.149215$ $\begin{array}{llll}2.381466 & 3.547358 & 0.831251\end{array}$ $2.641806 \quad 3.033918-0.106109$ $3.2123003 .373198 \quad 1.525568$ $1.1183472 .890568 \quad 1.412661$ 0.8820593 .3800232 .364072 $\begin{array}{llll}1.630871 & 0.984715 & 2.828794\end{array}$ $2.019772-0.3534023 .082583$ $2.134124-0.6204694 .124522$ $2.426397-1.3238392 .158897$

$3.044370-2.5903082 .698400$ $4.089869-2.6788012 .386699$ $2.518501-3.4605862 .292364$ $2.996613-2.6140923 .787366$ $\begin{array}{llll}1.532233 & 1.877797 & 4.048930\end{array}$ 2.2228732 .7238343 .979735 $1.7703701 .317995 \quad 4.952928$ $0.518588 \quad 2.280102 \quad 4.150816$ $2.951518-2.116142-0.050537$ $2.201945-3.143506-0.640959$ $2.845616-3.995118-1.543686$ $2.275262-4.787973-2.020891$ $4.191370-3.840931-1.836003$ $4.676957-4.511414-2.538573$ $4.924657-2.824891-1.227309$ $5.978861-2.720060-1.460788$ $4.323586-1.942610-0.332384$ $0.729515-3.345118-0.331696$ $0.437071-2.6176740 .428310$ $-0.143696-3.092875-1.566713$ $0.160541-2.188059-2.092898$ $-1.191856-2.980241-1.265651$ $-0.076134-3.937239-2.264370$ $0.447238-4.7357360 .246718$ $1.007425-4.9185651 .170579$ $0.713709-5.523278-0.466934$ $-0.622412-4.8321180 .465088$ $5.097434-0.7971610 .309656$ $4.853478-0.7818671 .380651$ $\begin{array}{llll}4.632537 & 0.539508 & -0.279307\end{array}$ $\begin{array}{llll}3.553084 & 0.623552 & -0.168990\end{array}$ $\begin{array}{llll}4.866312 & 0.596116 & -1.345589\end{array}$ $\begin{array}{llll}5.102410 & 1.386364 & 0.232767\end{array}$ $6.614402-0.9326050 .189964$ $7.101683-0.1333180 .756409$ $6.939767-0.839753-0.851734$ $6.971939-1.8925990 .576126$ $-0.3188963 .057662 \quad-3.222949$ $\begin{array}{llll}1.125344 & 2.983076 & -3.057033\end{array}$ $1.6895951 .919127-2.469267$ $0.9772380 .789995-1.851035$ $-0.5820580 .887051-2.033360$ $-1.0656932 .020635-2.818307$ $1.444177-0.406327-2.452292$ $-1.038855-0.314356-2.611473$ $-2.3950882 .000162-3.058749$ $\begin{array}{llll}3.035858 & 1.835317 & -2.390564\end{array}$ $-0.7788593 .918977-3.693053$ $\begin{array}{llll}1.759593 & 3.783274 & -3.424350\end{array}$

$\begin{array}{lll}1.073286 & 0.361813 & -0.189306\end{array}$ $-1.184323-0.4740530 .349650$ $\begin{array}{llll}1.464096 & 2.069771 & 0.687944\end{array}$ $1.484633-0.5853591 .673010$ $-1.310430-1.4613132 .060520$ $-3.101880-0.5774260 .057540$ $\begin{array}{llll}0.598803 & 3.167709 & 0.379361\end{array}$ $\begin{array}{llll}0.500836 & 3.371640 & 1.241591\end{array}$ $-1.3232694 .4803921 .026732$ $-2.1571664 .6680631 .699313$ $-1.0709295 .363223-0.017064$ $-1.7099216 .228906-0.166113$ $-0.0025495 .127426-0.874479$ $0.1846055 .813958-1.696570$ $\begin{array}{llll}0.844575 & 4.030546 & -0.700721\end{array}$ $-0.7394402 .4828592 .459286$ $-0.1197671 .5823772 .374471$ $-2.1956312 .0301822 .587323$ $-2.2935841 .3135743 .409322$ $-2.5561341 .5396731 .675473$ $\begin{array}{lll}-2.8708002 .868485 & 2.791408\end{array}$ $-0.3073043 .1964283 .748392$ $-0.4904482 .5578064 .619855$ $-0.8712814 .1266313 .882476$ 0.7580303 .4424343 .730370 $2.0249013 .839870-1.636656$ $2.5812632 .959993-1.295790$ $1.5563963 .584084-3.074266$ $2.4232343 .453450-3.731906$ $0.979094 \quad 4.437025-3.451667$ $\begin{array}{llll}0.942801 & 2.684144 & -3.109147\end{array}$ 
$3.179506-3.958150-2.623712$

$5.705655-2.955442-2.522510$

$6.155758-0.506867-1.441315$

Int2b

$\mathrm{Ga}$

$\mathrm{Ga}$

$1995620.015523-0.308431$

$-1.162371-0.2528050 .349528$

$2.0607731 .503743 \quad 0.633144$

$1.455621-1.1329551 .468798$

$-1.444877-1.2939492 .010257$

$\begin{array}{lll}-3.054450 & 0.159937 & 0.193734\end{array}$

$\begin{array}{llll}1.485428 & 2.802794 & 0.457676\end{array}$

$\begin{array}{llll}0.525396 & 3.206237 & 1.411941\end{array}$

$-0.0001434 .4980141 .328324$

$-0.7190754 .8339312 .072003$

$\begin{array}{llll}0.408621 & 5.370350 & 0.326247\end{array}$

$\begin{array}{llll}0.003161 & 6.377072 & 0.280216\end{array}$

$\begin{array}{llll}1.332099 & 4.944743 & -0.621537\end{array}$

$\begin{array}{llll}1.640956 & 5.626027 & -1.410653\end{array}$

$\begin{array}{llll}1.880003 & 3.660426 & -0.581855\end{array}$

$\begin{array}{llll}0.143326 & 2.315141 & 2.590770\end{array}$

$\begin{array}{llll}0.494881 & 1.294409 & 2.399372\end{array}$

$\begin{array}{lll}-1.368198 & 2.2492972 .821411\end{array}$

$-1.5956331 .5143623 .600470$

$-1.9126591 .9528061 .917048$

$-1.7766463 .2136423 .142997$

$\begin{array}{llll}0.837179 & 2.787513 & 3.876740\end{array}$

$\begin{array}{llll}0.553218 & 2.150130 & 4.721747\end{array}$

0.5483163 .8178784 .114147

$\begin{array}{llll}1.925655 & 2.751288 & 3.779823\end{array}$

$2.916430 \quad 3.255012-1.614697$

$3.2509762 .241222-1.369445$

$2.313997 \quad 3.232916-3.024793$

$\begin{array}{llll}3.080475 & 2.946137 & -3.753688\end{array}$

$\begin{array}{lll}1.944953 & 4.227310 & -3.304697\end{array}$

$\begin{array}{lll}1.495578 & 2.514355 & -3.066320\end{array}$

$4.1308234 .194002-1.578400$

$4.552820 \quad 4.287902-0.572995$

$3.8568445 .200252-1.915632$

$4.914523 \quad 3.824299-2.247563$

3.0034601 .3897001 .578768

$\begin{array}{llll}3.201529 & 0.250288 & 2.346053\end{array}$

$\begin{array}{llll}4.000311 & 0.284263 & 3.076621\end{array}$

$2.374455-0.9022192 .375248$

$2.643987-1.8237703 .552194$

$3.404940-2.5658013 .290140$

$1.751088-2.3544033 .883238$

$3.025979-1.2395924 .391352$

3.8952992 .5780381 .858342

$\begin{array}{llll}4.524032 & 2.752311 & 0.977947\end{array}$

$\begin{array}{llll}4.540149 & 2.392730 & 2.718826\end{array}$

3.3211013 .4916752 .031922

$0.697474-2.3737901 .369149$

$0.293106-2.3717850 .343473$

$1.533899-3.6628351 .403169$

$2.408368-3.5051750 .760818$

$1.898550-3.8870272 .409378$

$0.694395-4.8477900 .930029$

$0.364092-4.676278-0.103964$

$1.296151-5.7621810 .930022$

$-0.517912-5.0000881 .849082$

$-0.173328-5.2254342 .868102$

$-1.145537-5.8404891 .534326$

$-1.353712-3.7205761 .856221$

$-1.742740-3.5428060 .845066$

$-2.229442-3.8309372 .504883$

$-0.565465-2.4535372 .251267$

$-0.274819-2.5042133 .308216$

$-2.449481-1.0467102 .844753$

$-3.580557-0.2883542 .496013$

$-4.324831-0.1453323 .268296$

$-3.9164690 .154096 \quad 1.211786$

$\begin{array}{lll}-5.3311460 .611028 & 0.964665\end{array}$

$\begin{array}{lll}-5.845424 & -0.1009450 .309615\end{array}$

$\begin{array}{lll}-5.341436 & 1.577104 & 0.451941\end{array}$

$-5.8827400 .6882321 .901984$

$-2.415071-1.6045194 .251210$

$-2.466304-2.6961964 .266621$

$-3.248758-1.2166434 .836804$

$-1.480107-1.3121474 .740841$

$-3.5446410 .375555-1.144165$

$-3.4794651 .646436-1.730028$ 
$-3.9907041 .796428-3.023242$

-3.951835 $2.774402-3.494892$

$\begin{array}{lll}-4.529592 & 0.722139 & -3.713704\end{array}$

$\begin{array}{llll}-4.923818 & 0.861387 & -4.715574\end{array}$

$-4.541246-0.542038-3.131827$

$-4.934032-1.384549-3.692346$

$-4.040952-0.740731-1.847006$

$-2.8446142 .837624-1.037527$

$-2.5431052 .537228-0.028112$

$-1.5828963 .276162-1.791220$

$-0.8230112 .488441-1.817645$

$-1.1491084 .152018-1.303087$

$-1.8317213 .552487-2.823048$

$-3.8222814 .009614-0.891872$

$-4.7382513 .721895-0.364444$

$-4.1106504 .407435-1.870937$

$-3.3441804 .820161-0.332321$

$-3.928396-2.141101-1.262426$

$-3.960331-2.066670-0.167943$

$-2.563297-2.726896-1.647831$

$-1.750366-2.134083-1.214448$

$-2.421168-2.711436-2.732999$

$-2.453973-3.758909-1.296279$

$-5.058858-3.076978-1.694288$

$-4.999345-4.015554-1.135024$

$-4.991024-3.329364-2.757385$

$-6.041502-2.630073-1.513122$

$4.466962-2.176318-1.955948$

$3.917744-3.303991-2.548252$

$2.539658-3.449770-2.517748$

$1.748119-2.481691-1.914205$

$2.270510-1.358183-1.306850$

$3.645505-1.231441-1.354796$

$4.547777-4.048166-3.019525$

$0.408473-2.699617-1.912478$

$0.7209701 .066463-1.751603$

$4.236315-0.166887-0.772866$

$1.966101-4.530663-3.063649$

$5.793618-2.000594-1.962820$

$-1.2190190 .396835-0.427800$ $1.076697-0.0348150 .371283$

$-2.537716-0.7172420 .504501$

$-1.2000671 .6458431 .306229$

$\begin{array}{lll}1.582377 & 0.947680 & 2.016702\end{array}$

$2.760374-1.0076550 .345884$

$-2.380466-2.1359870 .399418$

$-1.641424-2.7708121 .421978$

$-1.535840-4.1638251 .406510$

$-0.994092-4.6696422 .202469$

$-2.140926-4.9133790 .404421$

$-2.063120-5.9968610 .410643$

$-2.837798-4.268487-0.610837$

$-3.299549-4.857269-1.399758$

$-2.965361-2.877851-0.639673$

$-1.061263-1.9869802 .596094$

$-1.076808-0.9172882 .356498$

$0.386903-2.3705152 .908894$

$0.791203-1.7067203 .680062$

$1.035085-2.2891232 .028438$

$0.469102-3.3992133 .276767$

$-1.922999-2.1712113 .853720$

$-1.495817-1.6147684 .695658$

$-1.973648-3.2295004 .134689$

$-2.942836-1.8099033 .695800$

$-3.771327-2.221677-1.746706$

$-3.789140-1.144076-1.551031$

$-3.119388-2.449498-3.115933$

$-3.720142-1.973964-3.899548$

$-3.062150-3.520742-3.345211$

$-2.117828-2.020052-3.124245$

$-5.216179-2.741303-1.762372$

$-5.699867-2.653341-0.784604$

$-5.248224-3.798129-2.051237$

$-5.810914-2.182397-2.492287$

$-3.444566-0.2829281 .390770$

$-3.3227400 .8930032 .116853$

$-4.1274871 .1326272 .800980$

$-2.1865521 .7427052 .163361$

$\begin{array}{lll}-2.2195632 .752456 & 3.297847\end{array}$

$-2.7052433 .6777562 .971899$
$-1.2246983 .0024723 .666906$ -2.8009002 .347590
4.128259 $-4.668317-1.1329021 .649129$ $-5.272780-1.1556700 .735546$ $-5.271144-0.7189402 .459048$ $-4.408196-2.1667401 .890271$ $-0.0969442 .5941281 .217226$ $\begin{array}{llll}0.335915 & 2.424785 & 0.217622\end{array}$ $-0.5030764 .0754781 .166275$ -1.352387 4.1606670 .478158 $\begin{array}{lll}-0.829106 & 4.443611 & 2.143060\end{array}$ $\begin{array}{llll}0.677983 & 4.929323 & 0.709637\end{array}$ $\begin{array}{llll}0.988566 & 4.620532 & -0.298212\end{array}$ 0.3832985 .9818230 .648693 $\begin{array}{llll}1.834761 & 4.749611 & 1.693143\end{array}$ $\begin{array}{lll}1.527604 & 5.114568 & 2.683343\end{array}$ $2.7024325 .345856 \quad 1.391517$ $2.2406513 .279072 \quad 1.786665$ $\begin{array}{llll}2.605200 & 2.946295 & 0.805993\end{array}$ $\begin{array}{llll}3.076276 & 3.149155 & 2.482930\end{array}$ $\begin{array}{lll}1.086582 & 2.328410 & 2.170404\end{array}$ $\begin{array}{llll}0.774266 & 2.512877 & 3.206075\end{array}$ 2.4202820 .4467452 .918383 $3.281946-0.6342552 .662461$ $3.907600-0.9600843 .482981$ $3.531003-1.2159921 .414479$ $4.750324-2.0897331 .268444$ $5.491049-1.5949510 .630426$ $4.493291-3.0344530 .780946$ $5.201858-2.2903622 .240403$ $2.487956 \quad 1.053352 \quad 4.303385$ $\begin{array}{llll}2.871371 & 2.076825 & 4.290438\end{array}$ $3.131678 \quad 0.457270 \quad 4.950504$ $1.4850421 .083161 \quad 4.742245$ $3.227935-1.422193-0.952636$ $2.807168-2.638653-1.505931$ $3.308777-2.993212-2.762441$ $2.996311-3.933517-3.208096$ $4.180904-2.163854-3.450063$ $4.560231-2.459658-4.423297$ $4.550726-0.938935-2.902937$ $5.208217-0.280604-3.462069$ $4.075025-0.541758-1.655293$ $1.810910-3.552444-0.817455$ $1.566814-3.1297520 .163201$ $0.512328-3.628798-1.629838$ $0.025756-2.652411-1.722263$ $-0.185952-4.313283-1.142465$ $0.714337-4.012565-2.637243$ $2.383391-4.954839-0.580102$ $3.317434-4.929886-0.008370$ $2.584977-5.464719-1.528464$ $1.657929-5.559431-0.026126$ $\begin{array}{llll}4.370656 & 0.850193 & -1.116588\end{array}$ $\begin{array}{llll}4.322298 & 0.818550 & -0.020688\end{array}$ $3.2748601 .808808-1.601331$ $\begin{array}{llll}2.297843 & 1.513571 & -1.203779\end{array}$ $3.1904421 .789961-2.692461$ $3.4718402 .839051-1.284484$ $\begin{array}{llll}5.755629 & 1.373714 & -1.501792\end{array}$ $\begin{array}{llll}5.961026 & 2.308432 & -0.971343\end{array}$ $\begin{array}{llll}5.823332 & 1.588616 & -2.573097\end{array}$ $\begin{array}{llll}6.541369 & 0.654743 & -1.249103\end{array}$ $-3.5748773 .418889-2.330219$ -2.652823 $4.292567-2.907073$ $-1.3023274 .012740-2.797150$ $-0.8802132 .871305-2.123800$ $-1.7488761 .985548-1.525222$ $-3.0909332 .303352-1.665547$ $0.4597272 .671471-2.048586$ $-1.000747-0.831364-1.793377$ $-3.9918141 .471617-1.088074$ $-0.3879044 .840336-3.326867$ $-2.9645515 .184664-3.438432$ $\begin{array}{llll}-4.642079 & 3.596777 & -2.394930\end{array}$

$\begin{array}{llll}1.550944 & 0.091356 & -0.057428\end{array}$ $-2.454987-0.995112-0.992241$ $1.1392431 .961993-0.691698$ $0.569702-0.641312-1.686417$ $-1.091711-1.7063680 .370741$ 
$-3.2572701 .484466-2.799019$ $-5.802451-1.9729580 .898294$ $-5.018599-1.9328921 .662752$ $-5.322000-2.948757-0.184584$ $-4.404808-2.566792-0.641266$ $-6.071815-3.041200-0.977589$ $-5.122891-3.9414550 .232174$ $-7.073294-2.4928541 .571086$ $-7.471780-1.7731102 .293714$ $-6.857349-3.4262242 .099632$ $-7.858054-2.7097680 .838815$ $-0.806574-4.3642040 .686796$ $4.690923-0.4961361 .338296$ $3.832931-1.3747671 .982413$ $3.617887-2.6414311 .462491$ $4.267685-3.0341850 .301761$ $5.127007-2.156947-0.345326$ $5.354375-0.8937520 .186246$ $4.031018-4.236970-0.219931$ $2.738078-3.4624432 .036251$ $3.143830-0.9672053 .042046$ $\begin{array}{llll}4.815303 & 0.747369 & 1.784046\end{array}$ $6.185322-0.061487-0.432957$ $5.746859-2.534489-1.458004$

Int3b

$-1.634364-0.0012790 .093007$ $2.408207-0.9325481 .001963$ $\begin{array}{llll}-1.289761 & 1.896075 & 0.693511\end{array}$ $-0.614884-0.6662131 .729653$ $1.053654-1.721255-0.325933$ $3.443574-0.161293-0.553149$ $-1.8589242 .857679-0.197762$ $-1.3133753 .046570-1.476359$ $-1.9001094 .003357-2.313848$ $-1.4795724 .171264-3.302020$ $-3.0052914 .732353-1.909310$ $-3.4418225 .474999-2.570103$ $-3.5712314 .493289-0.658746$ $-4.4561145 .047108-0.362964$ $\begin{array}{llll}-3.026119 & 3.549036 & 0.205853\end{array}$ $-0.1350052 .241117-2.000845$ $0.2052561 .540089-1.227601$ $1.0618143 .136527-2.335265$ $1.360173 \quad 3.738412-1.471150$ $0.8315753 .821215-3.159324$ $1.9168372 .520869-2.637650$ $-0.5589191 .422466-3.226844$ $-1.3894110 .750327-2.986392$ $0.2827480 .824369-3.587398$ $-0.8807542 .081353-4.041125$ $-3.7052283 .1952591 .523521$ $\begin{array}{lll}-2.935392 & 3.079497 & 2.295492\end{array}$ $-4.6943894 .2494312 .018252$ $-4.2338485 .2406932 .083798$ $-5.0589063 .9753353 .012721$ $-5.5664924 .3201461 .359578$ $-4.3990181 .8361431 .368110$ $-3.6641631 .1034821 .024620$ $\begin{array}{lll}-5.190758 & 1.894227 & 0.615680\end{array}$ $-4.8322641 .4896292 .311903$ $\begin{array}{lll}-0.6085962 .316130 & 1.758413\end{array}$ $\begin{array}{lll}-0.175387 & 1.4597812 .772738\end{array}$ $0.282522 \quad 1.923675 \quad 3.637288$ $\begin{array}{lll}-0.2174420 .052780 & 2.774096\end{array}$ $0.318214-0.6172854 .020624$ $1.343708-0.9631343 .837635$ $-0.288939-1.4768244 .314853$ $\begin{array}{lll}0.339172 & 0.092198 & 4.848898\end{array}$ $-0.2826613 .7866681 .895217$ $0.332184 \quad 4.1008531 .043072$ $\begin{array}{llll}0.264806 & 3.980975 & 2.818307\end{array}$ $-1.1818904 .4083811 .879197$ $0.490368-4.0500750 .395657$ $1.342149-4.1115221 .089387$ $-0.669422-4.9221800 .885147$ $-1.476382-4.8868690 .141622$ 
$0.271303 \quad 2.240901 \quad 1.989506$ $-0.0813061 .5444151 .217809$ $-0.9127623 .1504252 .331431$ $-1.2079673 .7577931 .470076$ $-0.6701673 .8304593 .155833$ $\begin{array}{lll}-1.773852 & 2.5448512 .636835\end{array}$ 0.6924471 .4163393 .212513 $\begin{array}{llll}1.510863 & 0.731477 \quad 2.966455\end{array}$ $\begin{array}{lll}-0.155618 & 0.830956 & 3.578934\end{array}$ $\begin{array}{lll}1.029605 & 2.070590 \quad 4.024346\end{array}$ $3.8303773 .161447-1.555158$ $3.0563143 .060386-2.324991$ $4.836734 \quad 4.198708-2.050372$ $4.3928955 .197578-2.113509$ $5.1945373 .920491-3.046218$ $\begin{array}{llll}5.710866 & 4.254537 & -1.392806\end{array}$ $\begin{array}{llll}4.497672 & 1.787808 & -1.406149\end{array}$ $3.752037 \quad 1.069275-1.059630$ $5.2905891 .836035-0.651479$ $\begin{array}{llll}4.919662 & 1.436639 & -2.353907\end{array}$ $0.7234752 .312983-1.774460$ $0.2787021 .461102-2.787212$ $-0.1815161 .929291-3.648255$ $\begin{array}{llll}0.310335 & 0.053179 & -2.791020\end{array}$ $-0.236534-0.609816-4.036580$ $-1.263755-0.948043-3.848716$ $0.361942-1.473615-4.335895$ $\begin{array}{llll}-0.256413 & 0.101637 & -4.863211\end{array}$ $0.4084253 .786309-1.908250$ $-0.2000184 .104232-1.052891$ $-0.1419513 .986164-2.828463$ $\begin{array}{llll}1.312378 & 4.401292 & -1.895471\end{array}$ $-0.404136-4.056239-0.424342$ $-1.260044-4.110585-1.113585$ $0.749325-4.930793-0.924206$ $1.561042-4.901478-0.185682$ $0.427647-5.974419-1.005153$ $1.283524-4.435920-2.271918$ $2.162539-5.018971-2.565544$ $0.518311-4.595862-3.044192$ $1.637375-2.946879-2.224339$ $1.959194-2.586133-3.207937$ $2.480533-2.779898-1.541381$ $0.445051-2.113225-1.739318$ $-0.409175-2.330962-2.392259$ $0.055674-2.596285-0.316943$ $0.974610-2.5836980 .283690$ $-0.957273-1.6142321 .637794$ $-1.881174-0.8257622 .353241$ $-1.740815-0.7864723 .425462$ $-2.987115-0.1389031 .852473$

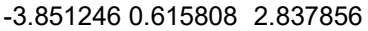
$\begin{array}{lll}-4.818760 & 0.1186852 .969039\end{array}$ $\begin{array}{lll}-4.062663 & 1.622890 & 2.464809\end{array}$ $-3.3602170 .6875633 .809981$ $0.086321-2.3111812 .485505$ $1.058611-1.8186302 .362363$ $0.211476-3.3618132 .214878$ $-0.185515-2.2561023 .540292$ $\begin{array}{llll}-4.587283 & 0.395984 & 0.172097\end{array}$ $-4.6533701 .635699-0.483019$ $\begin{array}{lll}-5.9061572 .102579 & -0.896835\end{array}$ $-5.9735443 .060969-1.405191$ $-7.0584441 .367019-0.672633$ $-8.0218841 .749018-0.996120$ $-6.9754220 .128756-0.041712$ $-7.880616-0.4491890 .115374$ $-5.749563-0.3826940 .376308$ $\begin{array}{lll}-3.422620 & 2.483089 & -0.766947\end{array}$ $-2.5455151 .995097-0.322520$ $-3.5503843 .874699-0.134513$ $-3.6972523 .8122830 .948689$ $\begin{array}{lll}-4.397130 & 4.425623 & -0.557740\end{array}$ $-2.6471174 .465240-0.324063$ $-3.1838232 .605093-2.277565$ $-2.2915503 .209164-2.471532$ $-4.0357913 .092490-2.765405$ $-3.0397191 .621757-2.736788$ $-5.645359-1.7870220 .957259$ $-4.838910-1.7982021 .698727$ $-5.240428-2.760205-0.158392$ $-4.320719-2.409144-0.634458$ 
$4.856263-0.7976711 .172033$ $3.984883-1.6627641 .821429$ $3.733959-2.9115191 .280043$ $4.364253-3.2863350 .099538$ $5.241993-2.431546-0.542613$ $5.493176-1.1731690 .003430$ $5.710673-2.755772-1.464095$ $4.067102-4.483765-0.421364$ $2.842743-3.7235681 .853170$ $3.332185-1.2672892 .909767$ $\begin{array}{llll}5.005910 & 0.429051 & 1.672970\end{array}$ $6.154274-0.467203-0.484734$

Int4a

Ga

$-2.553424-0.346597-0.354930$ $1.777648-0.361460-0.202129$ $-3.816483-0.6362851 .067565$ $-1.253931-1.6474760 .234670$ $0.387846-0.255139-1.701041$ $2.259514 \quad 1.539245-0.554316$ $-5.208026-0.5416670 .747437$ $\begin{array}{llll}-5.925398 & 0.638284 & 0.998100\end{array}$ $\begin{array}{llll}-7.289109 & 0.657464 & 0.697497\end{array}$ $\begin{array}{llll}-7.862555 & 1.561857 & 0.883673\end{array}$ $-7.918030-0.4546920 .153212$ $-8.980666-0.424587-0.067279$ $7.180470-1.598859-0.129181$ $-7.672942-2.457279-0.578047$ $-5.814476-1.6605320 .145858$ $-5.2403621 .9000531 .492414$ $-4.2001401 .6543871 .736680$

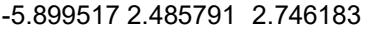
$-5.9337931 .7655813 .569223$ $-6.9278732 .8003962 .539208$ $-5.3474023 .3673953 .087091$ $\begin{array}{lll}-5.227259 & 2.948127 & 0.369741\end{array}$ $-4.8874462 .517602-0.575413$ $-4.5720433 .7866220 .632099$ $\begin{array}{llll}-6.235634 & 3.345076 & 0.205896\end{array}$ $-5.020748-2.914350-0.186349$ $-3.958159-2.680960-0.081056$ $-5.347628-4.0457560 .794872$ $-5.118862-3.7591421 .826895$ $-4.764619-4.9414330 .555207$ $-6.410140-4.3093520 .744502$ $-5.237977-3.359123-1.634983$ $-4.969461-2.554388-2.322798$ $-6.276168-3.657121-1.817335$ $-4.606462-4.227989-1.852570$ $-3.410325-1.0641122 .259832$ $-2.112580-1.5328862 .498338$ $-1.879373-1.7891323 .522801$ $-1.174329-1.9474321 .530973$ $-0.060393-2.8359732 .026467$ $0.908357-2.3825231 .796750$ $0.089048-3.8198571 .547679$ $-0.133973-2.9759873 .104737$ $-4.404578-1.1016483 .392009$ $-4.810458-0.1013573 .571422$ $-3.941277-1.4702714 .307220$ $-5.251565-1.7454873 .134656$ $0.744120-2.377914-2.932787$ $1.680875-2.574733-2.388849$ $0.087185-3.696085-3.348813$ $-0.797867-3.477341-3.961514$ $0.772497-4.278787-3.972964$ $-0.338738-4.508190-2.122394$ $-0.866307-5.416860-2.430319$ $0.557929-4.826674-1.572468$ $-1.220051-3.674226-1.188781$ $-1.502174-4.249629-0.298321$ $2.152805-3.381267-1.691782$ $-0.480139-2.400001-0.758983$ $0.480732-2.691339-0.323888$ $-0.191281-1.562943-2.024894$

Int4b
$-1.150330-1.435206-2.539740$ $\begin{array}{llll}0.133345 & 0.788610 & -2.481987\end{array}$ $\begin{array}{llll}0.688126 & 2.070078 & -2.273695\end{array}$ $0.3101342 .863456-2.906318$ $\begin{array}{llll}1.669829 & 2.428538 & -1.345387\end{array}$ $2.0612743 .882817-1.226904$ $3.1394534 .021989-1.345223$ $\begin{array}{llll}1.802243 & 4.236649 & -0.221425\end{array}$ $\begin{array}{llll}1.535566 & 4.499145 & -1.958532\end{array}$ $-0.8315270 .662165-3.643693$ $\begin{array}{llll}-1.825238 & 0.341925 & -3.310858\end{array}$ $-0.474462-0.079826-4.365637$ $-0.9357701 .618311-4.157972$ $\begin{array}{llll}3.514350 & 1.782910 & 0.078812\end{array}$ $3.5958251 .840974 \quad 1.479742$ $\begin{array}{llll}4.862386 & 1.900561 & 2.070738\end{array}$ $4.941497 \quad 1.9586333 .153402$ $6.0096601 .886142 \quad 1.296596$ $\begin{array}{llll}6.986568 & 1.930790 & 1.767082\end{array}$ $\begin{array}{llll}5.914005 & 1.779494 & -0.090015\end{array}$ $6.8276061 .735079-0.671699$ $\begin{array}{llll}4.676130 & 1.702038 & -0.727053\end{array}$ 2.3588521 .9111822 .358134 $1.480948 \quad 1.9236461 .709061$ $2.349424 \quad 3.218033 \quad 3.162821$ 2.4597934 .0881422 .508907 $\begin{array}{llll}3.166737 & 3.236721 & 3.892171\end{array}$ $\begin{array}{llll}1.406739 & 3.318564 & 3.709125\end{array}$ $\begin{array}{llll}2.222017 & 0.714166 & 3.308551\end{array}$ $\begin{array}{lll}1.331866 & 0.841369 & 3.934182\end{array}$ $\begin{array}{llll}3.094136 & 0.629760 & 3.967390\end{array}$ $2.116021-0.2295562 .763773$ $\begin{array}{llll}4.573826 & 1.569460 & -2.250816\end{array}$ $3.9375732 .388498-2.611936$ $\begin{array}{llll}3.914620 & 0.255868 & -2.727969\end{array}$ $\begin{array}{llll}2.823262 & 0.314020 & -2.745947\end{array}$ $4.212884-0.594901-2.109784$ $\begin{array}{llll}4.236394 & 0.035448 & -3.751403\end{array}$ $\begin{array}{llll}5.930644 & 1.726191 & -2.942962\end{array}$ $6.4339042 .654611-2.657589$ $\begin{array}{llll}5.788807 & 1.737471 & -4.027202\end{array}$ $\begin{array}{lllll}6.590730 & 0.885111 & -2.703263\end{array}$ $1.012632-1.776074-3.808613$ $-2.0457781 .558129-0.412223$ $-2.4624552 .458466-1.375273$ $\begin{array}{lll}-1.214229 & 2.058650 & 0.573658\end{array}$ $-2.0627653 .788437-1.391576$ $-0.7911123 .3784090 .605683$ $-1.2145004 .244822-0.394295$ $-3.264088-0.926966-1.896564$ $\begin{array}{llll}-3.3007732 .072166 & -2.349969\end{array}$ $0.027483 \quad 3.8363381 .555437$ $-0.7844151 .2357191 .554366$ $5.800949-1.4000041 .164784$ $4.921247-1.7132512 .193055$ $3.825835-2.5286381 .943518$ $3.590041-2.9973760 .661544$ $4.432216-2.633745-0.375695$ $5.533362-1.827649-0.129045$ $2.506356-3.7276420 .397832$ $2.970647-2.8094312 .924197$ $6.342137-1.478543-1.126277$ $4.124837-2.987238-1.622922$ $-2.4634264 .620831-2.351373$ $\begin{array}{lll}-0.786045 & 5.504397 & -0.399999\end{array}$ $6.918478-0.7327501 .424002$ $5.152878-1.2685123 .423346$

$\begin{array}{llll}-2.416054 & 0.216619 & 0.391867\end{array}$ $\begin{array}{llll}1.893951 & 0.317761 & 0.189829\end{array}$ $-3.6891190 .493515-1.026420$ $-1.1424501 .543610-0.195754$ $\begin{array}{llll}0.530109 & 0.159525 & 1.709039\end{array}$ $2.439762-1.5744470 .527223$ $-5.0787520 .385934-0.702230$ $-5.787417-0.799499-0.951628$ $-7.150551-0.829186-0.648835$ $-7.717107-1.738089-0.834318$ $\begin{array}{llll}-7.787441 & 0.277847 & -0.103622\end{array}$ $\begin{array}{lll}-8.849503 & 0.239422 & 0.118456\end{array}$ $\begin{array}{lll}-7.058324 & 1.427740 & 0.177613\end{array}$ 
$4.056018-0.2671322 .681238$ $2.972314-0.3956562 .734732$ $4.281034 \quad 0.600834 \quad 2.057314$ $4.396695-0.0270653 .694145$ $6.153435-1.6289972 .855389$ $6.688960-2.5424722 .580506$ $6.032477-1.6239713 .942118$ $6.773811-0.7671782 .585477$ $\begin{array}{llll}1.134652 & 1.662172 & 3.838822\end{array}$ $-1.890518-1.6836120 .439055$ $-2.316633-2.5896891 .392026$ $-1.057227-2.178651-0.548604$ $-1.928940-3.9243511 .377356$ $-0.655977-3.506104-0.585584$ $-1.092162-4.3984760 .382142$ $-0.783699-5.4368120 .357578$ $-3.1337260 .7907241 .933156$ $-3.156029-2.2051222 .368963$ $-2.372043-4.7494902 .330216$ $0.161912-3.935788-1.556217$ $-0.614226-1.343362-1.516031$ $5.4811271 .364601-1.453001$ $\begin{array}{llll}4.610507 & 1.878652 & -2.398680\end{array}$ $3.6376242 .808916-2.057042$ $3.5013093 .183656-0.730089$ $\begin{array}{llll}4.358600 & 2.676897 & 0.232744\end{array}$ $5.347077 \quad 1.777886-0.138290$ $\begin{array}{llll}6.235700 & 0.640531 & -1.729531\end{array}$ $2.4914743 .969846 \quad-0.354731$ $2.8064923 .292249-2.980493$ $4.7030821 .499688-3.673648$ $\begin{array}{llll}6.164284 & 1.304244 & 0.806198\end{array}$ $\begin{array}{llll}4.150588 & 2.976597 & 1.516332\end{array}$

$2.3738510 .128135-0.443261$ $\begin{array}{lll}-1.928174 & 0.318045 & -0.071535\end{array}$ $\begin{array}{llll}3.687787 & 0.229007 & 0.964066\end{array}$ $\begin{array}{llll}1.149388 & 1.412053 & 0.324279\end{array}$ $\begin{array}{llll}-0.628830 & 0.283042 & -1.654124\end{array}$ $-2.553212-1.510959-0.589351$ $\begin{array}{llll}5.066991 & 0.137428 & 0.595453\end{array}$ $5.760977-1.0783140 .695836$ $7.115950-1.0943820 .357544$ $7.671044-2.0262800 .428412$ $\begin{array}{llll}7.758714 & 0.056558 & -0.079268\end{array}$ $\begin{array}{llll}8.814582 & 0.027515 & -0.330625\end{array}$ $\begin{array}{llll}7.042922 & 1.240862 & -0.214823\end{array}$ $\begin{array}{llll}7.545334 & 2.132363 & -0.580456\end{array}$ $\begin{array}{llll}5.686251 & 1.301882 & 0.102866\end{array}$ $5.056704-2.3711321 .067513$ $4.029905-2.1303961 .366573$ $5.732989-3.1076192 .229230$ $5.808483-2.4842583 .125389$ $6.746790-3.4216561 .958735$ $5.166498-4.0082912 .486712$ $4.985923-3.286721-0.163455$ $4.631434-2.744677-1.043418$ $4.313378-4.1317930 .021544$ $5.979509-3.685695-0.399067$ $4.9146452 .601375-0.066116$ $\begin{array}{llll}3.849623 & 2.376943 & 0.031912\end{array}$ 5.2828713 .6041591 .033169 $\begin{array}{llll}5.063812 & 3.204453 & 2.029119\end{array}$ $\begin{array}{llll}4.716517 & 4.533535 & 0.909744\end{array}$ $\begin{array}{llll}6.350269 & 3.849748 & 0.994059\end{array}$ $5.1143873 .207329-1.457458$ $\begin{array}{llll}4.815258 & 2.492581 & -2.227313\end{array}$ $6.154843 \quad 3.505420-1.626830$ $\begin{array}{llll}4.496761 & 4.106932 & -1.559700\end{array}$ 3.3290110 .5369542 .207936 $2.0570401 .020148 \quad 2.535477$ 1.8596021 .1713673 .588034 $\begin{array}{llll}1.113416 & 1.574823 & 1.645626\end{array}$ $\begin{array}{llll}0.047053 & 2.443140 & 2.263615\end{array}$ $-0.9382892 .0492301 .993458$ 0.1037363 .4749981 .903157 $0.1368662 .451443 \quad 3.349455$ $\begin{array}{llll}4.353455 & 0.416533 & 3.307443\end{array}$ $4.727838-0.6101873 .364017$ $\begin{array}{llll}3.927228 & 0.696681 & 4.270850\end{array}$ $\begin{array}{llll}5.215937 & 1.057027 & 3.097778\end{array}$ 
$\begin{array}{llll}0.448095 & 3.178416 & -0.975355\end{array}$ $\begin{array}{lll}-3.7042072 .622688 & 1.173500\end{array}$ $-4.6848061 .404077-1.048857$ $\begin{array}{lll}-3.069979 & 0.968227 & -3.184450\end{array}$

Int5b Ga

$\mathrm{N}$

C

H

C

C

C

$\mathrm{H}$

$\mathrm{H}$

H

C

C

C

C

$\mathrm{H}$

$\mathrm{H}$

C

C

H

$\mathrm{H}$

C

$\mathrm{H}$

C

$\mathrm{H}$

$\mathrm{H}$

C

$\mathrm{H}$

$\mathrm{H}$

C

$\mathrm{H}$

$\mathrm{H}$

C

$\mathrm{H}$

$\mathrm{H}$

C

$\mathrm{H}$
$\mathrm{H}$

$\mathrm{H}$

C

$\mathrm{H}$

$\mathrm{H}$

C

$\mathrm{H}$

$\mathrm{H}$

C

$\mathrm{H}$

$\mathrm{H}$

C

H

$\mathrm{H}$

C

$\mathrm{H}$
$\mathrm{H}$

$\mathrm{H}$

C

C $\begin{array}{llll}0.381854 & 0.126494 & -0.126574\end{array}$ $\begin{array}{lll}-0.502542 & 1.597497 & 0.958012\end{array}$ $\begin{array}{lll}2.136065 & 0.807225 & 0.647066\end{array}$ $0.0142972 .532179 \quad 1.745788$ 2.3897221 .8154341 .470194 $\begin{array}{llll}1.387974 & 2.648704 & 1.990916\end{array}$ $\begin{array}{llll}1.704918 & 3.446523 & 2.649778\end{array}$ -1.912044 1.4909640 .756337 $\begin{array}{lll}-2.663088 & 0.671262 & 1.619297\end{array}$ $-2.4762062 .040392-0.409398$ -4.0041890.437992 1.310646

$-3.8243241 .789573-0.672443$ $\begin{array}{lll}-4.586490 & 1.000258 & 0.180125\end{array}$ $-4.599060-0.2023541 .956309$ $-4.2811872 .209974-1.565436$ $\begin{array}{llll}-5.628315 & 0.801819 & -0.049160\end{array}$ $3.197998-0.0142590 .158205$ $\begin{array}{llll}3.798343 & 0.301625 & -1.073635\end{array}$ $3.513794-1.2022170 .841392$ $4.796737-0.543835-1.559477$ $4.518171-2.0186760 .319099$ $5.170249-1.686495-0.862263$ $5.276235-0.312533-2.507398$ $4.780291-2.9380070 .836911$ $5.949491-2.332915-1.254134$ $\begin{array}{llll}-2.026597 & 0.027562 & 2.843277\end{array}$ -1.070113 0.5273123 .030665 $-1.6573362 .892874-1.368675$ $-0.6458122 .997606-0.960607$ $\begin{array}{llll}3.332181 & 1.485583 & -1.906443\end{array}$ $2.5832812 .043068-1.333246$ $2.741638-1.6411892 .076231$ $2.028270-0.8527442 .341625$ 3.8189092 .0889981 .874035 $\begin{array}{llll}4.249558 & 1.209782 & 2.363967\end{array}$ $\begin{array}{llll}4.432815 & 2.285099 & 0.989063\end{array}$ $\begin{array}{lll}3.882876 & 2.942365 & 2.550017\end{array}$ -0.917792 3.5091992 .420674 $-1.5998592 .9775533 .093683$ $\begin{array}{lll}-0.365042 & 4.2546112 .993687\end{array}$ $-1.5411254 .0159321 .676973$ $\begin{array}{llll}2.664995 & 1.004343 & -3.203244\end{array}$ $\begin{array}{llll}3.393026 & 0.494552 & -3.844570\end{array}$ $\begin{array}{llll}1.848005 & 0.302643 & -3.004528\end{array}$ $\begin{array}{llll}2.262733 & 1.856551 & -3.761890\end{array}$ $\begin{array}{llll}4.479415 & 2.451040 & -2.226275\end{array}$ $\begin{array}{llll}4.962866 & 2.819477 & -1.315955\end{array}$ $\begin{array}{llll}5.246860 & 1.964200 & -2.837797\end{array}$ $\begin{array}{llll}4.106529 & 3.313779 & -2.787728\end{array}$ $1.941566-2.9194501 .787116$ $1.287326-2.8015490 .917446$ $2.616135-3.7586461 .582169$ $1.323570-3.1864822 .651287$ $3.662863-1.8515333 .283591$ $4.381333-2.6569683 .096397$ $4.230775-0.9462173 .520445$

$3.077365-2.1279074 .166404$ $-1.5272542 .239233-2.750983$ $-1.0350121 .262001-2.693378$ $-25140482.099653-3208878$ $-0.9373572 .878455-3.416791$ $-2.2533754 .300294-1.502724$ -3.248659 4.264621 -1.959013 $-2.3519564 .788854-0.528330$ $\begin{array}{lll}-1.617798 & 4.926002 & -2.137547\end{array}$ $-2.8939470 .1868064 .096330$ $-3.819891-0.3925214 .018882$ $-2.354295-0.1758234 .976753$ -3.166864 1.2334384 .266040 $-1.714103-1.4527412 .587777$ $-2.620479-2.0045402 .316938$ $-0.996265-1.5704551 .767909$ $-1.282059-1.9151993 .482149$ $-1.682188-2.939245-0.567255$ $-0.800581-2.586445-1.576943$ $-2.981477-2.446691-0.565401$
$-3.390583-1.608448-1.593203$ $-1.221756-1.712904-2.568094$ $-2.515142-1.221109-2.595448$ $-1.267549-3.7224010 .425943$ $-4.661427-1.205283-1.617719$ $-3.830695-2.8013100 .399764$ $-2.835488-0.542114-3.375538$ $0.459399-3.009823-1.534095$ $-0.329504-1.302041-3.470568$

Int5c $\mathrm{Ga}$ N

$\mathrm{N}$

$\begin{array}{lll}-0.249439 & 0.106412 & -0.153910\end{array}$ $\begin{array}{lll}-1.8883160 .901505 & 0.756645\end{array}$ $\begin{array}{llll}0.825612 & 1.462741 & 0.923069\end{array}$ $-2.0070801 .8947121 .625786$ 0.4323342 .4073351 .770136 $\begin{array}{lll}-0.9089872 .625508 & 2.105379\end{array}$ $-1.1174733 .4221422 .807485$ $\begin{array}{lll}-3.041390 & 0.182042 & 0.316179\end{array}$ $-3.402986-1.0019960 .983278$ $-3.6958640 .586874-0.860769$ $-4.501303-1.7199030 .507689$ $-4.788352-0.161682-1.301105$ $-5.203085-1.296570-0.614494$ $-4.799593-2.6346121 .013986$ $-5.3105630 .139200-2.205988$ $-6.055209-1.867133-0.970812$ $2.210219 \quad 1.2612240 .639705$ $2.7623351 .838746-0.517733$ $2.941453 \quad 0.354444 \quad 1.431017$ $\begin{array}{llll}4.079687 & 1.517544 & -0.851974\end{array}$ $\begin{array}{lll}4.248697 & 0.049572 & 1.049629\end{array}$ $\begin{array}{llll}4.819554 & 0.631363 & -0.077852\end{array}$ $\begin{array}{llll}4.529217 & 1.963596 & -1.736339\end{array}$ $4.827205-0.6565401 .638578$ $\begin{array}{llll}5.841439 & 0.388256 & -0.354987\end{array}$ $-2.587730-1.5429242 .148031$ $-1.794447-0.8245392 .384342$ $\begin{array}{llll}-3.193960 & 1.760840 & -1.686938\end{array}$ $-2.3632492 .234734-1.152510$ $\begin{array}{llll}1.966966 & 2.806940 & -1.382496\end{array}$ $\begin{array}{llll}0.999167 & 2.982275 & -0.899805\end{array}$ $2.322240-0.2777692 .670694$ $\begin{array}{llll}1.434386 & 0.305698 & 2.937091\end{array}$ $\begin{array}{llll}1.478350 & 3.284597 & 2.415560\end{array}$ $2.1626672 .677157 \quad 3.018487$ $2.086420 \quad 3.777815 \quad 1.650245$ 1.0220824 .0423303 .053590 $-3.3805182 .2661432 .132020$ $-3.8499271 .4061232 .620363$ $-3.3314603 .0957722 .838148$ $-4.0310442 .5437781 .296444$ $\begin{array}{lll}1.682336 & 2.232024 & -2.776230\end{array}$ $2.6181341 .997545-3.298121$ $\begin{array}{llll}1.084324 & 1.315755 & -2.719836\end{array}$ $1.1333652 .961616-3.381371$ $2.681044 \quad 4.159516-1.502424$ $2.902393 \quad 4.585934-0.518992$ $\begin{array}{llll}3.628850 & 4.060311 & -2.042696\end{array}$ $\begin{array}{llll}2.058357 & 4.871788 & -2.053058\end{array}$ $1.846770-1.7101002 .395414$ $1.075587-1.7319111 .616888$ $2.674343-2.3415742 .058464$ $1.420243-2.1518443 .302874$ $3.277220-0.2510443 .868788$ $4.130712-0.9198883 .716157$ $3.667706 \quad 0.755665 \quad 4.050253$ $2.757259-0.5870314 .771305$ $-2.6613531 .279159-3.044262$ $\begin{array}{llll}-1.894406 & 0.506095 & -2.929968\end{array}$ $-3.4722100 .856105-3.648192$ $-2.2294152 .117919-3.601181$ $-4.2780432 .825808-1.890211$ $-5.1242572 .424447-2.458376$ $-4.6638813 .197170-0.935536$ $-3.8767153 .676658-2.450245$ $-3.439453-1.7169473 .410991$ $-4.234598-2.4535683 .252368$ $-2.820847-2.0703354 .242272$ $-3.911353-0.7763583 .712438$ $-1.919077-2.8720111 .768980$ $-2.673695-3.6459901 .588004$ 
-1.316239-2.779866 0.860054 $-1.269405-3.2173472 .580568$ $1.457197-3.056133-0.840679$ $2.748571-2.546761-0.927888$ $0.514005-2.689084-1.785038$ $0.867839-1.813356-2.801896$ $3.104580-1.683485-1.947956$ $2.153727-1.316394-2.898400$ $1.105494-3.8488620 .171809$ $-0.090070-1.401458-3.637585$ $-0.746081-3.094980-1.653842$ $2.391379-0.623826-3.696909$ $3.645479-2.923520-0.007875$ $4.117253-1.301999-1.980401$

\section{TS1a}

H
$-2.5850050 .305843-1.410753$ $-0.670997-1.805098-2.046222$ $2.028507-0.562611-2.080893$ $2.576157 \quad 1.506247 \quad-0.200096$ $-3.0758851 .509811-0.807038$ $-2.5581172 .757004-1.222515$ $-3.1930753 .899572-0.730040$ $\begin{array}{lll}-2.8604494 .881040 & -1.050508\end{array}$ $\begin{array}{lll}-4.2190523 .811067 & 0.207002\end{array}$ $\begin{array}{lll}-4.693429 & 4.716313 & 0.573484\end{array}$ $\begin{array}{lll}-4.5921152 .575500 & 0.717179\end{array}$ $-5.3348272 .5210081 .507073$ $-4.0148821 .4003040 .230854$ $\begin{array}{lll}-1.2587512 .866038 & -2.032247\end{array}$ $-0.5343282 .250403-1.479879$ $\begin{array}{lll}-0.6884024 .286440 & -2.057789\end{array}$ $\begin{array}{llll}0.324394 & 4.258557 & -2.473077\end{array}$ $-0.6299294 .727664-1.058749$ $-1.2903034 .948876-2.690702$ $-1.2664042 .319844-3.468024$ $-0.2980592 .539923-3.932029$ $-2.0414952 .802978-4.073458$ $-1.3990751 .237086-3.510914$ $\begin{array}{llll}-4.390512 & 0.039341 & 0.801889\end{array}$ $-3.533570-0.6263810 .649817$ $-4.749508-0.9341112 .692440$ -5.6123800 .5962442 .526632
-3.8603 $\begin{array}{llll}-3.860988 & 0.588886 & 2.846635\end{array}$ $-5.582221-0.5829450 .062000$ $-5.339131-0.817423-0.978567$ $\begin{array}{lll}-6.441378 & 0.098078 & 0.071418\end{array}$ $-5.882908-1.5155330 .550107$

$-3.119044-0.120358-2.559695$ $-2.582537-1.141660-3.346282$ $-3.110075-1.348016-4.268311$ $-1.387446-1.879047-3.146902$ $-0.993226-2.776326-4.300498$ $-1.288868-3.810045-4.100127$ $0.084327-2.761379-4.472888$ $-1.493410-2.451567-5.213669$ $\begin{array}{llll}-4.308698 & 0.621499 & -3.123466\end{array}$ $\begin{array}{llll}-4.791130 & 0.032047 & -3.904119\end{array}$ $-3.9735771 .569198-3.558760$ $\begin{array}{llll}-5.037629 & 0.868237 & -2.349185\end{array}$ $0.548680-2.569953-1.728810$ $0.651800-2.481206-0.640342$ $0.472415-4.082780-1.980421$ $-0.499527-4.441586-1.630792$ $0.562675-4.319533-3.045604$ $1.614160-4.781388-1.241368$ $1.510136-4.597471-0.165722$ $1.551308-5.863687-1.393749$ $2.957217-4.249108-1.742856$ $3.087900-4.528225-2.798236$ $3.785988-4.703606-1.189993$ $3.026171-2.728281-1.605690$ $2.970124-2.450092-0.543893$ $3.979635-2.340811-1.985770$ $1.863383-2.002793-2.312892$ $1.892947-2.214017-3.389170$ $\begin{array}{llll}2.821221 & 0.161980 & -2.852728\end{array}$ $\begin{array}{llll}3.310747 & 1.440083 & -2.489480\end{array}$ $3.9108601 .951452-3.230061$ $3.337497 \quad 1.968605-1.194835$ $4.3104993 .076922-0.888915$ $\begin{array}{lll}-4.667279 & 0.0869092 .306735\end{array}$ $\begin{array}{lll}5.097657 & 2.707918 & -0.223098\end{array}$ $3.809702 \quad 3.897127 \quad-0.364625$ $4.770823 \quad 3.453009-1.802915$ $3.301498-0.388021-4.177618$ $3.941679-1.263720-4.034245$ $\begin{array}{llll}3.871507 & 0.365317 & -4.721066\end{array}$ $2.452552-0.695982-4.796117$ $2.872017 \quad 1.828892 \quad 1.167487$ 2.2002092 .8915551 .791480 $2.4837353 .148648 \quad 3.133430$ $1.9708043 .959573 \quad 3.641968$ $\begin{array}{llll}3.401813 & 2.374073 & 3.831552\end{array}$ 3.6081342 .5871154 .875814 $\begin{array}{llll}4.042772 & 1.317240 & 3.198149\end{array}$ $\begin{array}{llll}4.742536 & 0.701377 & 3.756661\end{array}$ $\begin{array}{llll}3.785965 & 1.016111 & 1.859713\end{array}$ 1.1420313 .6984241 .059140 $\begin{array}{llll}1.286181 & 3.552237 & -0.019475\end{array}$ $-0.2512153 .1718931 .426356$ $-0.3767682 .1355091 .103570$ $-1.0421973 .7709330 .962381$ $-0.3965913 .1910062 .511806$ $\begin{array}{lll}1.251176 & 5.200704 & 1.336260\end{array}$ 2.2637325 .5738881 .150491 $\begin{array}{llll}0.988526 & 5.437801 & 2.372200\end{array}$ $\begin{array}{llll}0.558417 & 5.753641 & 0.693573\end{array}$ $4.463165-0.1836071 .214660$ $4.184541-0.2195760 .154205$ $3.980513-1.4841861 .872580$ $2.892392-1.5825651 .799141$ $4.260448-1.5050632 .932021$ $4.454171-2.3468891 .388715$ $5.991225-0.0730771 .281977$ $6.453989-0.9207800 .766342$ $6.343768-0.0847042 .318754$ $\begin{array}{lll}6.354020 & 0.849405 & 0.816351\end{array}$ $\begin{array}{llll}1.107390 & 0.346630 & -0.584466\end{array}$ $-1.064879-0.535420-0.590295$ $-0.867992-1.5855591 .355174$ $-0.935994-2.9464621 .654203$ $-1.327840-0.7639942 .382343$ $-1.557976-1.1877913 .677132$ $-1.126405-3.4209122 .939836$ $-1.436200-2.5399073 .972467$ $0.873698-1.2623611 .118485$ $-1.962659-0.3301594 .624800$ $-1.6218780 .5426042 .079254$ $-1.610548-2.9902855 .224625$ $-0.763328-3.8727220 .667462$ $-1.084806-4.7359543 .195888$

TS1b
$\mathrm{N}$

$\mathrm{N}$

C

C

$\mathrm{H}$

$\mathrm{C}$

$\mathrm{H}$

C

C

$\mathrm{C}$
$-2.708439-0.369373-1.146953$

$-0.670229-2.480670-1.057387$ $1.950854-1.267439-1.727892$ $2.4804011 .368249-0.784906$ $\begin{array}{lll}-3.3012590 .926649 & -0.982789\end{array}$ $\begin{array}{llll}-2.859319 & 1.999295 & -1.785357\end{array}$ $-3.5751273 .195887-1.689134$ $-3.2980034 .041332-2.309262$ $-4.6216183 .341607-0.783080$ $\begin{array}{lll}-5.162128 & 4.281868 & -0.731998\end{array}$ $-4.9350612 .3118570 .093833$ $\begin{array}{lll}-5.6964142 .463645 & 0.852723\end{array}$ $-4.2635731 .0900830 .025028$ $-1.5689551 .914436-2.610626$ $-0.8037931 .554396-1.908993$ -1.089379 3.279919 - -3.107320 $-0.0794443 .178625-3.518539$ $-1.0533494 .020097-2.302852$ $\begin{array}{lll}-1.735873 & 3.668621 & -3.902687\end{array}$ $-1.5497320 .931332-3.791038$ $-0.5884381 .026489-4.309568$ $-2.3429011 .159766-4.511912$ $-1.636160-0.112162-3.480413$ $-4.533411-0.0233721 .028018$ $-3.617098-0.6210541 .096506$ $-4.8269610 .5164312 .429652$ $-4.815971-0.3097273 .147379$ -5.8177440.982524 2.480925 $\begin{array}{llll}-5.817744 & 0.982524 & 2.480925 \\ -4.075491 & 1.244442 & 2.741953\end{array}$ $-5.659070-0.9614980 .572836$ 
$\begin{array}{lll}-2.87531800 & -1.57939200 & 2.03041400\end{array}$

$\begin{array}{lll}-3.59083300 & -2.77322400 & 2.15833900\end{array}$

$\begin{array}{lll}-3.32506000 & -3.47999000 & 2.93682100\end{array}$

$\begin{array}{lll}-4.62076700 & -3.09728400 & 1.27983800\end{array}$

$\begin{array}{lll}-5.16077100 & -4.03093900 & 1.40467800\end{array}$

$\begin{array}{lll}-4.91734000 & -2.26052800 & 0.21249900\end{array}$

$\begin{array}{lll}-5.66471600 & -2.56030800 & -0.51569300\end{array}$

$\begin{array}{lll}-4.24744300 & -1.04626500 & 0.05345600\end{array}$

$\begin{array}{lll}-1.59566300 & -1.33534000 & 2.84110500\end{array}$

$\begin{array}{lll}-0.81980100 & -1.12560100 & 2.09169600\end{array}$

$\begin{array}{lll}-1.13118900 & -2.57585100 & 3.60706400\end{array}$

$-0.12648900 \quad-2.39762900 \quad 4.00476300$

$\begin{array}{lll}-1.08808400 & -3.46180900 & 2.96712500\end{array}$

$\begin{array}{lll}-1.79113700 & -2.79622100 & 4.45422300\end{array}$

$\begin{array}{lll}-1.58287900 & -0.13584700 & 3.80094900\end{array}$

$\begin{array}{lll}-0.62979200 & -0.13254500 & 4.34278700\end{array}$

$\begin{array}{lll}-2.38793400 & -0.20876800 & 4.54091800\end{array}$

$\begin{array}{lll}-1.65349300 & 0.82456000 & 3.28573900\end{array}$

$\begin{array}{lll}-4.50478800 & -0.14870700 & -1.14913200\end{array}$

$\begin{array}{lll}-3.59252300 & 0.43574000 & -1.31422100\end{array}$

$-4.76665200-0.94863800-2.42727900$

$\begin{array}{lll}-4.74897900 & -0.27346000 & -3.28845700\end{array}$

$\begin{array}{lll}-5.75204800 & -1.42838900 & -2.40603900\end{array}$

$\begin{array}{lll}-4.00227900 & -1.71272400 & -2.58294300\end{array}$

$\begin{array}{llll}-5.64607200 & 0.84581600 & -0.89921800\end{array}$

$\begin{array}{lll}-5.39577600 & 1.57071100 & -0.11874800\end{array}$

$\begin{array}{lll}-6.55979300 & 0.31862800 & -0.60020000\end{array}$

$\begin{array}{lll}-5.86274300 & 1.40642400 & -1.81423200\end{array}$

$\begin{array}{lll}-3.23273700 & 1.61672600 & 1.67146200\end{array}$

$\begin{array}{lll}-2.64504600 & 2.87272200 & 1.83299000\end{array}$

$\begin{array}{lll}-3.17929400 & 3.55690000 & 2.47909200\end{array}$

$\begin{array}{lll}-1.40717600 & 3.34958000 & 1.33055400\end{array}$

$\begin{array}{llll}-1.00837500 & 4.71998000 & 1.83695400\end{array}$

$\begin{array}{lll}-1.29590400 & 5.48767500 & 1.11219900\end{array}$

$\begin{array}{lll}0.06566000 & 4.80265300 & 2.00538300\end{array}$

$\begin{array}{lll}-1.52224800 & 4.93335600 & 2.77517300\end{array}$

$\begin{array}{lll}-4.47960300 & 1.34472000 & 2.48142100\end{array}$

$\begin{array}{lll}-4.92871300 & 2.28057000 & 2.81606700\end{array}$

$\begin{array}{lll}-4.21556900 & 0.74919400 & 3.36227600\end{array}$

$\begin{array}{lll}-5.21497300 & 0.76819600 & 1.91777100\end{array}$

$0.61625400 \quad 3.09269600 \quad-0.10517900$

$\begin{array}{llll}0.78897500 & 2.39102500 & -0.93414800\end{array}$

$\begin{array}{llll}0.59685700 & 4.46997500 & -0.78256400\end{array}$

$\begin{array}{llll}-0.29859600 & 4.52255400 & -1.40759500\end{array}$

$\begin{array}{llll}0.54904200 & 5.27664200 & -0.04459300\end{array}$

$1.86141500 \quad 4.66482400 \quad-1.61965600$

$\begin{array}{llll}1.88531800 & 3.90782700 & -2.41148300\end{array}$

$\begin{array}{lll}1.83647800 & 5.64512600 & -2.10659700\end{array}$

$\begin{array}{llll}3.10039300 & 4.53846900 & -0.73309600\end{array}$

$\begin{array}{lll}3.10695100 & 5.35072800 & 0.00784000\end{array}$

$4.01490000 \quad 4.64336400-1.32626100$

$3.10896500 \quad 3.19009400-0.01487600$

$\begin{array}{llll}3.16476800 & 2.38024700 & -0.75541300\end{array}$

$\begin{array}{llll}3.99071000 & 3.08775300 & 0.62985500\end{array}$

$\begin{array}{lll}1.84065600 & 2.94336900 & 0.82344500\end{array}$

$\begin{array}{lll}1.77977300 & 3.69184200 & 1.62241500\end{array}$

$\begin{array}{lll}2.56607500 & 1.37914200 & 2.53560500\end{array}$

$\begin{array}{lll}2.96392200 & 0.09143500 & 2.96928900\end{array}$

$\begin{array}{llll}3.43570000 & 0.03837800 & 3.94127100\end{array}$

$3.07788700-1.04441100 \quad 2.15951600$

$3.98529200-2.15773700 \quad 2.61584300$

$\begin{array}{lll}4.87891300 & -2.19252000 & 1.98369400\end{array}$

$3.48945500-3.12855600 \quad 2.51819200$

$\begin{array}{lll}4.29386700 & -2.00510000 & 3.65047000\end{array}$

$\begin{array}{lll}2.94581800 & 2.53839200 & 3.43029000\end{array}$

$\begin{array}{lll}3.66349100 & 3.20137700 & 2.93770600\end{array}$

$3.39391900 \quad 2.17842700 \quad 4.35624200$

$2.06159200 \quad 3.13367100 \quad 3.68015100$

$2.89418400-2.17211100 \quad 0.04814500$

$2.22697600-3.40654200-0.00194900$

$2.67834000-4.35890700 \quad-0.91555500$ 

$-0.9446731 .8396621 .763231$ $\begin{array}{llll}1.335533 & 0.012117 & 2.315085\end{array}$ $1.810528-2.1227720 .465734$

$-3.515451-0.907980-0.088478$ $-3.195334-2.0777380 .636474$ $-3.599545-3.3059390 .113735$

$-3.374428-4.2193500 .658858$ $-4.294709-3.376419-1.090861$ $-4.603183-4.340920-1.483198$ $-4.589296-2.213856-1.790416$ $-5.124492-2.275961-2.734185$ $-4.201132-0.959079-1.309529$ $-2.504914-2.0174151 .994373$ $-2.010088-1.0458482 .097642$ $-1.439650-3.1056202 .175530$ $-0.789962-2.8504173 .018728$ $-0.804844-3.2257181 .291297$ $-1.894185-4.0806742 .385137$ $-3.527591-2.1133223 .136464$ $-3.014561-2.1284204 .104187$ $-4.115311-3.0337783 .047442$ $-4.217006-1.2647063 .136809$ $-4.5415170 .295041-2.093756$ $-4.2465731 .158876-1.487213$ $-3.7478230 .354946-3.404981$ $-4.0380611 .244667-3.974119$ $-3.961037-0.524869-4.024385$ $-2.6731150 .406692-3.212546$ $\begin{array}{lll}-6.048929 & 0.390239 & -2.362297\end{array}$ $-6.6363680 .295837-1.442658$ $-6.377815-0.397632-3.048784$ $-6.2869911 .351591-2.827756$ $-3.7774490 .9199611 .442840$ $-3.2451341 .7364642 .432228$ -3.945966 2.117068 3.164557 $-1.8855692 .1072852 .635293$ $-1.6089442 .8489903 .924539$ $-1.6628183 .9296883 .760465$ $-0.6221112 .6144074 .325980$

$-2.3596772 .5851034 .671209$ $-5.2615280 .6433401 .479816$ $-5.7192051 .0665642 .374764$ $-5.474301-0.4278631 .440206$ $\begin{array}{llll}-5.727228 & 1.095585 & 0.596407\end{array}$ $\begin{array}{llll}0.451689 & 2.296614 & 1.799195\end{array}$ $\begin{array}{llll}0.789059 & 2.191060 & 0.761341\end{array}$ $\begin{array}{llll}0.634373 & 3.798097 & 2.058277\end{array}$ $\begin{array}{lll}-0.106624 & 4.333602 & 1.453887\end{array}$ 0.4640694 .0555143 .108665 $2.055922 \quad 4.216884 \quad 1.682531$ $2.218713 \quad 4.037268 \quad 0.610835$ $2.1865555 .290203 \quad 1.851771$ 3.0647413 .4161542 .507013 2.9430433 .6645803 .571040 $\begin{array}{llll}4.089591 & 3.685859 & 2.233200\end{array}$ $2.862354 \quad 1.9145832 .304350$ 3.0543221 .6590581 .252703 $\begin{array}{llll}3.576708 & 1.334719 & 2.900819\end{array}$ $\begin{array}{llll}1.430226 & 1.443441 & 2.633163\end{array}$ $1.2234391 .608038 \quad 3.697864$ $1.714107-0.9046363 .193906$ $1.945853-2.2581842 .863844$ $2.177363-2.9199263 .688084$ $2.118589-2.7883641 .578797$ $2.736006-4.1569931 .458827$ $3.808536-4.0743141 .257720$ $2.289309-4.7125660 .630286$ $2.605784-4.7130402 .388601$ $1.947533-0.5168334 .637502$ $2.768088 \quad 0.200778 \quad 4.729893$ $2.193968-1.3940855 .235204$ $1.051214-0.0497835 .058461$ $2.318093-2.527228-0.815122$ $1.423204-2.984474-1.797627$ $1.936166-3.317875-3.052695$ $1.257934-3.680119-3.820129$ $3.288232-3.186163-3.333860$ $3.668822-3.447559-4.316263$ $4.151804-2.701215-2.358842$ $5.204506-2.570145-2.594022$ $3.688778-2.348757-1.091455$ $-0.061815-3.163886-1.533914$ $-0.297212-2.740689-0.554014$ $-0.926445-2.415765-2.553179$ $-0.651764-1.359322-2.632683$ $-1.976137-2.471297-2.246378$ $-0.845715-2.872434-3.546705$ $-0.431690-4.649990-1.473402$ $0.132664-5.175236-0.694401$ $-0.221369-5.140515-2.430341$ $-1.500517-4.760231-1.261540$ $4.659561-1.729118-0.094651$ $4.158232-1.6241960 .873922$ $5.050895-0.317905-0.552496$ $4.170668 \quad 0.304169-0.716389$ $5.604110-0.357514-1.497441$ $\begin{array}{llll}5.692533 & 0.162028 & 0.194879\end{array}$ $5.918180-2.5828170 .105407$ $6.538270-2.1595600 .901926$ $\begin{array}{lll}6.525866 & -2.603538 & -0.805085\end{array}$ $5.684230-3.6197500 .366362$ $0.651473-0.5960960 .554066$ $\begin{array}{llll}-1.231147 & 0.764643 & 0.114416\end{array}$ $-0.0619231 .869599-1.496948$ $1.3268651 .671632-1.449928$ $-0.3793853 .199137-1.868221$ $\begin{array}{llll}0.527912 & 4.056478 & -2.443668\end{array}$ $2.2800712 .484402-2.028872$ $\begin{array}{llll}1.880610 & 3.703304 & -2.554964\end{array}$ $-0.7287400 .734625-2.485643$ $-1.6276023 .670836-1.597666$ $\begin{array}{lll}1.855668 & 0.541992 & -0.718294\end{array}$ $\begin{array}{llll}3.579415 & 2.144201 & -2.038289\end{array}$ $0.1576055 .283443-2.836839$ $2.743274 \quad 4.484249-3.231206$

TS2b

$\begin{array}{llll}\mathrm{N} & 2.97041200 & -0.29104500 & 0.53917000\end{array}$ N $\quad 0.94452500 \quad-2.05557700 \quad 1.64169300$ N $\quad \begin{array}{llll}\mathrm{N} & -1.47498400 & -0.45677100 & 2.28268800\end{array}$ $\begin{array}{llll}N & -2.06679300 & 1.79579800 & 0.61818100\end{array}$ 

$\begin{array}{llll}0.59736000 & 3.05336200 & -3.23998000\end{array}$ $0.02672900 \quad 4.65292800-1.06366400$ $\begin{array}{llll}-0.58811700 & 5.08606700 & -0.26655800\end{array}$ $-0.19494000 \quad 5.19291100-1.99090900$ $\begin{array}{llll}1.08097800 & 4.81815500 & -0.81743400\end{array}$ $\begin{array}{llll}-4.86729500 & 1.25307600 & -0.04522600\end{array}$ $\begin{array}{lll}-4.37618900 & 1.09463400 & 0.92125100\end{array}$ $\begin{array}{lll}-5.14610200 & -0.13358900 & -0.63977900\end{array}$ $\begin{array}{lll}-4.22050400 & -0.67341600 & -0.84113100\end{array}$ $\begin{array}{lll}-5.68675600 & -0.04507500 & -1.58878400\end{array}$ $\begin{array}{lll}-5.76233600 & -0.72600500 & 0.04584700\end{array}$ $\begin{array}{lll}-6.18827100 & 1.99222300 & 0.20289400\end{array}$ $\begin{array}{lll}-6.79015100 & 1.45097900 & 0.93974700\end{array}$ $\begin{array}{lll}-6.77908600 & 2.05763600 & -0.71658300\end{array}$ $\begin{array}{lll}-6.03562800 & 3.01295700 & 0.56742500\end{array}$ $\begin{array}{lll}-0.79895400 & 0.35398800 & 0.59964400\end{array}$ $\begin{array}{lll}1.18622300 & -0.82611600 & 0.09409100\end{array}$ $\begin{array}{llll}0.13145300 & -1.86971400 & -1.63300700\end{array}$ $\begin{array}{llll}-1.27230900 & -1.78288300 & -1.59816400\end{array}$ $0.55383900-3.12737900-2.13406200$ $-0.28900200-3.98156100-2.80060700$ $\begin{array}{lll}-2.14206500 & -2.61452400 & -2.26814500\end{array}$ $\begin{array}{lll}-1.66622400 & -3.74121700 & -2.92015300\end{array}$ $0.73165300 \quad-0.60951300-2.50592200$ $\begin{array}{llll}0.20499600 & -5.12915000 & -3.29974500\end{array}$ $\begin{array}{llll}1.84408800 & -3.50865400 & -1.92211100\end{array}$ $-2.30862500-4.37975800-3.50945500$ $\begin{array}{lll}-1.89250300 & -0.75406600 & -0.79340900\end{array}$ $\begin{array}{lll}-3.46634500 & -2.36497200 & -2.23138300\end{array}$

TS2

$\begin{array}{lll}-3.027253 & 0.391473 & 0.364116\end{array}$ $\begin{array}{lll}-0.9985242 .369647 & 0.989906\end{array}$ $\begin{array}{lll}1.437976 & 0.977716 & 1.953712\end{array}$ $2.017033-1.6009580 .856530$ $-3.393625-0.9912800 .257192$ $-2.958282-1.8505861 .291025$ $-3.281997-3.2041721 .199729$ $-2.967872-3.8836971 .988206$ $-4.008593-3.6947410 .117656$ $-4.252615-4.7515690 .062867$ $-4.415929-2.832960-0.891805$ $-4.974835-3.223051-1.738293$ $-4.112246-1.468390-0.847315$ $-2.229010-1.3214892 .521059$ $-1.804328-0.3398512 .284673$ $-1.080909-2.2305742 .976678$ $-0.430215-1.6852903 .667592$ $-0.462012-2.5778262 .142634$ $-1.455702-3.1187763 .498180$ $-3.201736-1.1118643 .691422$ $-2.654775-0.7935054 .585551$ $-3.724725-2.0457163 .926164$ $-3.949446-0.3464123 .467294$ $-4.575191-0.548681-1.962543$ $-4.3297490 .479081-1.671865$ $-3.831616-0.852056-3.269354$ $-4.209360-0.206285-4.069474$ $-3.995282-1.893667-3.571993$ $-2.758432-0.673027-3.163271$ $-6.093374-0.639763-2.163246$ $-6.641240-0.476552-1.228912$ $-6.381970-1.623332-2.549998$ $-6.4210410 .108401-2.891651$ $-3.7481731 .2010081 .160658$ $-3.2504702 .3229151 .810184$ -3.9517332.8655572.431675 $-1.9203472 .8320001 .797056$ $\begin{array}{lll}-1.6556153 .960526 & 2.769703\end{array}$

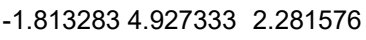
$-0.6351573 .9364313 .154423$ $-2.3465573 .8928713 .611670$ $-5.2013820 .8491301 .375511$ $-5.6570891 .4966112 .125710$ $-5.325346-0.1934911 .678473$ $\begin{array}{llll}-5.738061 & 0.973323 & 0.427652\end{array}$ $\begin{array}{llll}0.354167 & 2.908816 & 0.791454\end{array}$ $0.6602752 .500003-0.178211$ $0.419204 \quad 4.422364 \quad 0.547878$ $-0.3839594 .681556-0.151175$ 0.2641834 .9929951 .469499 
$\begin{array}{llll}1.786622 & 4.791747 & -0.028394\end{array}$

TS3a

$\mathrm{Ga}$

Ga

$\mathrm{N}$

$\mathrm{N}$

$\mathrm{N}$

N

C

C

$\mathrm{H}$

$\mathrm{C}$

C $-1.562959-1.4060512 .026116$ $\begin{array}{llll}1.922113 & 4.289233 & -0.996067\end{array}$ $\begin{array}{llll}1.834409 & 5.869320 & -0.214931\end{array}$ $2.888930 \quad 4.366869 \quad 0.943076$ $\begin{array}{llll}2.786694 & 4.933803 & 1.879638\end{array}$ 3.8767624 .6030990 .534909 $2.805603 \quad 2.869258 \quad 1.238759$ $\begin{array}{llll}977405 & 2.304191 & 0.312054\end{array}$ 3.5876252 .5606331 .942854 $\begin{array}{llll}1.430961 & 2.437333 & 1.790468\end{array}$ $\begin{array}{lll}1.254511 & 2.924423 & 2.757519\end{array}$ $\begin{array}{llll}1.915477 & 0.419877 & 3.055657\end{array}$ $2.236291-0.9518823 .162769$ $2.543850-1.2979684 .140811$ $2.407587-1.8543162 .105597$ $3.119398-3.1501632 .394736$ $4.173924-3.0772502 .111616$ $2.681890-3.9689951 .817674$ $3.067238-3.3807483 .459968$ $2.171630 \quad 1.266793 \quad 4.282975$ 2.9391632 .0226834 .092041 $2.5047650 .646095 \quad 5.114486$ $1.258926 \quad 1.7912994 .584030$ $2.515742-2.364735-0.252053$ $1.632879-3.177018-0.984244$ $2.134350-3.866625-2.089979$ $1.465113-4.503576-2.661627$ $3.462843-3.744943-2.470380$ $3.834596-4.287173-3.334197$ $4.312579-2.912227-1.751961$ $5.344386-2.795745-2.072278$ $3.859279-2.196526-0.644234$ $0.175109-3.358988-0.598774$ $0.059682-2.6708000 .217062$ $-0.775259-3.016576-1.750367$ $-0.588939-2.019153-2.161064$ $-1.808034-3.046812-1.387671$ $-0.685632-3.748329-2.562131$ $-0.079814-4.774597-0.069466$ $0.547806-4.9996510 .800364$ $0.134276-5.522084-0.841489$ $-1.129752-4.8793670 .223917$ $4.805088-1.2218270 .044941$ $4.322870-0.8370990 .950387$ $5.071834-0.018142-0.868345$ $\begin{array}{llll}4.141972 & 0.454627 & -1.185344\end{array}$ $5.605441-0.330997-1.772891$ $\begin{array}{llll}5.690649 & 0.723716 & -0.351052\end{array}$ $6.131732-1.8778670 .448065$ $6.739973-1.1742541 .025276$ $6.711997-2.162351-0.435808$ $5.987694-2.7808111 .049739$ $0.738566-0.2064960 .519262$ $-1.2554550 .815931-0.234185$ $-0.2196761 .420153-2.169794$ $\begin{array}{llll}1.180462 & 1.363807 & -2.129415\end{array}$ $-0.6506412 .507241-2.980557$ $0.1547723 .201187-3.843863$ $2.0372072 .013247-2.997111$ $\begin{array}{llll}1.539811 & 2.937323 & -3.897548\end{array}$ $-0.822501-0.012652-2.712011$ -1.962029 2.870318 -2.860209 $2.1943803 .415465-4.612420$ $\begin{array}{llll}1.817667 & 0.568884 & -1.100349\end{array}$ $3.3656381 .787896-2.908000$ $-0.2821553 .983163-4.457514$

$\begin{array}{lll}1.171430 & 0.116208 & 0.142401\end{array}$ $\begin{array}{llll}-1.227512 & -0.444332 & 0.264751\end{array}$ $\begin{array}{llll}1.582897 & 1.840375 & 0.894841\end{array}$ $1.433000-0.8287091 .929618$ $-3.100539-0.395318-0.160631$ 0.82418329978310 .537069 $-0.2346933 .3634821 .393114$ $-0.8990424 .5661461 .148450$ $\begin{array}{lll}-1.6956544 .884405 & 1.817177\end{array}$ $\begin{array}{lll}-0.553475 & 5.362830 & 0.062147\end{array}$ $\begin{array}{lll}-1.074327 & 6.299780 & -0.112966\end{array}$ $0.4345294 .940132-0.818603$
$0.6727045 .545992-1.689051$ $1.1391153 .753758-0.601572$

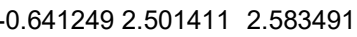
$-0.1051141 .5463542 .534583$ $-2.1411242 .1899872 .557544$ $-2.3900091 .4512273 .326364$ $-2.4539741 .7854251 .586201$ $-2.7442783 .0857832 .743371$ $\begin{array}{lll}-0.260875 & 3.157460 & 3.917164\end{array}$ $-0.5963372 .5396114 .757749$ $-0.7282354 .1439124 .014470$ $0.822675 \quad 3.282222 \quad 4.003792$ $2.2114653 .308017 \quad-1.577488$ $2.7285372 .452866-1.132506$ $\begin{array}{llll}1.573051 & 2.852617 & -2.897748\end{array}$ $2.3513882 .505390-3.587396$ $1.053673 \quad 3.692828-3.376171$ $\begin{array}{llll}0.853581 & 2.044689 & -2.727925\end{array}$ $3.2571844 .401458-1.824076$ $\begin{array}{llll}3.697564 & 4.763568 & -0.889004\end{array}$ $2.818960 \quad 5.261582 \quad-2.342441$ $\begin{array}{llll}4.063667 & 4.013785 & -2.454571\end{array}$ $2.4363331 .972654 \quad 1.926834$ $\begin{array}{llll}2.740204 & 0.960918 & 2.826331\end{array}$ $\begin{array}{llll}3.425785 & 1.212787 & 3.626311\end{array}$ $2.118941-0.3189052 .919530$ $2.303180-1.0153074 .253466$ $3.180719-1.6692814 .225821$ $1.437081-1.6201764 .524520$ $2.463658-0.2732835 .037291$ $3.055050 \quad 3.3315582 .160749$ $3.569797 \quad 3.649737 \quad 1.247355$ $3.771347 \quad 3.2985332 .982944$ $2.296234 \quad 4.0876952 .380795$ $0.840129-2.1657151 .879912$ $0.649023-2.3433820 .809190$ $1.816345-3.2891682 .262038$ $2.768571-3.0803221 .760606$ $2.009406-3.3107343 .338484$ $1.259159-4.6516171 .856812$ $1.111213-4.6812480 .768384$ $1.973806-5.4417402 .108063$ $-0.074735-4.8788972 .565238$ $0.090626-4.9070273 .651367$ $-0.507940-5.8448132 .285431$ $-1.055905-3.7613382 .219381$ $-1.258360-3.7797631 .139103$ $-2.021224-3.9228282 .710675$ $-0.547937-2.3385142 .546067$ $-0.434438-2.2234453 .632498$ $-2.694625-1.2430552 .693824$ $-3.872766-0.7451242 .099730$ $-4.741216-0.6742132 .741308$ $-4.083237-0.4912920 .742893$ $\begin{array}{lll}-5.504761 & -0.333551 & 0.267667\end{array}$ $-5.749233-1.126499-0.447203$ $\begin{array}{llll}-5.634789 & 0.617197 & -0.257565\end{array}$ $-6.201090-0.3832271 .104944$ $-2.784395-1.6117384 .160487$ $-2.660543-2.6850944 .328270$ $-3.748066-1.3118484 .572669$ $-1.994164-1.0984914 .718917$ $-3.423112-0.340761-1.564485$ $\begin{array}{llll}-3.642837 & 0.889838 & -2.195912\end{array}$ $-3.9085430 .880855-3.566873$ $-4.0707411 .824576-4.080093$ $-3.954292-0.306320-4.283618$ $-4.159110-0.290245-5.349683$ $-3.728253-1.516840-3.638306$ $-3.755617-2.440509-4.208270$ $-3.455030-1.555724-2.271828$ $-3.5870152 .206732-1.444919$ $-3.5323031 .986759-0.372309$ $-2.3249162 .992596-1.818232$ $-1.4231402 .412062-1.599327$ $-2.3012923 .937981-1.269913$ $-2.3260833 .220071-2.891713$ $-4.8419093 .055763-1.683829$ $-5.7657062 .506337-1.471295$ $-4.8930693 .399615-2.722280$ $-4.8172793 .945267-1.046431$ $-3.108569-2.866732-1.582050$ 
$-3.346709-2.766741-0.515085$ $-1.601703-3.119281-1.703220$ $-1.031227-2.311199-1.232605$ $-1.289180-3.140289-2.751987$ $-1.310349-4.064142-1.229721$ $-3.901093-4.061711-2.115808$ $-3.711716-4.942878-1.494857$ $-3.607609-4.317740-3.138906$ $-4.977153-3.862111-2.111294$ $4.938113-0.792830-1.697575$ $4.683926-1.968920-2.389534$ $3.418932-2.539508-2.338375$ $2.431653-1.905548-1.597977$ $2.631494-0.723443-0.908141$ $3.912807-0.204659-0.968101$ $1.228890-2.513700-1.549817$ $-0.3052950 .671186-1.683853$ $\begin{array}{llll}4.230646 & 0.913673 & -0.290076\end{array}$ $3.180477-3.681154-2.985058$ $6.159230-0.258974-1.734254$ $5.652811-2.555729-3.087765$

\section{TS3b}

Ga

$\mathrm{Ga}$

N

$\mathrm{N}$

$\mathrm{N}$

$\mathrm{N}$

C
$1.270275-0.129517-0.037992$

$\begin{array}{llll}-1.167891 & -0.333076 & 0.275071\end{array}$

$2.003760 \quad 1.427336 \quad 0.827476$

$1.530452-1.2850281 .621188$

$-1.500632-1.4165591 .967152$

$\begin{array}{lll}-3.039320 & 0.042119 & 0.050622\end{array}$

$\begin{array}{lll}1.403166 & 2.713481 & 0.660723\end{array}$

$\begin{array}{llll}0.486691 & 3.129086 & 1.648396\end{array}$

$-0.0080924 .4327441 .586284$

$-0.6893154 .7852232 .357404$

$\begin{array}{lll}0.363955 & 5.287684 & 0.554524\end{array}$

$-0.0229286 .3021860 .522433$

$1.203804 \quad 4.830345-0.453536$

$1.4596875 .489683-1.278921$

$1.7360853 .539315-0.423108$

$\begin{array}{llll}0.064588 & 2.211374 & 2.791062\end{array}$

$0.431863 \quad 1.197702 \quad 2.591583$

$-1.9327851 .8869181 .953801$

-1.888673 3.085173 3.249213

0.6777432 .6521034 .126873

$\begin{array}{llll}0.333451 & 2.002471 & 4.939527\end{array}$

$0.386705 \quad 3.6806314 .368871$

$\begin{array}{lll}1.770352 & 2.603381 & 4.098463\end{array}$

$2.6471133 .058622-1.536978$

$\begin{array}{llll}3.070073 & 2.097730 & -1.229407\end{array}$

$\begin{array}{llll}1.841068 & 2.840356 & -2.825714\end{array}$

$2.4994632 .464219-3.617529$

$\begin{array}{llll}1.413526 & 3.790947 & -3.169746\end{array}$

$1.0263322 .126834-2.663743$

$3.8164064 .019596-1.780501$

$4.380577 \quad 4.217103-0.862789$

$\begin{array}{llll}3.466611 & 4.981512 & -2.171649\end{array}$

$\begin{array}{llll}4.502907 & 3.594444 & -2.519529\end{array}$

$2.9567401 .321962 \quad 1.771440$

$3.177643 \quad 0.1859952 .536794$

3.9628650 .2470093 .280549

$2.372028-0.9896502 .577557$

$2558342-1.8407053 .818318$

$3.313735-2.6134413 .642985$

$1.633305-2.3323414 .121923$

.903462 -1.216684 4.644315

$3.7996342 .542252 \quad 2.062129$

$4.2810822 .869774 \quad 1.133914$

4.5672442 .3195322 .804698

3.1906713 .3773112 .419833

$0.731431-2.5062081 .511065$

$0.424766-25411530.453298$

$1.546432-3.7961221 .692522$

$2.474642-3.6803711 .120927$

$1.821993-3.9583662 .738645$

$0.749402-5.0105331 .222727$

$1.348823-5.9201711 .330044$

$-0.539514-5.1094772 .036047$

$-0.290317-5.2758183 .093456$

$-1.142035-5.9652271 .714003$

$-1.357152-3.8280121 .892104$
$-1.4604612 .1341492 .913222$

$-1.7455331 .3635093 .636980$

$0.508469-4.9039270 .156049$
$-1.649889-3.7027340 .839982$

$-2.291140-3.8945432 .460109$

$-0.604721-2.5390742 .294636$

$-0.382422-2.5581123 .370189$

$-2.530623-1.1596832 .758034$

$-3.661755-0.4312472 .334799$

$-4.449854-0.3010123 .064739$

$-3.943884-0.0033041 .036217$

$\begin{array}{lll}-5.357612 & 0.416325 & 0.724728\end{array}$

$-5.786964-0.249706-0.031328$

$\begin{array}{llll}-5.378355 & 1.426341 & 0.305177\end{array}$

$\begin{array}{lll}-5.977884 & 0.383198 & 1.620591\end{array}$

$-2.552256-1.6660154 .185850$

$-2.587136-2.7575504 .239987$

$-3.417054-1.2709784 .719332$

$-1.645612-1.3401164 .706905$

$-3.4707220 .291204-1.302014$

$-3.5460421 .599558-1.795733$

$-3.9355191 .773183-3.125699$

$-3.9903462 .778841-3.533221$

$-4.2372930 .687588-3.935278$

$-4.5349710 .845034-4.967429$

$-4.147645-0.603750-3.427807$

$-4.373058-1.448681-4.071314$

$-3.759498-0.825077-2.107395$

$-3.2060722 .808548-0.944859$

$\begin{array}{lll}-3.0733602 .473083 & 0.090437\end{array}$

$-1.8822863 .434789-1.397963$

$-1.0658102 .708782-1.333170$

$-1.6545654 .306909-0.779557$

$-1.9588043 .766111-2.441242$

$-4.3291693 .853320-0.958318$

$-5.3009533 .428142-0.684031$

$-4.4342154 .305269-1.950313$

$-4.0966614 .659395-0.255248$

$-3.560601-2.237699-1.578659$

$-3.682175-2.216455-0.487830$

$-2.128747-2.693470-1.881257$

$-1.398045-2.031615-1.403952$

$-1.920353-2.650478-2.954915$

$-1.945834-3.714908-1.527630$

$-4.574995-3.241378-2.130548$

$-4.469642-4.200883-1.614630$

$-4.419182-3.429064-3.197760$

$-5.601872-2.888108-1.994403$

$4.660958-1.388265-2.333943$

$4.190913-2.445112-3.096433$

$2.864041-2.817073-2.948042$

$2.043312-2.131751-2.063281$

$2.481660-1.061266-1.304810$

$3.815495-0.725150-1.452679$

$4.843474-2.970073-3.783134$

$0.769066-2.562965-1.938158$

$-0.2502820 .833990-1.645313$

$\begin{array}{llll}4.355704 & 0.267443 & -0.717873\end{array}$

$2.372848-3.845306-3.649359$

$5.939776-1.009597-2.434786$

TS3c

$\mathrm{Ga}$

$\mathrm{Ga}$

$\mathrm{N}$

N

$\mathrm{N}$

$\mathrm{N}$

C

C

H

C
$1.342884-0.297367-0.200037$ $\begin{array}{llll}-1.067769 & -0.241071 & 0.319271\end{array}$ $2.307608 \quad 1.1419020 .646331$ $1.604502-1.5284811 .405493$ $-1.381145-1.3410182 .006029$ $\begin{array}{llll}-2.899458 & 0.342873 & 0.267710\end{array}$ $1.843646 \quad 2.490475 \quad 0.557172$ $\begin{array}{lll}1.066611 & 2.976269 & 1.628812\end{array}$ $0.719853 \quad 4.328206 \quad 1.636444$ $\begin{array}{llll}0.150440 & 4.731125 & 2.470995\end{array}$ $\begin{array}{llll}1.096875 & 5.166425 & 0.592743\end{array}$ $\begin{array}{llll}1.788236 & 4.647525 & -0.495076\end{array}$ $\begin{array}{llll}2.044086 & 5.297497 & -1.327987\end{array}$ $2.171022 \quad 3.304927-0.536911$ 0.6366142 .0760922 .782390 0.8713871 .0349632 .531002 $-0.8734342 .1630843 .025195$ $-1.1866191 .4056213 .751223$ $\begin{array}{lll}-1.442082 & 2.0004562 .100629\end{array}$ $\begin{array}{ll}-1.165209 & 3.144270 \\ 3.416226\end{array}$ $\begin{array}{lll}1.397515 & 2.405351 & 4.073470\end{array}$ $1.0461621 .773642 \quad 4.897171$ 0.8264626 .2182520 .614785 
$3.676179-1.114529-1.877155$ $0.417520-2.583990-2.125728$ $-0.1848860 .904812-1.629933$ $4.365275-0.210047-1.142088$ $1.700782-3.948627-4.021301$ $5.433235-1.597237-3.008299$ $4.207301-3.348855-4.366324$

TS4

$\mathrm{Ga}$

$\mathrm{N}$

$\mathrm{N}$

C

$\mathrm{H}$

$\mathrm{H}$

H

$\mathrm{H}$

$\mathrm{C}$

$2.506829-0.5716671 .031997$

$\begin{array}{lll}-1.269799 & 1.910528 & 0.812122\end{array}$ $-0.383563-0.4122852 .234013$ $1.041355-1.6739700 .109744$

$3.289034-0.150644-0.782284$ $-1.9992272 .711004-0.128186$ $-1.6561132 .681558-1.483844$ $-2.4015713 .467194-2.370978$ $-2.1525933 .456425-3.428721$ $-3.4567614 .243584-1.924710$ $-4.0229454 .848713-2.625837$ $-3.8084894 .230038-0.576161$ $\begin{array}{lll}-4.654520 & 4.822465 & -0.245251\end{array}$ $\begin{array}{lll}-3.1040513 .458897 & 0.342832\end{array}$ $-0.5748021 .768588-2.038792$ $-0.0264411 .296810-1.211460$ $0.4672852 .527260 \quad-2.865204$ $0.918847 \quad 3.338535-2.285192$ $0.0283432 .961627 \quad-3.769832$ $1.2627521 .840817-3.176272$ $-1.2307250 .659471-2.873033$ $\begin{array}{lll}-1.908067 & 0.052949 & -2.261613\end{array}$ $\begin{array}{llll}-0.471661 & 0.002371 & -3.305513\end{array}$ $-1.8249021 .088269-3.687313$ $\begin{array}{lll}-3.536537 & 3.365750 & 1.803679\end{array}$ $-2.6500823 .5009062 .436723$ $\begin{array}{lll}-4.5520674 .427574 & 2.220980\end{array}$ $-4.1943195 .4414832 .014537$ $-4.7466764 .3467193 .294415$ $-5.5073644 .2883051 .704091$ $-4.0805821 .9617122 .094114$ $\begin{array}{lll}-3.3611181 .228360 & 1.742673\end{array}$ $-5.0145511 .7826691 .553101$ $-4.2498301 .8093593 .164070$ $-0.4666832 .5242541 .687157$ $0.109145 \quad 1.8848192 .783520$ $0.6647722 .508542 \quad 3.471884$ $0.1237820 .505503 \quad 3.054515$ $\begin{array}{llll}0.881471 & 0.091398 & 4.292985\end{array}$ $1.904875-0.1867834 .008624$ $0.415351-0.7566584 .798267$ $\begin{array}{llll}0.936586 & 0.927611 & 4.991228\end{array}$ $-0.1493863 .9871581 .481998$ $\begin{array}{llll}0.366500 & 4.106448 & 0.521275\end{array}$ $\begin{array}{llll}0.495219 & 4.357203 & 2.279493\end{array}$ $-1.0487654 .6048651 .434767$ $0.854597-3.8704611 .277837$ $1.820179-3.7285331 .786868$ $-0.078926-4.7419122 .124678$ $-0.990135-4.9443131 .550096$ $0.395068-5.7079582 .327470$ $-0.455116-4.0525613 .440674$ $-1.187789-4.6555753 .986202$ $0.437254-3.9810844 .078611$ $-1.007629-2.6435893 .202209$ $-1.216238-2.1384864 .151943$ $-1.954603-2.6860322 .651956$ $0.006250-1.8178852 .402526$ $0.965878-1.8483122 .930936$ $0.227723-2.4915491 .025859$ $-0.762903-2.6512920 .593155$ $0.853918-1.816069-1.202694$ $1.639118-1.179237-2.181257$ $1.351001-1.351672-3.210011$ $2.791163-0.412417-1.986023$ $3.5034840 .111552-3.212356$ $4.466606-0.392107-3.348578$ $\begin{array}{llll}3.719044 & 1.178767 & -3.098753\end{array}$ $2.899752-0.040605-4.108525$ 
$-0.284891-2.660898-1.741170$

$-1.239408-2.142436-1.583795$

$-0.362117-3.638406-1.258737$

$-0.168894-2.814910-2.814473$

$\begin{array}{llll}4.579311 & 0.460197 & -0.678934\end{array}$

$\begin{array}{llll}4.690789 & 1.810493 & -0.314186\end{array}$

$\begin{array}{llll}5.971591 & 2.358529 & -0.181938\end{array}$

$\begin{array}{llll}6.075501 & 3.404044 & 0.096627\end{array}$

$\begin{array}{llll}7.106409 & 1.593621 & -0.397408\end{array}$

$\begin{array}{llll}8.091594 & 2.038242 & -0.296059\end{array}$

$\begin{array}{llll}6.980168 & 0.247718 & -0.730993\end{array}$

$7.874613-0.348652-0.879798$

$\begin{array}{llll}5.726726 & -0.344166 & -0.863654\end{array}$

$3.4807232 .692616-0.047961$

$2.5701322 .112904-0.247250$

$\begin{array}{llll}3.464153 & 3.910507 & -0.980114\end{array}$

$3.4732283 .609475-2.032773$

$\begin{array}{llll}4.333221 & 4.554233 & -0.807254\end{array}$

$2.567187 \quad 4.515314-0.805218$

$\begin{array}{llll}3.450107 & 3.138143 & 1.420039\end{array}$

2.5931653 .7946941 .599925

$4.3581193 .696078 \quad 1.674526$

$\begin{array}{llll}3.375254 & 2.277704 & 2.093011\end{array}$

$5.588084-1.840773-1.112218$

$4.698441-2.010557-1.728931$

$\begin{array}{llll}5.345275 & -2.549449 & 0.227597\end{array}$

$4.482151-2.1016720 .728032$

$6.209602-2.4267550 .888895$

$5.156052-3.6183840 .085868$

$6.779210-2.459663-1.844210$

$7.012680-1.918165-2.766787$

$6.555477-3.498724-2.103516$

$7.677369-2.468412-1.217901$

$1.075216-4.3532650 .319288$

$-1.585727-0.0298410 .729899$

$-3.369547-1.3638220 .109594$

$-3.360224-2.748490-0.196462$

$-4.069835-0.586571-0.847363$

$-3.692216-1.1167001 .500502$

$-3.783344-3.255880-1.409829$

$-2.743523-3.5977040 .661826$

$-4.467514-1.076887-2.071820$

$-3.9862100 .754438-0.688408$

$-4.372934-2.435026-2.362875$

$-3.659370-4.564826-1.647271$

$-4.955919-0.239875-2.990355$

$-4.843387-2.933421-3.513808$

TS4b

$\mathrm{Ga}$

N

N

$\mathrm{N}$

N

C

C

$\mathrm{H}$

C

C

$\mathrm{H}$

C

C

C

$2.4253190 .597587-0.989180$ $-1.440535-1.742169-0.841364$ $-0.5083750 .674485-2.094812$

$\begin{array}{llll}1.051027 & 1.684131 & 0.075001\end{array}$ $\begin{array}{llll}3.269951 & 0.021525 & 0.752357\end{array}$ $-2.139280-2.6028120 .070832$ $-1.728581-2.6842511 .405216$ $-2.438774-3.5325862 .262960$ $-2.138149-3.6082893 .304435$ $-3.523927-4.2621421 .809847$ $-4.061916-4.9170872 .488057$ $-3.942308-4.1369830 .486465$ $-4.811393-4.6931380 .152624$ $-3.275027-3.300342-0.402436$ $-0.613184-1.8253421 .978533$ $-0.089768-1.3032311 .165144$ $0.449766-2.6538992 .705512$ $0.865810-3.4270282 .051466$ $0.040495-3.1465713 .593969$ $1.266667-2.0010983 .032378$ $-1.219918-0.7725942 .917432$ $-1.925519-0.1220432 .388299$ $-0.434455-0.1518413 .356407$ $-1.770780-1.2591003 .729782$ $-3.780006-3.084872-1.826276$ $-2.931193-3.186402-2.514954$ $-4.840034-4.090905-2.269863$ $-4.495747-5.124900-2.164670$ $-5.089351-3.920109-3.321214$ $-5.762956-3.975283-1.691936$ $-4.304370-1.652057-1.976446$ $-3.548841-0.962754-1.614794$ $-5.201956-1.497101-1.370351$
$-4.530639-1.415762-3.020389$ $-0.692267-2.299248-1.800405$ $-0.140998-1.584963-2.862503$ $0.362991-2.164632-3.624947$ $-0.075860-0.188645-3.012062$ $0.6565770 .302676-4.236640$ $\begin{array}{llll}1.701325 & 0.506982 & -3.967315\end{array}$ $0.2145361 .213282-4.645374$ $0.647130-0.468366-5.008071$ $-0.405053-3.780783-1.729930$ $0.143992-3.989612-0.803502$ $0.200920-4.094997-2.579855$ $-1.315712-4.382304-1.698041$ $\begin{array}{llll}0.927050 & 3.977410 & -0.896414\end{array}$ $\begin{array}{llll}1.865365 & 3.837751 & -1.454967\end{array}$ $0.0059524 .960398-1.626782$ $-0.8742115 .150372-1.001466$ $0.5173335 .918889-1.764257$ $-0.4497954 .405160-2.980397$ $-1.1703945 .086079-3.444192$ $0.4145164 .345719-3.657170$ $-1.0649893 .010021-2.833695$ $-1.3439952 .596969-3.809510$ $-1.9801323 .054016-2.232774$ $-0.0602092 .072270-2.154645$ $0.8779442 .105846-2.719611$ $0.2442842 .610930-0.736535$ $-0.7198232 .774809-0.251856$ $\begin{array}{llll}0.915554 & 1.729436 & 1.400936\end{array}$ $\begin{array}{lll}1.714665 & 0.992483 & 2.294671\end{array}$ $\begin{array}{llll}1.472837 & 1.092455 & 3.344771\end{array}$ $2.8325150 .209853 \quad 1.993137$ $3.580203-0.4219953 .145443$ $\begin{array}{llll}4.562130 & 0.045686 & 3.275789\end{array}$ $3.760093-1.4830212 .945076$ $3.019173-0.3211924 .075965$ $-0.1648762 .5772612 .045552$ $\begin{array}{lll}-1.1539932 .137270 & 1.861131\end{array}$ $-0.1888773 .5994801 .660063$ $-0.0176562 .6198783 .125185$ $4.534889-0.6159990 .548045$ $4.588454-1.9383680 .081851$ $5.844755-2.511883-0.144214$ $5.904043-3.536629-0.501826$ $7.011095-1.7977440 .077351$ $7.976775-2.261899-0.097608$ $6.941384-0.4766190 .511532$ $\begin{array}{llll}7.859681 & 0.081424 & 0.663781\end{array}$ $\begin{array}{llll}5.713783 & 0.139707 & 0.739845\end{array}$ $3.340588-2.761866-0.197174$ $2.458496-2.1721860 .083979$ $3.321345-4.0461480 .641134$ $3.383704-3.8263021 .711933$ $4.160367-4.7011700 .383157$ $2.397910-4.6080320 .460772$ $3.235323-3.093396-1.691746$ $2.351599-3.710269-1.882568$ $4.114036-3.655833-2.026488$ $3.159958-2.182465-2.294588$ $\begin{array}{llll}5.632646 & 1.617854 & 1.099537\end{array}$ $\begin{array}{llll}4.774112 & 1.768375 & 1.763493\end{array}$ $\begin{array}{llll}5.358659 & 2.427847 & -0.175318\end{array}$ $\begin{array}{llll}4.461956 & 2.042597 & -0.668667\end{array}$ $\begin{array}{llll}6.191066 & 2.328149 & -0.880015\end{array}$ $\begin{array}{llll}5.209306 & 3.488842 & 0.049513\end{array}$ 6.8710812 .1481531 .822450 $\begin{array}{llll}7.123927 & 1.535247 & 2.693828\end{array}$ $\begin{array}{llll}6.690900 & 3.171857 & 2.163767\end{array}$ $\begin{array}{llll}7.743339 & 2.176140 & 1.161072\end{array}$ $\begin{array}{llll}1.206134 & 4.364721 & 0.089787\end{array}$ $-1.6581970 .185503-0.601973$ $\begin{array}{llll}-3.314380 & 1.496263 & 0.248771\end{array}$ $\begin{array}{lll}-3.344729 & 2.862067 & 0.624563\end{array}$ $-4.1095040 .6727951 .083050$ $-3.5418391 .313872-1.210028$ $-4.0197583 .3157151 .740366$ $-2.6040223 .756856-0.084441$ $-4.7714651 .1333432 .197048$ $-4.015434-0.6649660 .871220$ $-4.7764432 .4794752 .550019$ $\begin{array}{ll}-3.9457974 .6253462 .021123 \\ -5.4201960 .245380 & 2.963632\end{array}$ $\begin{array}{lll}-5.420196 & 0.245380 & 2.963632\end{array}$ 
$-6.827245-0.854896-0.371102$ $-7.782952-0.373327-0.551680$ $-5.646860-0.177640-0.666204$ $-3.072171-2.8337740 .449859$

$-2.234997-2.2106490 .109755$

$-2.978011-4.174099-0.289831$ $-3.067714-4.040962-1.372930$

$-3.768537-4.8609140 .031029$ $-2.017257-4.659110-0.083560$ $-2.926808-3.0425171 .963028$ $-1.999370-3.5794882 .184908$ $-3.759923-3.6374872 .353702$ $-2.908527-2.0852692 .494274$ $-5.6699881 .269281-1.142250$ $-4.8318021 .422491-1.831213$ $\begin{array}{lll}-5.435291 & 2.194515 & 0.059838\end{array}$ $\begin{array}{lll}-4.509628 & 1.908498 & 0.567029\end{array}$ $\begin{array}{lll}-6.2512342 .097602 & 0.783944\end{array}$ $-5.3590413 .241664 \quad-0.250468$ $-6.9500891 .657269-1.882736$ $\begin{array}{lll}-7.172160 & 0.961523 & -2.698537\end{array}$ $-6.8433142 .660213-2.306681$ $-7.8139701 .680398-1.210235$ $-1.3480494 .374909-0.362233$ $\begin{array}{llll}1.730766 & 0.406577 & 0.491385\end{array}$ $\begin{array}{llll}3.334546 & 1.781809 & -0.427167\end{array}$ $3.1996243 .102915-0.932836$ $\begin{array}{llll}4.066970 & 0.940629 & -1.296624\end{array}$ $3.7300491 .766553 \quad 0.974649$ $3.5731513 .490806-2.198341$ $2.5555694 .000114-0.133436$ $4.407408 \quad 1.302932-2.582372$ $4.125943-0.364771-0.940495$ $4.2068952 .591712-3.060549$ $4.9520030 .365421 \quad-3.373374$ $3.387204 \quad 4.519039-2.491095$ $\begin{array}{llll}4.536929 & 2.863990 & -4.053801\end{array}$

$2.169136-0.5354440 .261026$ $3.569059-0.685921-1.060253$ $1.020306-1.902962-0.458253$ $-0.436119-0.8481841 .818397$ $-2.1103241 .3927011 .144945$ $4.908354-0.517213-0.585994$ $\begin{array}{llll}5.612248 & 0.679945 & -0.786865\end{array}$ $\begin{array}{llll}6.909763 & 0.775132 & -0.275876\end{array}$ $\begin{array}{llll}7.471010 & 1.694537 & -0.421404\end{array}$ $7.489098-0.2796220 .414674$ $8.499716-0.1876910 .800467$ $6.771634-1.4544380 .613008$ $7.229999-2.2756111 .156384$ $\begin{array}{llll}5.471627 & -1.592917 & 0.130449\end{array}$ $5.0094821 .866902-1.519007$ $4.0232011 .572440-1.896330$ $5.873284 \quad 2.299855-2.711077$ $6.0923961 .464015-3.381960$ $6.830916 \quad 2.708404-2.371113$ $\begin{array}{llll}5.365487 & 3.081423 & -3.285124\end{array}$ $\begin{array}{llll}4.816107 & 3.049082 & -0.560786\end{array}$ $\begin{array}{llll}4.194620 & 2.766482 & 0.294708\end{array}$ $\begin{array}{llll}4.342611 & 3.889970 & -1.079886\end{array}$ $5.7810513 .393505-0.172908$ $4.714913-2.8981660 .317965$ $3.654099-2.6967870 .159618$ $5.147926-3.923739-0.736058$ $4.972334-3.549236-1.750642$ $4.587015-4.857223-0.617113$ $6.215294-4.152030-0.639327$ $4.852676-3.4608991 .733063$ $\begin{array}{lll}4.546974 & -2.7147852 .469628\end{array}$ $5.877356-3.7815361 .949379$ $4.211130-4.3424631 .843655$ $3.286140-1.095829-2.291424$ $2.037749-1.627365-2.644136$ $1.897974-1.860943-3.691986$ $1.065787-2.152583-1.774270$ $0.122010-3.139254-2.428682$ $-0.769082-3.359006-1.848135$ $0.661001-4.069116-2.637999$ $-0.206704-2.714983-3.378640$ $4.349820-1.021150-3.356410$ 
$-5.010698-4.342506-1.043502$ $-6.876175-2.658346-2.160739$ $\begin{array}{lll}1.370626 & 5.516430 & 0.193480\end{array}$

TS5b

$\mathrm{Ga}$

$\mathrm{N}$

$\mathrm{N}$

$\mathrm{N}$

C

C

$\mathrm{C}$

$\mathrm{C}$

C

C

$\mathrm{H}$

$\mathrm{C}$

$\mathrm{H}$

$\mathrm{C}$

H

$\mathrm{H}$

C

H

$\mathrm{C}$ $\begin{array}{lll}-2.116864 & 0.371585 & 0.278153\end{array}$ $-3.5260920 .449431-1.044209$ $-1.0341241 .783929-0.464374$ $\begin{array}{llll}0.506277 & 0.821582 & 1.800751\end{array}$ $2.295128-1.3375681 .115973$ $-4.8567830 .226204-0.568058$ $-5.505045-1.004772-0.751889$ $-6.797625-1.151497-0.240452$ $-7.315859-2.097812-0.372616$ $-7.425852-0.1143050 .433691$ $-8.431850-0.2467010 .819902$
-6.763139 $-6.7631391 .0948320 .615355$ $-7.2593211 .9021341 .146214$ $\begin{array}{llll}-5.469816 & 1.285338 & 0.132348\end{array}$ $-4.848777-2.173636-1.466948$ $-3.875496-1.841555-1.846355$ $-5.690834-2.658847-2.654609$ $-5.946837-1.841948-3.335704$ $-6.629588-3.106096-2.310512$ $-5.147639-3.423523-3.219022$ $-4.605512-3.334968-0.494676$ $-3.998788-3.0172840 .358731$ $-4.091484-4.158069-1.003191$ $-5.555659-3.718548-0.106600$ $\begin{array}{llll}-4.772805 & 2.625863 & 0.302829\end{array}$ $\begin{array}{lll}-3.703822 & 2.469589 & 0.148671\end{array}$ $-5.2505753 .616684-0.765002$ $-5.0574413 .236790-1.774422$ $-4.7321394 .575898-0.658540$ $-6.3272483 .798198-0.671745$ $-4.9368943 .2003401 .710383$ $-4.5940202 .4797452 .455919$ $-5.9757653 .4736591 .924192$ $-4.3385504 .1132701 .808159$ $-3.2639040 .853591-2.281484$ $-2.0402431 .433146-2.644461$ $-1.9115211 .657806-3.695814$ $-1.0898562 .009194-1.782287$ $-0.1793113 .014646-2.458355$ $0.6844053 .310652-1.869892$ $-0.7574193 .906596-2.720400$ $0.1892112 .569974-3.385119$ $-4.3251180 .719065-3.343293$ $-4.378195-0.325003-3.671704$ $-4.0937681 .341367-4.208081$ $\begin{array}{lll}-5.309860 & 0.990528 & -2.953623\end{array}$ $\begin{array}{llll}0.909991 & 3.161587 & 2.525312\end{array}$ $\begin{array}{llll}1.847958 & 3.227684 & 1.955192\end{array}$ $\begin{array}{llll}0.273307 & 4.548059 & 2.638690\end{array}$ -0.624443 4.4808053 .268641 $0.962360 \quad 5.238701 \quad 3.134998$ $-0.1131745 .0782051 .255947$ $-0.6339516 .0369701 .345876$ $\begin{array}{llll}0.797963 & 5.257997 & 0.670902\end{array}$ $\begin{array}{lll}-0.989707 & 4.072217 & 0.505757\end{array}$ $-1.2338874 .448195-0.492953$ $-1.9430053 .9197121 .033025$ $\begin{array}{lll}-0.283076 & 2.714268 & 0.386596\end{array}$ $\begin{array}{llll}0.697346 & 2.872859 & -0.059840\end{array}$ $-0.0413852 .1876891 .809923$ $\begin{array}{lll}-1.005665 & 2.177588 & 2.324751\end{array}$ $\begin{array}{llll}0.273836 & 0.026427 & 2.855190\end{array}$ $0.812859-1.2605323 .002902$ $0.453356-1.8246313 .852956$ $1.752293-1.9012232 .195835$ $2.200586-3.2837552 .594138$ $3.271253-3.2893612 .822252$ $2.048937-3.9818051 .763791$ $1.642503-3.6383933 .461106$ $-0.6344250 .496073 \quad 3.968565$ $-1.6498600 .6429543 .589391$ $-0.2844001 .4406824 .393906$ $-0.662828-0.2497044 .762353$ $3.489091-1.8952540 .538982$ $3.403211-2.877736-0.457388$ $4.588488-3.325526-1.038725$ $4.549002-4.078451-1.820430$ 
$4.718411-1.3401960 .935926$

$2.058611-3.357223-0.971353$

$1.300572-3.091648-0.226803$

$2.012928-4.875822-1.165146$

$2.284980-5.409720-0.247767$

$2.698328-5.198610-1.955518$

$1.008284-5.185157-1.467149$

$1.698351-2.634724-2.280309$

$0.749292-3.009233-2.672999$

$2.477127-2.810728-3.030637$

$1.618501-1.550388-2.152554$

$4.832071-0.2714862 .016099$

$3.838090-0.0610972 .429761$

5.4077241 .0383811 .461312

$\begin{array}{llll}4.829203 & 1.413662 & 0.611886\end{array}$

$\begin{array}{llll}6.438386 & 0.895153 & 1.118450\end{array}$

$\begin{array}{llll}5.416178 & 1.807135 & 2.241339\end{array}$

$5.686238-0.7725773 .188834$

$5.278697-1.6952403 .614167$

$5.726942-0.0178223 .980756$

$6.713011-0.9763722 .867580$

1.1693842 .7615773 .511387

$-1.598643-1.5321090 .385044$

$-1.627253-2.2894621 .543281$

$-1.385530-2.229115-0.794399$

$-1.390047-3.6602251 .541463$

$-1.230592-3.607520-0.829977$

$-1.197440-4.3378720 .348897$

-1.049341 -5.411270 0.335019

$\begin{array}{llll}-2.785528 & 0.958028 & 1.839744\end{array}$

$-1.946862-1.7259412 .718090$

$-1.364515-4.3204742 .703808$

$-1.112965-4.236266-2.006767$

$-1.376299-1.557032-1.961291$

$\begin{array}{llll}1.865830 & 0.427239 & 0.434306\end{array}$

$3.0140621 .412946-1.267089$

$\begin{array}{llll}4.018821 & 0.674920 & -1.947543\end{array}$

$3.3286612 .790763-1.174601$

$\begin{array}{llll}1.658510 & 1.037080 & -1.751138\end{array}$

$5.1854421 .231618-2.419364$

$3.851159-0.655901-2.077345$

$4.4954453 .344742-1.665878$

$2.5352413 .607014-0.419125$

$\begin{array}{llll}5.458269 & 2.592997 & -2.319399\end{array}$

$\begin{array}{llll}6.073374 & 0.421056 & -3.012496\end{array}$

$\begin{array}{llll}4.692811 & 4.657343 & -1.470649\end{array}$

$6.3559653 .033383-2.728850$

TS5c

$\begin{array}{lll}-2.061415 & 0.183036 & 0.240893\end{array}$ $\begin{array}{lll}-3.562171 & 0.223963 & -0.967047\end{array}$ $-1.1112381 .671085-0.548126$ $\begin{array}{lll}0.601871 & 0.752545 & 1.610800\end{array}$ $2.453386-1.2964610 .812419$ $-4.868463-0.000391-0.430137$ $-5.482784-1.257624-0.527107$ $-6.780276-1.399590-0.030450$ $-7.272519-2.366443-0.094990$ $-7.444263-0.3280110 .552216$ $-8.456449-0.4530430 .924620$ $\begin{array}{llll}-6.803260 & 0.899730 & 0.676828\end{array}$ $-7.3196011 .7274541 .155263$ $\begin{array}{llll}-5.503984 & 1.083613 & 0.204516\end{array}$ $-4.732455-2.463726-1.061338$ $-3.800251-2.110600-1.516809$ $-5.515468-3.235095-2.128646$ $-5.806158-2.593210-2.966160$ $-6.429302-3.672540-1.712881$ $-4.907537-4.056151-2.522171$ $-4.373183-3.3925690 .107728$ $-3.910417-2.8373690 .928977$ $-3.681703-4.177660-0.217820$ $-5.276224-3.8722720 .502717$ $\begin{array}{llll}-4.8053642 .424816 & 0.362727\end{array}$ $\begin{array}{lll}-3.7424082 .270510 & 0.166708\end{array}$ $\begin{array}{lll}-5.323329 & 3.443086 & -0.658521\end{array}$ $-5.1597293 .096320-1.684614$ $-4.8066934 .401851-0.539649$

$-6.3971923 .616771-0.525344$ $-4.9171222 .9614691 .791341$ $-4.5152832 .2342382 .500446$ $-5.9529853 .1924752 .062135$ $-4.3443373 .8913091 .883989$ $-3.4006150 .640488-2.220667$ $-2.2411301 .287530-2.660364$ $-2.1953391 .529379-3.714542$ $-1.2810691 .926410-1.849104$ $-0.5249773 .035132-2.550526$ $0.363723 \quad 3.376374-2.027645$ $-1.2025313 .880044-2.712257$ $-0.2065222 .666716-3.527395$ $-4.5388720 .466361-3.193560$ $-4.810441-0.591511-3.269110$ $-4.2684030 .838531-4.181825$ $-5.4282580 .998745-2.841536$ $\begin{array}{llll}0.952065 & 3.097741 & 2.336559\end{array}$ $\begin{array}{llll}1.843534 & 3.214730 & 1.704974\end{array}$ $0.260627 \quad 4.4501942 .521580$ $-0.5794704 .3293473 .219574$ 0.9528155 .1672532 .974103 $-0.2616414 .9813161 .184391$ $-0.8254655 .9074581 .336034$ $\begin{array}{lll}0.589630 & 5.222146 & 0.535313\end{array}$ $-1.1390963 .9416290 .481695$ $-1.4840754 .323975-0.483880$ $-2.0360543 .7235121 .079713$ $\begin{array}{lll}-0.365830 & 2.631347 & 0.275826\end{array}$ $\begin{array}{llll}0.567573 & 2.854912 & -0.234519\end{array}$ $-0.0014862 .0931581 .669390$

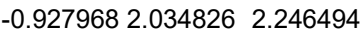
$0.471355-0.0493462 .674877$ $1.070637-1.3141982 .776985$ $0.783009-1.9043553 .636828$ $1.996352-1.8958841 .915863$ $2.547809-3.2486782 .286830$ $3.599543-3.1686422 .581695$ $2.507281-3.9217961 .424411$ $1.975273-3.6853973 .105776$ $\begin{array}{lll}-0.385725 & 0.371402 & 3.846809\end{array}$ $\begin{array}{ll}-1.4306760 .453255 & 3.530954\end{array}$ $-0.0703651 .3374704 .249422$ $-0.316819-0.3717594 .640636$ $3.686708-1.7635430 .235915$ $3.681931-2.688811-0.816922$ $4.905914-3.032918-1.389711$ $4.928501-3.737245-2.216641$ $6.092230-2.467266-0.938589$ $7.034077-2.736322-1.407196$ $6.075269-1.5608440 .114751$ $7.007077-1.1234700 .463440$ $4.875894-1.1985770 .729923$ $2.384695-3.272684-1.346047$ $1.604222-3.059006-0.609757$ $2.474904-4.795033-1.499823$ $2.811822-5.278086-0.576212$ $3.170487-5.076358-2.297421$ $1.495404-5.206881-1.765186$ $1.960228-2.630351-2.677075$ $1.086579-3.149854-3.084192$ $2.771578-2.704176-3.409544$ $1.712146-1.569536-2.577542$ $4.883672-0.2111871 .890607$ $3.879608-0.1635822 .327458$ $5.243414 \quad 1.201651 \quad 1.418619$ $\begin{array}{llll}4.537960 & 1.567047 & 0.666476\end{array}$ $\begin{array}{llll}6.237412 & 1.222678 & 0.958849\end{array}$ $\begin{array}{llll}5.239700 & 1.898334 & 2.263889\end{array}$ $5.834768-0.6656883 .004600$ $5.599371-1.6787113 .346402$ $\begin{array}{llll}5.762425 & 0.011142 & 3.861775\end{array}$ $6.876149-0.6597742 .666307$ $\begin{array}{llll}1.295688 & 2.695728 & 3.295716\end{array}$ $-1.370662-1.6640930 .264311$ $-1.291798-2.4585291 .391978$ $-1.133710-2.321718-0.937740$ $-0.922371-3.7997911 .340343$ $-0.849008-3.672387-1.052321$ $-0.713807-4.4173070 .120730$ $-0.456033-5.4708350 .099637$ $-2.6714100 .7274141 .842793$ 
$-1.645946-1.9675802 .590210$ $-0.793741-4.4823242 .486517$ $-1.225267-1.587087-2.069270$ $\begin{array}{llll}1.751778 & 0.352269 & 0.058883\end{array}$ $\begin{array}{llll}2.859725 & 1.528762 & -1.434102\end{array}$ $\begin{array}{llll}3.973845 & 0.827513 & -1.998496\end{array}$ $\begin{array}{llll}3.230588 & 2.873911 & -1.148017\end{array}$ $\begin{array}{llll}1.646798 & 1.386286 & -2.318600\end{array}$ $\begin{array}{llll}5.245600 & 1.311882 & -2.152517\end{array}$ $3.712113-0.446039-2.369357$ $\begin{array}{llll}4.501985 & 3.392581 & -1.321361\end{array}$ $\begin{array}{llll}2.305158 & 3.718300 & -0.602083\end{array}$ $\begin{array}{llll}5.549087 & 2.638974 & -1.819859\end{array}$ $\begin{array}{llll}4.691918 & 4.677278 & -0.975436\end{array}$ $\begin{array}{llll}5.992735 & 0.641683 & -2.564577\end{array}$ $6.5299573 .074456-1.952038$ $-0.714209-4.121842-2.029242$

\section{TS6a}

Ga

$\mathrm{N}$

N

C

C

$\mathrm{H}$

C

C

C

C

$\mathrm{H}$

$\mathrm{H}$

H

C

C

C

$\mathrm{H}$

$\mathrm{H}$

C

$\mathrm{H}$

C

$\mathrm{H}$

$\mathrm{H}$

C

$\mathrm{H}$

C

C

$\mathrm{H}$

$\mathrm{H}$

$\mathrm{C}$

$\mathrm{H}$

C

$\mathrm{H}$

$\mathrm{H}$

C

$\mathrm{H}$

$\mathrm{H}$

C

$\mathrm{H}$

$\mathrm{H}$
$-0.159364-0.361376-0.037770$ $-1.790414-1.2084370 .624540$ $0.982454-1.6991450 .809829$ $-1.865126-2.2944461 .393992$ $0.595020-2.7278111 .557746$ $-0.748421-3.0167251 .830278$ $-0.942439-3.8801192 .452373$ $-2.982417-0.5063630 .237127$ $-3.577121-0.802841-0.998289$ $-3.4415630 .5388161 .057541$ $-4.680875-0.041141-1.388979$ $-4.5430141 .2740960 .622954$ $-5.1620470 .985705-0.588613$ $-5.159657-0.249307-2.342176$ $-4.9151502 .0931451 .231012$ $\begin{array}{lll}-6.015299 & 1.572747 & -0.913622\end{array}$ $2.374292-1.4445440 .563540$ $3.026762-0.4762651 .344418$ $2.994185-2.056775-0.537764$ $4.348187-0.1617311 .023689$ $4.321169-1.722388-0.808126$ $4.995735-0.785864-0.034811$ $\begin{array}{llll}4.873812 & 0.591384 & 1.604572\end{array}$ $4.823345-2.178915-1.656854$ $6.022707-0.525634-0.270915$ $-3.050732-1.895587-1.916236$ $-2.181853-2.370190-1.444428$ $\begin{array}{llll}-2.717776 & 0.904996 & 2.345710\end{array}$ $-2.1473170 .0296732 .679414$ $\begin{array}{llll}2.327936 & 0.248839 & 2.486476\end{array}$ $1.355731-0.2266482 .666626$ $2.236248-2.988231-1.471350$ $1.253441-3.201372-1.034504$ $1.652427-3.6271412 .143262$ $2.231405-4.0997681 .343203$ $2.359739-3.0406992 .738464$ $1.208591-4.4017342 .768905$ $-3.227659-2.7613971 .838810$ $-3.876913-2.9278780 .974448$ -3.156736 -3.680727 2.420345 $-3.708627-1.9882232 .448187$ $\begin{array}{llll}2.072606 & 1.720884 & 2.130875\end{array}$ $\begin{array}{llll}3.011162 & 2.230796 & 1.885178\end{array}$ $\begin{array}{lll}1.413895 & 1.825194 & 1.261575\end{array}$ $\begin{array}{llll}1.607513 & 2.243327 & 2.973654\end{array}$ $\begin{array}{llll}3.126463 & 0.146380 & 3.791921\end{array}$ $3.338127-0.8958964 .050916$ $\begin{array}{llll}4.083169 & 0.673106 & 3.714841\end{array}$ $\begin{array}{llll}2.565926 & 0.598929 & 4.615832\end{array}$ $1.998457-2.304563-2.826897$ $1.474076-1.350014-2.723343$ $2.954359-2.102398-3.323024$ $1.409202-2.957365-3.480055$ $2.959210-4.325782-1.666657$ $3.155066-4.827639-0.713414$ $2.355481-4.994674-2.287964$ $-1.7071182 .0373642 .105357$ $-0.9043111 .7172591 .432040$ -2.185727 2.9036261 .636927 -1.248589 2.349437 3.049660 -3.681674 1.282705 3.474592 $\begin{array}{lll}-4.177721 & 2.238320 & 3.276931\end{array}$ $3.920374-4.185519-2.172156$ $\begin{array}{lll}-4.456681 & 0.521900 & 3.612262\end{array}$ $-3.1329821 .3907104 .415063$ $-4.103322-2.988851-2.142994$ $-4.987120-2.580608-2.644941$ $-3.696010-3.781987-2.778027$ $-4.434135-3.441355-1.202681$ $-2.591488-1.315180-3.260315$ $-3.443103-0.908624-3.817212$ $-1.859144-0.516383-3.125377$ $-2.139596-2.102790-3.872766$ $0.4249751 .426466-1.326396$ $\begin{array}{llll}1.785482 & 1.791677 & -1.408422\end{array}$ $-0.4787082 .485540-1.104798$ $-0.0492443 .752663-0.766926$ $2.2137113 .068944 \quad-1.106161$ $\begin{array}{llll}1.302719 & 4.079786 & -0.812314\end{array}$ $0.0129840 .449412-2.407696$ $-0.9447164 .685178-0.424859$ $-1.7723152 .149154-0.906064$ $\begin{array}{llll}2.696099 & 0.811387 & -1.565865\end{array}$ $3.5212463 .346218-1.109304$ $\begin{array}{llll}1.716073 & 5.334785 & -0.587558\end{array}$

TS6b

$\mathrm{Ga}$

N

$\mathrm{N}$

C

$-0.088731-0.241498-0.022315$ $-1.630859-1.2349820 .646614$ $1.177150-1.4591240 .830397$ $-1.600843-2.3088341 .435715$ $0.889950-2.5079751 .594950$ $-0.419996-2.9175911 .876611$ $-0.530594-3.7850672 .513303$ $-2.883955-0.6428810 .267576$ $-3.480790-1.020056-0.944925$ $-3.4044270 .388771 \quad 1.067465$ $-4.651137-0.361293-1.327807$ $\begin{array}{llll}-4.571063 & 1.021379 & 0.640629\end{array}$ $-5.1947480 .647594-0.544265$ $-5.132198-0.633712-2.263539$ $-4.9894701 .8298721 .232930$ $-6.0993601 .155370-0.863916$ $2.537760-1.0758900 .575551$ $3.087716-0.0218811 .323836$ $3.219517-1.657705-0.505591$ $\begin{array}{llll}4.372420 & 0.412067 & 0.993483\end{array}$ $4.507158-1.201248-0.786625$ $5.082638-0.177913-0.043816$ $4.8176891 .232759 \quad 1.549433$ $5.054811-1.631708-1.620694$ $\begin{array}{llll}6.079156 & 0.176033 & -0.288406\end{array}$ $-2.880349-2.083419-1.851552$ $-1.969474-2.475836-1.383641$ -2.687304 0.8599362 .324581 $\begin{array}{lll}-1.988682 & 0.075662 & 2.640652\end{array}$ $2.317964 \quad 0.6701472 .440595$ $\begin{array}{llll}1.390669 & 0.115120 & 2.630737\end{array}$ $2.559946-2.686406-1.411183$ $1.606256-2.989355-0.963334$ $2.028360-3.2953322 .189839$ $2.640424-3.7330481 .394494$ $2.685207-2.6344922 .764417$ $1.660602-4.0920242 .836772$ $-2.912227-2.8890201 .900024$ $-3.548413-3.1321571 .044180$ $-2.754658-3.7853952 .500065$ $-3.457845-2.1493272 .496290$ $\begin{array}{llll}1.936595 & 2.103211 & 2.041017\end{array}$ $2.8292772 .689235 \quad 1.794377$ $\begin{array}{lll}1.286319 & 2.126769 & 1.159545\end{array}$ $\begin{array}{llll}1.413293 & 2.602884 & 2.863307\end{array}$ $3.1122290 .675053 \quad 3.752763$ $3.408600-0.3373814 .044901$ $4.021966 \quad 1.277735 \quad 3.663934$ $2.509344 \quad 1.103566 \quad 4.559529$ $2.247108-2.064033-2.780961$ $1.623212-1.169322-2.697171$ $3.174890-1.770910-3.285017$ $1.731021-2.791727-3.416650$ $3.417770-3.945623-1.579215$ $4.355129-3.719221-2.097993$ $3.672638-4.398648-0.615459$ $2.883109-4.690477-2.176924$ $-1.8601312 .1246622 .043436$ 
$\begin{array}{lll}-4.568794 & 0.708484 & 0.628983\end{array}$

$\begin{array}{lll}-5.170365 & 0.188280 & -0.511027\end{array}$ $-5.008538-1.208106-2.131325$ $-5.0540391 .5167631 .168329$ $-6.1237710 .582576-0.848546$ $2.699022-0.6883720 .550167$ $\begin{array}{llll}3.141274 & 0.464431 & 1.221017\end{array}$ $3.426489-1.259418-0.506735$ $\begin{array}{llll}4.367570 & 1.011704 & 0.840774\end{array}$ $4.653818-0.686530-0.839591$ $\begin{array}{llll}5.124742 & 0.437166 & -0.171840\end{array}$ $\begin{array}{llll}4.729965 & 1.906100 & 1.340888\end{array}$ $5.234442-1.108126-1.655772$ $6.0759820 .877335-0.454530$ $-2.621919-2.400418-1.662011$ $-1.671903-2.669048-1.185064$ -2.657826 0.8508852 .284166 $-1.8755140 .1665802 .634530$ $\begin{array}{lll}2.318920 & 1.134318 & 2.313684\end{array}$ $\begin{array}{lll}1.447339 & 0.506972 & 2.538210\end{array}$ $2.870000-2.407896-1.334001$ $1.960100-2.779874-0.848884$ $2.448898-2.8545182 .295671$ $3.080151-3.2831451 .510362$ $3.053661-2.0999692 .808644$ $2.175177-3.6395313 .000969$ $-2.513816-2.9372532 .124687$ $-3.139159-3.2998831 .303822$ $-2.257703-3.7705542 .779310$ $-3.118199-2.2143062 .683673$ $\begin{array}{lll}1.806469 & 2.508699 & 1.859594\end{array}$ 2.6434403 .1758331 .624893 $\begin{array}{llll}1.186352 & 2.445120 & 0.958249\end{array}$ $\begin{array}{lll}1.212273 & 2.9740622 .653362\end{array}$ $3.1188681 .266756 \quad 3.615833$ $3.497842 \quad 0.296825 \quad 3.953275$ 3.9768401 .9349093 .487416 $2.4892951 .684477 \quad 4.407813$ $2.472363-1.911086-2.732734$ $1.750674-1.090282-2.690504$ $3.354355-1.546002-3.270974$ $2.036008-2.731152-3.313381$

$-1.9738552 .1740121 .904356$ $-1.1436882 .0138161 .207846$ $-2.6749382 .8537861 .408842$ $\begin{array}{lll}-1.5737102 .666031 & 2.797448\end{array}$ $\begin{array}{lll}-3.627659 & 1.068744 & 3.450757\end{array}$ $-4.3632951 .8464413 .221605$ $-4.1728920 .1517093 .696190$ $-3.0786411 .3920784 .340463$ $-3.469134-3.676041-1.756138$ $-4.420824-3.475985-2.260135$ $-2.940686-4.440700-2.334403$ $-3.696677-4.091919-0.769381$ $-2.302889-1.870819-3.066573$ $-3.225698-1.669457-3.622166$ $-1.721392-0.947227-3.025855$ $-1.732355-2.618381-3.628184$ $0.1983331 .561762-1.533799$ $\begin{array}{llll}1.458827 & 2.175890 & -1.716268\end{array}$ $-0.8830132 .451414-1.400539$ $-0.6875303 .810698-1.253471$ $1.6506483 .534318-1.620329$ $\begin{array}{llll}0.565125 & 4.391576 & -1.401124\end{array}$ $-0.0354400 .400946-2.464083$ $-1.7616474 .572148-0.983301$ $-2.0899431 .905652-1.129587$ $0.6741685 .466171-1.340671$ $2.5172381 .342671-1.843981$ $2.6595483 .918191-1.730269$ 


\section{References}

S1 Phanopoulos, A.; Leung, A. H.M.; Yow, S.; Palomas, D.; White, A. J. P.; Hellgardt, K.; Horton, A.; Crimmin, M. R. Binuclear $\beta$-diketiminate complexes of copper(I). Dalton Trans. 2017, 46, 2081-2090.

S2 Kysliak, O.; Görls, H.; Kretschmer, R. Salt metathesis as an alternative approach to access aluminium(I) and gallium(I) $\beta$-diketiminates. Dalton Trans. 2020, 49, 6377-6383. Jutzi, P.; Schebaum, L. O. A novel synthetic route to pentaalkylcyclopentadienylgallium(I) compounds. J. Organomet. Chem. 2002, 654, 176-179. COLLECT, Data Collection Software; Nonius B.V., Netherlands, 1998. „Processing of X-Ray Diffraction Data Collected in Oscillation Mode“: Z. Otwinowski, W. Minor in C. W. Carter, R. M. Sweet (eds.): Methods in Enzymology, Vol. 276, Macromolecular Crystallography, Part A, pp. 307-326, Academic Press 1997.

S6 SADABS 2.10, Bruker-AXS inc., 2002, Madison, WI, U.S.A

S7 Sheldrick, G. M. A short history of SHELX, Acta Cryst. 2008, A64, 112.

S8 Sheldrick, G. M. Crystal structure refinement with SHELXL, Acta Cryst. 2015, C71, 3.

S9 Dolomanov, O. V.; Bourhis, L. J.; Gildea, R. J.; Howard, J. A. K.; Puschmann, H. OLEX2: a complete structure solution, refinement and analysis program, J. Appl. Cryst. 2009, 42, 339.

S10 Gaussian 16, Revision C.01, M. J. Frisch, G. W. Trucks, H. B. Schlegel, G. E. Scuseria, M. A. Robb, J. R. Cheeseman, G. Scalmani, V. Barone, G. A. Petersson, H. Nakatsuji, X. Li, M. Caricato, A. V. Marenich, J. Bloino, B. G. Janesko, R. Gomperts, B. Mennucci, H. P. Hratchian, J. V. Ortiz, A. F. Izmaylov, J. L. Sonnenberg, D. Williams-Young, F. Ding, F. Lipparini, F. Egidi, J. Goings, B. Peng, A. Petrone, T. Henderson, D. Ranasinghe, V. G. Zakrzewski, J. Gao, N. Rega, G. Zheng, W. Liang, M. Hada, M. Ehara, K. Toyota, R. Fukuda, J. Hasegawa, M. Ishida, T. Nakajima, Y. Honda, O. Kitao, H. Nakai, T. Vreven, K. Throssell, J. A. Montgomery, Jr., J. E. Peralta, F. Ogliaro, M. J. Bearpark, J. J. Heyd, E. N. Brothers, K. N. Kudin, V. N. Staroverov, T. A. Keith, R. Kobayashi, J. Normand, K. Raghavachari, A. P. Rendell, J. C. Burant, S. S. lyengar, J. Tomasi, M. Cossi, J. M. Millam, M. Klene, C. Adamo, R. Cammi, J. W. Ochterski, R. L. Martin, K. Morokuma, O. Farkas, J. B. Foresman, D. J. Fox, Gaussian, Inc., Wallingford CT, 2016.

S11 Zhao, Y.; Truhlar, D. G. The M06 suite of density functionals for main group thermochemistry, thermochemical kinetics, noncovalent interactions, excited states, and transition elements: two new functionals and systematic testing of four M06-class functionals and 12 other functionals. Theor. Chem. Acc. 2008, 120, 215-241.

S12 a) Hehre, W. J.; Ditchfield, R.; Pople, J. A. Self-Consistent Molecular Orbital Methods. XII. Further Extensions of Gaussian-Type Basis Sets for Use in Molecular Orbital Studies of Organic Molecules. J. Chem. Phys. 1972, 56, 2257-2261; b) Francl, M. M.; Pietro, W. J.; Hehre, W. J.; Binkley, J. S.; Gordon, M. S.; DeFrees, D. J.; Pople, J. A. Self-consistent molecular orbital methods. XXIII. A polarization-type basis set for second-row elements. J. Chem. Phys. 1982, 77, 3654-3665 
S13 a) Neese, F. The ORCA program system. WIREs Comput. Mol. Sci. 2011, 2, 73-78; b) Neese, F. Software update: the ORCA program system, version 4.0" WIREs Comput. Mol. Sci. 2017, e1327.

S14 a) Riplinger, C.; Neese, F. An efficient and near linear scaling pair natural orbital based local coupled cluster method. J. Chem. Phys. 2013, 138, 34106; b) Riplinger, C.; Sandhoefer, B.; Hansen, A.; Neese, F. Natural triple excitations in local coupled cluster calculations with pair natural orbitals. J. Chem. Phys. 2013, 139, 134101.

S15 a) Dunning, T. H. Gaussian basis sets for use in correlated molecular calculations. I. The atoms boron through neon and hydrogen. J. Chem. Phys. 1989, 90, 1007-1023; b) Woon, D. E.; Dunning, T. H. Gaussian basis sets for use in correlated molecular calculations. III. The atoms aluminum through argon. J. Chem. Phys. 1993, 98, 1358-1371; c) Wilson, A. K.; Woon, D. E.; Peterson, K. A.; Dunning, T. H. Gaussian basis sets for use in correlated molecular calculations. IX. The atoms gallium through krypton. J. Chem. Phys. 1999, 110, 7667-7676.

S16 Weigend, F.; Köhn, A.; Hättig, C. Efficient use of the correlation consistent basis sets in resolution of the identity MP2 calculations. J. Chem. Phys. 2002, 116, 3175-3183.

S17 Neese, F.; Wennmohs, F.; Hansen, A.; Becker, U. Efficient, approximate and parallel HartreeFock and hybrid DFT calculations. A 'chain-of-spheres' algorithm for the Hartree-Fock exchange. Chem. Phys. 2009, 356, 98-109.

S18 Weigend, F. Accurate Coulomb-fitting basis sets for $\mathrm{H}$ to Rn. Phys. Chem. Chem. Phys, 2006, 8, 1057-1065.

S19 Lee, T. J.; Taylor, P. R. A diagnostic for determining the quality of single-reference electron correlation methods. Int. J. Quantum Chem. 1989, 36, 199-207.

S20 a) Henkelman, G.; Uberuaga, B. P.; Jónsson, H. A climbing image nudged elastic band method for finding saddle points and minimum energy paths. J. Chem. Phys. 2000, 113, 9901-9904; b) Henkelman, G.; Jónsson, H. Improved tangent estimate in the nudged elastic band method for finding minimum energy paths and saddle points. J. Chem. Phys. 2000, 113, 9978-9985; c) Smidstrup, S.; Pedersen, A.; Stokbro, K.; Jónsson, H. Improved initial guess for minimum energy path calculations. J. Chem. Phys. 2014, 140, 214106.

S21 a) CYLview, 1.0b; Legault, C. Y. Université de Sherbrooke, 2009 (http://www.cylview.org); b) Persistence of Vision Pty. Ltd. 2004 (http://www.povray.org).

S22 a) Kim, Y.; Cho, H.; Hwang, S. Density Functional Theoretical Study on the C-F and C-O Oxidative Addition Reaction at an Al Center. Bull. Korean Chem. Soc. 2017, 38, 282-284; b) Pitsch, C. E.; Wang, X. Aluminum(I) $\beta$-diketiminato complexes activate $\mathrm{C}\left(\mathrm{sp}^{2}\right)-\mathrm{F}$ and $\mathrm{C}\left(\mathrm{sp}^{3}\right)-\mathrm{F}$ bonds by different oxidative addition mechanisms: A DFT study. Chem. Commun. 2017, 53 , 8196-8198; c) Coates, G.; Rekhroukh, F.; Crimmin, M. R. Breaking Carbon-Fluorine Bonds with Main Group Nucleophiles. Synlett 2019, 30, 2233-2246; d) Zhang, X.; Li, P.; Wang, B.; Cao, Z. Mechanistic Features in $\mathrm{Al}(\mathrm{I})-$ Mediated Oxidative Addition of Aryl C-F Bonds: Insights From Density Functional Theory Calculations. Front. Chem. 2019, 7, 596. 